\title{
THE GENERATION AND DEVELOPMENT
}

OF A VISCOUS VORTEX RING

\author{
A THESIS \\ Presented to \\ The Faclilty of the Graduate Division \\ by \\ Kenton David Whitehead
}

\author{
In Partial Fulfillment \\ of the Requirements for the Degree \\ Doctor of Philosophy \\ in the School of Aerospace Engineering
}

Georgia Institute of Technology

June, 1967 


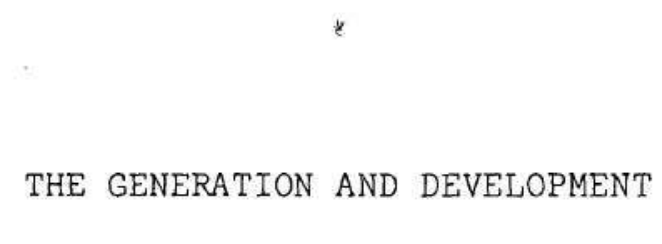

OF A VISCOUS VORTEX RING

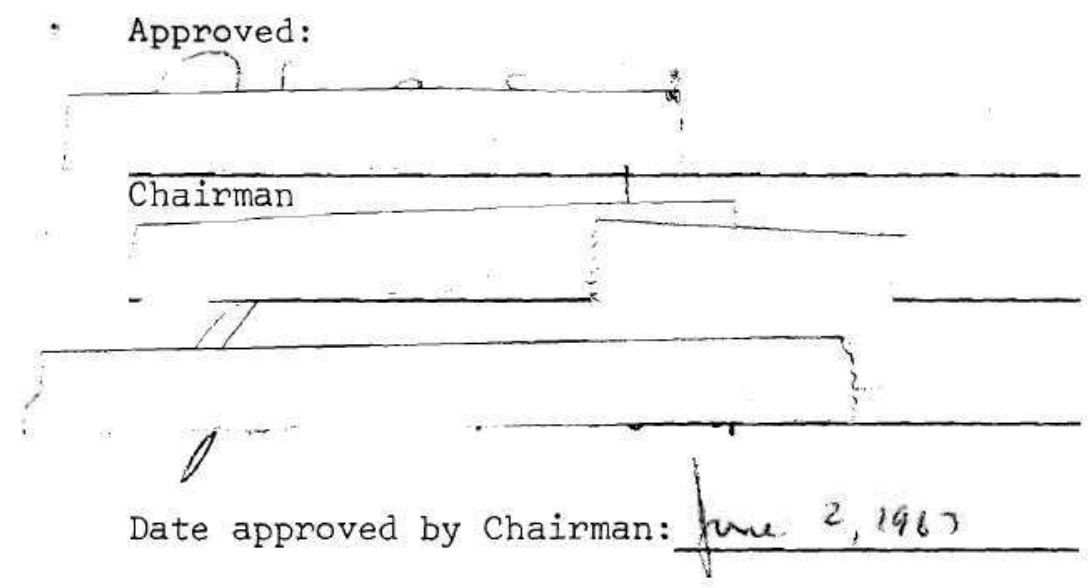

$-$ 
In presenting the dissertation as a partial fulfillment of the requirements for an advanced degree from the Georgia Institute of Technology, I agree that the Library of the Institute shall make it available for inspection and circulation in accordance with its regulations governing materials of this type. I agree that permission to copy from, or to publish from, this dissertation may be granted by the professor under whose direction it was written, or, in his absence, by the Dean of the Graduate Division when such copying or publication is solely for scholarly purposes and does not involve potential financial gain. It is understood that any copying from, or publication of, this dissertation which involves potential financial gain will not be allowed without written permission.

$3 / 17 / 65$

b 


\section{ACKNOWLEDGMENTS}

I wish to express my deepest appreciation to Dr. Robin B. Gray who suggested this thesis topic and provided the encouragement and wisdom necessary for the pursuit of both this research and a great portion of my graduate academic career. To Professor James E. Hubbartt and to Dr. James C. Wu I owe special thanks for their many hours of fruitful discussion during the investigation and the preparation of the manuscript.

The counsel of my fellow graduate students is also gratefully acknowledged in the light of many helpful suggestions by Drs. Richard G. Bradley, Don P. Giddens, Jerry A. Sills, and Joseph D. Stewart. Dr. Bradley is also the source of some preliminary experimental data. With respect to the many numerical computations of this study, I wish to thank Messrs. Pete Jensen and Payne Lenoir, the staff, and the machine operators of the Rich Electronic Computer Center for many hours of valuable service. To Mrs. Jimmie Bradley, Miss Mary Dale, and fellow student Mr. Robert Sigman for their careful study of the manuscript and to Mrs. Sue Bailey for her helpful suggestions, many thanks are given. I also express my thanks to Mrs. Betty R. Sims for her patience and skill in typing the final draft of this thesis.

The financial assistance of the National Defense Education Act, the Ford Foundation, the National Aeronautics and Space Administration, and the Georgia Institute of Technology are gratefully acknowledged. 
I owe a special debt of gratitude to Dr. Arnold L. Ducoffe, Director of the School of Aerospace Engineering, for his advice and encouragement during my stay at Georgia Tech. To my parents I owe thanks for my entire academic career.

My wife Betsy has provided both inspiration and encouragement throughout this research, and it is largely through her patience and understanding that this work has been completed. 


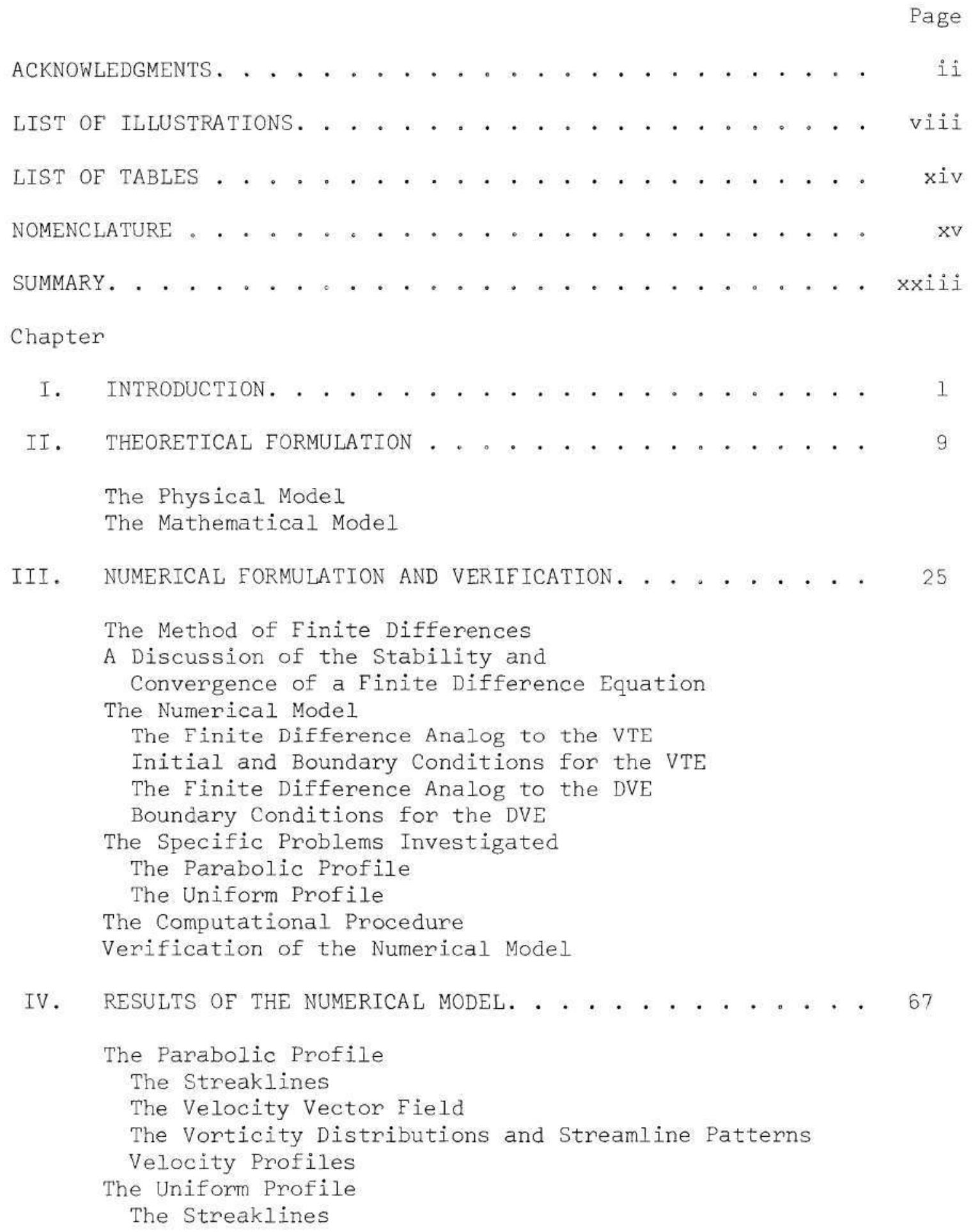


IV. RESULTS OF THE NUMERICAL MODEL (Continued)

The Uniform Profile (Continued)

The Velocity Vector Field

The Vorticity Distributions and Streamline Patterns

Velocity Profiles

Concluding Remarks

V. INVESTIGATION OF A SLOWLY DIFFUSING VORTEX RING . . . . . 147

Computation of the Axial Location of the Vortex Center

Computation of the Vortex Velocity

Computation of the Radial Location of the Vortex Center

The Numerical Results in the Moving Co-ordinate System

Calculation of the Circulation

Correlation of Existing Vortex Models

Lamb's Vortex Ring

Hill's Spherical Vortex

Concluding Remarks

VI. CONCLUSIONS AND RECOMMENDATIONS ............. 181

Conclusions

Recommendations

The Numerical Model

The Problem of the Viscous Vortex Ring

APPENDIX

A. ATTEMPTED SOLUTIONS OF THE SYSTEM COMPRISED OF THE

VORTICITY TRANSPORT EQUATION, THE DEFINITION OF

VORTICITY EQUATION, AND THE CONTINUITY EQUATION . . . . 189

Method I

Method II

Method III

Concluding Comments

B. DERIVATION OF THE PRESSURE EQUATION . . . . . . . . 196

The Derivation

Boundary Condition for Large $z$

Boundary Condition for Large $r$

Boundary Condition at $r=0$

Boundary Condition at $z=0$ 
B. DERIVATION OF THE PRESSURE EQUATION (Continued)

Suggestions for the Boundary Conditions

Evaluation of the Pressure at Large Distances

Evaluation of the Term $\partial \mathrm{p} / \partial r=0$

Evaluation of the Term $\partial v_{r} / \partial z$

Evaluation of the Term $\partial \omega / \partial r$

C. THE FINITE DIFFERENCE TECHNIQUE . . . . . . . ....

First Derivative--Central Difference Approximation

First Derivative--Forward Difference Approximation

First Derivative--Backward Difference Approximation

First Derivative--Central Difference Approximation

(Unequally Spaced Grid)

Second Derivative--Central Difference Approximation

D. STABIlity ANALYSIS OF THE VTE . . . . . . . . . .

Case I. Forward Time Difference--Explicit Formulation of the Simplified VTE

Case II. Central Time Difference--Explicit Formulation of the Simplified VTE

Case III. Central Time Difference--Explicit Formulation of the Simplified VTE (Time Average Technique)

Case IV. Forward Time Difference--Implicit Formulation of the Simplified VTE

Case V. Forward Time Difference--Implicit Formulation of the Unsimplified VTE

Conclusions

E. FINITE DIFFERENCE FORMS OF THE VORTICITY TRANSPORT

EQUATION. . . . . . . . . . . . . . . . . . . . .

The Simplified VTE

Case I. Forward Time Difference--Explicit Formulation of the Simplified VTE

Case II. Central Time Difference--Explicit Formulation of the Simplified VTE

Case III. Central Time Difference--Explicit Formulation of the Simplified VTE (Time Average Technique)

Case IV. Forward Time Difference--Implicit Formulation of the Simplified VTE

The Unsimplified VTE

Case V. Forward Time Difference--Implicit

Formulation of the Unsimplified VTE 
F. FINITE DIFFERENCE FORM OF THE DEFINITION OF VORTICITY EQUATION. . . . . . . . . . . . . 277

G. SOME ITERATIVE METHODS FOR THE NUMERICAL SOLUTION OF ELLIPTIC PARTIAL DIFFERENTIAL EQUATIONS. . . . . . . 282

Method I. Jacobi's Method

Method II. The Gauss-Seidel or Liebmann Method

Method III. The Method of Successive Over-Relaxation

Method IV. Refining the Mesh

Method V. The Combination Method

Method VI. The Alternating Direction Method

H. THE CONVERGENCE STUDY . . . . . . . . . . . . 294

The Parabolic Profile

The Uniform Profile

Concluding Remarks

I. A STEADY STATE SOLUTION . . . . . . . . . . . . . . 331

J. DEVELOPMENT OF LAMB'S VORTEX MODEL. . . . . . . . . . . . 350

K. HILL'S SPHERICAL VORTEX ............. 365

LITERATURE CITED . . . . . . . . . . . . . . . . . . 371

VITA . . . . . . . . . . . . . . ..... 375 
LIST OF ILLUSTRATIONS

Figure

Page

1. Formation of a Viscous Vortex Ring. . . . . . . . . . . 7

2. Geometry of the Physical Model. . . . . . . . . . . . 10

3. A Control Surface and a Portion

of the Finite Difference Grid . . . . . . . . . . 26

4. Mesh Spacing in the Transformed Plane .......... 33

5. Linear Impulsive Center Line Profile........... 50

6. Instantaneous Impulsive Center Line Profile ........ 51

7. Uniform Velocity Profile.............. 54

8. Schematic Diagram of the Computational Procedure. . . . 62

9. Streakline Pattern at $t=2$-- Parabolic Profile. .... 71

10. Streakline Pattern at $t=6$-- Parabolic Profile. . . . 72

11. Streakline Pattern at $t=10$-- Parabolic Profile . . . 73

12. Streakline Pattern at $t=14$-- Parabolic Profile . . . 74

13. Streakline Pattern at $t=18$-- Parabolic Profile . . . 75

14. Portion of Velocity Vector Field at $t=10--$

15. Portion of Streamline Pattern at $t=10$-- Parabolic Profile.......... 81

16. Vorticity Distribution and Streamline
Pattern at $t=4_{-- \text {Parabolic Profile . . . . . . . } 83}$

17. Vorticity Distribution and Streamline
Pattern at $t=8$-- Parabolic Profile . . . . . . . . . 84

18. Vorticity Distribution and Streamline

Pattern at $t=12$-- Parabolic Profile. . . . . . . 85

19. Vorticity Distribution and Streamline

Pattern at $t=16$-- Parabolic Profile. . . . . . . 86 
20. Vorticity Distribution and Streamline Pattern at $t=20$-- Parabolic Profile. . . . . . . .

21. Vorticity Distribution and Streamline Pattern at $t=24$-- Parabolic Profile. . . . . . . .

22. Vorticity Distribution and Streamline

Pattern at $t=28$-- Parabolic Profile. . . . . . . . .

23. Vorticity Distribution and Streamline Pattern at $t=32$-- Parabolic Profile. . . . . . . . . 90

24. Vorticity Distribution and Streamline Pattern at $\mathrm{t}=36--$ Parabolic Profile. . . . . . . . .

25. Vorticity Distribution and Streamline

Pattern at $t=40$-- Parabolic Profile. . . . . . . .

26. Vorticity Distribution and Streamline

Pattern at $t=44$-- Parabolic Profile. . . . . . . . . .

27. Vorticity Distribution and Streamline

Pattern at $t=48$-- Parabolic Profile. . . . . . . . . 94

28. Vorticity Distribution and Streamline

Pattern at $t=52$-- Parabolic Profile. . . . . . . . .

29. Vorticity Distribution and Streamline

Pattern at $t=56$-- Parabolic Profile. . . . . . . . 96

30. Axial Velocity Distribution near Vortex

Center at $t=40$-- Parabolic Profile . . . . . . . . 107

31. Axial Center Line Velocity

at $t=40$-- Parabolic Profile... . . . . . . . . 108

32. Axial Center Line Velocities at

Different Times -- Parabolic Profile. . . . . . . . . 110

33. Maximum Center Line Velocity as a

Function of Time -- Parabolic Profile . . . . . . . . . 112

34. Streamline Pattern about Vortex

Filament According to Ringleb [9] . . . . . . . . . . 113

35. Streakline Pattern at $t=2$-- Uniform Profile. . . . . 115

36. Streakline Pattern at $t=6$-- Uniform Profile. . . . . . 116 
37. Streakline Pattern at $t=10$-- Uniform Profile . . . . . . 117

38. Streakline Pattern at $t=14$-- Uniform Profile . . . . . 118

39. Portion of Velocity Vector Field at $t=8$-- Uniform Profile. . . . . . . . . . . . . 122

40. Portion of Streamline Pattern at $t=8$-- Uniform Profile.... . . . . . . . 123

41. Vorticity Distribution at $t=12$-- Uniform Profile . . . 125

42. Vorticity Distribution and Streamline Pattern at $t=4$-- Uniform Profile. . . . . . . . . . 129

43. Vorticity Distribution and Streamline Pattern at $t=8$-- Uniform Profile... . . . . . . 130

44. Vorticity Distribution and Streamline Pattern at $t=12$-- Uniform Profile. . . . . . . . . . 131

45. Vorticity Distribution and Streamline Pattern at $t=16$-- Uniform Profile. . . . . . . . . 132

46. Vorticity Distribution and Streamline Pattern at $t=20$-- Uniform Profile. . . . . . . . . 133

47. Vorticity Distribution and Streamline Pattern at $t=24$-- Uniform Profile. . . . . . . . . 134

48. Vorticity Distribution and Streamline Pattern at $t=28$-- Uniform Profile. . . . . . . . . . 135

49. Axial Velocity Distribution near Vortex Center at $t=28$-- Uniform Profile. . . . . . . . . . 141

50. Axial Velocity Distributions Near Vortex Center at Various Times -- Uniform Profile. . . . . . . . . 143

51. Axial Center Line Velocity at $t=28$-- Uniform Profile . 144

52. Illustration for Calculation of the Vortex Center . . . . 149

53. Axial Location of Vortex Center as Function of Time -- Parabolic Profile. . . . . . . . 150

54. Propagation Velocity of Vortex as Function of Time -- Parabolic Profile . . . . . . . . . 153 
55. Radial Location of Vortex Center as Function of Time -- Parabolic Profile. . . . . . . . . 157

56. Vorticity Distribution and Streamline Pattern at $t=40$-- Parabolic Profile Moving Co-ordinate System. . 158

57. Streamline Pattern of Hill's Spherical Vortex as Given by Ringleb [9]. . . . . . . . . 160

58. Portion of $\mathrm{z}, \mathrm{r}$ Plane Used in the Calculation of the Circulation. . . . . . . . . . . . 162

59. Circulation Along Streamline as Function of Area Enclosed by Streamline, Parabolic Profile -- Fixed Co-ordinate System. . . . . . . . . . . . . . 164

60. Circulation Along Streamline as Function of Area Enclosed by Streamline, Parabolic Profile -Moving Co-ordinate System . . . . . . . . . . . . 165

61. Postulated Comparison of the Circulations of Lamb's Vortex Ring and the Viscous Vortex Ring. . . . . . . 172

62. Propagation Velocity of Vortex as Function of Time. . . . 179

63. Proposed Geometry for Recommended Vortex Study. . . . . . 185

C-1. Mesh Spacing in the Transformed Plane . . . . . . . . 215

C-2. Portion of Grid of Unequally Spaced Mesh Points . . . . . 220

C-3. Illustration of Use of Five-Point

Formula Near a Boundary . . . . . . . . . . . . . 223

H-1. Vorticity Distribution at $t=2$-- Parabolic Profile. . . . 297

H-2. Vorticity Distribution at $t=6$-- Parabolic Profile. . . 298

H-3. Vorticity Distribution at $t=10$-- Parabolic Profile . . 299

H-4. Vorticity Distribution at $t=14$-- Parabolic Profile.. . 300

H-5. Vorticity Distribution at $t=18$-- Parabolic Profile . . . 301

H-6. Streamline Pattern at $t=2$-- Parabolic Profile. . . . . 302

H-7. Streamline Pattern at $t=6$-- Parabolic Profile. . . . 303 
Figure

Page

H-8. Streamline Pattern at $t=10$-- Parabolic Profile . . . . 304

H-9. Streamline Pattern at $t=14$-- Parabolic Profile . . . . 305

H-10. Streamline Pattern at $t=18$-- Parabolic Profile . . . . 306

H-ll. Vorticity at $z=1, r=1$-- Parabolic Profile. . . . . . . 307

H-12. Stream Function at $z=1, r=1$-- Parabolic Profile. . . . . . 308

H-13. Axial Velocity at $z=1, r=1$-- Parabolic Profile . . . . . 309

H-14. Radial Velocity at $z=1, r=1$-- Parabolic Profile. . . . . 310

H-15. Vorticity at $z=0, r=1$-- Parabolic Profile. . . . . . . . 311

H-16. Vorticity Distribution at $t=2$-- Uniform Profile. . . . 313

H-17. Vorticity Distribution at $t=6$-- Uniform Profile. . . . 314

H-18. Vorticity Distribution at $t=10$-- Uniform Profile . . . 315

H-19. Vorticity Distribution at $t=14$-- Uniform Profile . . . 316

H-20. Vorticity Distribution at $t=18$-- Uniform Profile . . . 317

H-21. Streamline Pattern at $t=2$-- Uniform Profile. . . . . 318

H-22. Streamline Pattern at $t=6$-- Uniform Profile. . . . . . 319

H-23. Streamline Pattern at $t=10$-- Uniform Profile . . . . . 320

H-24. Streamline Pattern at $t=14$-- Uniform Profile . . . . . 321

H-25. Streamline Pattern at $t=18$-- Uniform Profile . . . . . 322

H-26. Vorticity at $\mathrm{z}=1, \mathrm{r}=1$-- Uniform Profile. . . . . . . . 323

H-27. Stream Function at $z=1, r=1$-- Uniform Profile. . . . . . 324

H-28. Axial Velocity at $z=1, r=1$-- Uniform Profile.... . . 325

H-29. Radial Velocity at $z=1, r=1$-- Uniform Profile. . . . . . 326

H-30. Vorticity at $\mathrm{z}=0, \mathrm{r}=1$-- Uniform Profile. . . . . . . . 327

I-1. Center Line Profile for Steady State Solution . . . . . . . 332 
I-2. Physical Configuration for Axisymmetric

Flow from a Small Round Hole. . . . . . . . . . . . .

I-3. Physical Configuration for Axisymmetric

Flow from a Finite Round Hole . . . . . . . . . . . . . 338

I-4. Vorticity Distribution of the Boundary Layer Solution . . 343

I-5. Streamline Pattern of the Boundary Layer Solution . . . . 344

I-6. Streamline Pattern of the Numerical

Solution at $t=0, M=24 . . . . . . . .345$

I-7. Vorticity Distribution of the

Numerical Solution at $t=6, M=24$........ 346

I-8. Streamline Pattern of the Numerical

Solution at $t=6, M=24$. . . . . . . . . . 347

I-9. Streamline Pattern of the Numerical

Solution at $t=0, M=50$. . . . . . . . . . 349

J-1. Orientation of Vortex Core. . . . . . . . . . . . . 351

J-2. Orientation of Vortex Filaments in Vortex Core. . . . . . 355

K-1. Orientation of Hill's Spherical Vortex. . . . . . . . . . 367 
xiv

LIST OF TABLES

Table

Page

1. Summary of Equations, Initial Conditions, and General Boundary Conditions . . . . . . . . . . . . .

2. Summary of Equations, Initial Conditions, and Specific Boundary Conditions. . . . . . . . . . . 57

3. Values of the Stream Function for the Plotted Streamlines--Parabolic Profile Case . . . . . . . . . . . 97

4. Values of the Stream Function for the Plotted Streamlines--Uniform Profile Case . . . . . . . . . . 136

5. Core Size of Lamb's Vortex Ring Using Vorticity . . . . . 174

6. Core Size of Lamb's Vortex Ring Using Circulation . . . . . 175

H-1. Parameters Used in the Convergence Study of the Parabolic Profile. . . . . . . . . . . . . 295

H-2. Parameters Used in the Convergence Study of the Uniform Profile. . . . . . . . . . . . . 312 


\title{
NOMENCLATURE
}

\section{Roman Symbols}

\author{
a \\ $a_{0}$ \\ A \\ A \\ $\overline{\mathrm{A}}$ \\ b \\ B \\ $B$ \\ c \\ D \\ DVE \\ $E_{1}()$ \\ f \\ $f(r)$ \\ $F(\psi)$ \\ $F_{1}()$ \\ FF \\ g \\ transformation constant in the axial direction given \\ by Equation (25) \\ radius of Hill's spherical vortex \\ area or dummy function used in stability analysis \\ general square matrix \\ constant in derivation of Hill's spherical vortex \\ transformation constant in the radial direction given by \\ Equation (26) \\ dumny function used in stability analysis \\ general column matrix \\ constant in steady state solution of free jet \\ exact solution to a partial differential equation \\ definition of vorticity equation \\ complete elliptic integral of the second kind \\ arbitrary function \\ function which specifies the velocity profile in the \\ exit plane \\ function describing the vorticity in Hill's spherical \\ vortex \\ complete elliptic integral of the first kind \\ dummy function used in stability analysis \\ function in steady state solution of free jet
}




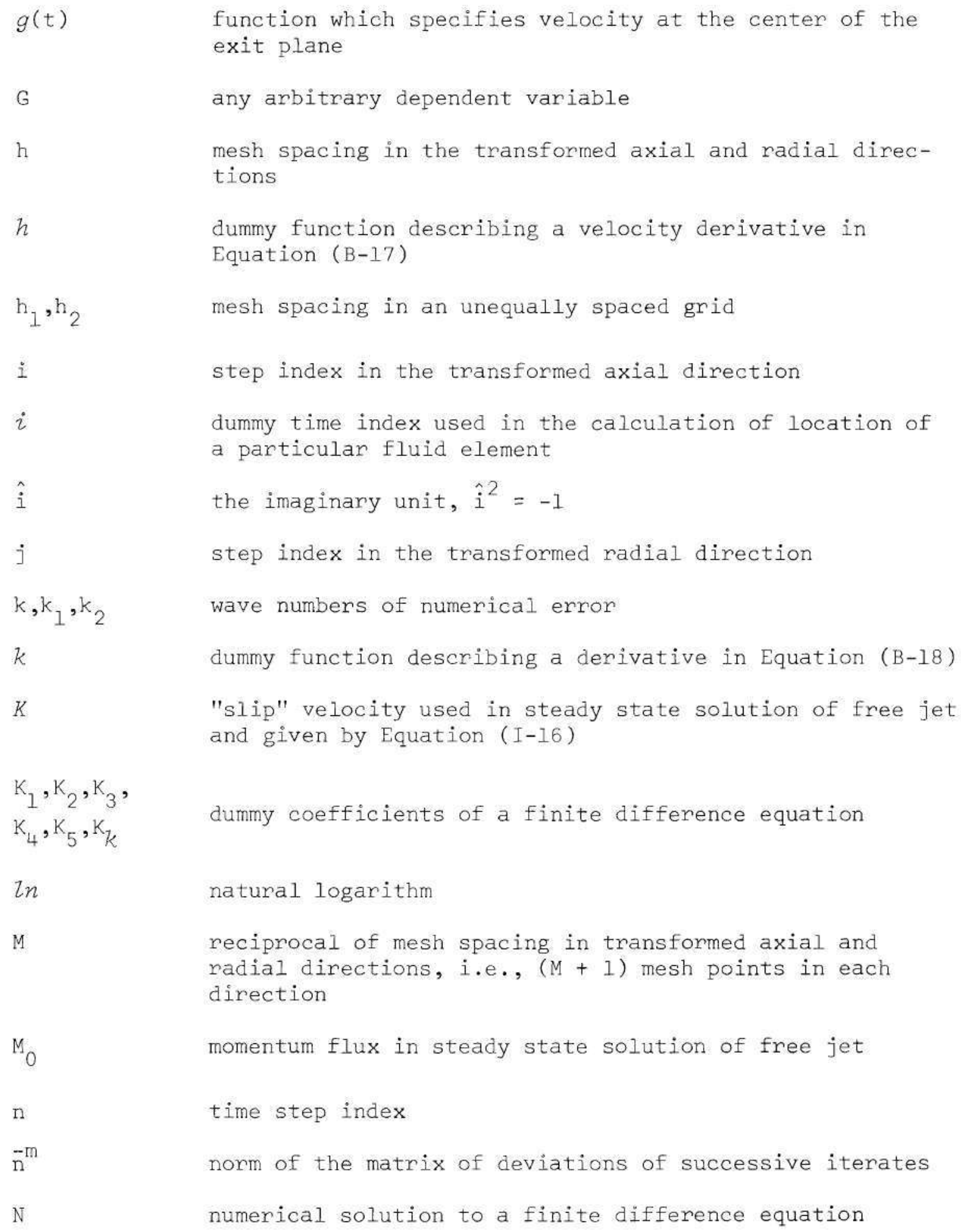

G

h

h

any arbitrary dependent variable

mesh spacing in the transformed axial and radial directions

dummy function describing a velocity derivative in Equation (B-17)

mesh spacing in an unequally spaced grid

step index in the transformed axial direction

dummy time index used in the calculation of location of a particular fluid element

the imaginary unit, $\hat{i}^{2}=-1$

step index in the transformed radial direction

wave numbers of numerical error

dummy function describing a derivative in Equation ( $B-18$ )

"slip" velocity used in steady state solution of free jet and given by Equation (I-16)

dummy coefficients of a finite difference equation

natural logarithm

reciprocal of mesh spacing in transformed axial and radial directions, i.e., $(M+1)$ mesh points in each direction

momentum flux in steady state solution of free jet

time step index

norm of the matrix of deviations of successive iterates

numerical solution to a finite difference equation 
Page missing from thesis 


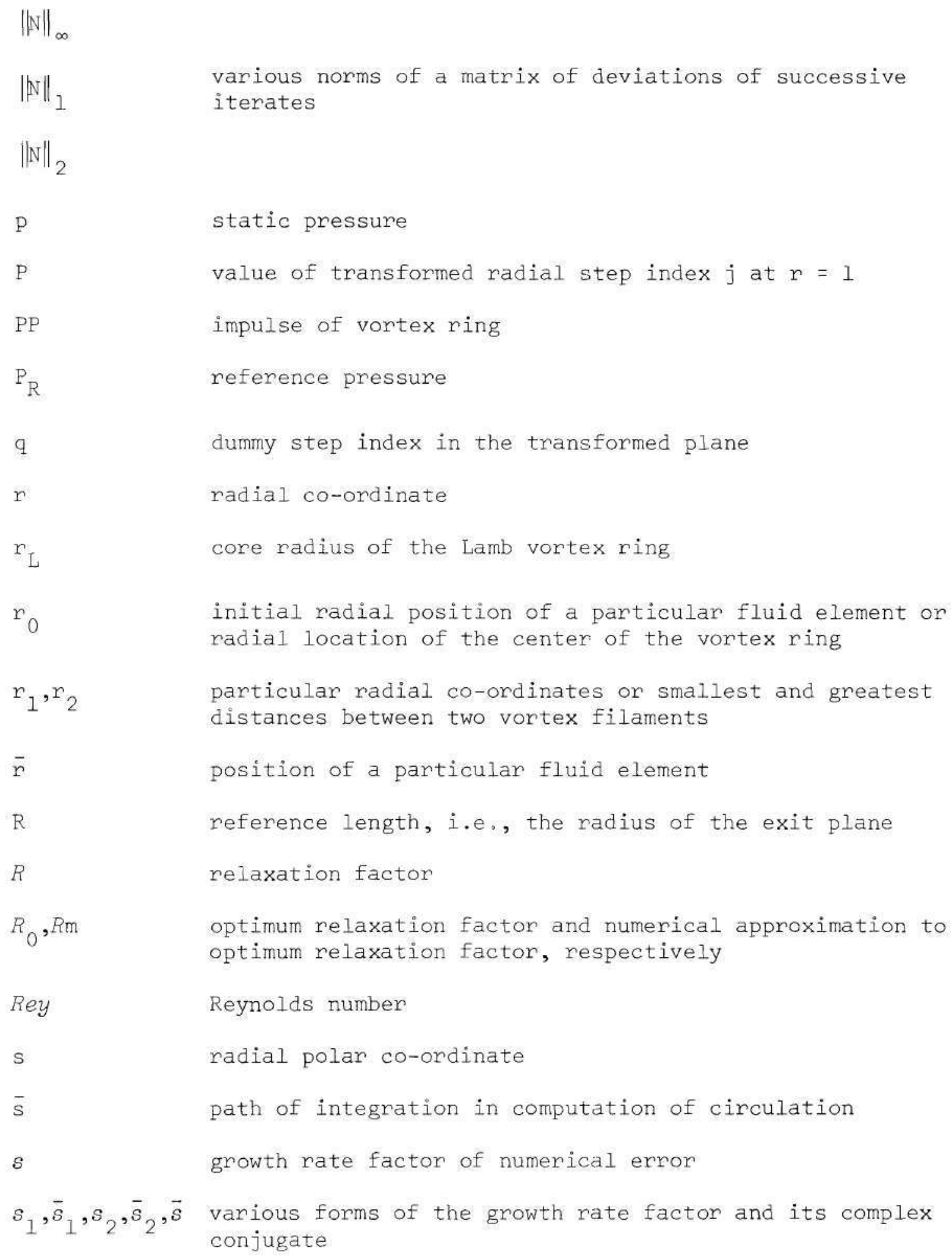




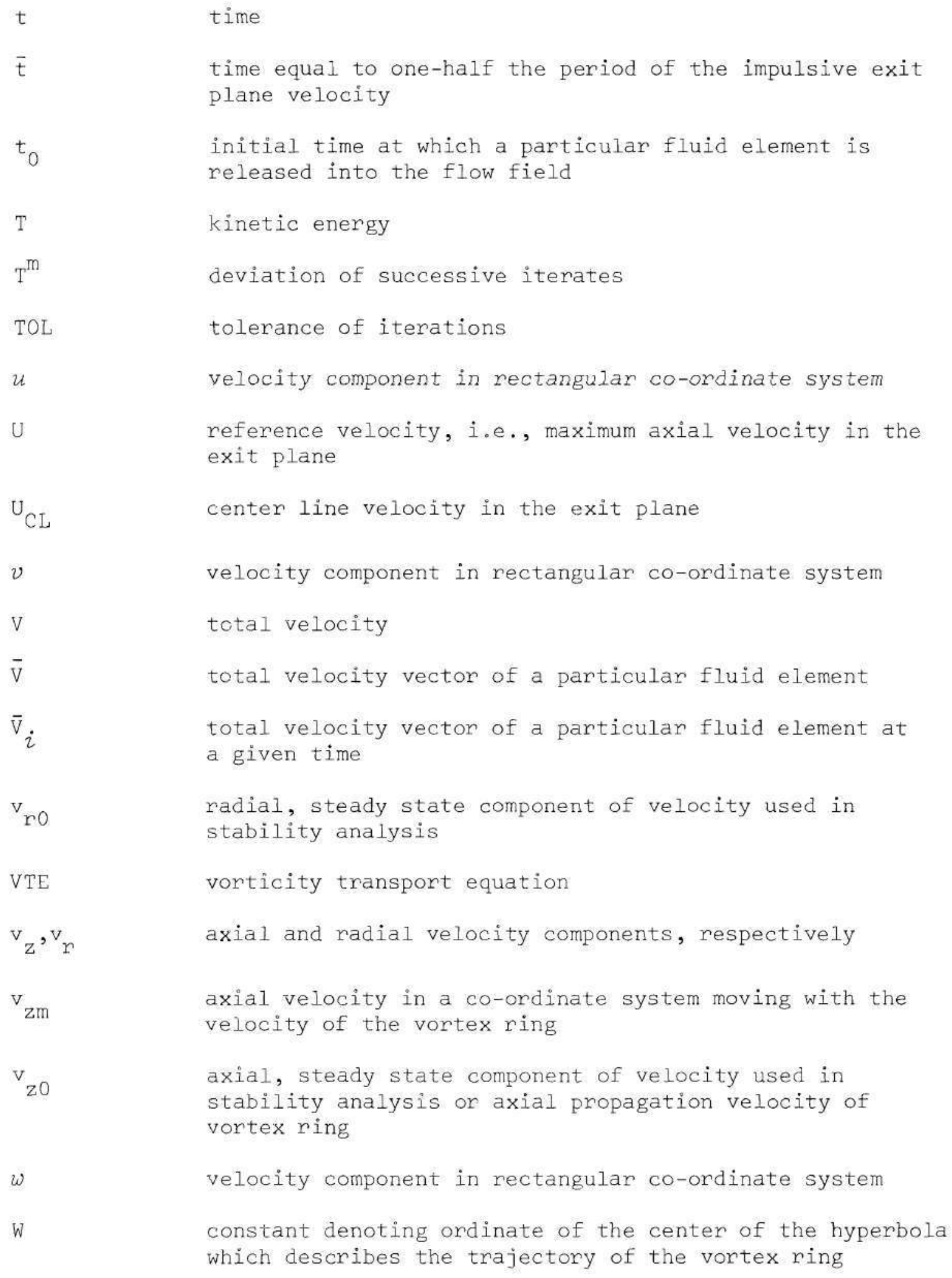




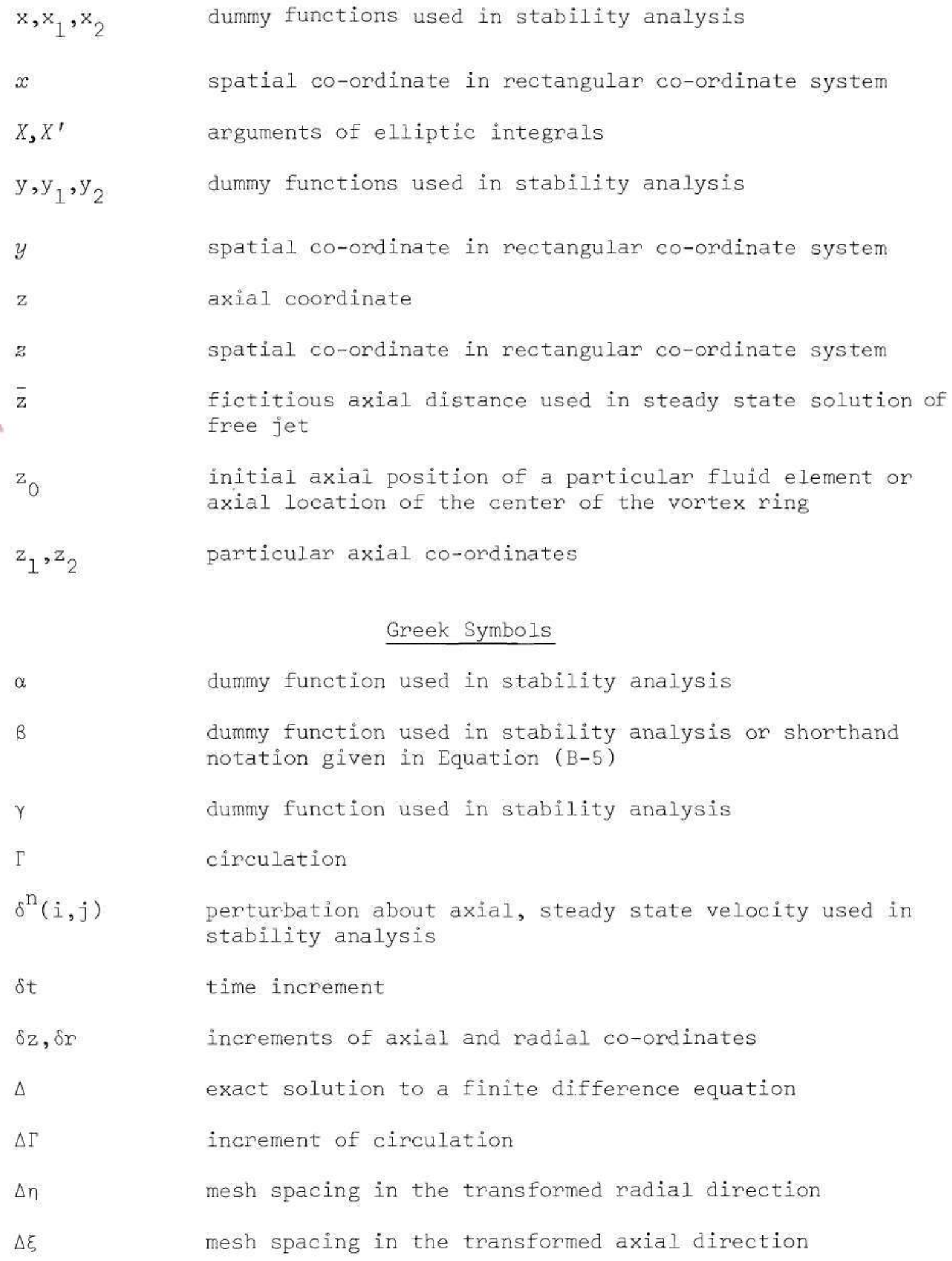




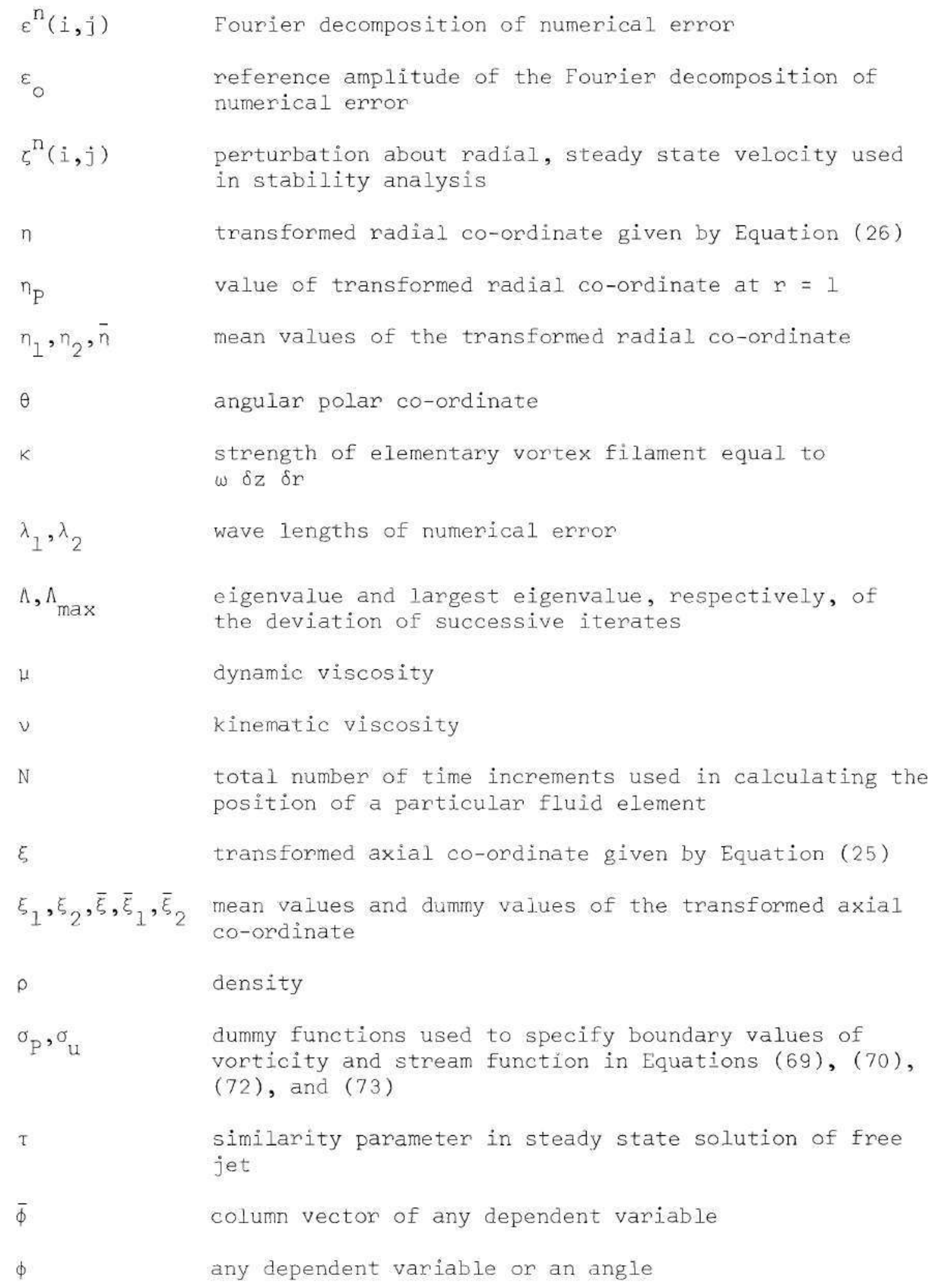




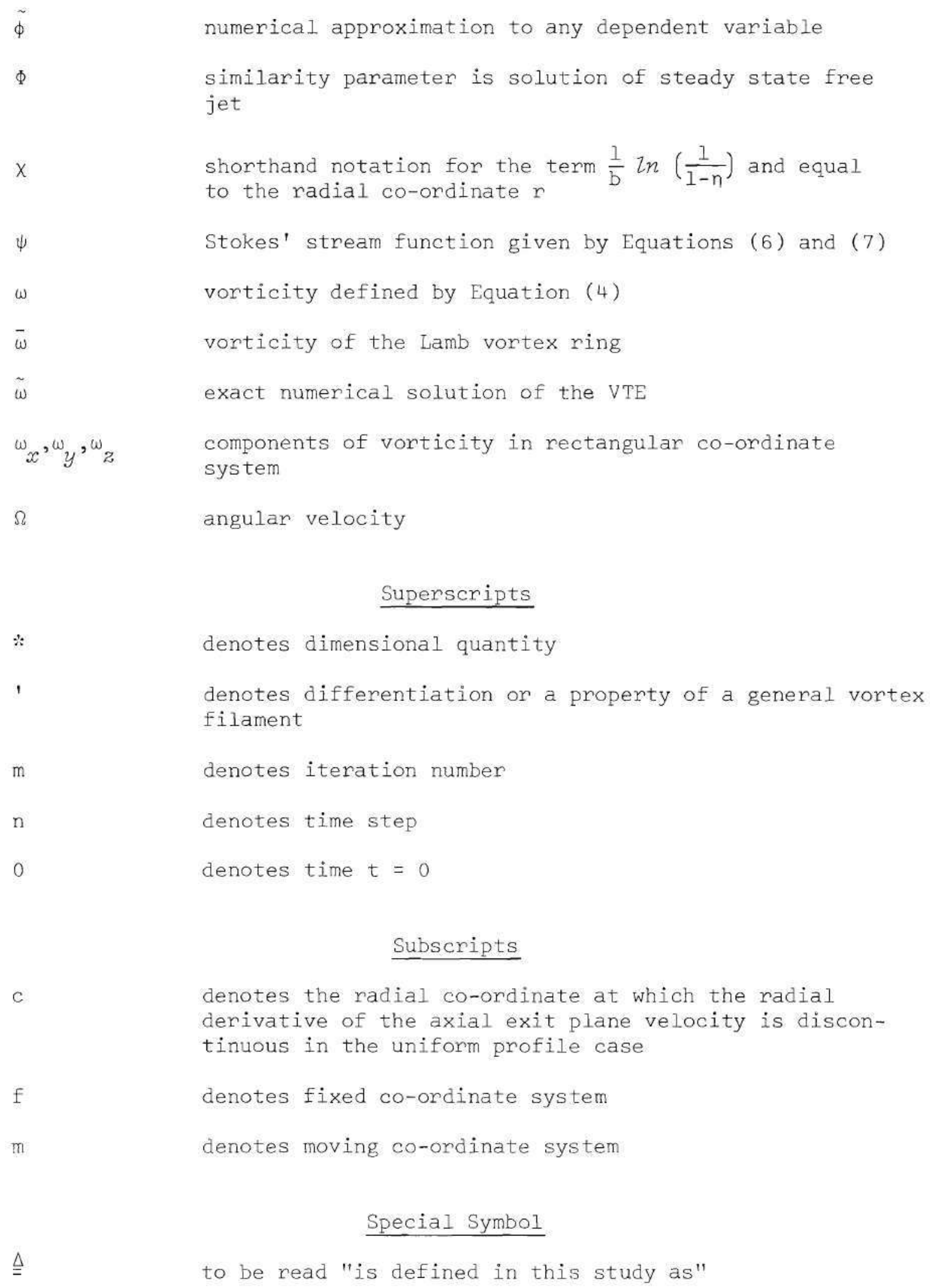

numerical approximation to any dependent variable similarity parameter is solution of steady state free jet shorthand notation for the term $\frac{1}{b} \ln \left(\frac{1}{1-n}\right)$ and equal
to the radial co-ordinate $r$

Stokes' stream function given by Equations (6) and (7)

vorticity defined by Equation (4)

vorticity of the Lamb vortex ring

exact numerical solution of the VTE

components of vorticity in rectangular co-ordinate system

angular velocity

Superscripts

denotes dimensional quantity

denotes differentiation or a property of a general vortex filament

denotes iteration number

denotes time step

denotes time $t=0$

\section{Subscripts}

denotes the radial co-ordinate at which the radial derivative of the axial exit plane velocity is discontinuous in the uniform profile case

denotes fixed co-ordinate system

denotes moving co-ordinate system

Special Symbol

to be read "is defined in this study as" 


\section{SUMMARY}

The generation and development of an axisymmetric, incompressible viscous vortex ring is investigated analytically. The unsteady flow field is generated from rest by an arbitrary impulse of air from an orifice in a wall of infinite extent.

The system of three governing equations (the Navier-Stokes equations and the continuity equation) is reduced to a system of two equations (the vorticity transport equation and the definition of vorticity equation) by introduction of the Stokes' stream function. In order to solve these coupled, partial differential equations numerically over the quarter-infinite region of interest, an exponential transformation is employed to reduce the quarter-infinite region to a finite one. The governing equations are approximated by implicit finite difference equations, and the resulting system is solved numerically. The numerical results are presented in terms of the vorticity and the stream function as functions of space and time. The velocity components may be calculated from the stream function distribution.

The numerical technique is investigated in detail for two cases. The two cases are the parabolic profile case and the uniform profile case which describe the specified initial impulse velocity in the orifice. First, a convergence study for each case is conducted using two different mesh sizes, and the comparison of results obtained with the two meshes is good. The numerical model is verified further by 
demonstrating that it accurately preserves an exact steady state solution to the boundary layer equations for axisymmetric, incompressible flow from a small round hole.

The instantaneous streaklines, velocity vector fields, vorticity contours, and streamline patterns for both the uniform and parabolic profile cases show the development of a viscous vortex ring. The results offer a more detailed description of vortex ring generation than has been obtained by experimental flow visualization techniques, and they compare well with the limited experimental data available on the behavion of vortex rings. The streaklines of the initial stages of vortex development agree qualitatively with smoke pictures that have been obtained experimentally. The results of the present investigation indicate that the region of high vorticity near the vortex center extends over a large region of the flow.

The numerical results for the parabolic profile case are analyzed to study the characteristics of the developed vortex ring. The radius, trajectory, and velocity of the ring are calculated, and the circulation along streamlines about the vortex is computed in a coordinate system fixed in space and in a co-ordinate system moving with the speed of the vortex. In turn, these parameters are employed to predict the propagation velocity of the vortex ring using the vortex models of Lamb and Hill. The vortex ring proposed by Lamb which consists of a small, circular rotational core embedded in an irrotational field is not an accurate model for the viscous vortex ring of this study. On the other hand, the comparison of the propagation velocities with Hill's spherical vortex is good, and at any given time, the ring 
velocity compares within about 15 per cent with that predicted by the steady state spherical vortex. 
CHAPTER I

\section{INTRODUCTION}

An interest in the phenomena of vortices has existed for well over a century. Although some of the initial studies were rigorous but academic, modern technology has recognized the practical need for the solution of a number of important problems of aerodynamics and the need for a fundamental understanding of the vortices which comprise these flow fields.

The earliest discussions appeared over a century ago in the works of Helmholtz [1], Stokes [2,3], Reynolds [4], J. J. Thompson [5], and Sir W. Thompson [6]. Lamb [7] and Basset [8] present a detailed review of these classical theories of inviscid, incompressible flow. A more recent but less exhaustive treatment is given by Ringleb [9]. The primary motivation of many of these initial studies was an academic interest in the physics and mathematics of the vortex. It is from this viewpoint that Lamb presents the details of a discussion given in an earlier paper of one of the few analyses of a vortex ring.

One of the characteristics of the classical vortex is the infinite velocity at its center. To avoid this difficulty and to help explain the action of viscosity at the vortex center, Lamb adopts a model the center of which is a small but finite core which rotates as a solid body. By assuming that the radius of the core is much smaller than the radius of the ring, he predicts the velocity, impulse, and energy of the ring in terms of a number of parameters. One of these is 
the unknown core radius. Unfortunately, Lamb gives no indication of the size of the core, nor does he venture an opinion concerning the validity of his model as an approximation to a physically real phenomenon.

Helmholtz introduced his vortex theorems in 1858, and the parallel development of electrodynamic and hydrodynamic theory of that period produced the Biot-Savart Law which remains to this day as an accurate and versatile method by which flow fields may be mathematically represented. However, other interests in vortices have grown and diminished during the period extending from the mid-nineteenth century to today. Kelvin [10] postulated the structure of the atom to be that of a vortex ring, and no doubt the failure of this idea diminished confidence in the utility of vortex theory. Nevertheless, Kutta [11] and Joukowski [12] firmly demonstrated its worth when they were able to predict the lift on an airfoil.

Phenomena associated with viscous vortex flows are currently receiving attention. Hall [13] has investigated the leading edge vortex of a wing by employing a method familiar to boundary layer analysts. He divided the flow field into two regions of interest, an inner region in which viscous effects are relatively important and an outer region where they may be neglected. In connection with application to the problems of nuclear propulsive devices, Donaldson and Sullivan [14] have found solutions to the Navier-Stokes equations for a complete class of threedimensional vortices, although, as pointed out by Rosenzweig, Lewellen, and Ross [15] in the study of a similar problem, these solutions are not readily applicable to flow in an actual vortex tube because of the dif- 
ficulty encountered in applying the boundary conditions. In search of further applications to nuclear propulsive devices, Lewellen [16] has found solutions to a three-dimensional confined vortex by linearizing the Navier-Stokes equations and by considering perturbations about both simple non-rotating flows and strongly rotating flows.

A number of solutions to various vortex flows have been obtained by applying numerical methods to the Navier-Stokes equations. Among the earliest of these was the work of Payne [17] who investigated the starting and perturbation of a two-dimensional, incompressible jet at low Reynolds numbers. Recently, at the Los Alamos Scientific Laboratory, Fromm [18] has studied the time dependent vortex formation behind a blunt body for incompressible two-dimensional flow, and Harlow, Shannon, and Welch [19] have been analyzing a number of problems concerning the unsteady flow of a fluid having a free surface. Greenspan [20] and his co-workers at the University of Wisconsin have been studying general numerical methods for solving both steady and unsteady incompressible vortex flows as well as comparing their solutions with other published results. Both Pearson [21] and Esch [22] have also been studying numerical solutions to a particular confined vortex of unsteady, incompressible flow. The experience of all these investigators shows numerical analysis to be a feasible and desirable method of solution for the Navier-Stokes equations.

Also of current interest are many of the problems of aerodynamics whose analyses are based on the assumption of an inviscid, incompressible medium. In formulating these types of flows, a classical approach is very often used in which the flow field is built up by the super- 
position of both free and bound vortices and vortex sheets. In theory no problem arises in calculating the effect of one vortex filament on another, but if a finite self-induced velocity is desired, then the geometry of the cross-section of the rotational core must be known. In other words, in order to avoid the infinite velocity associated with a line vortex, the mathematical model of the vortex must include a rotational core. This is essential to the low-speed flows of hovering helicopter rotors, propellers in static thrust, and take-off of V/STOL aircraft for here the self-induced velocity will largely determine the geometry of the vortex model of the system.

Using an inviscid, incompressible fluid, a number of investigators have studied vortex flows in order to analyze blade loading and the geometry of helicopter wakes. In the work of Brady and Crimi [23], Crimi [24], and DuWaldt [25], the helicopter flow field was simulated either by a system of discrete vortex rings or by a helical vortex shed from the blade tip. To avoid the difficulties associated with the infinite velocity of the vortex center which is encountered in the computation of the self-induced velocity of the vortex, they employed one of the few analytical tools available, the Lamb vortex ring. Brady and Crimi [23] concluded that the initial core size took a representative value of 5 per cent of the rotor radius and that their results did not change appreciably when the value of the core size varied over a range of from 0.265 to 14 per cent of the rotor radius. DuWaldt [25] was able to estimate the same representative value of the core size from energy considerations. Another study by Adams [26] established a core size of about 10 per cent of the rotor blade chord. Although this is a substan- 
tial difference from the results of Brady and Crimi [23] and DuWaldt [25], it may be relatively unimportant in that portion of the wake which is far removed from the rotor plane. However, both Adams [26] and Gray [27] have shown that the cores of the rotor tip vortices pass above and then down through the rotor plane for the case of a helicopter in forward flight. This action can have great effect both on the wake distribution near the rotor and on blade loading. Although the core may not pass through the rotor plane of a hovering helicopter, the proximity of the vortex to the rotor plane immediately after it is shed can have great influence on the loading of succeeding blades. Simons [28] has shown analytically that even the existence of a finite core affects blade loading. Apparently then, there exists some doubt about the size of the vortex core, and it appears that near the rotor plane this is a very important parameter.

In spite of the proven success of using vortices to determine aerodynamic loadings, very little is known concerning the structure of the vortex. It has already been reported in the previous paragraph that in a number of cases, a precise knowledge of this structure is relatively unimportant. This fact has probably obscured the need for research into the fundamental behavior of the vortex. However, some experimental work has been done in the investigation of a vortex ring. Unfortunately, much of this has been directed toward overall flow characteristics using flow visualization techniques; hence, only qualitative findings can be discussed. Banerji and Baravi [29] in 1931 and Okabe and Inoue [30] in 1960 
investigated the vortex phenomenon which is effected by forcing a small amount of fluid from a generator tube into a very large volume of the same medium. In both these studies colored water was ejected into a large tank of water. The qualitative results are illustrated in Figure 1.

Initially, the interface between the ejected fluid and the quiescent surroundings tends to flatten out, much like a rivet head, and then to curl axisymmetrically outward drawing ambient fluid into the center of the swirl. This is shown in Figure 1-a and Figure 1-b. During the rolling up process, the swirl continues to grow tighter drawing more fluid to its center, and the free end of the fluid layer near the center of the swirl appears to move through the ambient surroundings with considerable velocity. Figure $1-c$ shows the column which extends from the tube to the vortex breaking and being pulled into the rotational motion of the vortex. Finally, in Figure l-d, the vortex closes upon itself. According to Okabe and Inoue [30] the vortex ring is completed when a hole appears at the center of the ring cross-section, Figure 1-e.

Margavey and MacLatchy [31] in 1964 conducted similar experiments in air. Although they concur with most of the findings illustrated in Figure 1, they feel that the dynamic mass of the fluid lacks the geometrical characteristics of a true ring, and their photographic evidence does not support the theory of the existence of the closed torroidal surface of the true ring. What they saw is illustrated in Figure 1-d. In further investigations they concluded:

The vortex was formed by ejecting a puff of colorless air from the tip of a vertical tube close to which was rising a fine thread of smoke. Many photographs of this type have been 


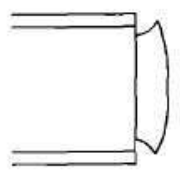

a

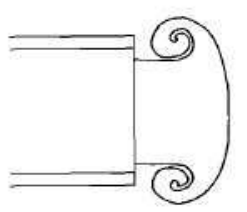

b

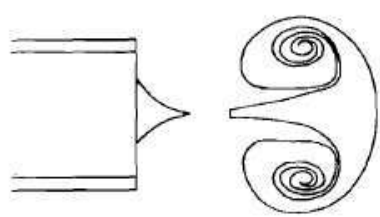

c
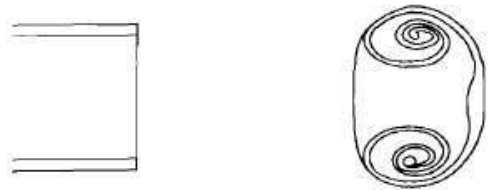

d
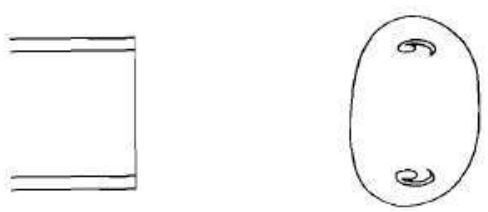

e

Figure 1. Formation of a Viscous Vortex Ring 
examined in detail and there appears to be no evidence of a closed stream surface defining a ring and no indication of coaxial stream surfaces in accordance with the concept of the classical model.

However, it is important to note here that only instantaneous streaklines can be observed by flow visualization techniques, and little can be said about streamlines or stream surfaces. In spite of this discrepancy though, there appears to be some disagreement between the findings of Margavey and MacLatchy [31] and those of other investigators [29,30] especially concerning the latter stages of vortex ring formation. It is the purpose of this study to investigate analytically a vortex ring which is generated in the same manner as those produced by Banerji and Baravi [29], Okabe and Inoue [30], and Margavey and MacLatchy [31]. This research is conducted in a number of steps. First, a mathematical model is derived for a physical system which is very similar to those of the previously cited experimental work, and a method is developed for the numerical solution of the incompressible Navier-Stokes equations. Next, the generation of a viscous vortex ring is simulated by the numerical model, and the resulting behavior of the ring is discussed. Finally, the numerical results are compared with those of existing, applicable, simplified vortex flows. 
CHAPTER II

THEORETICAL FORMULATION

In this chapter, a simplified physical model is adopted for the analytical investigation of the generation and development of an incompressible, axisymmetric viscous vortex ring. Next, the governing equations are introduced, and a mathematical model is derived which is amenable to solution by numerical methods.

The Physical Model

To facilitate the present analytical work, two simplifying assumptions are made. First, the phenomenon of vortex ring generation is assumed to be axisymmetric about the extended centerline of the generator tube. The simpler analyses of Lamb and others to which the results of this investigation will be compared are also axisymmetric, and experimental results indicate that a carefully produced vortex ring is axisymmetric. Second, it is assumed that the generator tube is one with walls of infinite thickness. This physical model is shown in Figure 2. The generator is now simply a hole in a plane of infinite extent. This assumption serves to limit the control volume to a quarterinfinite region extending from the exit plane and the solid wall to infinity in the axial direction and from the center line to infinity in the radial direction. 


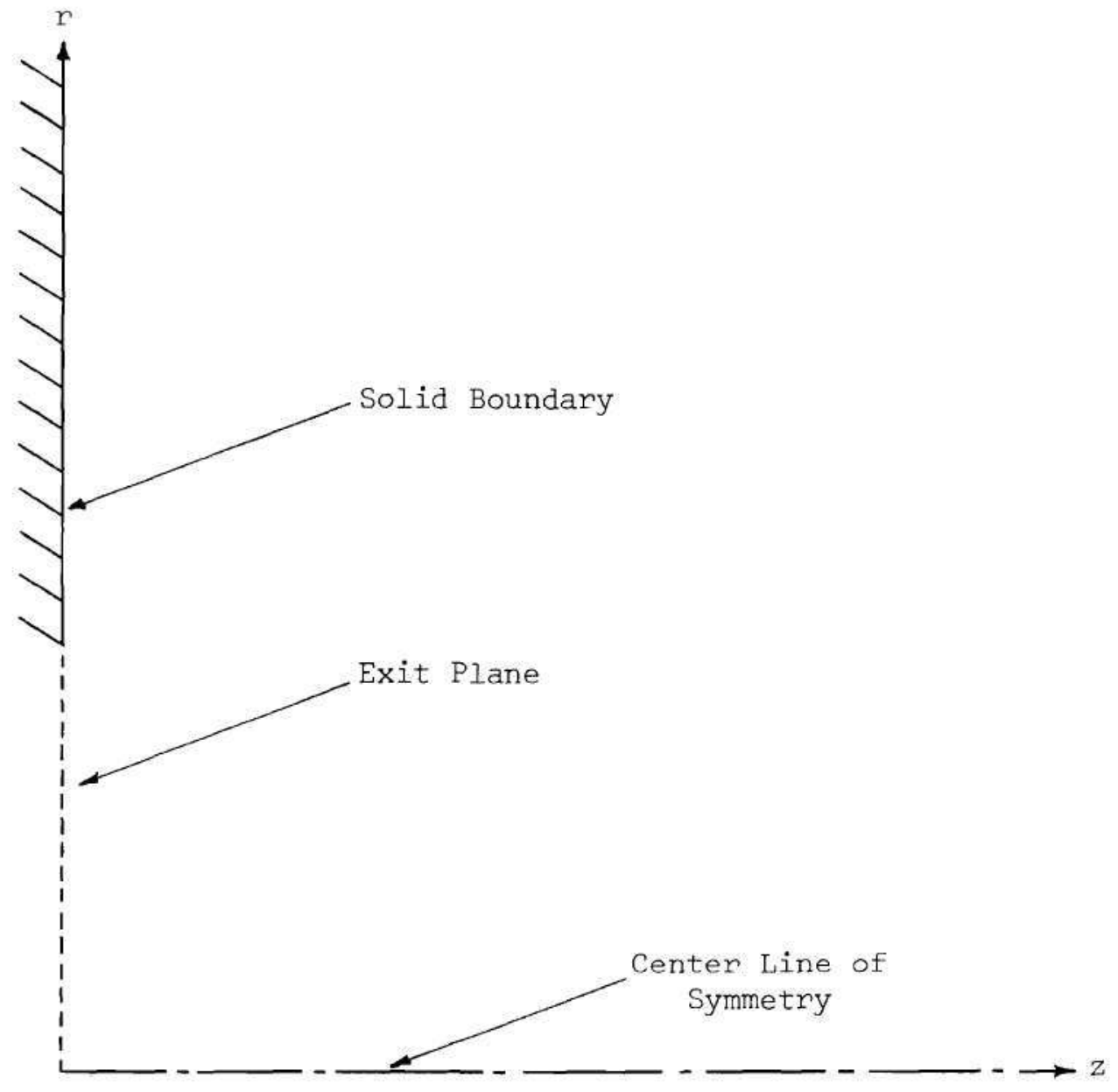

Figure 2. Geometry of the Physical Model 
The Mathematical Model

The equations governing the time dependent generation of a three-dimensional, axisymmetric vortex ring in an incompressible, viscous fluid are the Navier-Stokes equations and the continuity equation which may be written as

$$
\begin{gathered}
\frac{\partial v_{z}^{*}}{\partial t^{*}}+v_{z}^{*} \frac{\partial v_{z}^{*}}{\partial z^{*}}+v_{r}^{*} \frac{\partial v_{z}^{*}}{\partial r^{*}}=-\frac{1}{\rho^{*}} \frac{\partial p^{*}}{\partial z^{*}}+v^{*}\left\{\frac{\partial^{2} v_{z}^{*}}{\partial z^{* 2}}+\frac{1}{r^{*}} \frac{\partial v_{z}^{*}}{\partial r^{*}}+\frac{\partial^{2} v_{z}^{*}}{\partial r^{* 2}}\right\} \\
\frac{\partial v_{r}^{*}}{\partial t^{*}}+v_{z}^{*} \frac{\partial v_{r}^{*}}{\partial z^{*}}+v_{r}^{*} \frac{\partial v_{r}^{*}}{\partial r^{*}}=-\frac{1}{\rho^{*}} \frac{\partial p^{*}}{\partial r^{*}}+v^{*}\left\{\frac{\partial^{2} v_{r}^{*}}{\partial r^{* 2}}+\frac{\partial}{\partial r^{*}}\left(\frac{v_{r}^{*}}{r^{*}}\right)+\frac{\partial^{2} v_{r}^{*}}{\partial r^{* 2}}\right\} \\
\frac{\partial v_{z}^{*}}{\partial z^{*}}+\frac{v_{r}^{*}}{r^{*}}+\frac{\partial v_{r}^{*}}{\partial r^{*}}=0
\end{gathered}
$$

This dimensional system describes the three dependent variables $\left(v_{z}^{*}, v_{r}^{*}, p^{*}\right)$ in terms of the three independent variables $\left(t^{*}, z^{*}, r^{*}\right)$ and two properties of the fluid $\left(\nu^{*}, \rho^{*}\right)$. The solution of the equivalent dimensionless equations is an initial value problem requiring a known distribution of $v_{z}(t, z, r), v_{r}(t, z, r)$, and $p(t, z, r)$ at some initial time as well as boundary conditions for the unknowns at all subsequent times. A particular boundary condition may be expressed as either (I) the value of the unknown at the boundary (the Dirichlet condition), (2) the value of the normal derivative of the unknown at the boundary (the Neumann condition), or (3) some algebraic combination of the two (the Newton condition). The velocity boundary conditions may be stated 
quite simply on all but one of the boundaries. Both velocity components may be specified to vanish at large distances from the origin for physical reasons. If these velocities are not zero, the physical system possesses infinite energy. On the solid boundary the velocities vanish due to the no-slip condition. In the exit plane the radial velocity component is taken as zero, and the axial component is to be specified. At the center line, the radial velocity is zero due to symmetry, but nothing can be said concerning the value of the axial velocity component, so the Dirichlet condition cannot be applied. The radial derivative of the axial velocity is zero at the center line due to symmetry, but application of this Neumann condition presents difficulties when used with the finite difference method. Although various attempts were made in this research to solve a system of equations derived from the Navier-Stokes equations with boundary conditions expressed in terms of velocities, none proved fruitful. A discussion of these attempts may be found in Appendix A.

The governing equations are now manipulated to a form for which the boundary conditions can be more clearly stated and applied. In so doing, an additional advantage occurs. The system may be reduced to two equations in two unknowns by introducing the stream function, $\psi *$ Differentiating Equation (1) with respect to $r^{*}$ and Equation (2) with respect to $z^{*}$, taking the difference of the two resulting equations, and employing the definition of vorticity equation, hereafter referred to as the DVE,

$$
\omega^{*} \triangleq \frac{\partial v_{r}^{*}}{\partial z^{*}}-\frac{\partial v^{*}}{\partial r^{*}}
$$


allows the elimination of the pressure $p^{*}$ and the derivation of the vorticity transport equation, hereafter referred to as the VTE,

$$
\frac{\partial \omega^{*}}{\partial t^{*}}+\frac{\partial\left(v_{z}^{* *}\right)}{\partial z^{*}}+\frac{\partial\left(v_{p}^{*} \omega^{*}\right)}{\partial r^{*}}=\nu^{*}\left\{\frac{\partial^{2} \omega^{*}}{\partial z^{* 2}}+\frac{1}{r^{*}} \frac{\partial \omega^{*}}{\partial r^{*}}-\frac{\omega^{*}}{r^{* 2}}+\frac{\partial^{2} \omega^{*}}{\partial r^{* 2}}\right\}
$$

Introducing the Stokes' stream function, given by

$$
v_{z}^{*}=\frac{1}{r^{*}} \frac{\partial \psi^{*}}{\partial r^{*}}
$$

and

$$
v_{r}^{*}=-\frac{1}{r^{*}} \frac{\partial \psi^{*}}{\partial z^{*}}
$$

into Equation (5) and Equation (4) yields two simultaneous, non-linear, partial differential equations. The VTE now takes the form

$$
\begin{aligned}
& \frac{\partial \omega^{*}}{\partial t^{*}}+\frac{\partial}{\partial z^{*}}\left\{\frac{1}{r *} \frac{\partial \psi^{*}}{\partial r^{*}} \omega^{*}\right\}+\frac{\partial}{\partial r^{*}}\left\{-\frac{1}{r^{*}} \frac{\partial \psi^{*}}{\partial z^{*}} \omega^{*}\right\}=\nu^{*}\left\{\frac{\partial^{2} \omega^{*}}{\partial z^{* 2}}\right. \\
& \left.+\frac{1}{r *} \frac{\partial \omega^{*}}{\partial r^{*}}-\frac{\omega^{*}}{r^{* 2}}+\frac{\partial^{2} \omega^{*}}{\partial r^{* 2}}\right\}
\end{aligned}
$$

and the DVE now takes the form

$$
\frac{1}{r^{*}} \frac{\partial^{2} \psi^{*}}{\partial z^{* 2}}-\frac{1}{r^{* 2}} \frac{\partial \psi^{*}}{\partial r^{*}}+\frac{1}{r^{*}} \frac{\partial \psi^{*}}{\partial r^{* 2}}=-\omega^{*}
$$


in the unknowns $\omega^{*}$ and $\psi^{*}$.

It may be interesting to note at this time that Equation (9) may be substituted into Equation ( 8 ) to produce a complicated fourth order, non-linear, partial differential equation in the unknown $\psi^{*}$. However, the experience of Pearson [32] indicates that a numerical solution of this fourth order equation is less rewarding than that of the coupled Equations (8) and (9).

The governing equations may be written in dimensionless form by employing various reference parameters. In this research, use will be made of the dimensionless variables,

$$
\begin{aligned}
& t=\frac{t^{*} U}{R} \quad r=\frac{r^{*}}{R} \quad z=\frac{z^{*}}{R} \\
& \omega=\frac{\omega^{*} \mathrm{R}}{\mathrm{U}} \quad \mathrm{v}_{\mathrm{r}}=\frac{\mathrm{v}_{\mathrm{r}}^{*}}{\mathrm{U}} \quad \mathrm{v}_{\mathrm{z}}=\frac{\mathrm{v}_{\mathrm{z}}^{*}}{\mathrm{U}} \quad \psi=\frac{\psi^{*}}{\mathrm{R}^{2} \mathrm{U}}
\end{aligned}
$$

where $R$ and $U$ are a convenient reference length and velocity. Employing these reference parameters yields the governing dimensionless equations, the VTE

$$
\frac{\partial \omega}{\partial t}+\frac{\partial\left(v_{z} \omega\right)}{\partial z}+\frac{\partial\left(v_{r} \omega\right)}{\partial r}=\frac{1}{R e y}\left\{\frac{\partial^{2} \omega}{\partial z^{2}}+\frac{1}{r} \frac{\partial \omega}{\partial r}-\frac{\omega}{r^{2}}+\frac{\partial^{2} \omega}{\partial r^{2}}\right\}
$$

the DVE,

$$
\frac{1}{r} \frac{\partial^{2} \psi}{\partial z^{2}}-\frac{1}{r^{2}} \frac{\partial \psi}{\partial r}+\frac{1}{r} \frac{\partial^{2} \psi}{\partial r^{2}}=-w
$$


and the definition of the stream function,

$$
\begin{aligned}
& v_{z}=\frac{1}{r} \frac{\partial \psi}{\partial r} \\
& v_{r}=-\frac{1}{r} \frac{\partial \psi}{\partial z}
\end{aligned}
$$

where

$$
\text { Rey }=\frac{\mathrm{UR}}{v^{*}}
$$

is the Reynolds number. Esch [22] has formulated a similar problem using different dimensionless variables to obtain a form of the VTE which is independent of the Reynolds number. Instead, the Reynolds number appears in the boundary conditions so that no apparent advantage results, at least for this particular problem.

All the initial and boundary conditions needed for the solution of the system composed of Equations (11) and (12) can be deduced from the physical consideration of the components of velocity. At great distances from the origin of the co-ordinate system, the velocities and their derivatives must vanish. Unless this is the case, the energy of the physical system is infinite. On the solid wall the velocities vanish because of the no-slip condition, and in the exit plane the velocities will be specified. On the center line, conditions of symmetry exist which allow evaluation of certain velocity characteristics. Since the VTE, Equation (11), is parabolic in time its solution 
requires an initial condition on $\psi$ and $\omega$ at some convenient time $t$ and boundary conditions on $w$ at all succeeding times. At the initial time the velocities vanish, so the initial condition is that $\psi$ and $\omega$ are zero. Thus,

for $t=0$

$$
\omega=\psi=0
$$

Consider now the boundary conditions for the VTE. At the center line, a line of symmetry, observation of Equation (4) indicates that the vorticity vanishes since both the derivatives comprising the vorticity vanish, the first because the radial velocity is zero all along the center line due to symmetry, and the second because the radial derivative of the axial velocity is zero again due to symmetry. Hence,

for $r=0$

$$
\omega=0
$$

As the spatial co-ordinates become very large, the velocity derivatives vanish. Thus,

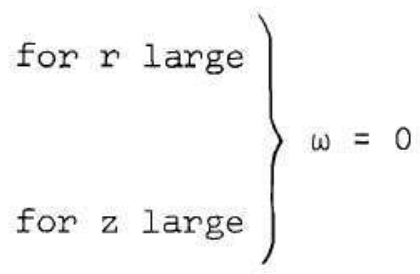

In the exit plane, the axial velocity component will be given a specified distribution, and consequently its radial derivative will be known. On the solid boundary, the no-slip condition requires that the axial and 
radial velocity components be zero, and the radial derivative of the axial velocity must vanish. In contrast, the axial derivative of the radial velocity component is not known, nor can it be easily specified. Therefore, in order to compute this derivative, some knowledge of its behavior must be ascertained. If the reference length is now chosen as the radius of the exit plane, the boundary conditions in the exit plane and on the solid boundary become

$$
\omega=\frac{\partial v_{r}}{\partial z}-\frac{\partial v_{z}}{\partial r}
$$

where for

$$
\left.\begin{array}{l}
\text { (1) } r \leq 1, \frac{\partial v}{\partial r} \text { is specified } \\
\text { (2) } r>1, \frac{\partial v}{\partial r}=0
\end{array}\right\}
$$

(3) $0 \leq r \leq \infty, \frac{\partial v_{r}}{\partial z}$ is yet to be determined.

Equations (15), (16), (17), (18), (19), and (20) completely specify the initial condition and the boundary conditions needed for the solution of the VTE.

The DVE, Equation (12), is elliptic and must be solved at each time step after the corresponding vorticity distribution has been computed from the VTE. Thus, boundary conditions on the stream function must be specified for all time. The center line, a line of symmetry, 
is a streamline, and since the stream function may be specified within an arbitrary constant, the stream function at the center line is chosen as some convenient reference quantity. Thus,

for $n=0$

$$
\psi=0
$$

In the exit plane the axial velocity component is specified. This enables evaluation of the stream function both in the exit plane and on the solid boundary by integration of Equation (13). Thus, at any time

for $\mathrm{z}=0$

$$
\psi(0, r)=\int_{0}^{r} v_{z}(0, r) r d r
$$

where $v_{z}(0, r)$ either has a known distribution in the exit plane $(r<1)$ or vanishes on the solid boundary $(r \geq 1)$. It can be seen from Equation (22) that since $v_{z}$ vanishes on the solid wall, the stream function is constant over this region. On the remaining boundaries where either the axial or radial spatial co-ordinate becomes very large, the boundary condition on the stream function may be specified as a Neumann condition. The radial velocity is given by

$$
v_{r}=-\frac{1}{r} \frac{\partial \psi}{\partial z}
$$

and for large values of $\mathrm{z}$, a vanishing radial velocity requires that

for z large

$$
\frac{\partial \psi}{\partial z}=0
$$


The axial velocity is given by

$$
\mathrm{v}_{\mathrm{z}}=\frac{1}{r} \frac{\partial \psi}{\partial r}
$$

and for large values of $r$, a vanishing axial velocity requires that

for $r$ large

$$
\frac{\partial \psi}{\partial r}=0
$$

Equations (21), (22), (23), and (24) completely specify the boundary conditions needed for the solution of the DVE.

The governing equations which describe the behavior of the fluid in an infinite flow field must now be simulated by a mesh composed of a finite number of grid points. This necessitates the transformation of the infinite region of interest to a finite one. An exponential transformation is proposed of the form *

$$
\begin{aligned}
& \xi=1-e^{-a z} \\
& n=1-e^{-b r}
\end{aligned}
$$

where $\mathrm{a}$ and $\mathrm{b}$ are convenient constants or "stretching factors." That the transformation does indeed map an infinite region into a finite

*The author gratefully acknowledges the suggestion of this type of transformation by his colleague, Dr. Jerry A. Sills. 
one can be seen by investigation of the limiting values of the spatial variables in the original and in the transformed plane. Thus

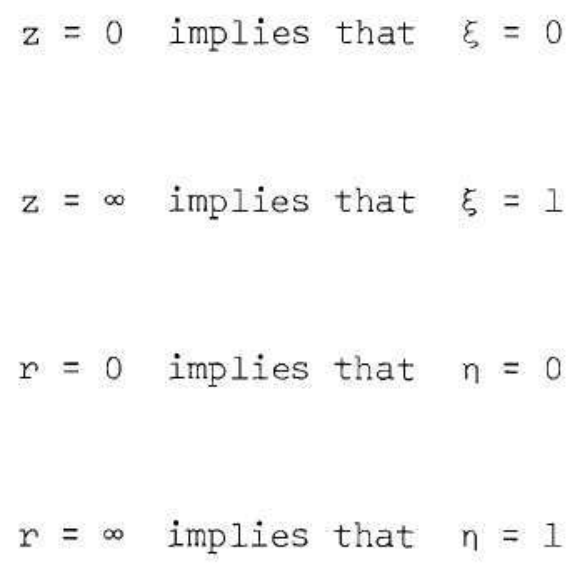

Fortunately, an additional advantage is realized. An evenly spaced grid in the $\xi, n$ plane transforms to an unevenly spaced grid in the $z, r$ plane, and the grid spacing in the $\mathrm{z}, \mathrm{r}$ plane is smallest in the region in which the flow field is changing fastest, i.e., where $z$ and $r$ are small. Differentiation and rearrangement of Equations (25) and (26) permit computation of the various differential operators needed. These become

$$
\begin{aligned}
& \frac{\partial}{\partial z}=a(1-\xi) \frac{\partial}{\partial \xi} \\
& \frac{\partial^{2}}{\partial z^{2}}=a^{2}(1-\xi)^{2} \frac{\partial^{2}}{\partial \xi^{2}}-a^{2}(1-\xi) \frac{\partial}{\partial \xi} \\
& \frac{\partial}{\partial r}=b(1-\eta) \frac{\partial}{\partial \eta} \\
& \frac{\partial^{2}}{\partial r^{2}}=b^{2}(1-\eta)^{2} \frac{\partial^{2}}{\partial \eta^{2}}-b^{2}(1-\eta) \frac{\partial}{\partial \eta}
\end{aligned}
$$


Employing these operators on the Equations (11), (12), (13), and (14) yields the governing equations in the transformed plane which are the VTE,

$$
\begin{aligned}
& \frac{\partial \omega}{\partial t}+a(1-\xi) \frac{\partial\left(v_{z} \omega\right)}{\partial \xi}+b(1-n) \frac{\partial\left(v_{r} \omega\right)}{\partial \eta}= \\
& \frac{1}{R e y}\left\{a^{2}(1-\xi)^{2} \frac{\partial^{2} \omega}{\partial \xi^{2}}-a^{2}(1-\xi) \frac{\partial \omega}{\partial \xi}+\frac{b(1-\eta)}{x} \frac{\partial \omega}{\partial \eta}-\frac{\omega}{x^{2}}\right. \\
& \left.+b^{2}(1-n)^{2} \frac{\partial^{2} \omega}{\partial \eta^{2}}-b^{2}(1-n) \frac{\partial \omega}{\partial \eta}\right\}
\end{aligned}
$$

the DVE,

$$
\begin{aligned}
& \frac{1}{x}\left\{a^{2}(1-\xi)^{2} \frac{\partial^{2} \psi}{\partial \xi^{2}}-a^{2}(1-\xi) \frac{\partial \psi}{\partial \xi}\right\}-\frac{1}{x^{2}}\left\{b(1-n) \frac{\partial \psi}{\partial \eta}\right\} \\
& +\frac{1}{x}\left\{b^{2}(1-n)^{2} \frac{\partial^{2} \psi}{\partial \eta^{2}}-b^{2}(1-n) \frac{\partial \psi}{\partial \eta}\right\}=-\omega
\end{aligned}
$$

and the definition of the stream function,

$$
\begin{aligned}
& v_{z}=\frac{1}{x} b(1-n) \frac{\partial \psi}{\partial n} \\
& v_{r}=-\frac{1}{x} a(1-\xi) \frac{\partial \psi}{\partial \xi}
\end{aligned}
$$

where 


$$
r=x \triangleq \frac{1}{b} \ln \left(\frac{1}{1-n}\right)
$$

has been adopted as a short hand notation. Recall that in the computational procedure Equations (33) and (34) will be substituted into Equation (31) to yield again two equations, the VTE and the DVE, in the two unknowns, $\omega$ and $\psi$. In the transformed plane the VTE remains parabolic in time, and the DVE is elliptic. The initial condition remains the same in the transformed plane; the fluid is at rest. Thus,

$$
\omega(0, \xi, n)=\psi(0, \xi, n)=0
$$

The boundary conditions on the VTE become

$$
\begin{aligned}
& \omega(t, \xi, 0)=0 \\
& \omega(t, \xi, 1)=0 \\
& \omega(t, I, \eta)=0
\end{aligned}
$$

and

$$
\omega(t, 0, n)=a \frac{\partial v_{r}}{\partial \xi}-b(1-n) \frac{\partial v_{z}}{\partial \eta}
$$

Adopting the notation that $n=n_{p}$ at $n=x=1$ shows that 
for $n \leq n_{P}, \quad b(1-n) \frac{\partial v_{z}}{\partial \eta}$ is specified.

$$
\text { for } n>n_{P}, \quad b(1-n) \frac{\partial v_{z}}{\partial n}=0
$$

and

$$
\text { for } 0 \leq n \leq 1, \quad \text { a } \frac{\partial v_{n}}{\partial \xi} \text { is yet to be determined. }
$$

Equations (35) through (42) specify in the transformed plane the initial condition and the boundary conditions needed for the solution of the VTE. Now consider the boundary conditions on the DVE, Equation (32). At the center line, a streamline, the stream function takes the same reference value,

$$
\psi(t, \xi, 0)=0
$$

In the exit plane and on the solid boundary, the stream function is determined to be

$$
\psi(t, 0, n)=\int_{0}^{n v_{z}(t, 0, n) x} \frac{z}{b(1-n)} d n
$$

The Neumann conditions on the normal derivatives of the stream function must also be prescribed in the transformed plane. Consider first the 
case for large $z$, or for $\xi$ approaching unity. The normal derivative of the stream function near this boundary may be written as

$$
\frac{\partial \psi}{\partial z}=a(1-\xi) \frac{\partial \psi}{\partial \xi}=0
$$

which implies the Neumann condition that

$$
\text { for } \xi \text { near } 1 \quad \frac{\partial \psi}{\partial \xi}=0
$$

Similarly, it may be specified that

$$
\text { for } n \text { near } 1 \quad \frac{\partial \psi}{\partial n}=0
$$

Equations (43), (44), (45), and (46) specify in the transformed plane the boundary conditions needed for the solution of the DVE.

At this point the governing equations as well as their initial and boundary conditions have been derived in a form convenient for solution by finite difference techniques. Once the VTE and DVE have been solved, the vorticity, the velocities, and the stream function are known functions of space and time. Although the pressure was eliminated as one of the dependent variables, a knowledge of its behavior may be desirable. For this reason, a method is presented in Appendix $B$ whereby the pressure may be computed at any time $t$ at which the other dependent variables are known. However, no pressure calculations were performed in this research. 
CHAPTER III

NUMERICAL FORMULATION AND VERIFICATION

At this point, the governing equations which describe the generation of an axisymmetric vortex ring in an incompressible, viscous fluid have been derived, and the initial and boundary conditions necessary for the solution of these equations have been developed. These equations and boundary conditions are expressed in a transformed plane of finite dimensions which is necessary for the implementation of a numerical solution.

This chapter presents discussions of the numerical method, some of the difficulties inherent in this method of solution, and techniques for surmounting these difficulties. Using these techniques, the governing equations and their accompanying initial and boundary conditions are expressed as finite difference equations. Finally, two particular problems are specified, the method of solution is indicated, and the numerical model is verified.

\section{The Method of Finite Differences}

Consider a partial differential equation that has been derived as the mathematical model which describes certain phenomena over some surface or volume of interest. A simple control surface is illustrated in Figure 3. When an exact method predicts a solution which is devoid of singular points, the solution applies continuously over the region of interest. That is, for any of the infinite number of points in the 


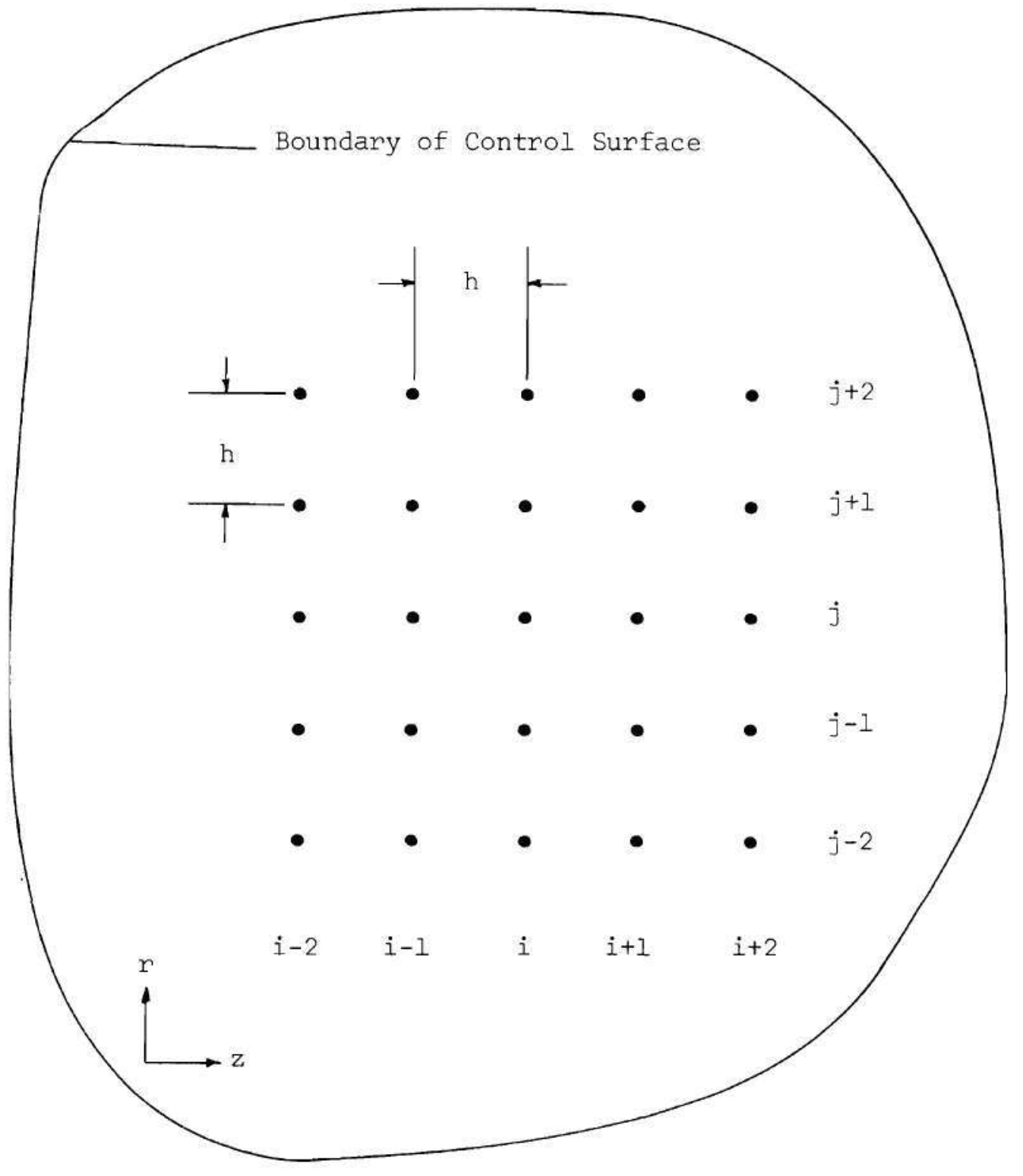

Figure 3. A Control Surface and a Portion of the Finite Difference Grid 
region, the solution is known for that particular point. However, if finding an exact solution is intractable, a number of other methods are available. Sometimes the equation may be transformed to an expression with one or two independent variables in which cases socalled similar or semi-similar solutions may be found. Alternatively, the solution may be assumed to be a series with undetermined coefficients in which case substitution of the series into the governing equation and simplification yields these coefficients and the series solution. The method of successive approximations allows the substitution of an initial guess at the solution into the governing equation after which some algorithm is applied to yield a better approximation. The integral method is another approximate procedure whereby the solution found satisfies some integral form of the original differential equation.

The approximate technique to be employed in this investigation is the finite difference method. Its popularity has grown considerably in recent years with the advent of electronic computers which are capable of handing the multitude of arithmetic operations and results. In addition, this method yields solutions to problems which often cannot be handled by any other technique. The rationale of the procedure is to approximate the exact solution at discrete points in the region of interest. Part of this system of points which is called alternately the mesh or the grid is also shown in Figure 3 . In order to approximate the solution at these discrete points, the governing equation must be approximated at these same points, and this in turn leads to simulating the particular terms of the differential equation on the grid. A number 
of finite difference approximations to various partial derivatives are derived in Appendix $\mathrm{C}$ along with the accompanying truncation errors. These errors in each derivative arise due to the truncation of Taylor series expansions which are employed in the derivations. When all of the derivatives of the partial differential equation have been replaced by finite difference approximations, there results an approximate difference equation at the general mesh point $(i, j)$. For the case of a second order partial differential equation, the finite difference approximation may be written in functional notation as

$$
G(i, j)=f\{G(i-1, j), G(i+1, j), G(i, j-1), G(i, j+1)\}
$$

where $G$ can be any dependent variable. When this difference equation is applied to all interior mesh points of the region of interest, a system of simultaneous algebraic equations results whose solution is taken to be an approximation to that of the differential equation. The finite difference method consists of three operations: (1) The derivation of the difference equation which approximates the original differential equation, (2) the solution of the resulting algebraic system, and (3) the verification of the solution. However, none of these operations can be divorced from the other two, and it will be demonstrated in the next section that evaluating the results is closely tied to deriving the model and solving it. 


\section{A Discussion of the Stability and Convergence of a Finite Difference Equation}

When a partial differential equation has been approximated by a well-posed finite difference equation, there results a system of simultaneous algebraic equations whose solution is assumed to be that of the original partial differential equation. How well the solution to the algebraic system approximates the solution to the differential equation is dependent upon a number of circumstances.

The existence of a unique solution is important from not only an academic but also a practical point of view; the solution which is found may not be the one which is sought. For the case of a non-linear partial differential equation in particular, little can be said concerning the uniqueness of the solution of either the algebraic system or the differential equation. This question is usually answered by the investigator who must apply his physical intuition to decide whether on not the function found by successive approximation is an acceptable solution. This is the procedure which will be followed in this research.

Two basic difficulties in finding a solution are liable to occur. To better understand these difficulties, a definition of terms is helpful. Let $D$ represent the exact solution to the partial differential equation, let $\Delta$ represent the exact solution to the finite difference equation, and let $\mathrm{N}$ represent the numerical solution to the difference equation. The difference $(\Delta-N)$ is called the numerical error, and it is the problem of stability to find a particular finite difference approximation to the differential equation such that the numerical error will remain small throughout the solution. The difference $(D-\Delta)$ is 
called the truncation error, and it is the problem of convergence to find the conditions under which $\Delta$ approaches D. It has already been mentioned and it can be seen in Appendix $C$ that the truncation error arises from the truncation of the Taylor series which is used in the approximation of each partial derivative.

The numerical instability of a finite difference equation is evidenced by the unbounded growth of numerical error, and the principal numerical error is computer round-off error. Instability is of prime concern when dealing with a parabolic equation which can be solved by a stepping procedure. It will be seen in the following pages that different but equal differential equations as well as a wide variety of finite difference analogs to the same differential equation all may give rise to unstable numerical models. The stability analysis of von Neumann as recorded by O'Brien, et al. [33] is used herein and is outlined briefly in the following discussion. Once a particular finite difference approximation has been selected, the equation must be linearized to conduct the stability analysis. It can be shown that for a linearized difference equation, the numerical error satisfies the same difference equation as does the particular dependent variable of interest. Next, the numerical error is assumed to have a typical Fourier component solution, and this solution is substituted into the difference equation whose stability is being analyzed. Analysis of the resulting equation then shows whether the error grows or diminishes for a general mesh point as the solution is advanced with respect to the stepping variable. Since a stability analysis can predict which finite difference representation offers promise, the development of the numerical analog to 
the VTE is based primarily upon stability. The simplest equation is investigated first, and the stability analysis dictates what changes must be made to obtain a satisfactory result. This procedure continues until an acceptable representation of the VTE has been derived and discussed.

In contrast to stability, convergence usually must be tested after a solution has been achieved. It can be seen from the finite difference approximations of Appendix $C$ that the truncation error of each difference approximation is a function of the mesh spacing. By decreasing the space between grid points, the truncation error for each partial derivative decreases, and in this manner the truncation error for the whole equation should decrease. This idea of shrinking the mesh spacing to decrease individual truncation errors provides the rationale for the following common method of demonstrating convergence. Assume that the relationship between mesh spacings has been retained but that the spacings between grid points have been steadily diminished in order to obtain a number of solutions to the same difference equation for finer and finer meshes. If this sequence of solutions tends toward a single solution, then the solution of the difference equation is said to have converged to the solution of the differential equation. This technique will be applied to the finite difference equations of this investigation.

Hildebrand [34] gives some additional encouragement for resolving this perplexing problem of convergence. He states:

As has already been pointed out, only scattered information is available as to general convergence of solutions of differenceequation problems to solutions of approximated differential- 
equation problems, with increasing net refinement. However, the existing evidence indicates that, if stability is attained at some stage of the refinement, then convergence generally follows when the prescribed functions involved in the end conditions and initial conditions are sufficiently well behaved. Whereas it has been shown that lack of stability does not inevitably imply lack of convergence, this result is of limited practical significance since instability generally renders a numerical procedure useless unless special methods of controlling propagated errors are employed.

Thus, existing evidence indicates that although only the investigation of a solution can indicate convergence, the presence of numerical stability increases the probability of its existence.

Since the stability and convergence of the governing equations are of prime concern in the numerical calculation of accurate results, the numerical analog of the VTE will be derived with the intention of obtaining a numerically stable equation. Stability is not of such great concern for the case of the DVE since this equation is linear. The numerical errors introduced into the DVE at each time step are random and additive, and the total numerical error always remains bounded for a finite number of time steps.

The Numerical Model

The purpose of the finite difference method is to compute the dependent variables, $\omega, \psi, v_{z}$, and $v_{r}$, at the grid points of a mesh in the trarsformed plane at the discrete time steps $n(\delta t)$ where $n$ is the time step index and $\delta t$ is the time step increment. The mesh spacing is that of Figure 4. For convenience the mesh is equally spaced in both the $\xi$ and $n$ directions with $M+1$ discrete points such that for $\xi=i / M$ and $n=j / M$, the mesh spacing is given by 


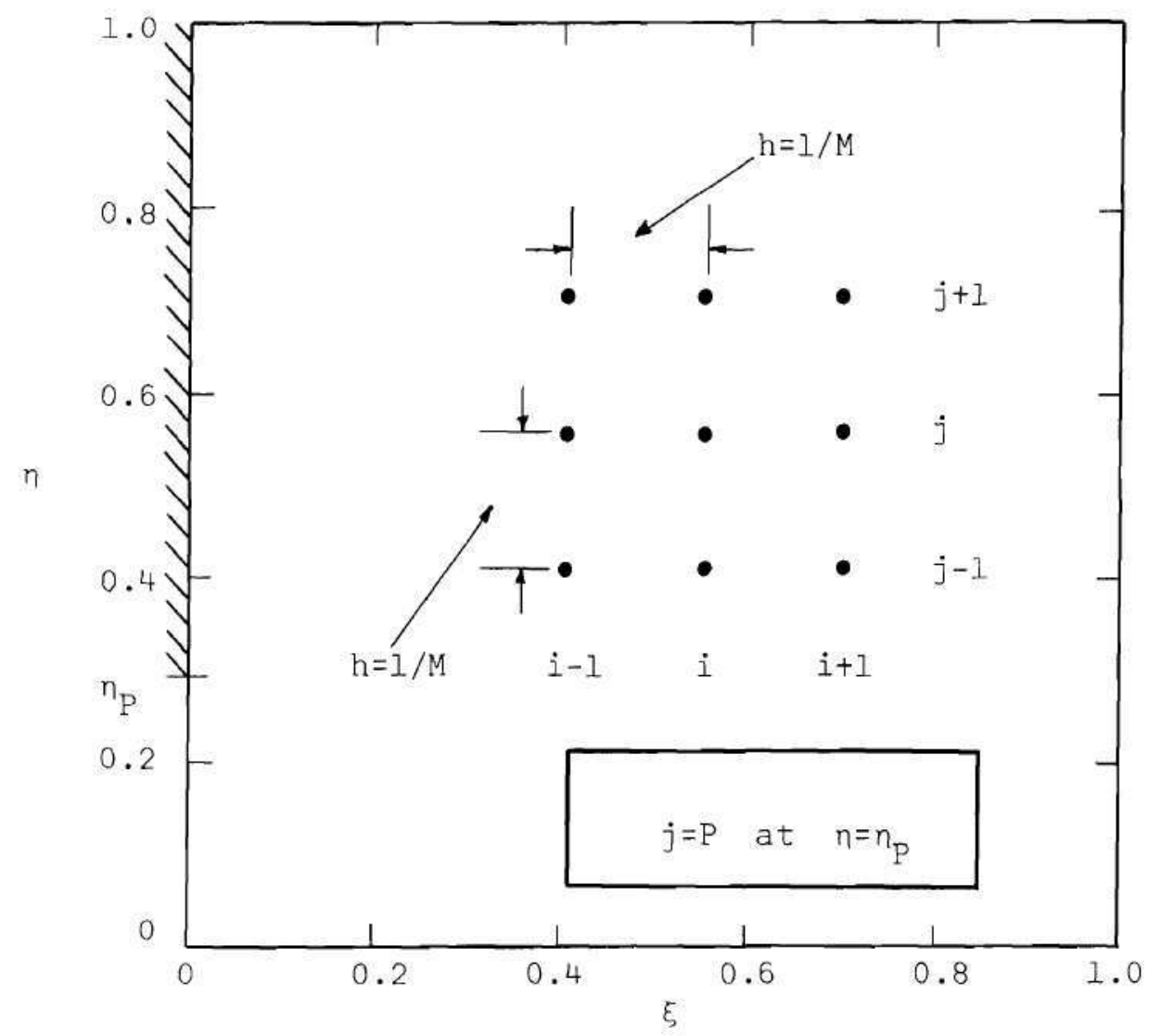

Figure 4. Mesh Spacing in the Transformed Plane 


$$
\Delta \xi=\Delta n=h=\frac{1}{M}
$$

The finite difference analog to the VTE, Equation (31), will then predict the vorticity at the new time at a given mesh point, $\omega^{n+l}(i, j)$, in terms of, among other things, the vorticity and stream function distributions, $\omega^{n}(i, j)$ and $\psi^{n}(i, j)$, at the previous time. With $\omega^{n+1}(i, j)$ now determined, the finite difference analog to the DVE, Equation (32), will predict the corresponding stream function distribution, $\psi^{n+1}(i, j)$, from which may be obtained the velocity distributions $v_{z}^{n+l}(i, j)$ and $v_{r}^{n+l}(i, j)$. At this point, it should be recalled that the boundary value of vorticity at $\xi=0$ at the new time must be determined from the velocity distribution through the use of Equation (39). The Finite Difference Analog to the VTE

It is sometimes important to write the finite difference expression for a partial differential equation in a conservative form. When this conservative form is used in the finite difference approximation and when the resulting equation is summed over the mesh, certain interior terms cancel in pairs. This cancellation may be seen for example in the approximation

$$
\frac{\partial\left(v_{z} \omega\right)}{\partial z}=\frac{v_{z}(i+1, j) \omega(i+1, j)-v_{z}(i-1, j) \omega(i-1, j)}{2 h}
$$

where $\mathrm{h}$ is the mesh spacing. When this partial derivative is summed over all $i$ for a particular $j$, the product $v_{z} \omega$ cancels at all mesh points $(i, j)$ except those on the boundaries $i=0$ and $i=M$ where they 
are specified. From [18] mentions that in the solution of a nonconservative equation, errors may occur which are associated with the residues remaining in the interior terms. However, Fromm's solution is explicit, and any error which occurs cannot be corrected. In an implicit technique, an iterative procedure is used, and the error associated with the interior terms can be corrected. In any case, the opportunity to state the VTE in a conservative form is now lost due to the transformation of the equation to the finite plane. Since the VTE cannot be stated conservatively, it may be desirable to employ the continuity equation to simplify the convective terms of the VTE. No additional difficulty arises from this non-conservative statement of the VTE since the transformation to the finite plane disallows any conservative statement of the VTE.

One may obtain a simplified form of the VTE, Equation (31), by expanding the convective terms, adding and subtracting the common term $\frac{v_{r}{ }^{\omega}}{x}$ to the convective terms, and employing continuity. The resulting equation may be written

$$
\begin{aligned}
& \frac{\partial \omega}{\partial t}+v_{z} a(1-\xi) \frac{\partial \omega}{\partial \xi}+v_{r}\left[b(1-n) \frac{\partial \omega}{\partial n}-\frac{\omega}{x}\right]= \\
& \frac{1}{R e y}\left\{a^{2}(1-\xi)^{2} \frac{\partial^{2} \omega}{\partial \xi^{2}}-a^{2}(1-\xi) \frac{\partial \omega}{\partial \xi}-\frac{\omega}{x^{2}}+\frac{b(1-n)}{x} \frac{\partial \omega}{\partial n}\right. \\
& \left.+b^{2}(1-n)^{2} \frac{\partial^{2} \omega}{\partial n^{2}}-b^{2}(1-n) \frac{\partial \omega}{\partial n}\right\}
\end{aligned}
$$


This expression is considered as an alternative to Equation (31) only because Equation (47) has a somewhat simpler form. A number of different finite difference analogs to Equations (31) and (47) may now be derived, thein chief difference being in the manner in which the time derivative is approximated. The stability of each analog is studied in Appendix D, and the difference equation itself is derived in Appendix E.

The first equation of interest is an explicit formulation of (47) using a forwand difference approximation to the time derivative. As shown in Appendix E, Case I, the finite difference equation simplifies to a finite difference expression in which the unknown parameter $\omega^{n+1}(i, j)$ is expressed in terms of the known vorticity and velocities at the time $n(\delta t)$. This is the simplest way to determine the new vorticity; the vorticity distribution at the time $(n+1) \delta t$ may be calculated by a simple algebraic expression applied to each grid point $(i, j)$. However, the stability analysis of Case I, Appendix D shows no clearly defined region of stability but only one which may be stable for very small time increments. This result is insufficient for two reasons. First, although the initial solution may prove stable for small times, instability may occur at any moment, and if it does, valuable computer time will have been lost. Second, to obtain a stable solution, the time increment may have to be prohibitively small. This will necessitate large machine times to obtain a solution at a physically significant real time.

Two additional explicit formulations of Equation (47) are investigated for stability in Appendix $D$ and actually derived in Appendix E. 
Again, the results are inconclusive. A final finite difference analog to Equation (47) is investigated in which all terms of Equation (47) except the partial derivative of vorticity with respect to time are evaluated at the time $\left(n+\frac{1}{2}\right) \delta t$. A time averaging technique is used to evaluate these terms, and the resulting finite difference equation is implicit. The unknown vorticity $\omega^{n+l}(i, j)$ is expressed in terms of the unknown vorticity at the time $(n+1) \delta t$ at the surrounding grid points. The stability analysis of Case IV, Appendix D indicates that a region of stability exists for this analog. Although it seems to exist only for small Reynolds numbers (an undesirable limitation), there is no stability limit on the time increment. In addition, investigation of the results of Case IV, Appendix D indicates that the small Reynolds number Iimitation is due to the simplification of the VTE by the continuity equation. The difficulty arises specifically from the $\operatorname{term} \frac{v_{r}}{r}$ in the continuity equation. Hence, in the two-dimensional case, the simplification to the VTE which is possible through the employment of the continuity equation will not lead to a small Reynolds number limitation on the stability of the finite difference equation.

The finite difference equation which is used to calculate the vorticity $\omega^{n+1}(i, j)$ in this research is derived from the unsimplified form of the VTE, Equation (31). Equation (31) may be rewritten so that only the partial derivative of vorticity with respect to time remains to the left of the equal sign. The time derivative is approximated by the forward time difference 


$$
\frac{\partial \omega}{\partial t}=\frac{\omega^{n+1}(i, j)-\omega^{n}(i, j)}{\delta t}
$$

and the finite difference approximation to the right-hand side of the transposed equation is evaluated at the time $\left(n+\frac{1}{2}\right) \delta t$ for greater accuracy. The time average technique is applied whereby, for example,

$$
\omega^{n+1 / 2}(i, j)=\frac{1}{2}\left\{\omega^{n+1}(i, j)+\omega^{n}(i, j)\right\}
$$

As shown in Case V, Appendix E, this analog to Equation (3I) is seen to take the form

$$
\begin{aligned}
& \omega^{n+1}(i, j)=\left\{\frac{1}{\delta t}+\frac{1}{\operatorname{Rey}}\left[a^{2}(M-i)^{2}+b^{2}(M-j)^{2}+\frac{1}{2 x^{2}}\right]\right\}^{-1} x \\
& \left\langle\left\{\frac{1}{\delta t}-\frac{1}{\operatorname{Rey}}\left[a^{2}(M-i)^{2}+b^{2}(M-j)^{2}+\frac{1}{2 x^{2}}\right] \omega^{n}(i, j)\right.\right. \\
& +\left\{\frac{a(M-i) v_{z}^{n+l / 2}(i-1, j)}{2}+\frac{a^{2}(M-i)}{R e y}\left[(M-i)+\frac{1}{2}\right]\right\} \times \frac{1}{2}\left[\omega^{n+l}(i-1, j)+\omega^{n}(i-1, j)\right] \\
& +\left\{-\frac{a(M-i) v_{z}^{n+1 / 2}(i+1, j)}{2}+\frac{a^{2}(M-i)}{R e y}\left[(M-i)-\frac{1}{2}\right]\right\} \times \frac{1}{2}\left[\omega^{n+1}(i+1, j)+\omega^{n}(i+1, j)\right] \\
& +\left\{\frac{b(M-j) v_{r}^{n+1 / 2}(i, j-1)}{2}+\frac{b^{2}(M-j)}{\operatorname{Rey}}\left[(M-j)+\frac{1}{2}\left(1-\frac{1}{b x}\right)\right]\right\} x \\
& \frac{1}{2}\left[\omega^{n+1}(i, j-1)+\omega^{n}(i, j-1)\right]
\end{aligned}
$$




$$
\begin{gathered}
+\left\{-\frac{b(M-j) v_{r}^{n+1 / 2}(i, j+1)}{2}+\frac{b^{2}(M-j)}{R e y}\left[(M-j)-\frac{1}{2}\left(1-\frac{1}{b x}\right)\right]\right\} x \\
\left.\frac{1}{2}\left[\omega^{n+1}(i, j+1)+\omega^{n}(i, j+1)\right]\right\rangle
\end{gathered}
$$

where

$$
\begin{aligned}
& v_{z}^{n+1 / 2}(i-1, j)=\frac{b(M-j)}{2 x}\left[\psi^{n+1 / 2}(i-1, j+1)-\psi^{n+1 / 2}(i-1, j-1)\right] \\
& v_{z}^{n+1 / 2}(i+1, j)=\frac{b(M-j)}{2 x}\left[\psi^{n+1 / 2}(i+1, j+1)-\psi^{n+1 / 2}(i+1, j-1)\right] \\
& v_{r}^{n+1 / 2}(i, j-1)=-\frac{a(M-i)}{2 x}\left[\psi^{n+1 / 2}(i+1, j-1)-\psi^{n+1 / 2}(i-1, j-1)\right] \\
& v_{r}^{n+1 / 2}(i, j+1)=-\frac{a(M-i)}{2 x}\left[\psi^{n+1 / 2}(i+1, j+1)-\psi^{n+1 / 2}(i-1, j+1)\right]
\end{aligned}
$$

and, for example,

$$
\psi^{n+I / 2}(i-1, j+1)=\frac{1}{2}\left\{\psi^{n+1}(i-1, j+1)+\psi^{n}(i-1, j+I)\right\}
$$

Equation (48) is an implicit difference equation. The unknown vorticity $\omega^{n+1}(i, j)$ is expressed in terms of the vorticity and the stream function at the surrounding grid points at the time $(n+1) \delta t$. The existence of the unknown stream function in the VTE couples numerically the solutions of the VTE and the DVE; a stream function and vorticity must be found which simultaneously satisfy the two equations. Conse- 
quently, an iterative solution of the two equations is necessary. The stability analysis of Case V, Appendix D, shows that the Reynolds number presents no limitation on the stability of Equation (48). No stability problem exists since the stability criterion may be expressed as

$$
\frac{\text { Rey }}{\delta t}>0
$$

However, an additional point should be mentioned here. Although the stability analysis indicates that the time increment need only be positive, an approximation has already been made which limits the time increment. The time averaging technique states that within the finite difference approximation,

$$
(\delta t) \frac{\partial^{2} \omega}{\partial t^{2}}=\left\{\frac{\omega^{n+1}(i, j)+\omega^{n}(i, j)-2 \omega^{n+1 / 2}(i, j)}{(\delta t)}\right\}=0
$$

Therefore, the time increment must be small enough so that the term $(\delta t) \frac{\partial^{2} \omega}{\partial t^{2}}$ is small.

Thus far, this chapter and the accompanying stability analysis of Appendix D have attempted to show a logical method of attack whereby one may derive the simplest finite difference equation for which there exists an acceptable stability criterion. Initial and Boundary Conditions for the VTE

The initial condition for the VTE, Equation (48), is that at the time $t=0$ 


$$
\omega^{0}(i, j)=\psi^{0}(i, j)=v_{z}^{0}(i, j)=v_{r}^{0}(i, j)=0
$$

for all grid points $(i, j)$ of the mesh. The boundary condition at the center line is unchanged, and

$$
\omega^{n+1}(i, 0)=0
$$

The boundary conditions for large values of $z$ and $r$ are specified at the points $\xi=1$ and $n=1$, respectively. Hence,

$$
\begin{aligned}
& \omega^{n+1}(i, M)=0 \\
& \omega^{n+1}(M, j)=0
\end{aligned}
$$

for all $\mathrm{n}$ or for all time.

At this point, the boundary condition at the solid boundary and in the exit plane must be established. Remember that the boundary value of vorticity in the exit plane has been written as

$$
\omega=a \frac{\partial v_{r}}{\partial \xi}-b(1-n) \frac{\partial v_{z}}{\partial \eta}
$$

The second term of this equation will be known since the axial velocity in the exit plane will have a specified distribution. If this distribution in the $z, r$ plane is of the form 


$$
v_{z}(t, 0, r)=f(r) g(t)
$$

then the boundary value of vorticity may be written as

$$
\omega(t, 0, n)=a \frac{\partial v_{r}}{\partial \xi}-b(1-n) g(t)\left(\frac{\partial f(r)}{\partial r}\right)\left(\frac{\partial r}{\partial \eta}\right)
$$

Since

$$
v_{r}=-\frac{a(l-\xi)}{x} \frac{\partial \psi}{\partial \xi}
$$

combining Equations (55) and (56) and noting that $\xi=0$ on this boundary yields

$$
\omega(t, 0, n)=-\frac{a^{2}}{x}\left\{\frac{\partial^{2} \psi}{\partial \xi^{2}}-\frac{\partial \psi}{\partial \xi}\right\}-b(1-n) g(t)\left(\frac{\partial f(r)}{\partial r}\right)\left(\frac{\partial r}{\partial n}\right)
$$

The problem now is to evaluate the first and second derivatives of $\psi$ with respect to $\xi$ on the boundary $\xi=0$. Having specified that the radial velocity in the exit plane is zero and knowing that it vanishes on the solid boundary reveals that

$$
\left.\frac{\partial \psi}{\partial \xi}\right|_{\xi=0}=0
$$

as seen from Equation (56). From Appendix C, the central difference expression and the accompanying truncation error for this vanishing first derivative is given by 


$$
\frac{\partial \psi(0, n)}{\partial \xi}=\frac{\psi(1, j)-\psi(-1, j)}{2 h}-\frac{h^{2}}{6} \frac{\partial^{3} \psi\left(\xi_{I}, n\right)}{\partial \xi^{3}}=0
$$

where $h$ is the mesh spacing and $\xi_{1}$ lies in the interval $(-h, h)$. This requires that

$$
\psi(-I, j)=\psi(I, j)-\frac{h^{3}}{3} \frac{\partial^{3}\left(\xi_{1}, n\right)}{\partial \xi^{3}}
$$

The central difference and its accompanying truncation error for the second derivative may be written as

$$
\frac{\partial^{2} \psi(0, n)}{\partial \xi^{2}}=\frac{\psi(1, j)+\psi(-1, j)-2 \psi(0, j)}{h^{2}}-\frac{h^{2}}{12} \frac{\partial^{4} \psi\left(\xi_{2}, n\right)}{\partial \xi^{4}}
$$

where $\xi_{2}$ lies in the interval $(-h, h)$. Combining this equation and the previous one gives an expression for the second derivative in terms of mesh points on the boundary and in the interion of the flow field, i.e.,

$$
\frac{\partial^{2} \psi(0, n)}{\partial \xi^{2}}=2 \frac{\psi(1, j)-\psi(0, j)}{h^{2}}-\frac{h^{3}}{3} \frac{\partial^{3} \psi\left(\xi_{1}, n\right)}{\partial \xi^{3}}-\frac{h^{2}}{12} \frac{\partial^{4} \psi\left(\xi_{2}, n\right)}{\partial \xi^{4}}
$$

Unfortunately, this approximation has a truncation error whose magnitude is greater than the others found in this analysis. It is now realized that the boundary formulation proposed by Esch [22] (introduced in Appendix B) gives a truncation error which is consistent with the other derivations of this numerical model. Nevertheless, the proof of the validity of a numerical method lies in the results it produces. It will 
be shown in the convergence study that the interior flow field is relatively insensitive to the errors in the vorticity produced on the boundary. This conclusion will also be substantiated by the solution of a steady state problem.

Combining Equations (57), (58), and (60) and neglecting the truncation error of Equation (60) produces the final boundary condition necessary for the VTE, namely

$$
\begin{aligned}
\omega^{n+1}(0, j) & =-\frac{2 a^{2}}{x}\left\{\frac{\psi^{n+1}(1, j)-\psi^{n+1}(0, j)}{(1 / M)^{2}}\right\} \\
& -b(1-n) g(t)\left(\frac{\partial f(r)}{\partial r}\right)\left(\frac{\partial r}{\partial n}\right)
\end{aligned}
$$

where the functions $g(t)$ and $f(r)$ are yet to be specified. The Finite Difference Analog to the DVE

Once the vorticity distribution has been computed at the new time $(n+1) \delta t$ for all the mesh points $(i, j)$ from the VTE, the corresponding stream function distribution may be determined from the DVE. Applying central differences to Equation (32) and simplifying as outlined in Appendix $\mathrm{F}$ allows derivation of an expression for the stream function at the point $(i, j)$ and the time $(n+I) \delta t$ as given by

$$
\begin{array}{r}
\psi^{n+1}(i, j)=\left\langlea ^ { 2 } ( M - i ) \left\{[(M-i)-1 / 2] \psi^{n+1}(i+1, j)+\right.\right. \\
\left.[(M-i)+1 / 2] \psi^{n+1}(i-1, j)\right\}
\end{array}
$$




$$
\begin{gathered}
+b^{2}(M-j)\left\{[(M-j)-1 / 2(1+I / b x)] \psi^{n+1}(i, j+1)+\right. \\
\left.[(M-j)+1 / 2(1+1 / b x)] \psi^{n+1}(i, j-1)\right\} \\
\left.+x \omega^{n+1}(i, j)\right\rangle\left\{2 a^{2}(M-i)^{2}+2 b^{2}(M-j)^{2}\right\}^{-1}
\end{gathered}
$$

The application of Equation (62) to each interior mesh point of the grid produces a system of $(M-1)^{2}$ simultaneous equations in the unknowns $\psi^{n+1}(i, j)$. This result is similar to the system of simultaneous equations evolving from the implicit formulation of the VTE. Boundary Conditions for the DVE

The DVE, now represented by Equation (62) is elliptic and requires boundary conditions at all times for its solution. In the exit plane and on the solid boundary

$$
\psi(0, j)=\int_{0}^{n} \frac{v_{z}(0, n) x}{b(1-n)} d n
$$

where

$$
n=\frac{j}{M}
$$

and the function $\mathrm{v}_{\mathrm{z}}$ is yet to be specified. Along the center line,

$$
\psi(i, 0)=0
$$

On the remaining boundaries for either $\xi$ near $I$ or $n$ near 1 the 
boundary conditions for the differential equation are given respectively by either

$$
\text { for } \xi \text { near } 1 \quad \frac{\partial \psi}{\partial \xi}=0
$$

or

$$
\text { for } n \text { near } 1 \quad \frac{\partial \psi}{\partial n}=0
$$

Consider first the effect of Equation (45) when it is applied at a point which occurs at any finite radial station $n$ and at any finite axial station $\xi$ between the grid points $i=M-1$ and $i=M$. The stream function at the points $\xi_{1}=\frac{M-1}{M}$ and $\xi_{2}=\frac{M}{M}=1$ may be expressed by Taylor series expansions about the point, $\xi, n$. Thus

$$
\begin{aligned}
& \psi\left(\xi_{1}, n\right)=\psi(\xi, n)-\frac{h_{1}}{1 !} \frac{\partial \psi(\xi, n)}{\partial \xi}+\frac{h_{1}^{2}}{2 !} \frac{\partial^{2} \psi\left(\bar{\xi}_{1}, n\right)}{\partial \xi^{2}} \\
& \psi\left(\xi_{2}, n\right)=\psi(\xi, n)+\frac{h_{2}}{1 !} \frac{\partial \psi(\xi, n)}{\partial \xi}+\frac{h_{2}^{2}}{2 !} \frac{\partial^{2} \psi\left(\bar{\xi}_{2}, n\right)}{\partial \xi^{2}}
\end{aligned}
$$

where $h_{1}$ and $h_{2}$ are the appropriate mesh spacings and $\bar{\xi}_{1}$ and $\bar{\xi}_{2}$ are the appropriate mean values of the transformed axial distance. Combining these two expressions with Equation (45) specifies the Neumann condition

$$
\psi(M, j)=\psi(M-1, j)
$$

Similarly, for $n$ near 1 , 


$$
\psi(i, M)=\psi(i, M-I)
$$

The truncation error of both these approximations is no greater than $h^{2}$ which is the truncation error of the DVE, Equation (62). Equations (63), (64), (65), and (66) completely specify the boundary conditions needed for the solution of the DVE. However, to simplify the computations, it is shown in Appendix $F$ how equations (65) and (66) may be incorporated into the DVE.

The governing equations and their initial and boundary conditions are summarized in the following table which lists the appropriate equation numbers.

Table 1. Summary of Equations, Initial Conditions, and General Boundary Conditions

\begin{tabular}{lcc}
\hline $\begin{array}{l}\text { Governing } \\
\text { Equation }\end{array}$ & $\begin{array}{c}\text { Initial } \\
\text { Conditions }\end{array}$ & $\begin{array}{c}\text { Boundary } \\
\text { Conditions }\end{array}$ \\
\hline VTE - (48) & $(49)$ & $(50),(51),(52),(61)$ \\
DVE - (62) & & $(63),(64),(65),(66)$ \\
\hline
\end{tabular}

The boundary values of vorticity and the stream function have been indicated in Equations (61) and (63) in functional notation. At this point, the axial velocity distribution in the exit plane which is given by the expression 


$$
v_{z}(0, r, t)=f(r) g(t)
$$

is to be specified.

\section{The Specific Problems Investigated}

The two problems studied in detail in this investigation differ from each other only in the specified form of the axial velocity distribution in the exit plane. The first will be called the parabolic case, and the second the uniform case. Although the latter is not strictly uniform, it is nearly so; the name is more or less a mnemonic device.

The form of Equation (67) may now be developed. The dimensionless axial velocity was introduced as

$$
v_{z}=\frac{v^{*}}{U}
$$

In the exit plane this may be rewritten as

$$
v_{z}=\frac{v_{z}^{*}}{U_{C L}} \frac{U_{C L}}{U}
$$

where $U_{C L}$ is the dimensional center line velocity at any time. The first term on the right-hand side is the ratio of the velocity at a particular radial position to the velocity at the center line at any particular time, and it is referred to as the velocity profile. Hence

$$
\frac{\mathrm{v}_{\mathrm{Z}}^{*}}{\mathrm{U}_{\mathrm{CL}}}=f(r) \quad \text { alone }
$$


The second term, the ratio of the center line velocity to the reference velocity, is referred to as the center line profile and may be stated as

$$
\frac{\mathrm{U}_{C L}}{\mathrm{U}}=g(t) \text { alone }
$$

For both cases studied, the center line profile $g(t)$ is specified to be that of two linear segments as shown in Figure 5. The finite instantaneous inpulse of Figure 6 was not employed for two reasons. First, it is felt that the finite instantaneous impulse would tax the numerical solution because initially the changes in the flow field would be large and convergence of iterative techniques within the model would prove difficult. This difficulty was experienced by Payne [17] in his numerical solution of the two-dimensional viscous starting jet. Second, it is felt that of the two profiles shown, the impulse of a linear acceleration is more nearly that which would be found in an experimental investigation.

Of the two velocity profiles $f(r)$ specified, the one parabolic and the other uniform, the latter is probably more realistic. However, it is expected that these two profiles will bracket or be the limiting cases of the actual circumstances likely to occur. The Parabolic Profile

The parabolic profile is the familiar one found in the solution of the Navier-Stokes equations for laminar flow through a round tube. The dimensional velocity distribution is given by 


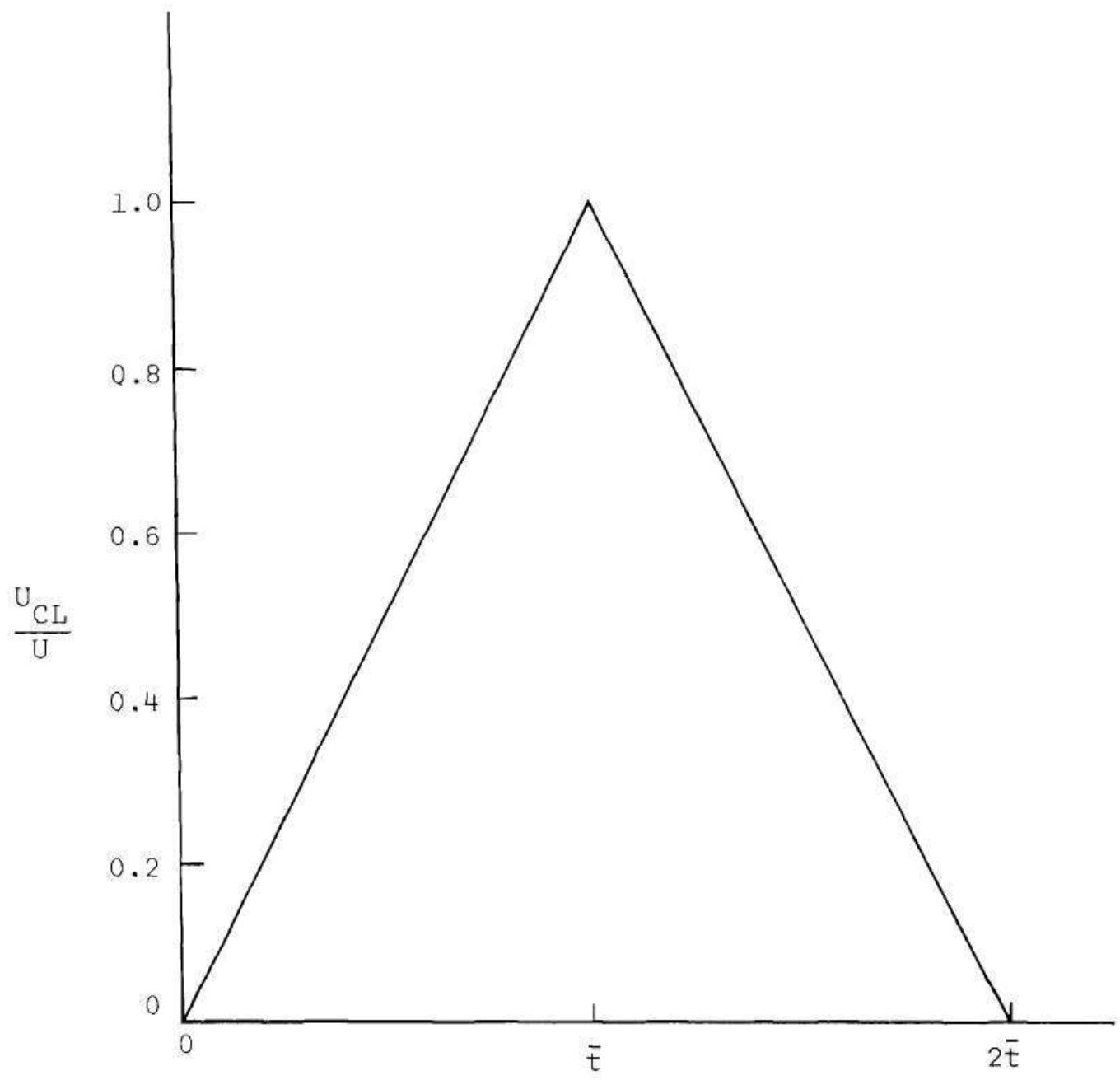

Figure 5. Linear Impulsive Center Line Profile 


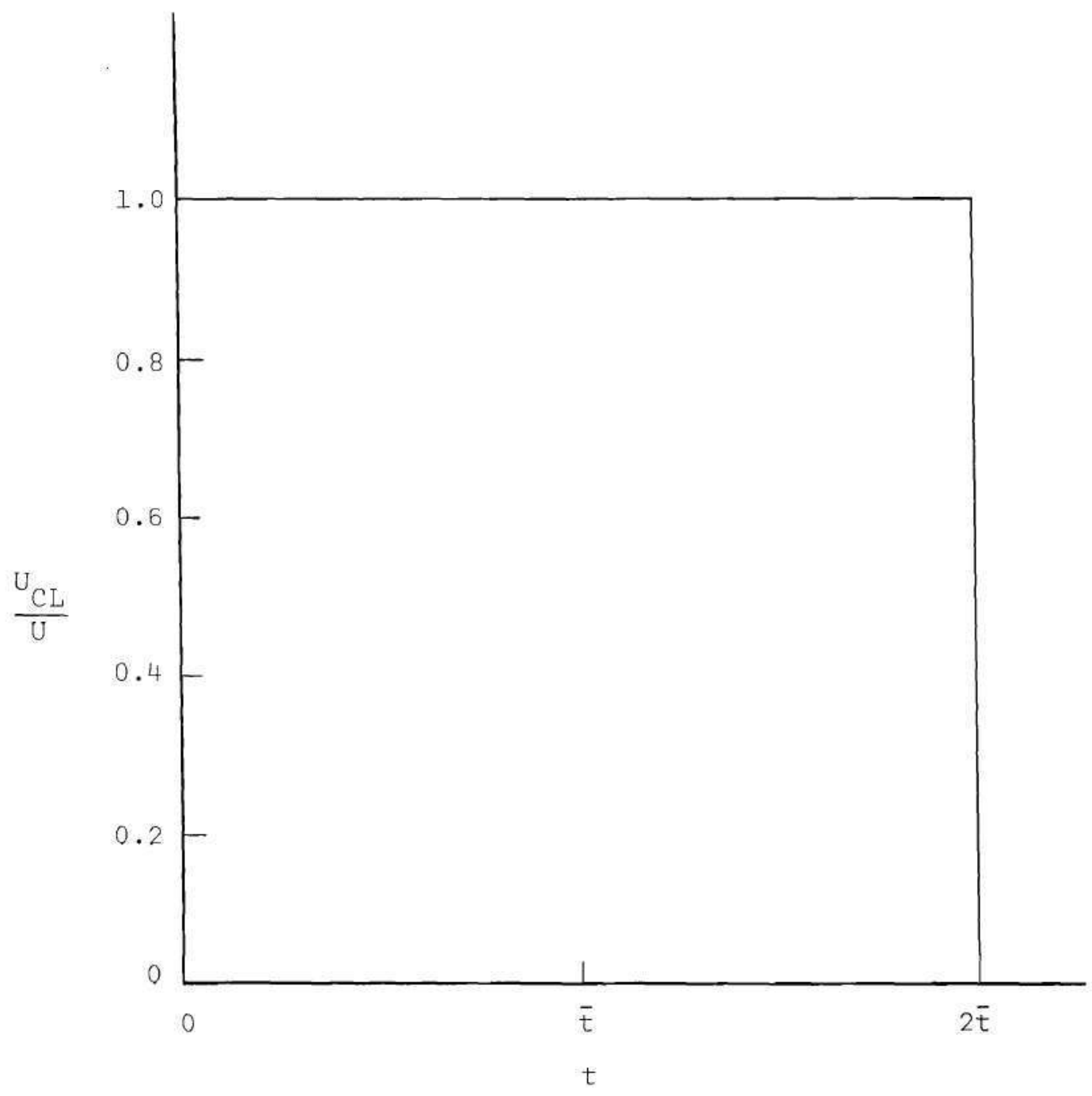

Figure 6. Instantaneous Impulsive Center Line Profile 


$$
v_{z}^{*}\left(r^{*}\right)=-\frac{1}{4 \mu^{*}} \frac{d p^{*}}{d z^{*}}\left(R^{2}-r^{* 2}\right)
$$

where $R$ is the radius of the tube. Dividing through by the center line velocity, $v_{z}^{*}\left(r^{*}=0\right)$, gives the dimensionless velocity distribution

$$
\begin{aligned}
& \frac{\mathrm{v}_{\mathrm{z}}^{*}(r)}{\mathrm{U}_{\mathrm{CL}}}=\left(1-r^{2}\right) \\
& \text { for } 0 \leq r \leq 1
\end{aligned}
$$

Equation (68) now allows evaluation of the boundary value of vorticity in Equation (61) and the boundary value of the stream function in Equation (63). The boundary condition on the vorticity in the transformed plane may be written at $\xi=0$ as

$$
\omega^{n+1}(0, j)=-\frac{a^{2}}{x} 2\left\{\frac{\psi^{n+1}(1, j)-\psi^{n+1}(0, j)}{(1 / M)^{2}}\right\}+2 g(t) x
$$

for $0 \leq x \leq 1$. Naturally, for $x>1$, i.e., on the solid boundary, the velocity must vanish, so the last term goes to zero. This may all be incorporated into the one equation

$$
\omega^{n+1}(0, j)=-\frac{a^{2}}{x} 2\left\{\frac{\psi^{n+1}(1, j)-\psi^{n+1}(0, j)}{(1 / M)^{2}}\right\}+\sigma_{P}\{2 g(t) x\}
$$

where 


$$
\sigma_{P}= \begin{cases}1 & \text { for } j \leq P \\ 0 & \text { for } j>P\end{cases}
$$

where $j=P$ is the mesh point corresponding to the tube radius $r=x=1$, where

$$
x=r=\frac{1}{b} \ln \left(\frac{M}{M-j}\right)
$$

and where the function $g(t)$ is given in Figure 5. In a similar manner, the boundary value of the stream function, Equation (63), may be simplified to the form

$$
\psi^{n+1}(0, j)=\left\{\sigma_{P} \frac{x^{2}}{2}\left(1-\frac{x^{2}}{2}\right)+\frac{\left(1-\sigma_{P}\right)}{4}\right\} g(t)
$$

where $\sigma_{p}, x$, and $g(t)$ take on the same values as in Equation (69). The Uniform Profile

The uniform velocity profile is shown in Figure 7 . Mathematically, the axial component of velocity in the exit plane may be stated as 

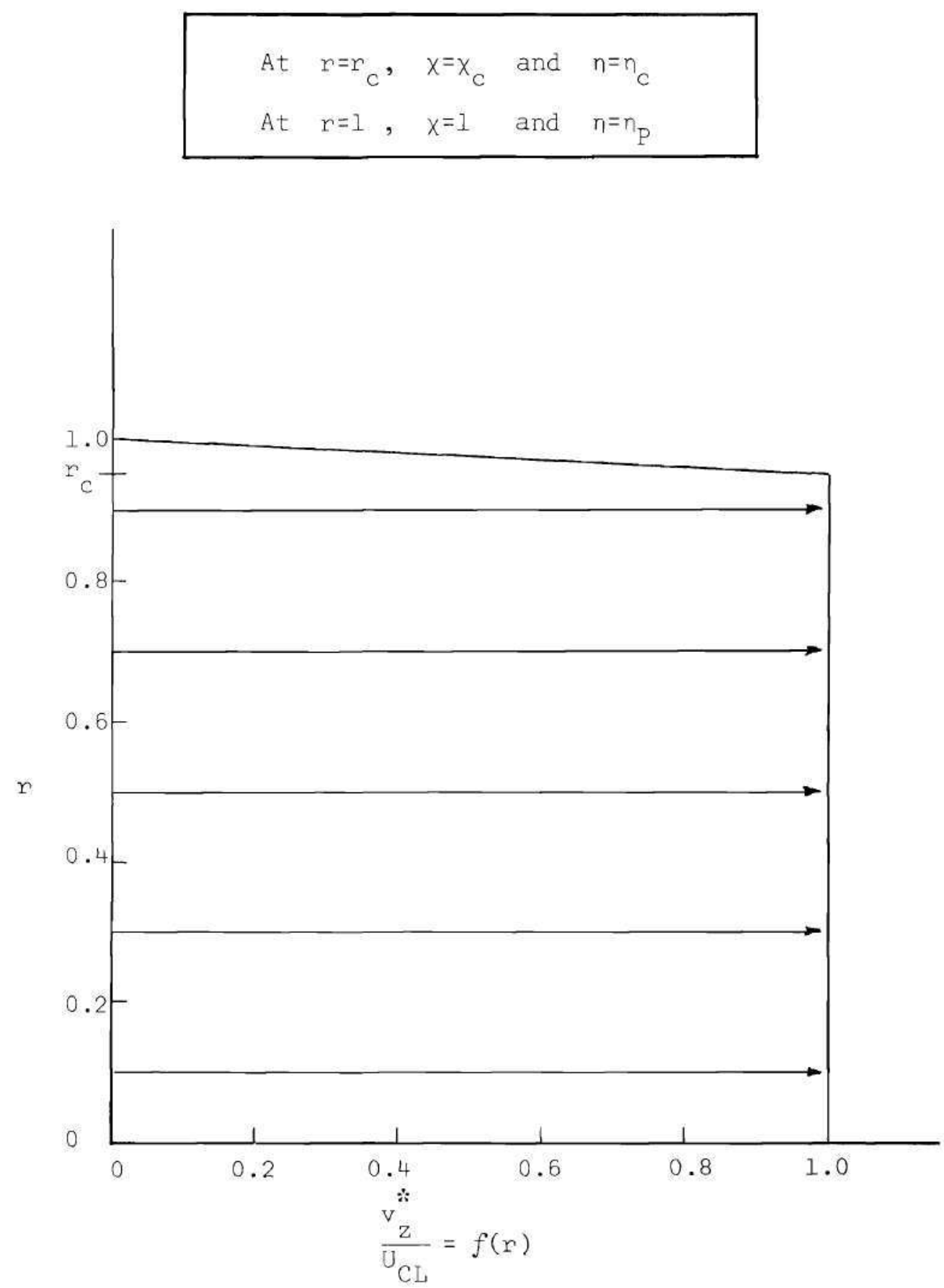

Figure 7. Uniform Velocity Profile 


$$
\left.\begin{array}{lll}
v_{z}=g(t) & \text { for } & 0 \leq \leq r_{c} \\
v_{z}=\frac{1-r}{1-r_{c}} g(t) & \text { for } & r_{c}<r \leq 1 \\
v_{z}=0 & \text { for } & r>1
\end{array}\right\}
$$

The parameter $r_{c}$ is the radial station of the exit plane at which the discontinuity of the derivative of velocity occurs in Figure 7 . After carrying out the differentiation implied in Equation (61) and simplifying, the boundary value of vorticity may be expressed by

$$
\omega^{n+1}(0, j)=-\frac{2 a^{2}}{x}\left\{\frac{\psi^{n+1}(1, j)-\psi^{n+1}(0, j)}{\left(\frac{1}{M}\right)^{2}}\right\}+\frac{\sigma_{u} g(t)}{1-x_{c}}
$$

where

$$
\begin{gathered}
x_{c}=r_{c} \\
\sigma_{u}=\left\{\begin{array}{lll}
1 & \text { for } & j=P \\
0 & \text { for } & j \neq P
\end{array}\right.
\end{gathered}
$$

and $g(t)$ is given by Figure 5 . The parameter $x_{c}$ is given a convenient value so that only the mesh point at $i=0$ and $j=P$ experiences any vorticity due to the radial derivative of the axial velocity in the exit plane. Now the difficulty can be seen that stems from a strictly uni- 
form profile for which $\chi_{c}$ has the value of unity. The contribution to the boundary vorticity of the term involving the radial derivative of the axial velocity, the last term of Equation (72), vanishes everywhere in the exit plane except at the generating tube radius, $x=1$, at which point it becomes infinite. To avoid this difficulty, the profile was altered as shown in Figure 7 to allow both the satisfaction of the noslip condition and the existence of a finite vorticity at the wall of the vortex generating tube. The boundary value of the stream function is found by transforming Equation (71) to the $\xi, n$ plane, substituting the result into Equation (63), performing the integration, and simplifying. The results of these operations give

$$
\begin{gathered}
\psi^{\mathrm{n}+1}(0, j)=\sigma_{u} \frac{1}{2} g(t) x^{2}+\left(\sigma_{u}-1\right)\left\langle\frac{g(t)}{1-x_{c}}\left\{x^{2}\left[\frac{1}{2}-\frac{x}{3}\right]\right\}\right. \\
\left.\left.-x_{c}^{2}\left[\frac{1}{2}-\frac{x_{c}}{3}\right]\right\}\right\rangle
\end{gathered}
$$

where $\sigma_{u}, g(t), x$, and $x_{c}$ have the same meaning as in Equation (72). Now the governing equations, their initial conditions, and their boundary conditions for two particular cases, the parabolic velocity profile and the uniform velocity profile, have been derived in terms of a few simple constants. In an attempt to clarify the following discussion of the computational procedure, the pertinent equations are listed by their equation numbers in the following table. 
Table 2. Summary of Equations, Initial Conditions, and Specific Boundary Conditions

\begin{tabular}{lccc}
\hline $\begin{array}{l}\text { Governing } \\
\text { Equation }\end{array}$ & $\begin{array}{c}\text { Initial } \\
\text { Conditions }\end{array}$ & $\begin{array}{c}\text { Conditions } \\
\text { Parabolic Profile }\end{array}$ & $\begin{array}{c}\text { Boundary } \\
\text { Conditions } \\
\text { Uniform Profile }\end{array}$ \\
\hline VTE - (48) & $(49)$ & $(50),(51),(52),(69)$ & $(50),(51),(52),(72)$ \\
DVE - (62) & & $(64),(65),(66),(70)$ & $(64),(65),(66),(73)$
\end{tabular}

The constants necessary to completely specify the two problems which are about to be solved are chosen to correspond to a physically realistic case. The vortex is generated in ain from a hole of radius 0.04 feet by a velocity impulse (shown in Figure 5) which reaches a maximum value of two feet per second. These values give a Reynolds number of

$$
\text { Rey }=533
$$

Figure 5 shows the impulsive velocity to act over a dimensionless period of $2 \bar{t}$. The dimensional period of the impulse is taken to be 4/10 of a second, and consequently

$$
\bar{t}=10.0
$$

After some experimentation, the time increment for the calculations was 
taken to be

$$
\delta t=0.5
$$

(It is interesting to compare this time increment for the implicit technique employed here to that of Kurzrock and Mates [35], $\delta t \cong 10^{-6}$, for the explicit numerical solution of a compressible problem in air.) Finally, the parameter $x_{c}$ of Figure 7 is set at

$$
x_{c}=0.95
$$

so that the component of vorticity produced by the gradient of the axial velocity in the exit plane is felt at the grid point $i=0$ and $j=P$. This point is located at the edge of the exit plane. For all cases $P$ takes the value

$$
P=\frac{M}{2}
$$

so that in the radial direction half the grid points fall within $r=1$ and the other half fall outside this region. Inspection of Equation (26) shows that the transformation constant may be calculated as

$$
b=\ln \left(\frac{M}{M-P}\right)=\ln (2)
$$

Initially the transformation constant a is set equal to b. After the 
generated vortex has moved far enough into the field to affect the vorticity at the grid points near $i=M$, the constant $a$ is decreased and $a$ new grid is generated. Any point in the new mesh for which $i=M-1$ is located at a greater axial distance $z$ from the exit plane than is the corresponding point in the old grid. Values of the dependent variables in the new mesh are computed from the ones in the old mesh by linear interpolation.

The governing equations have now been derived in finite difference form in the transformed plane. The equations and the techniques employed in their solution were programmed in ALGOL for solution on the Burrough's B-5500, a high-speed digital computer.

\section{The Computational Procedure}

It is assumed in the following discussion that the properties of the flow field, i.e., the vorticity, stream function, and velocity distributions, are known at the time (n) $\delta t$. Clearly, for the case $\mathrm{n}=0$ the values of these dependent variables vanish as evidenced by Equation (49). The procedure to be presented will then yield distributions of the dependent variables at the time $(n+1) \delta t$.

The procedure is as follows:

1. A first guess at the vorticity distribution at the time $(n+1) \delta t$ is made by employing an explicit formulation of the VTE such as Equation (E-5) of Appendix E.

2. The boundary conditions on the stream function at $t=(n+1) \delta t$ are calculated, using either Equations (64), (65), (66), and (70) for the parabolic profile or Equations (64), (65), (66), and 
(73) for the uniform profile.

3. The stream function distribution corresponding to the first guess of the vorticity distribution at $t=(n+1) \delta t$ is calculated by iterating the system of equations which arise from the application of the DVE, Equation (62), at each interior mesh point. The most expedient iterative technique proved to be that of Method $V$ of Appendix G. This is a method of successive over-relaxation which has been speeded up slightly. Also, it may be noted that the iteration may be simplified somewhat by incorporating the boundary conditions expressed by Equations (65) and (66) into the DVE. This is explained in Appendix F.

4. The boundary condition on vorticity, given by Equation (69) for the parabolic profile or Equation (72) for the uniform profile, is evaluated at the time $(n+1) \delta t$ from the stream function distribution. 5. An improved guess at the new vorticity distribution may be computed by the VTE, Equation (48), as a function of the vorticity and stream function distributions at the time $t=(n) \delta t$ and as a function of the previous guesses at the new distributions at the time $t=(n+1) \delta t$. The system of simultaneous equations arising from the application of Equation (48) is solved by the technique discussed as Method VI in Appendix G. This iterative procedure, the alternating direction method, is the only one which converged when the non-linear terms of Equation (48) were large.

$$
\text { 6. A stream function distribution at }(n+1) \delta t \text { is calculated }
$$
corresponding to the latest guess of the vorticity found in Step 5 . This is done in the manner of Step 3. The corresponding boundary value of vorticity is also calculated as in Step 4. 
7. Steps 5 and 6 may now be repeated until successive guesses at the new vorticity distribution at the time $(n+1) \delta t$ correspond within some predetermined tolerance of the iteration. The tolerance used throughout this study is 1 per cent. When successive iterates at the same point in space agree within I per cent, the iterative procedure is terminated.

8. A final stream function distribution corresponding to the vorticity computed in Step 7 is determined by the method of Step 3 .

In order to explain this computation procedure further, a flow chart is given in Figure 8.

Once the vorticity, velocity, and stream function distributions are computed in the transformed plane, they may be expressed in the $\mathrm{z}, \mathrm{r}$ plane by the inverse transformations,

$$
\begin{aligned}
& z=\frac{1}{a} \ln \left(\frac{1}{1-\xi}\right) \\
& r=\frac{1}{b} \ln \left(\frac{1}{1-n}\right)
\end{aligned}
$$

\section{Verification of the Numerical Model}

At this point the numerical model has been derived with careful consideration given to its stability and accuracy, and a computational procedure has been developed which gives a solution to the numerical model. The next step of the analysis is to investigate these results and to demonstrate that they are reasonable. This verification is conducted in two separate studies. In the first, a standard convergence 


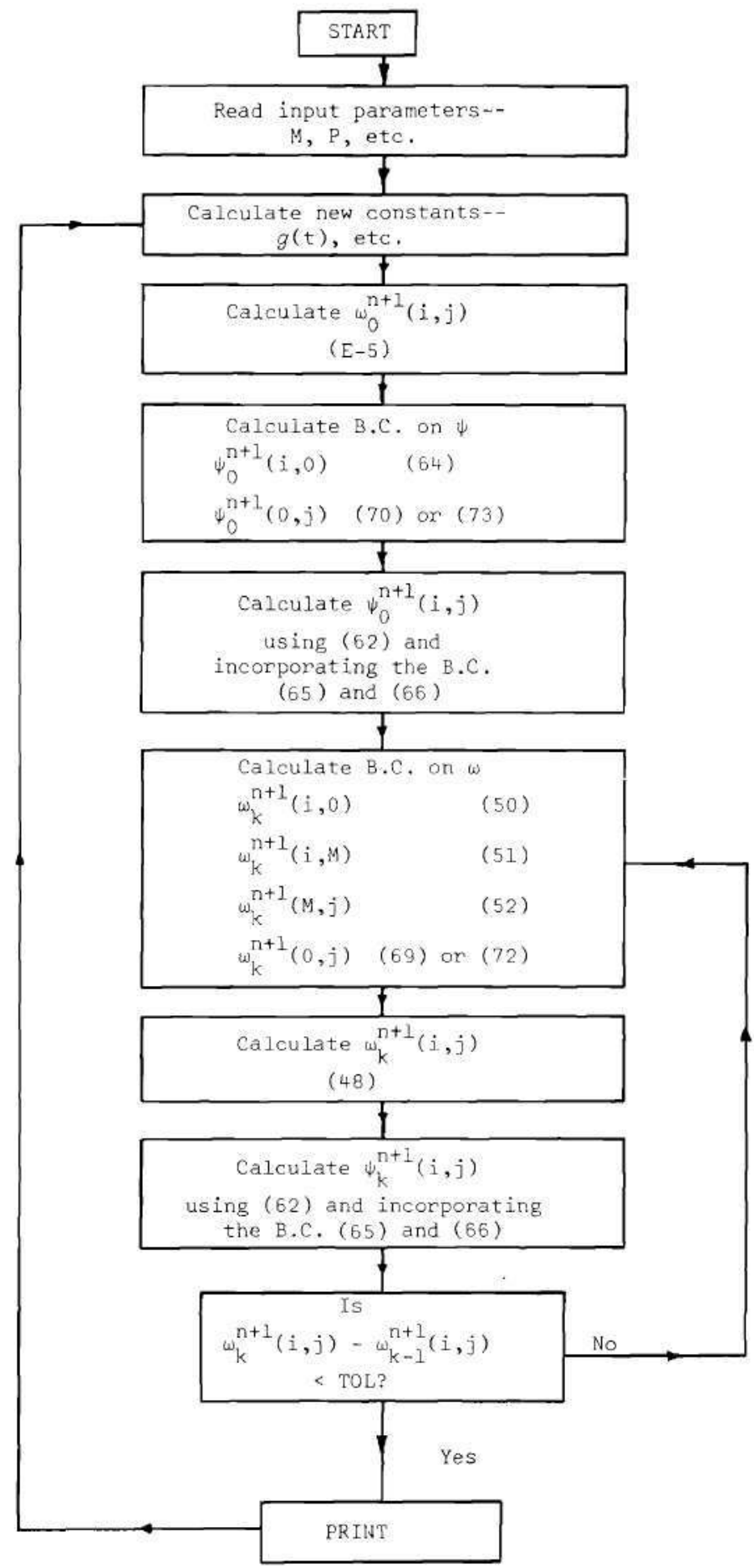

NOTE: The subscript denotes the approximation number of a given distribution.

Figure 8. Schematic Diagram of the Computational Procedure 
analysis is carried out and presented in detail in Appendix $H$. In the second, a steady state solution of the finite difference equations is found. This analysis is given in Appendix I. A short discussion of each of these investigations is given in the following paragraphs.

Consider first the convergence study of Appendix $\mathrm{H}$. Each of the two cases examined, the parabolic profile and the uniform profile, were solved for two different mesh sizes in the transformed plane. For the parabolic profile, the time step increments were also different, so the results showed simultaneously the consequence of varying size in both the spatial grid and the time step. This approach had a disadvantage in that the difference between the solutions could not be traced to either the grid spacing or the time step individually. For the uniform profile, the solutions were found for both grids isochronally so the variation in results could be ascertained as a consequence of the spatial mesh only.

Two cases, $\delta t=1.0, M=16$ and $\delta t=0.5, M=24$, are considered for the parabolic profile. The vorticity distributions (the vorticity contours or isovorts) and the stream function distributions (the streamlines) over the $z, r$ plane at various times show good qualitative agreement. The vorticity and axial velocity appear to agree within about 10 per cent, the radial velocities compare within a few per cent, and the difference between the values of the stream function cannot be distinguished. A comparison of the boundary values of vorticity as a function of time shows good qualitative agreement. It is noted that a large difference in vorticity on the boundary does not produce a large 
difference of vorticity in the interior of the flow field.

Two cases, $\delta t=0.5, M=16$ and $\delta t=0.5, M=24$, are considered for the uniform profile. The vorticity distributions and the streamline patterns show good qualitative agreement over a portion of the flow field. However, in the case of the uniform profile, a new phenomenon occurs which involves the decay of very small regions of negative vorticity in the part of the $z, r$ plane which is downstream of the orifice. The grid is evidently not fine enough here to allow the same degree of convergence for the uniform case as for the parabolic case.

A quantitative comparison is made of the dependent variables as a function of time at a point in the flow field near where the small regions of negative vorticity are decaying. The vorticities of the two cases sometimes vary from each other by a factor of three. However, both the axial and radial velocities show a maximum difference of about 20 per cent between the two mesh sizes, and the stream function shows a maximum difference of about 10 per cent.

It is apparent from the convergence study that the parabolic profile case gives better agreement for the two mesh sizes studied than does the uniform profile case. Although it is known that the speed of convergence depends on the particular problem investigated, the difference between the rates of convergence of the two problems studied here is an interesting result. Numerically, the two problems differ only in the velocity profile specified in the exit plane. The difference in the rates of convergence may be understood by consideration of the truncation error. As shown in Appendix $C$, the truncation error of a particular partial derivative is the product of a term containing the 
mesh spacing and a neglected derivative which is evaluated by the mean value theorem at some station on the interval over which the finite difference approximation is made. In considering two finite difference approximations made on the same grid, it follows that the term which has the larger neglected derivative will have the greater truncation error. The magnitude of this neglected derivative may be related to those of the lower order derivatives. Of two solutions to the same partial differential equation, the one experiencing the greater changes will effect the larger derivatives and the larger truncation errors. Observation of the figures of Appendix $\mathrm{H}$ reveals that for a given mesh spacing, the changes in the dependent variables of the uniform profile case are greater than those of the parabolic profile case. This leads to a greater truncation error and a poorer agreement in the uniform profile solutions than the parabolic profile solutions for the same change in the grid size.

It is concluded that although the approximation for the uniform profile case may not be so good as desired, convergence of the technique has been demonstrated. The long computer time necessary to solve the uniform profile case for a grid composed of more points prohibits a further investigation.

Consider now the second step of the verification of the numerical model, a comparison of the steady state solution of the numerical model to that of a model simulated by the boundary layer equations. The details of the analysis are given in Appendix I. A solution given in Pai [36] for flow issuing from a round hole of infinitesimal radius had to be tailored to the geometry of Figure 2. The vorticity distribu- 
tion resulting from Pai's solution was input to the numerical model, and the computer calculated the corresponding stream function distribution. Using $M=24$ (i.e., 529 interion mesh points) the numerical solution was found at 12 succeeding time steps $(\delta t=0.5)$, and the difference between both the initial and final vorticity and stream function distributions, respectively, could not be distinguished. This demonstrates that the numerical model preserves the solution of the boundary layer equations for this axisymmetric free jet.

It would have been desirable to solve the numerical model for the axisymmetric free jet over a period extending from time $t=0$ at which all velocities are zero to some $t$ at which a steady state solution is achieved. The extensive computer time necessary to effect such a solution prohibited this procedure. Nevertheless, the approach considered here did serve to verify the method. In addition, another favorable conclusion was reached. The method of calculating the boundary value of vorticity in the exit plane and on the solid boundary appears to be valid since no difficulties arose from this approximation.

The two studies employed to verify the numerical model, the convergence study and the steady state solution, show that the numerical model of this investigation is a valid approximation to the NavierStokes equations for $M=24$. Convergence of the solution has been demonstrated by the favorable comparison of results for two different mesh sizes. The steady state solution indicates that a solution of the boundary layer equations is also a solution of the Navier-Stokes equations for a problem which is accurately described by both systems of equations. 
CHAPTER IV

RESULTS OF THE NUMERICAL MODEL

In this chapter the results of the numerical calculations are presented for the two problems studied, the first being the parabolic profile case and the second being the uniform profile case. All computations were performed on a mesh composed of 529 interior mesh points $(M=24)$ with a time step increment of $\delta t=0.5$. All iterative procedures were terminated when successive iterates at any particular point in time and space agreed with each other within 1 per cent. The results presented for the parabolic profile represent 18 hours of computer time, and the results for the uniform profile represent 10 hours of computer time on the Burrough's B-5500 high-speed digital computer. The length of time necessary to obtain these results prohibited any further numerical study.

The numerical results are shown graphically in a number of ways. The initial plots show streaklines which correspond to the smoke pictures seen by an observer in a laboratory. Next, a typical figure illustrating the velocity vectors in a portion of the field is given. This is to again demonstrate that the numerical solution is consistent with the flow phenomena which one usually associates with a vortex. Next, a series of figures presents lines of constant vorticity and lines of constant stream function. These are given in the $z, r$ plane for discrete times in the history of the vortex. The vorticity contours 
simply show curves possessing the same value of vorticity and rotational velocity experienced by a small volume of the fluid. The stream function contours are really the lines which mark the intersection of instantaneous stream surfaces and the meridian plane. Stream surfaces are used because the flow is three-dimensional, and a particular stream surface is one which satisfies the requirement that the velocities are tangent to it. However, since the azimuthal velocities of this problem are zero due to symmetry, a line of intersection like the one mentioned above does satisfy the definition of a streamline, i.e., a line along which the velocity vectors are tangent. The stream function contours will hereafter be referred to as streamlines. A physical discussion of the ring development accompanies the figures illustrating the lines of constant vorticity and the streamlines. Finally, a number of plots which are representative of the velocities in the field are shown.

\section{The Parabolic Profile}

The Streaklines

A streakline represents the instantaneous locations of a number of fluid particles all of which emanated from the same spatial location at different times. Streaklines are most commonly observed in a smoke tunnel. The smoke pictures of the early development of a vortex ring illustrated in Figure 1 are just the aggregate of a large number of streaklines which have their origins at various radial stations of the exit plane. In the investigation of Margavey and MacLatchey [31] described earlier, a thin column of smoke rising next to the vortex generator was used to investigate a vortex ring produced in the immediate 
vicinity of the smoke; this smoke forms a streakline.

A streakline should not be confused with either a pathline or a streamline. A pathline is the locus of positions of a particular fluid element at succeeding times, whereas a streamline is a line to which the velocity vectors are tangent at a particular time. In steady flow streaklines, pathlines, and streamlines coincide, but in unsteady flow they must be carefully differentiated from each other.

The problem of computing a streakline is necessarily that of calculating the locations of individual particles. Using a Lagrangian description, the position of a fluid element at a particular time is given by the vector equation

$$
\bar{r}\left(z_{0}, r_{0}, t_{0}, t\right)=\int_{t_{0}}^{t} \bar{v}\left(z_{0}, r_{0}, t_{0}, t\right) d t
$$

where $\bar{r}$ and $\bar{V}$ are the position and velocity of a particular element which is identified by its initial position $\left(z_{0}, r_{0}\right)$ at the time it is initially released into the flow field $\left(t_{0}\right)$. In a numerical method, the known parameters are the velocities at discrete points in space and time. Hence, the integral must be replaced by a summation of the form

$$
\bar{r}\left(z_{0}, r_{0}, t_{0}, t\right)=\sum_{i=1}^{N} \bar{v}_{i}\left(z_{0}, r_{0}, t_{0}, t\right) \delta t_{i}
$$

where $i$ is a dummy index and $N$ is the number of time increments such that 


$$
t=\sum_{i=1}^{N} \delta t_{i}
$$

Two sources of error are inherent in this summation for a finite difference method. The first of these is the inaccuracy introduced in obtaining the velocities $\bar{V}_{i}$ by linear interpolation, and the second is the error due to the finite size of the time increment. Of the two errors, the one due to the time increment is the more important because of the accumulation of error. For this reason, the streakline patterns are presented only for the early stages of vortex development.

In this study, fluid elements are introduced into the flow at each time step from a number of radial stations in the exit plane. At a given time the velocity of each particle is computed by linear interpolation of the velocities at the surrounding grid points of the finite difference mesh. The velocity of each element is considered constant over the time increment so that the distance traversed by an element in the time step is the product of the velocity and the time increment. At any time of interest the particle positions may be plotted, and the lines joining the positions of elements emanating from the same station in the exit plane are the streaklines.

The streakline patterns for the parabolic profile case are given in Figures 9 through 13 at the times $t$ equal to 2, 6, 10, 14, and 18 . (Remember that the period of the impulsive velocity input is $2 \bar{t}=20$, so the maximum velocity in the exit plane occurs at $\bar{t}=10$.) The dots appearing on each streakline are the locations of the fluid elements at that time, and the dashed line of each figure denotes the interface 


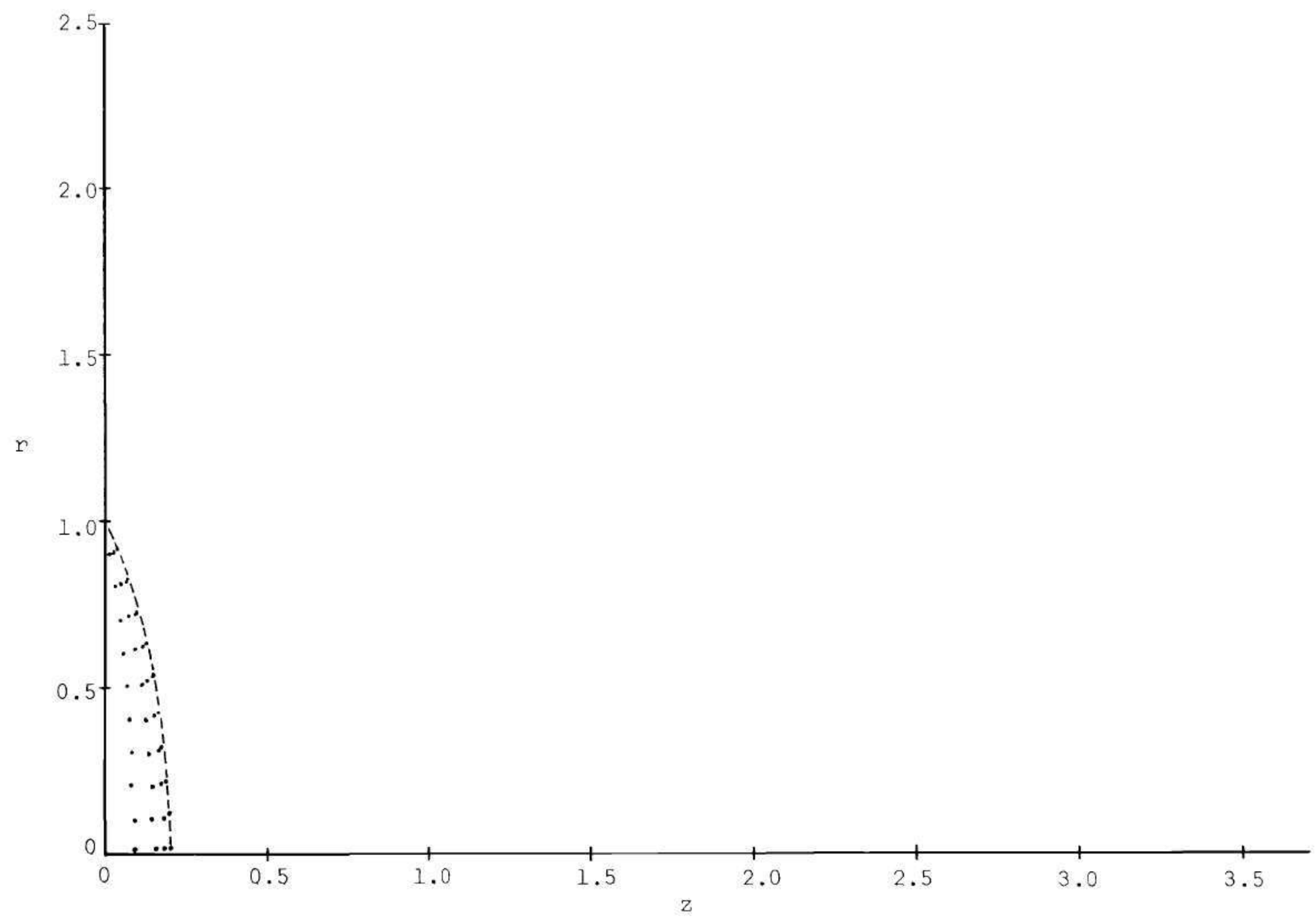

Figure 9. Streakline Pattern at $t=2$-- Parabolic Profile 


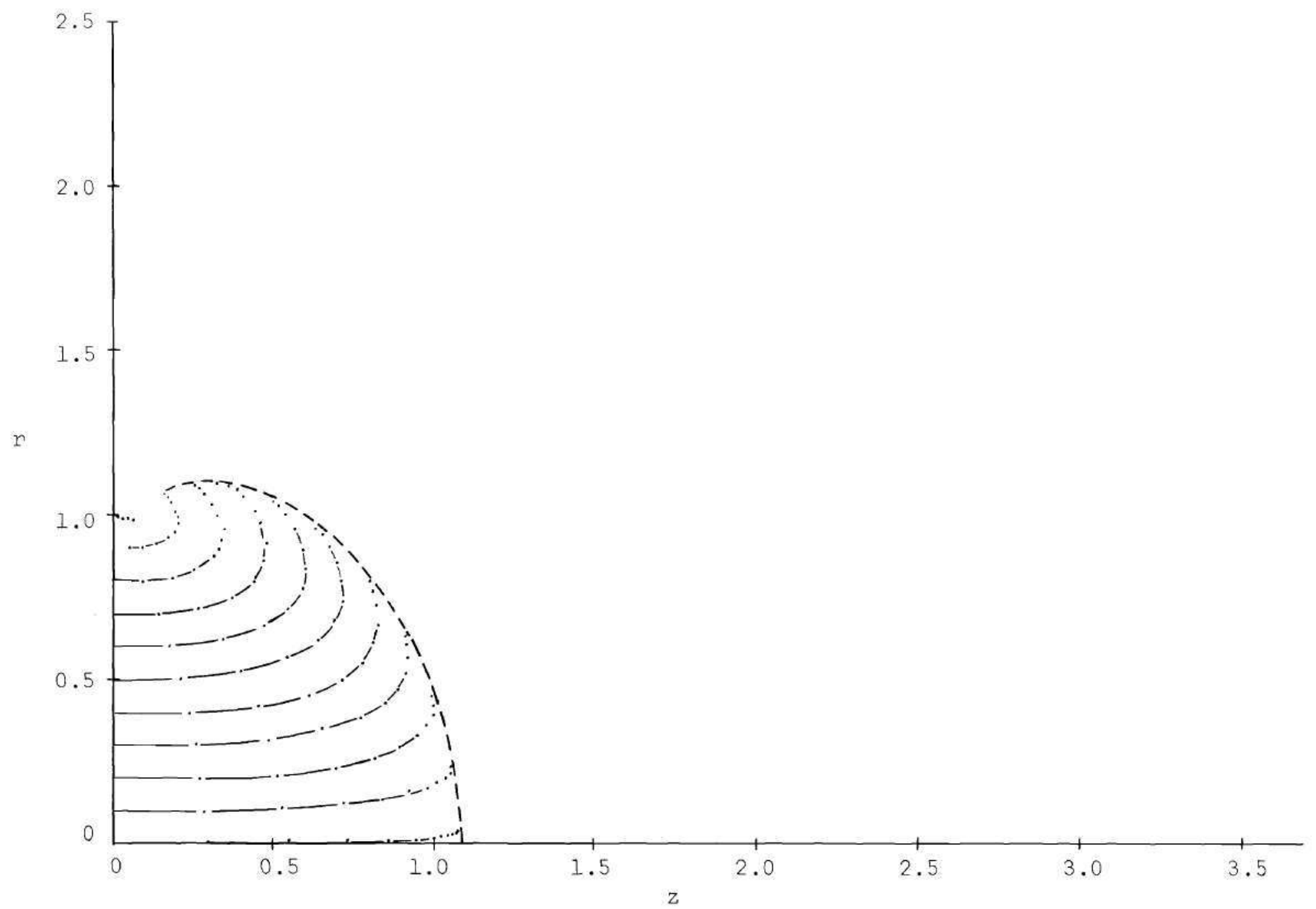

Figure 10. Streakline Pattern at $t=6$-- Parabolic Profile 


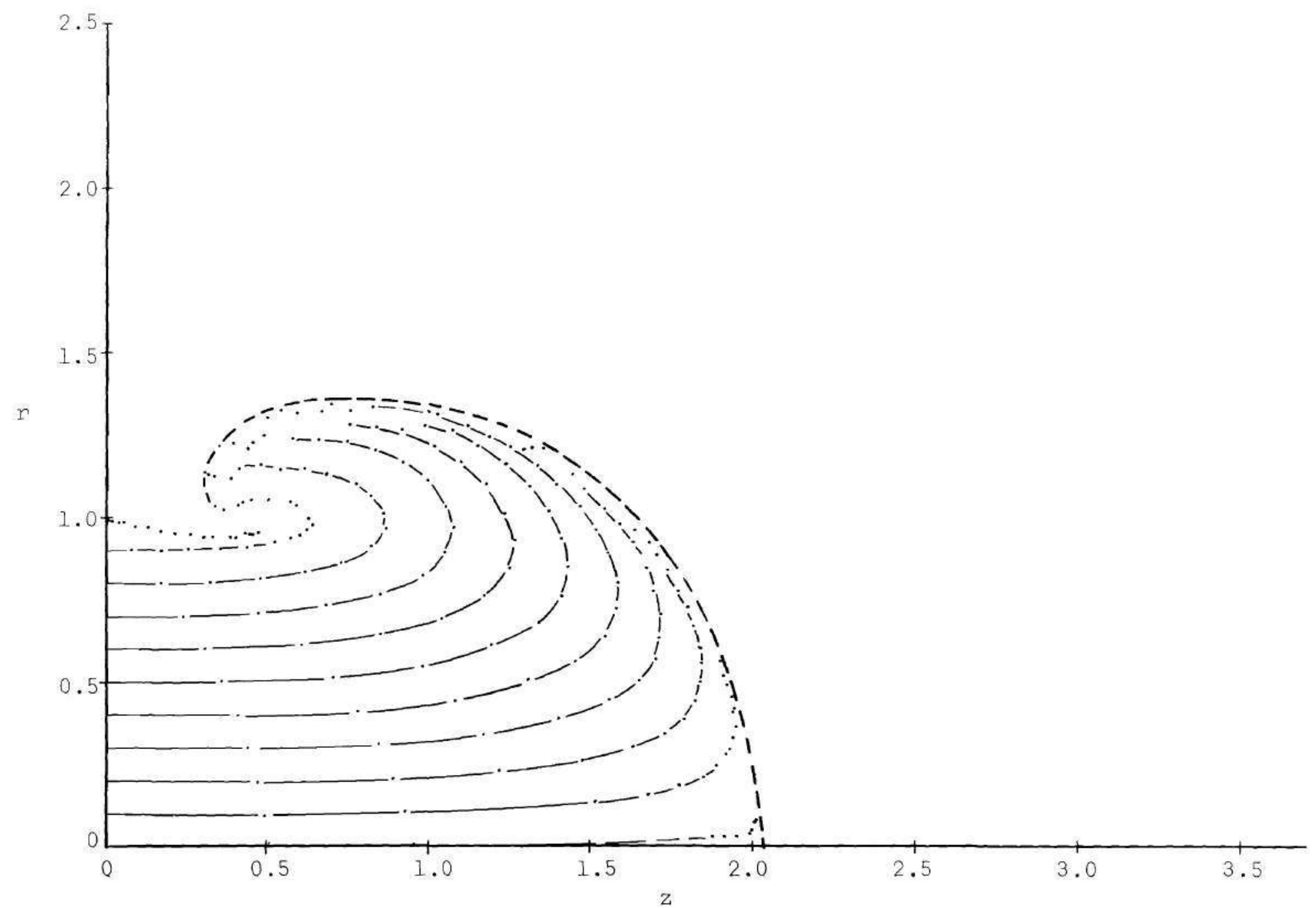

Figure 1l. Streakline Pattern at $t=10$-- Parabolic Profile 


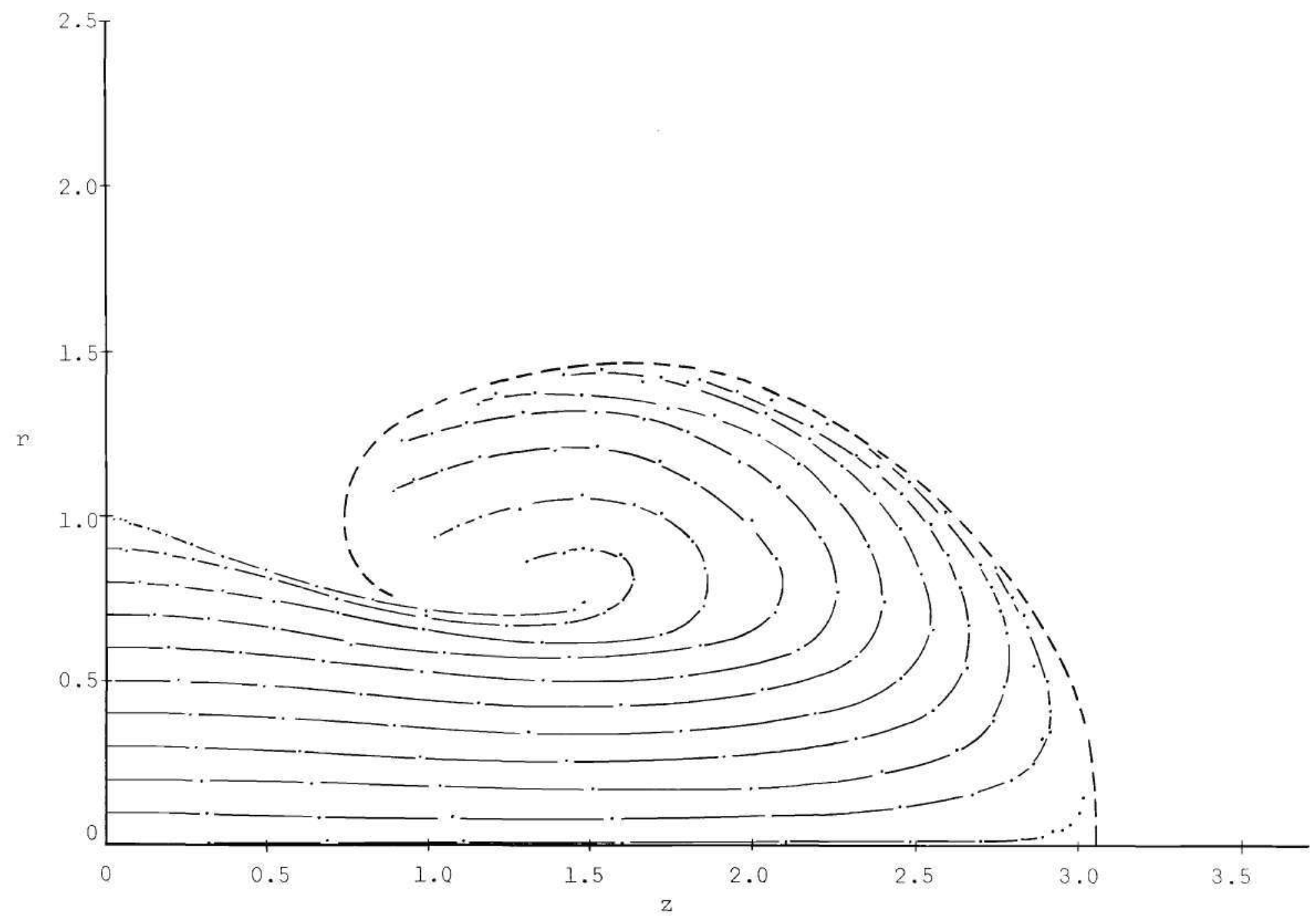

Figure 12. Streakline Pattern at $t=14$-- Parabolic Profile 


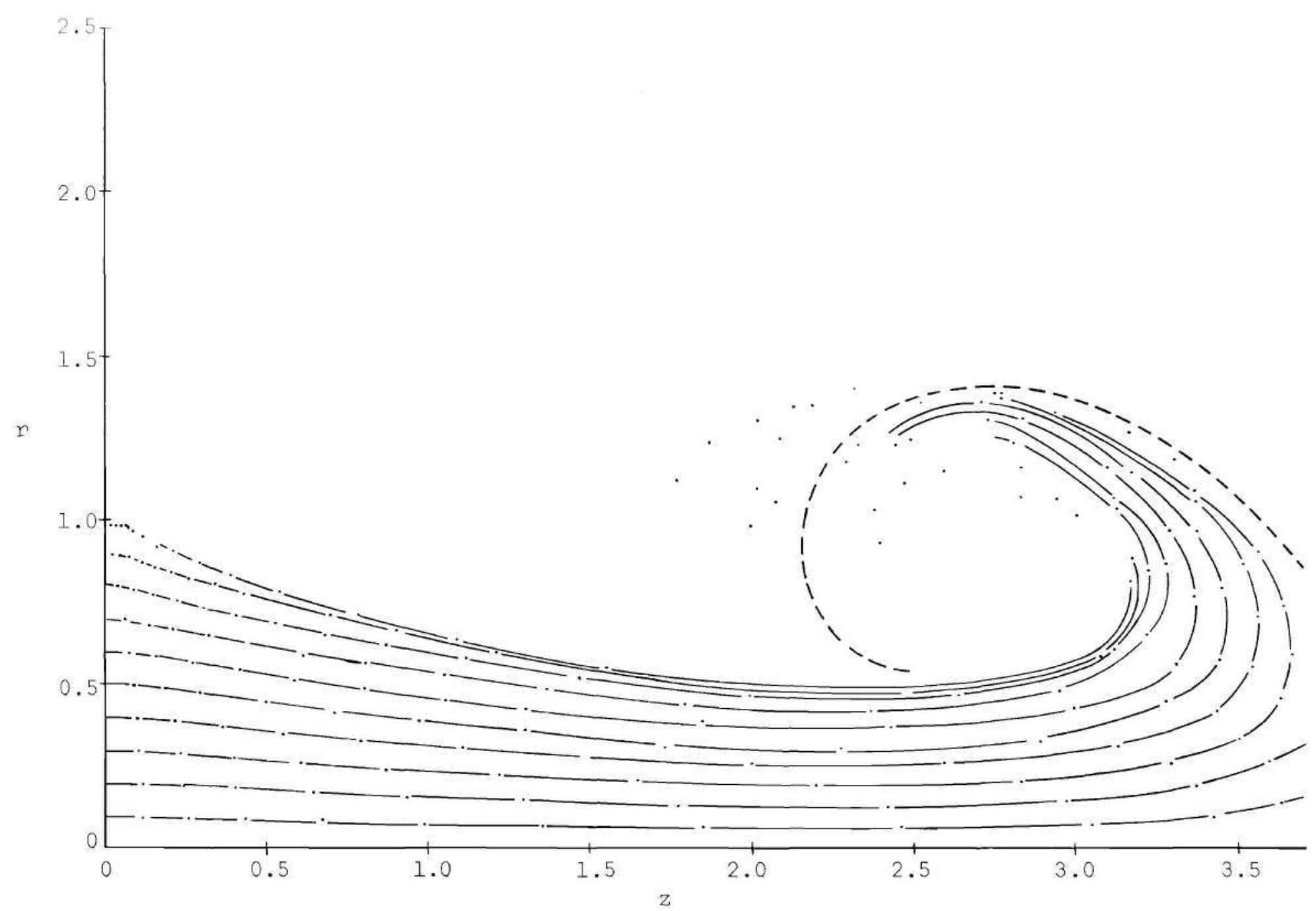

Figure 13. Streakline Pattern at $t=18$-- Parabolic Profile 
between the air ejected from the generator tube and the air in the ambient surroundings. For simplicity and clarity, in much of the following discussion the air from the generator tube will be referred to as smoke. This is the terminology which would be used in the laboratory if smoke were injected into a large volume of air.

Figure 9 shows little more than the fact that the velocity profile of the smoke is indeed parabolic. Since the fluid elements are introduced at equal increments of time, the larger distances between particles near the exit plane indicate that these elements are moving faster than the ones further out in the field. The leading elements of smoke are moving both axially and radially out into the flow field.

Figure 10 shows the interaction between the smoke and the ambient surroundings beginning to take effect as fluid near the wali is being entrained into the jet. The rotational velocities of the elements near the edge of the jet are high. In the same region, the low velocity fluid elements which left the exit plane initially have now moved radially into the flow field. The smoke emitted from the exit plane at a somewhat later time with a greater velocity has now moved further downstream and less in the radial direction than the previously emitted smoke. Near the center of the jet, the motion of the fluid is almost purely translational. The mushroom shape of Figure $I$ is beginning to form.

In Figure 11 the velocity in the exit plane has reached a maximum. Near the edge of the jet, some of the fluid elements have moved downstream more than twice as far as elements which left the same radial station of the exit plane at earlier times. The streakline nearest the edge of the jet is now clearly turning toward the center line under 
the influence of the surrounding air. Somewhere above the edge of the jet a stagnation flow is occurring on the solid wall. Above this region the air is moving away from the jet, and below it the air is moving toward the jet. The existence of this stagnation region on the wall is attested by the numerically computed velocity distribution there. However, this stagnation flow and the effect of the wall are only of secondary importance since rings have been successfully generated from tubes for which the area of the tube end is very small.

Although the velocity in the exit plane has not yet started to decrease, the effect of the fluid which has been entrained into the jet can be clearly seen. The diameter of the column of smoke is diminishing as it moves away from the exit plane. Smoke has almost completely surrounded the apparent center of the vortex, and the hole at the center of the ring to which Okabe and Inoue [30] refer is beginning to form. In addition, the center of the ring is moving in the axial direction quite quickly now. However, the highest velocity fluid is still the smoke being ejected from the exit plane.

In Figure 12, the velocity of the smoke entering the field is now diminishing. The diameter of the smoke column is shrinking quite rapidly behind the vortex ring, and in a moment it will pinch off as illustrated in Figure 1-d. A comparison of Figure 12 and Figure 1 shows that a number of convolutions have appeared in Figure $I$ whereas only about one has appeared at this time in Figure 12. This discrepancy is probably due to a number of reasons. First, the impulse of this numerical study has not been strong enough (i.e., had high enough velocities) nor has the impulse lasted long enough to warrant a swirl which winds upon 
itself several times. Second, due to the numerical error inherent in the computation of the trajectory of a fluid element, it is questionable whether such complex convolutions can be computed in this study.

At this time, the highest velocities belong to fluid elements occupying the region downstream of the exit plane. The smoke which came from the exit plane first has now changed direction again, and it is being drawn toward the jet where its velocity increases due to the momentum transfer from the higher energy smoke in the jet.

Figure 13 shows the vortex ring now well embedded in the flow field. The spiral of smoke is beginning to form, and the minimum diameter of the smoke column at this time between the ring and the boundary has diminished to about half of its original size. The surrounding air is being continually pumped into the jet of smoke, and the center is almost surrounded by smoke. The velocities in the exit plane have almost vanished, and the highest velocities in the field occur between the center of the vortex and the center line of symmetry. It is apparent that the results being produced by the computer are describing the generation of a vortex ring as shown in Figure 1 . The Velocity Vector Field

Figure 14 illustrates a typical velocity vector field over a portion of the $z, r$ plane. The time is $t=10$, and the center line velocity in the exit plane is a maximum, i.e., $v_{z}(0,0)=1$. The most woticeable property of the field is the presence of large velocity gradients across the vortex. Another point of interest is the location of the center of the swirl at $z \cong 0.45$ and $r=1.2$. This point does not coincide with the center indicated by the streakline pattern of Figure 


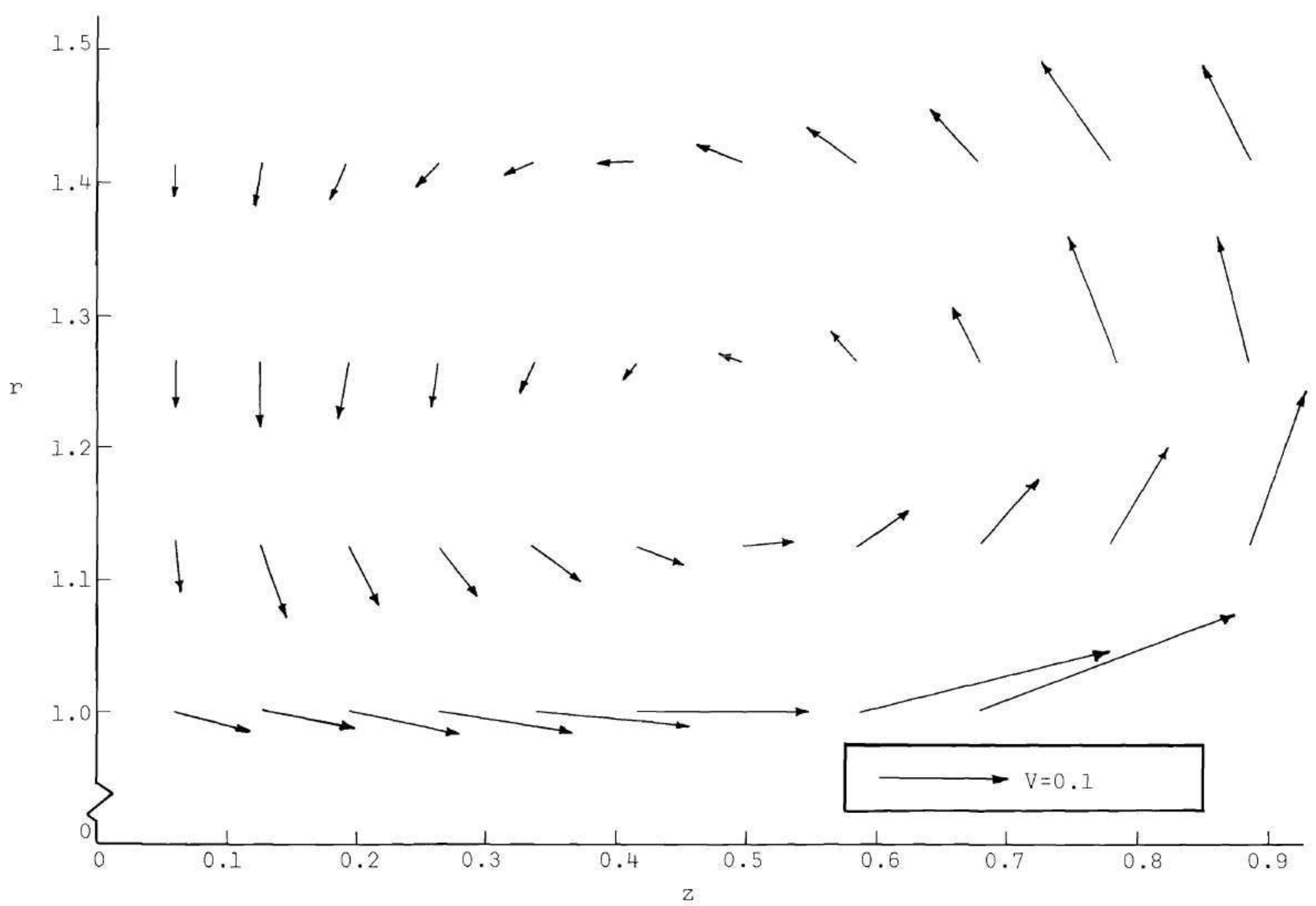

Figure 14. Portion of Velocity Vector Field at $t=10--$ Parabolic Profile 
11. In Figure 11, the center appears to be located at $z \tilde{=} 0.45$ and $r \cong 1.0$. This difference in locations of the vortex center can be explained by the fact that Figure 14 shows the instantaneous velocities of a field which includes the velocity of the vortex itself. The center of a vortex is usually defined as a point of zero velocity in a coordinate system which is moving with the speed of the vortex. Hence, in the fixed co-ordinate system of Eigure 14, the actual center of the vortex occurs at $r \cong 1.1$, and the fluid element at this point is moving in the axial direction with the velocity of the vortex. If the co-ordinate system is changed to one moving with the speed of the ring, the same type of swirl in the flow field will occur, but the point of zero velocity will coincide with the center of the vortex. This change of the co-ordinate system will be introduced later in connection with some further investigations of the flow field of the vortex.

Another phenomenon which may be clearly seen in Figure 14 is that of the fluid being entrained along the wall and into the jet. A velocity gradient normal to the wall may be also observed. The velocity is zero at the wall due to the no-slip condition, and it increases with the distance from the wall. The increasing of velocity continues until the velocity reaches a maximum near $z \cong 0.12$ at which point it begins to decrease as the fluid elements approach the axial location of the vortex center.

The vector plot of Figure 14 is typical, and observations like the ones above may also be made about the vector field at any other time.

Figure 15 presents the instantaneous streamlines at the time $t=10$. Since these streamlines are plotted for equal increments of the 


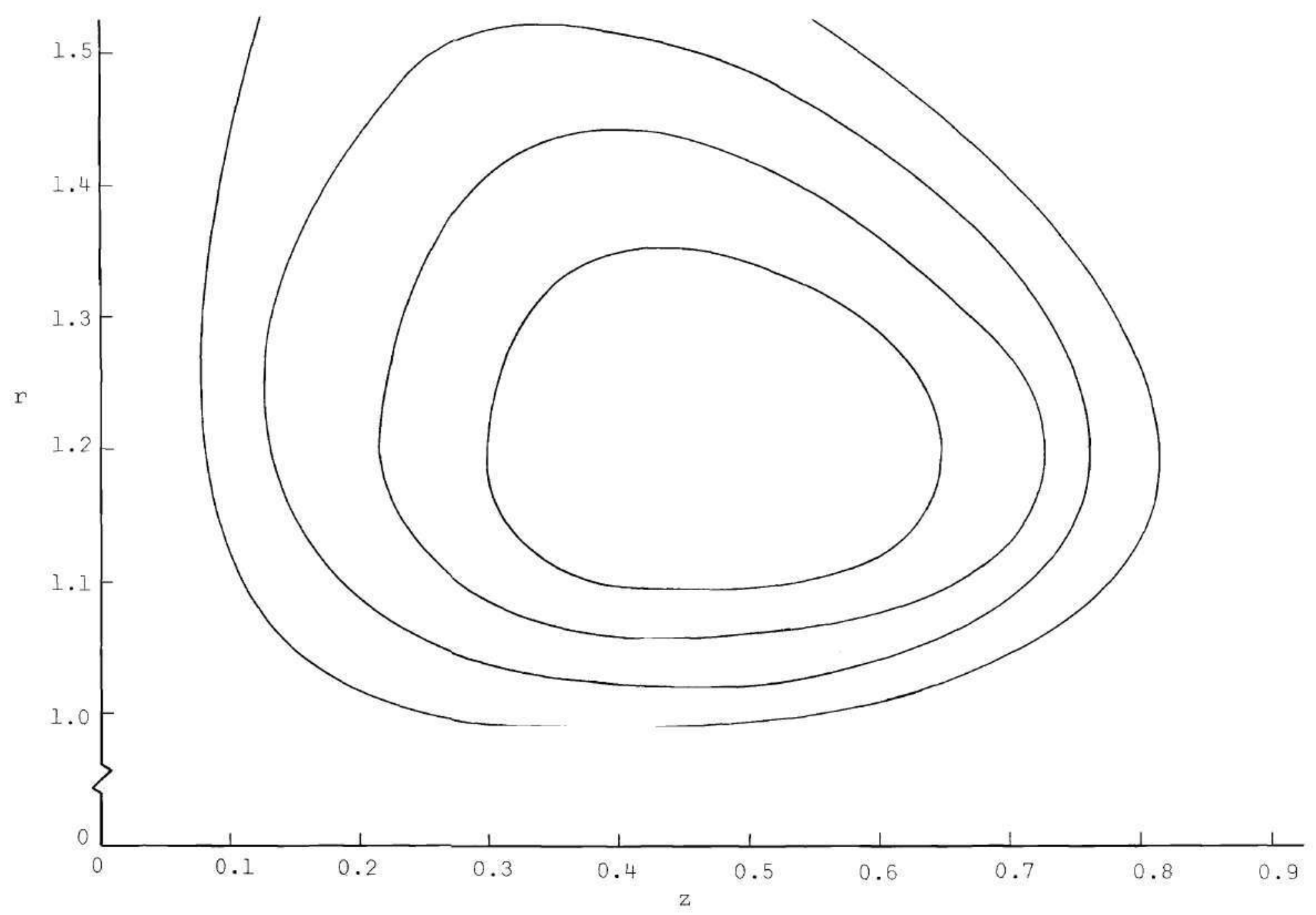

Figure 15. Portion of Streamline Pattern at $t=10_{--}$Parabolic Profile 
stream function, equal increments of the mass flow rate are confined between streamlines. When the streamlines are shown in this manner, the velocity magnitudes are indicated as well as the velocity directions. However, due to the three-dimensionality of the problem, a small divergence of the streamlines for even an intermediate value of the radius reflects a large change in the velocity magnitude. This can be seen by comparing the change in magnitudes of the velocities of Figure 14 with the divergence of the streamlines of Figure 15 for that portion of the field above and to the right of the center of the streamline pattern. Nevertheless, the phenomena which may be observed on a vector plot may also be seen by studying the instantaneous streamline pattern. These phenomena include the movement of the fluid down the wall and into the jet, the velocity gradient normal to the wall, and the existence of high velocity fluid elements in the region around $z \cong 0.7$ and $r \cong 1.0$. In addition, the point of zero velocity in the flow may be easily seen on the streamline plot. Again, it must be remembered that this point does not coincide with the center of the vortex. The Vorticity Distributions and Streamline Patterns

The instantaneous vorticity contours and streamlines are presented in Figure 16 through Figure 29 at the times $t=4,8,12, \cdots, 56$. This period extends from the time the vortex is generated to the time at which the ring has almost achieved a steady state velocity. By taking advantage of the symmetry of the problem, the vorticity distribution and the streamline pattern can be presented in the $z, n$ plane on the same figure at a particular time. The center line of symmetry is the abscissa along which the dimensionless axial distance $\mathrm{z}$ is plotted, and the 


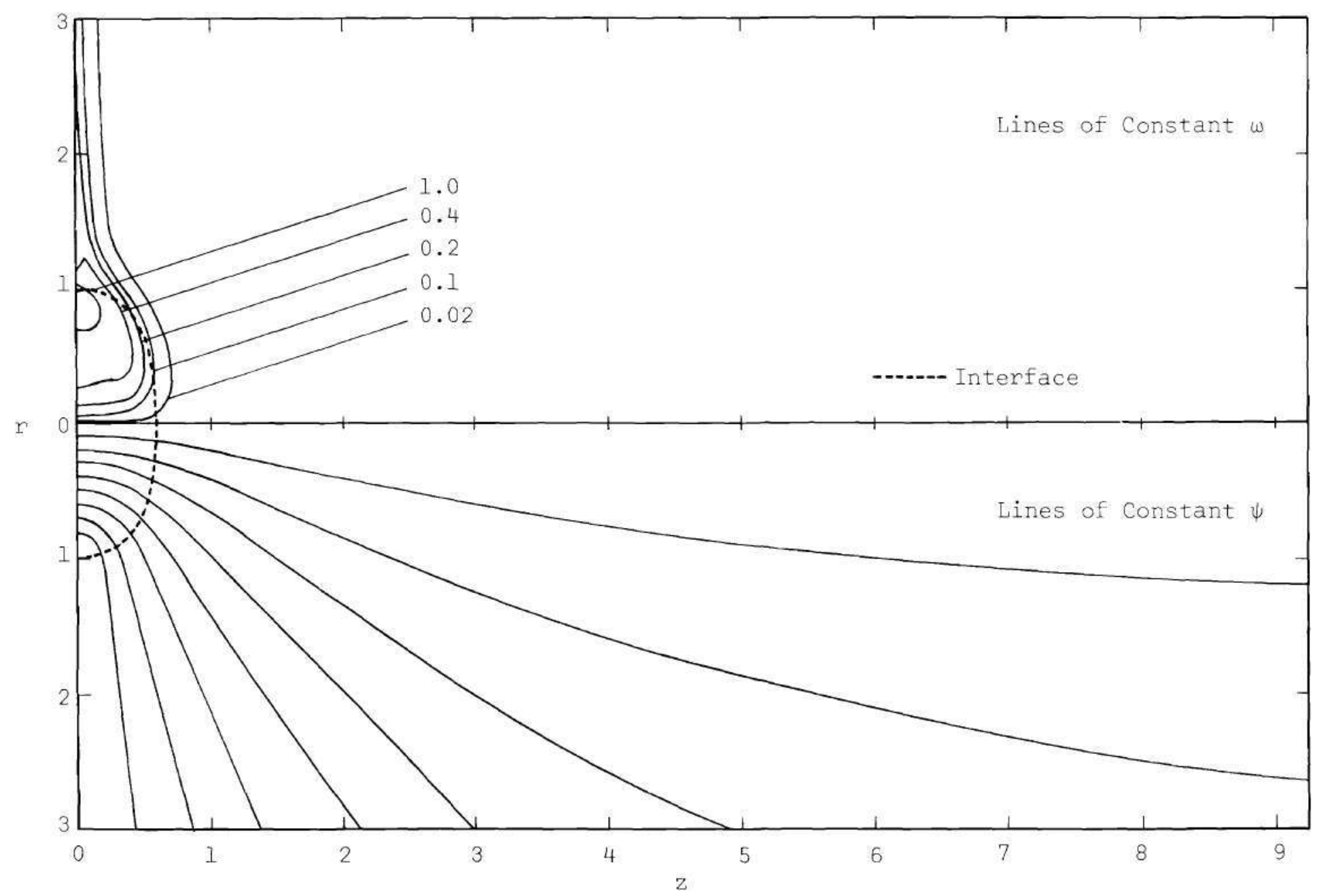

Figure 16. Vorticity Distribution and Streamline Pattern at $t=4$-- Parabolic Profile 


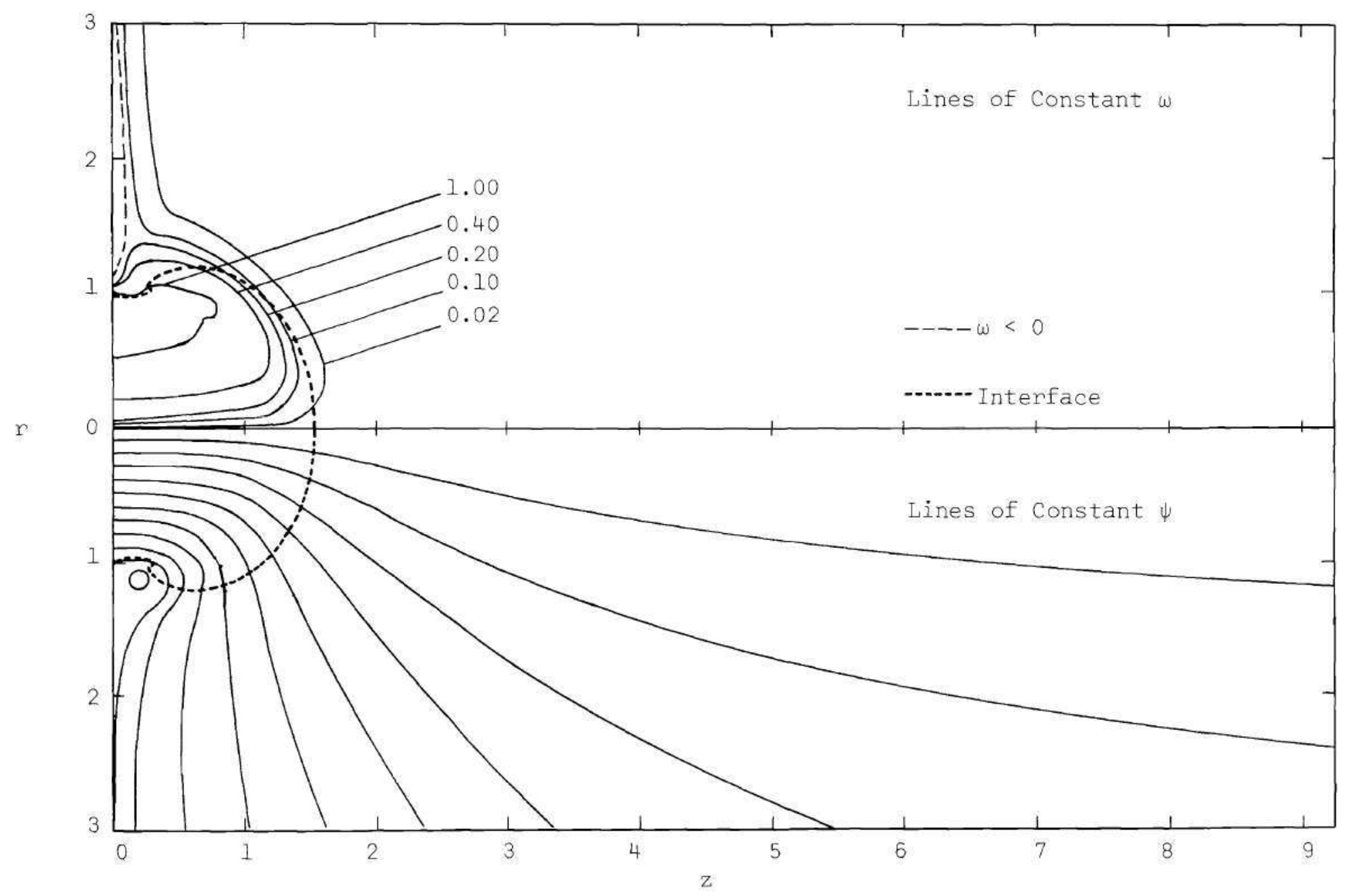

Figure 17. Vorticity Distribution and Streamline Pattern at $t=8--$ Parabolic Profile 


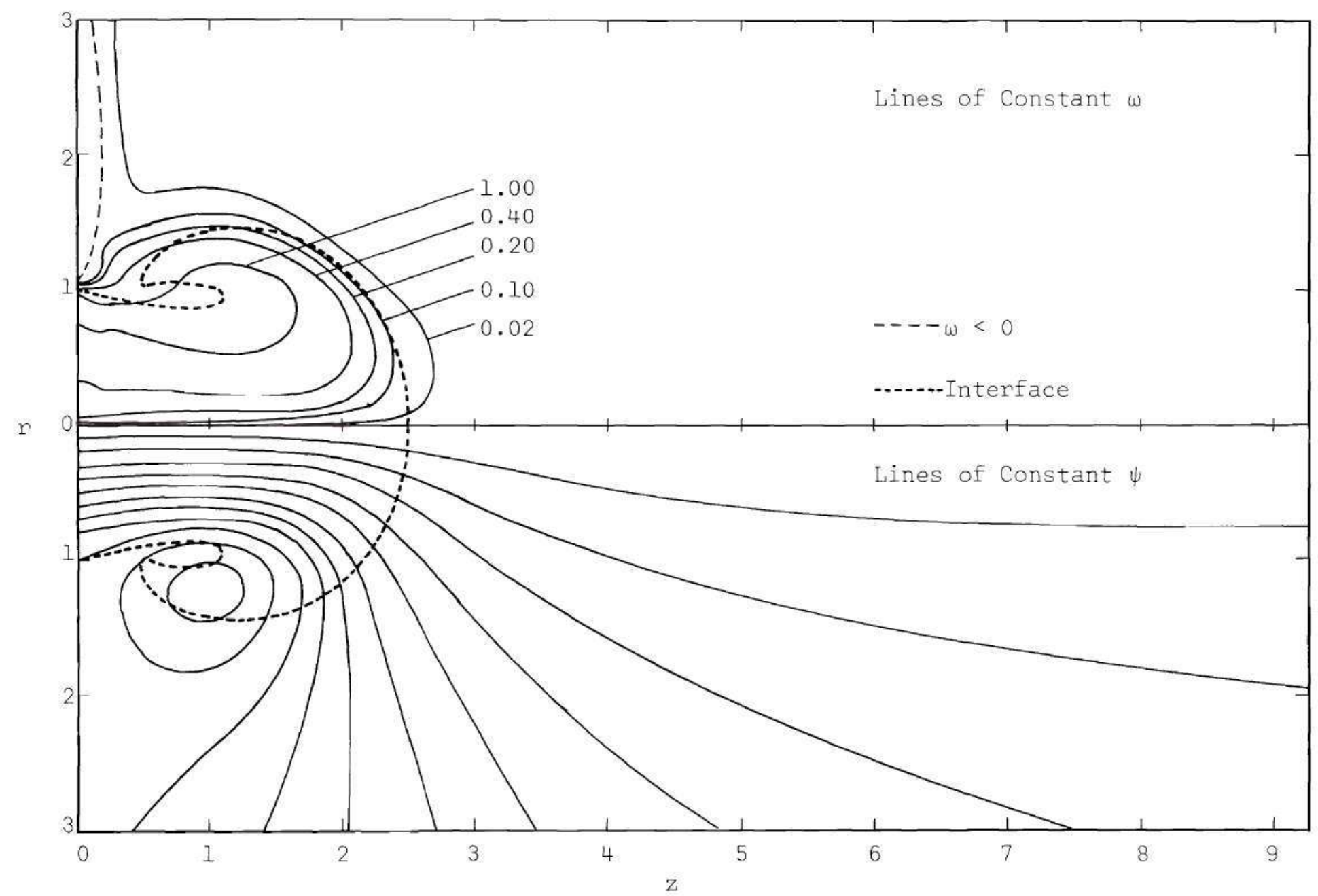

Figure 18. Vorticity Distribution and Streamline Pattern at $t=12$-- Parabolic Profile 


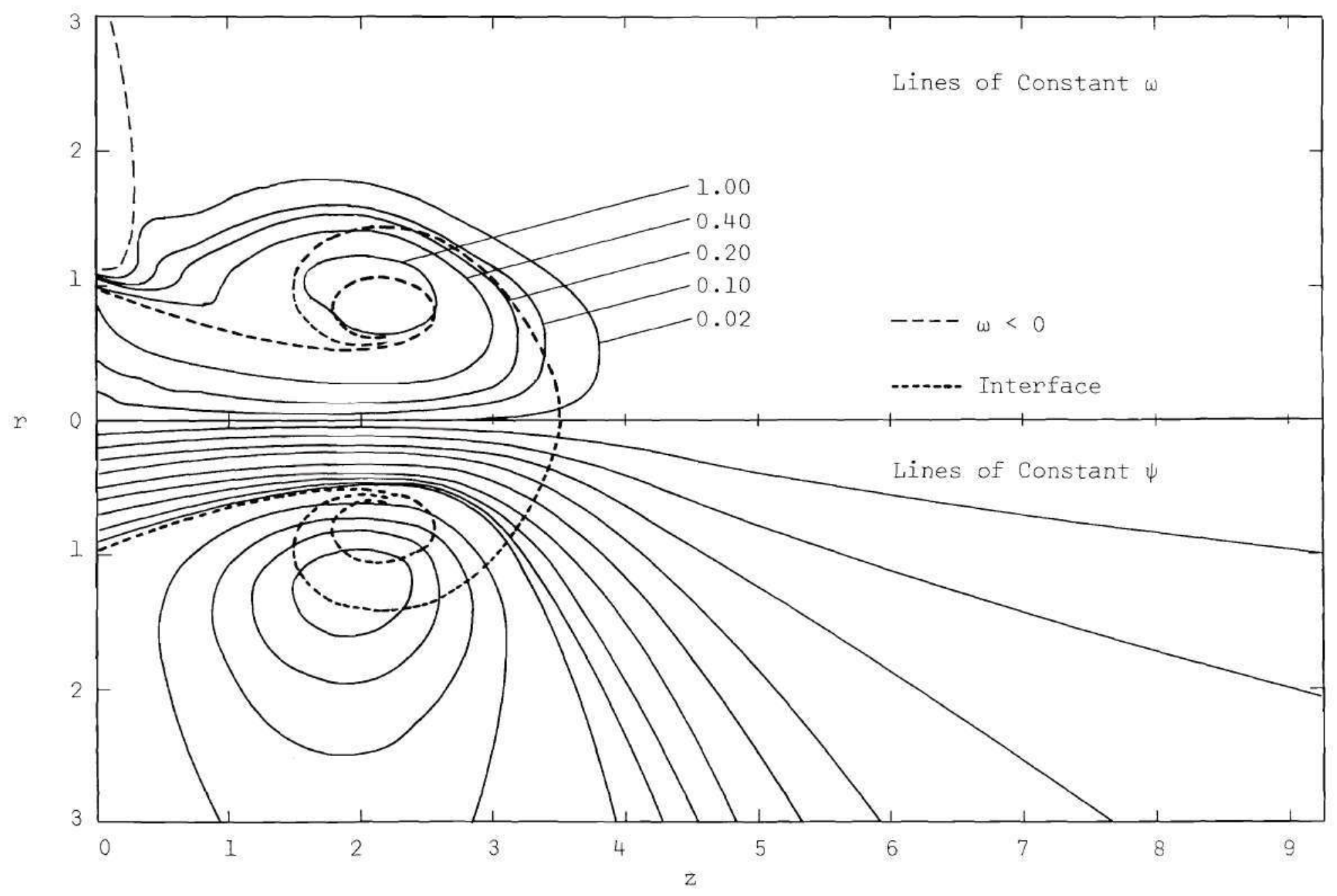

Figure 19. Vorticity Distribution and Streamline Pattern at $t=16$-- Parabolic Profile 


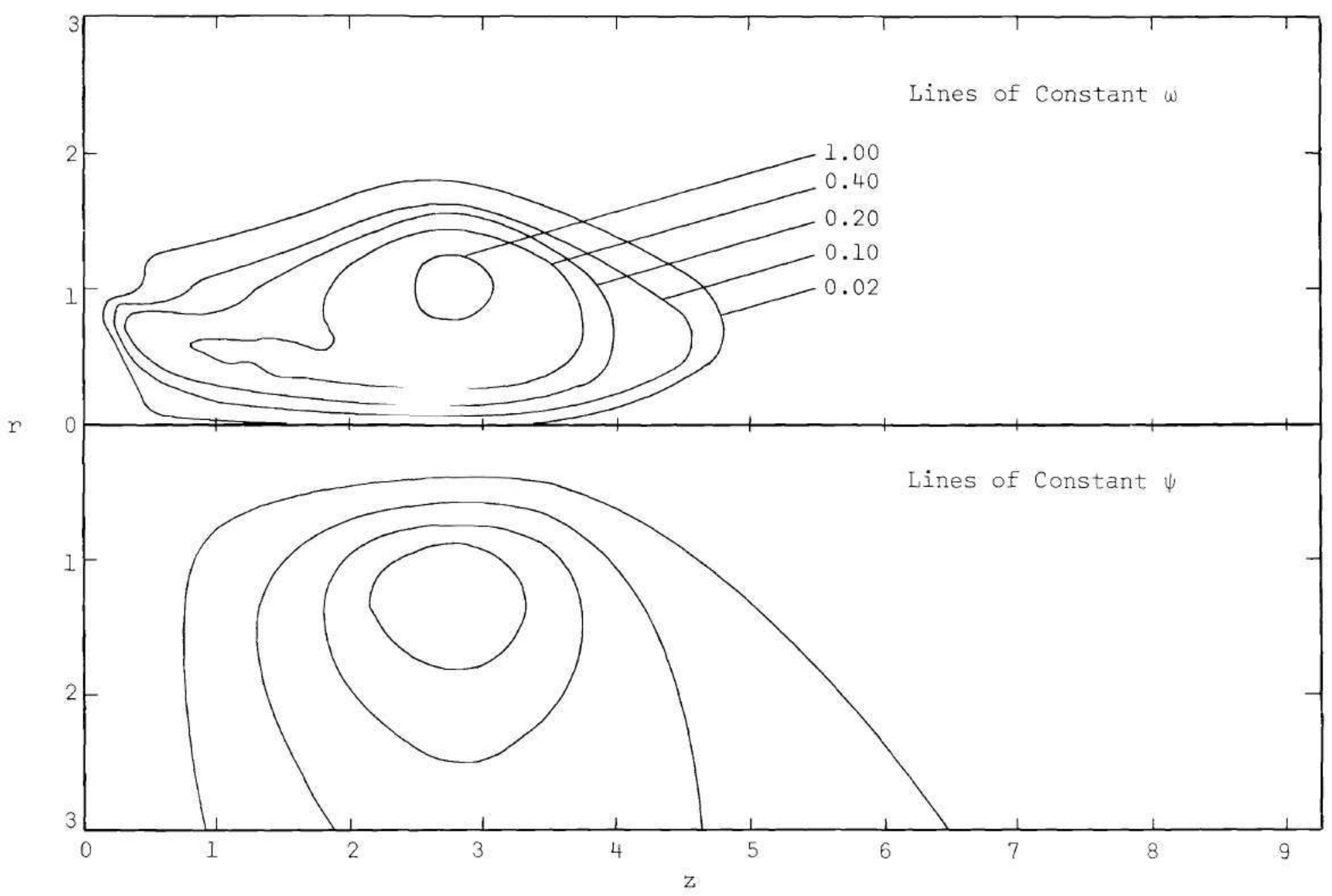

Figure 20. Vorticity Distribution and Streamline Pattern at $t=20$-- Parabolic Profile 


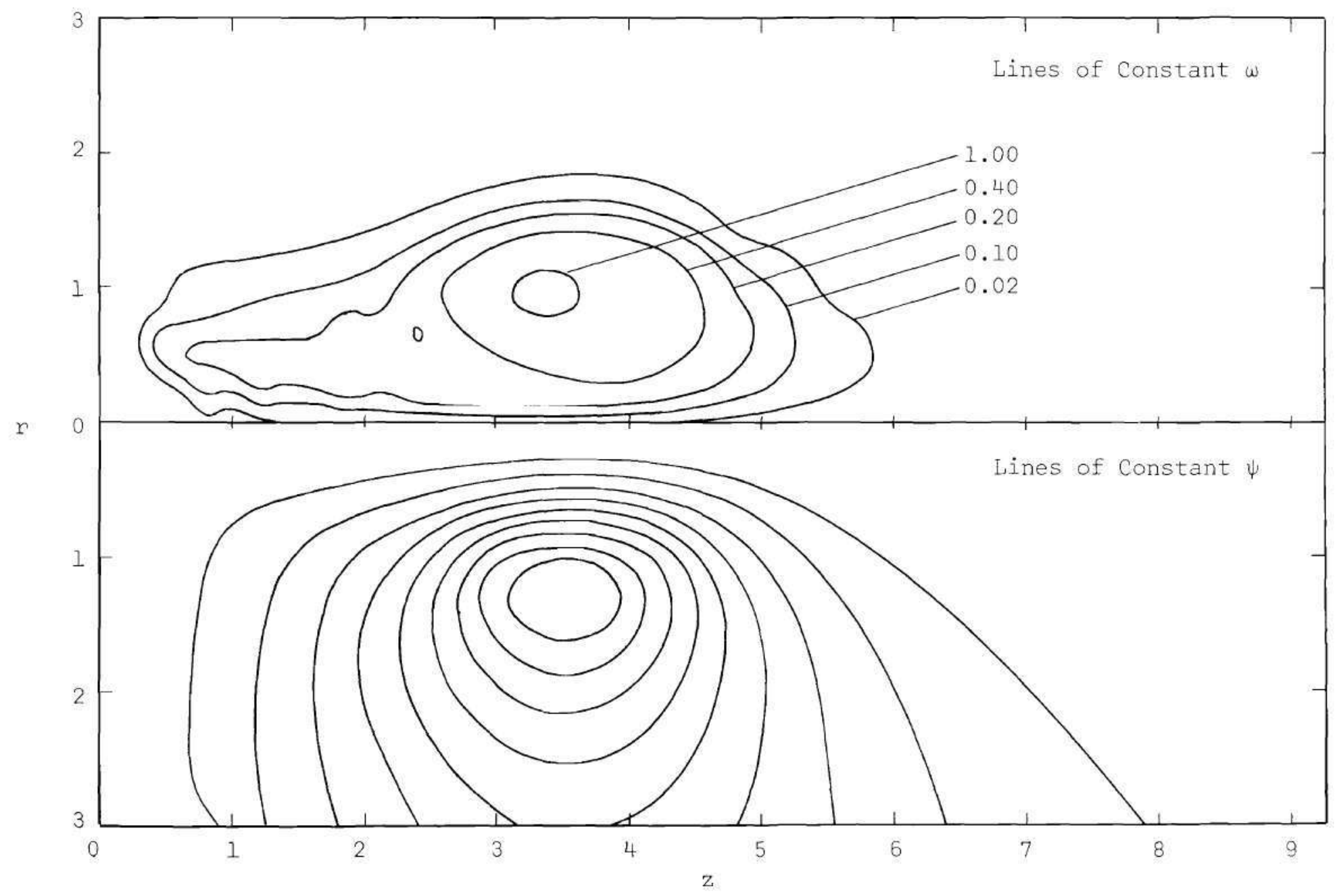

Figure 21. Vorticity Distribution and Streamline Pattern at $t=24$-- Parabolic Profile 


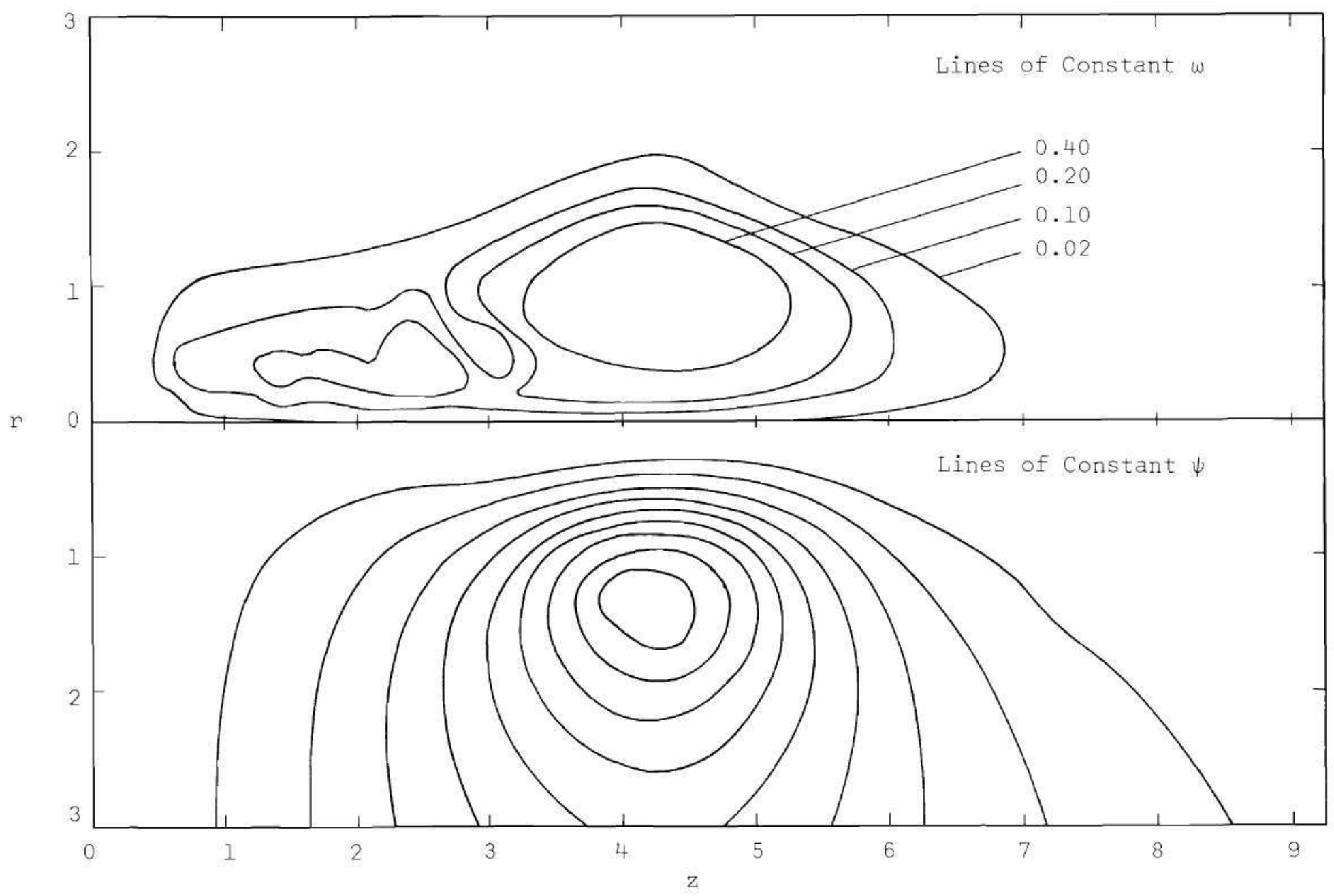

Figure 22. Vorticity Distribution and Streamline Pattern at $t=28$-- Parabolic Profile 


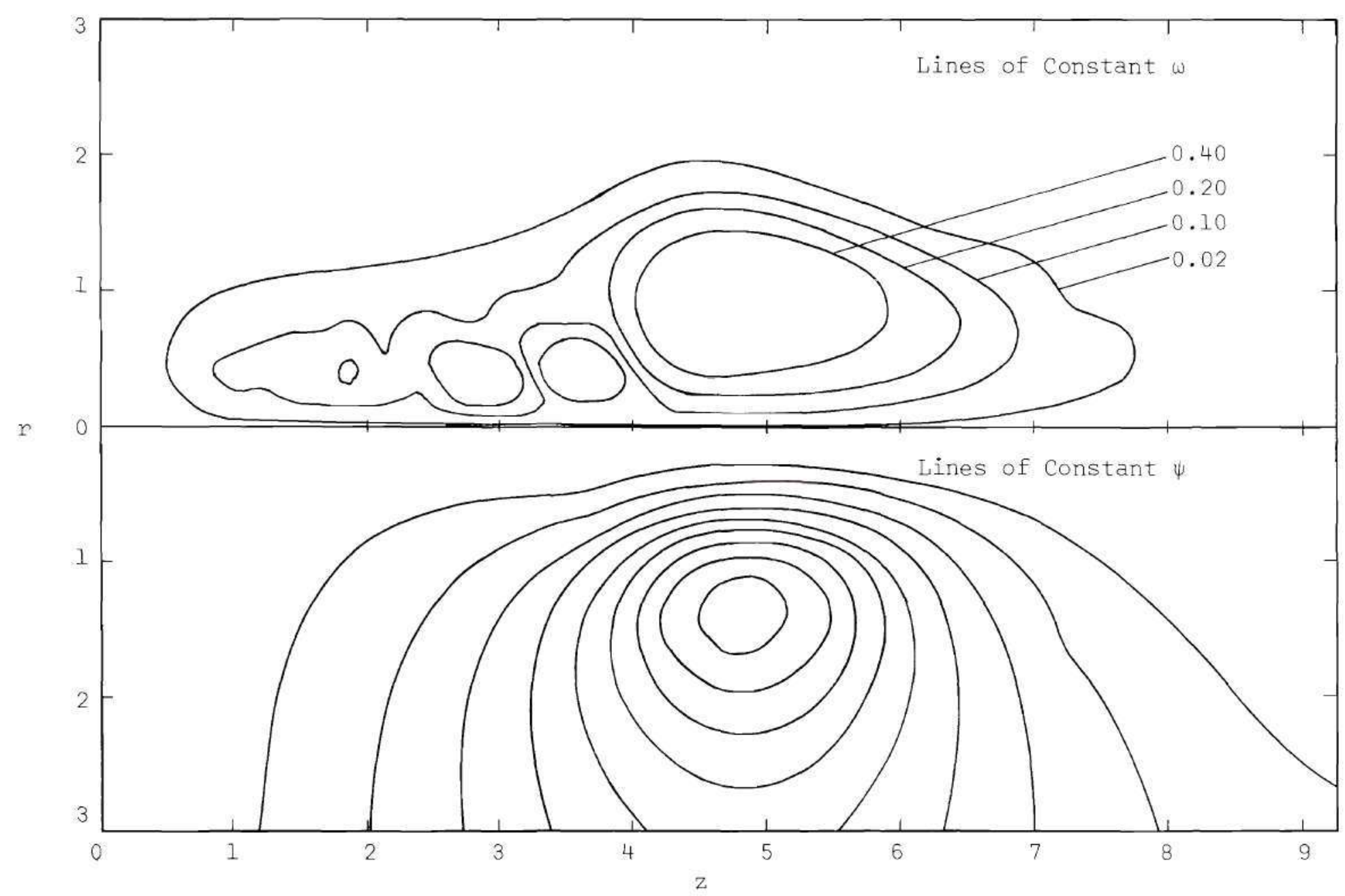

Figure 23. Vorticity Distribution and Streamline Pattern at $t=32$-- Parabolic Profile 


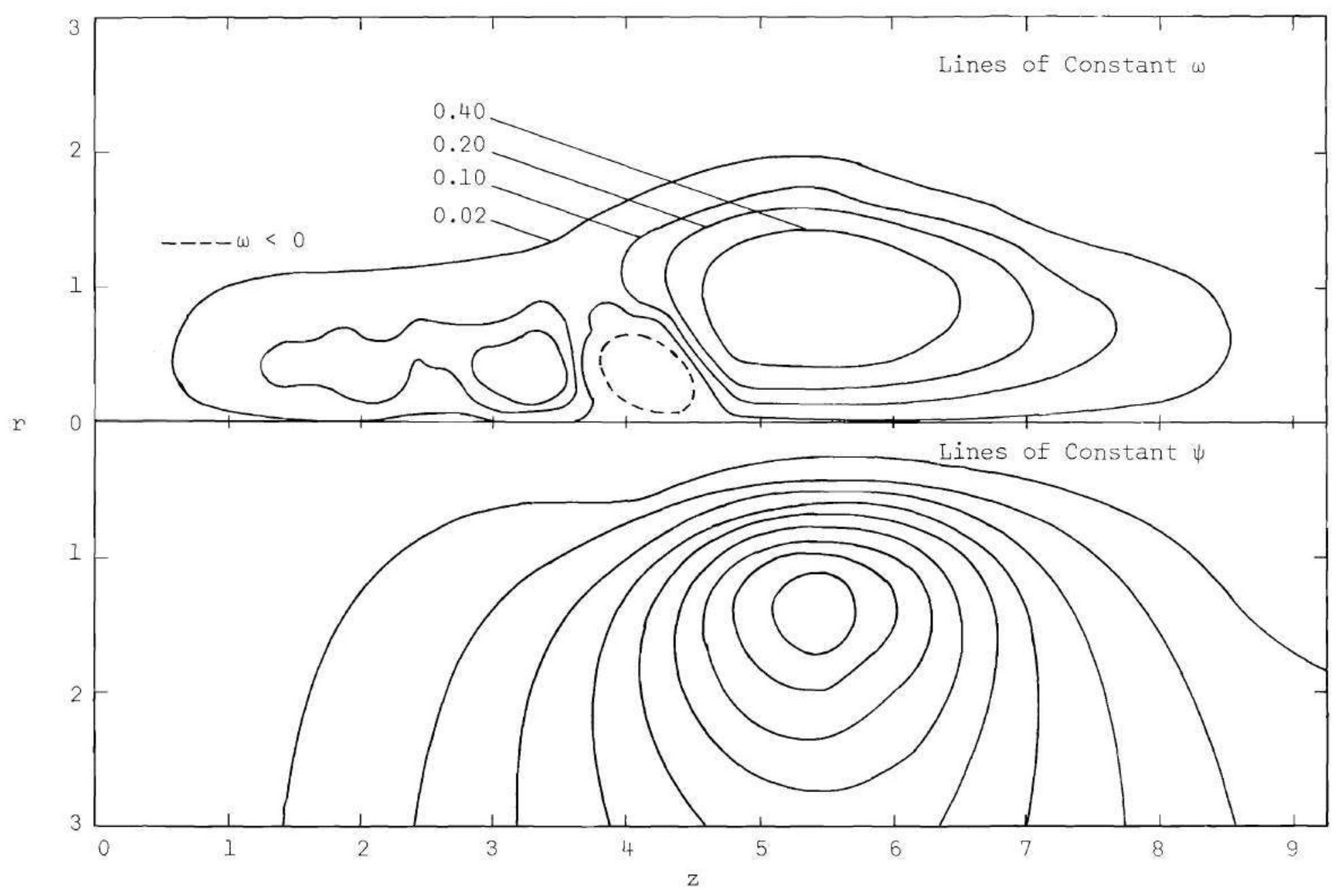

Figure 24. Vorticity Distribution and Streamline Pattern at $t=36$-- Parabolic Profile 


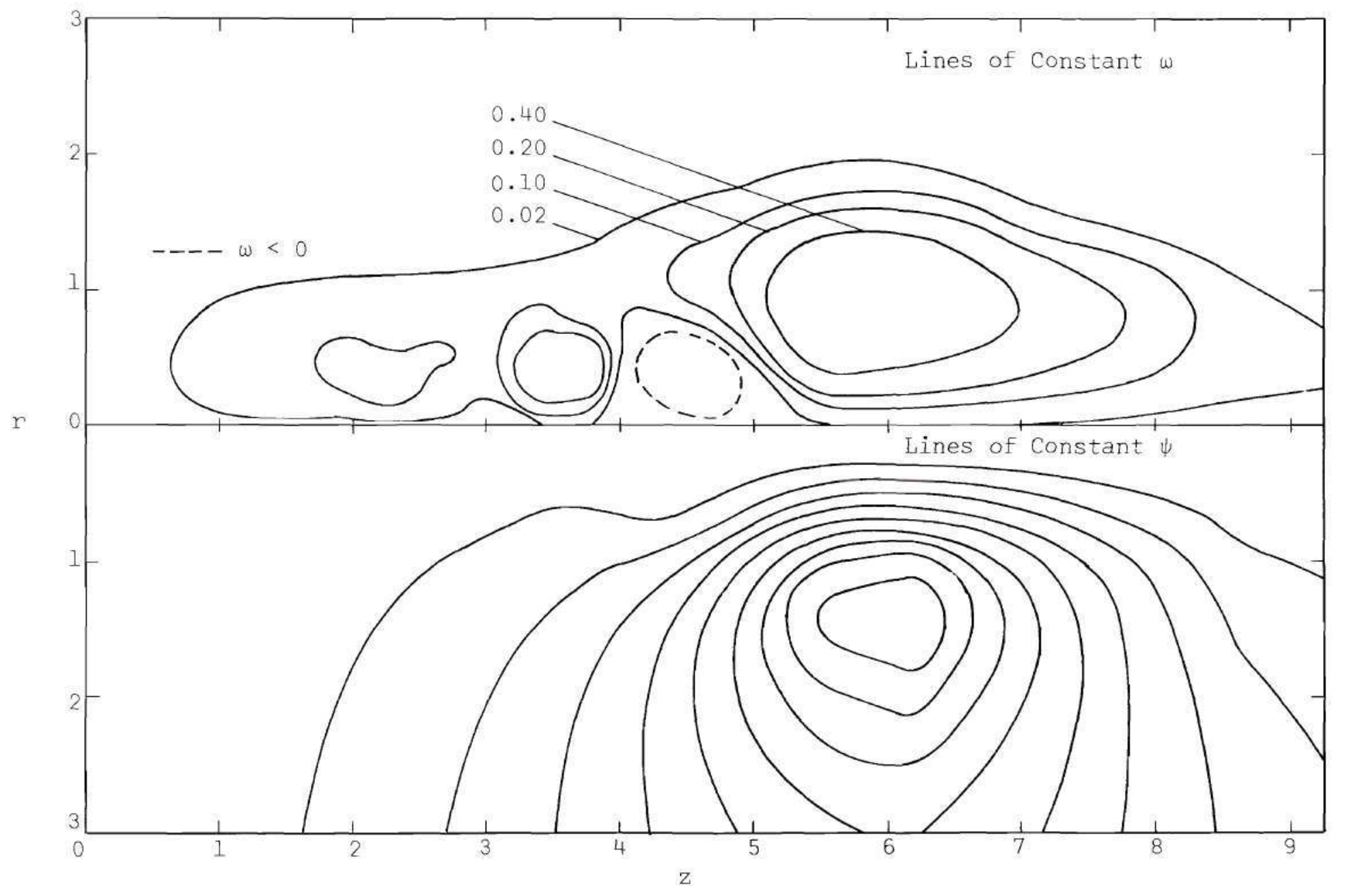

Figure 25. Vorticity Distribution and Streamline Pattern at $t=40--$ Parabolic Profile 


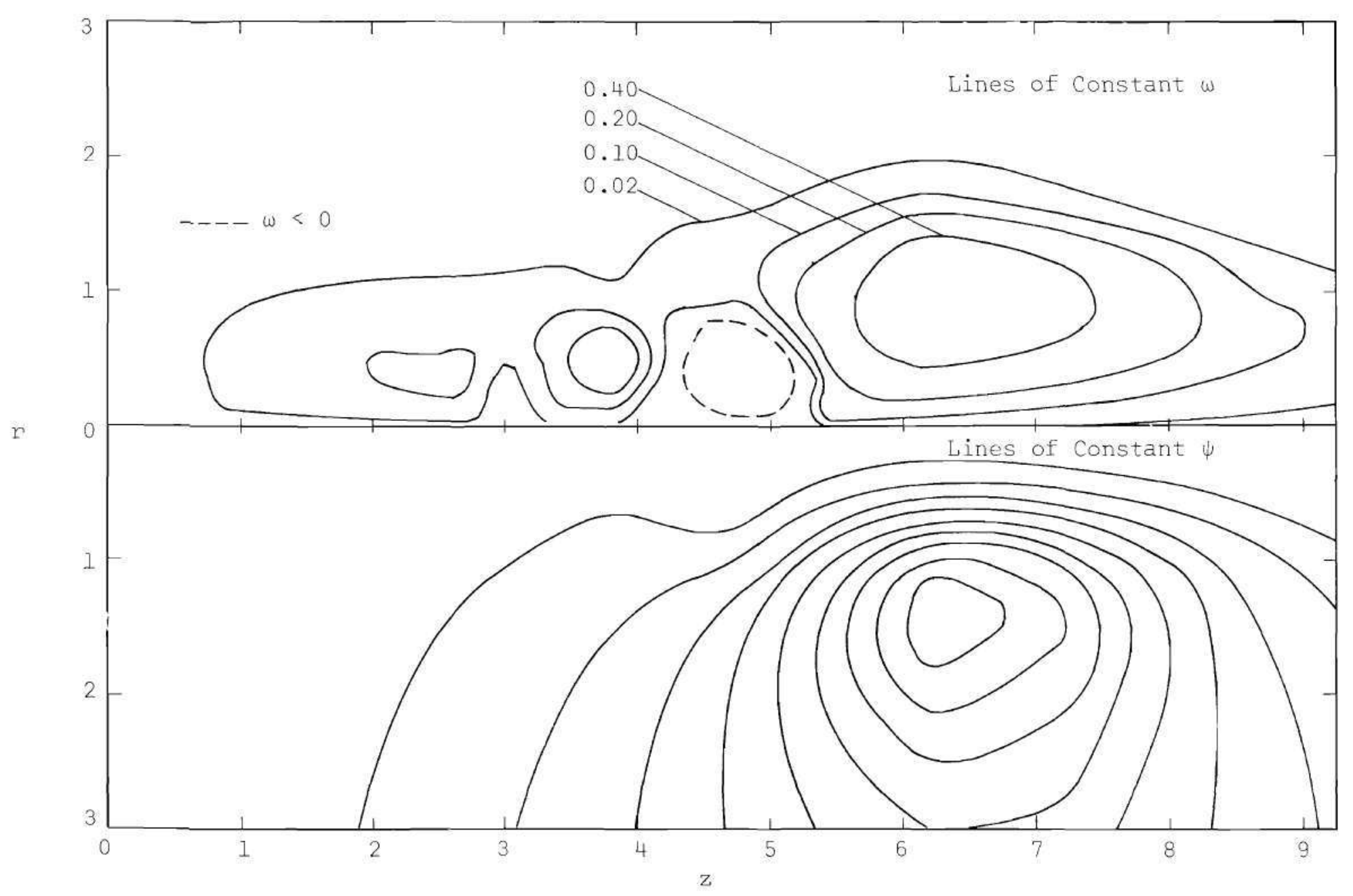

Figure 26. Vorticity Distribution and Streamline Pattern at $t=44$-- Parabolic Profile 


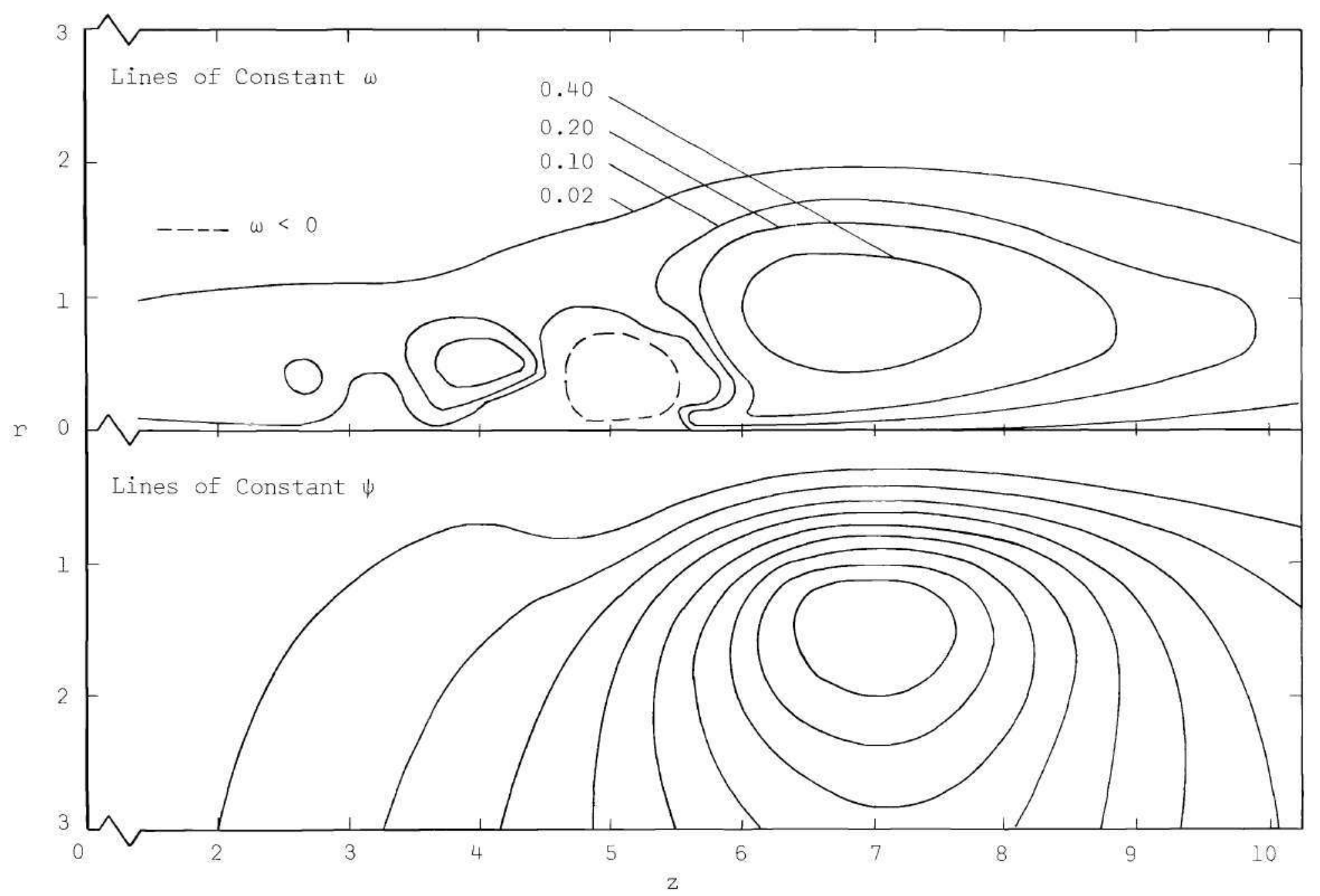

Figure 27. Vorticity Distribution and Streamline Pattern at $t=48$-- Parabolic Profile 


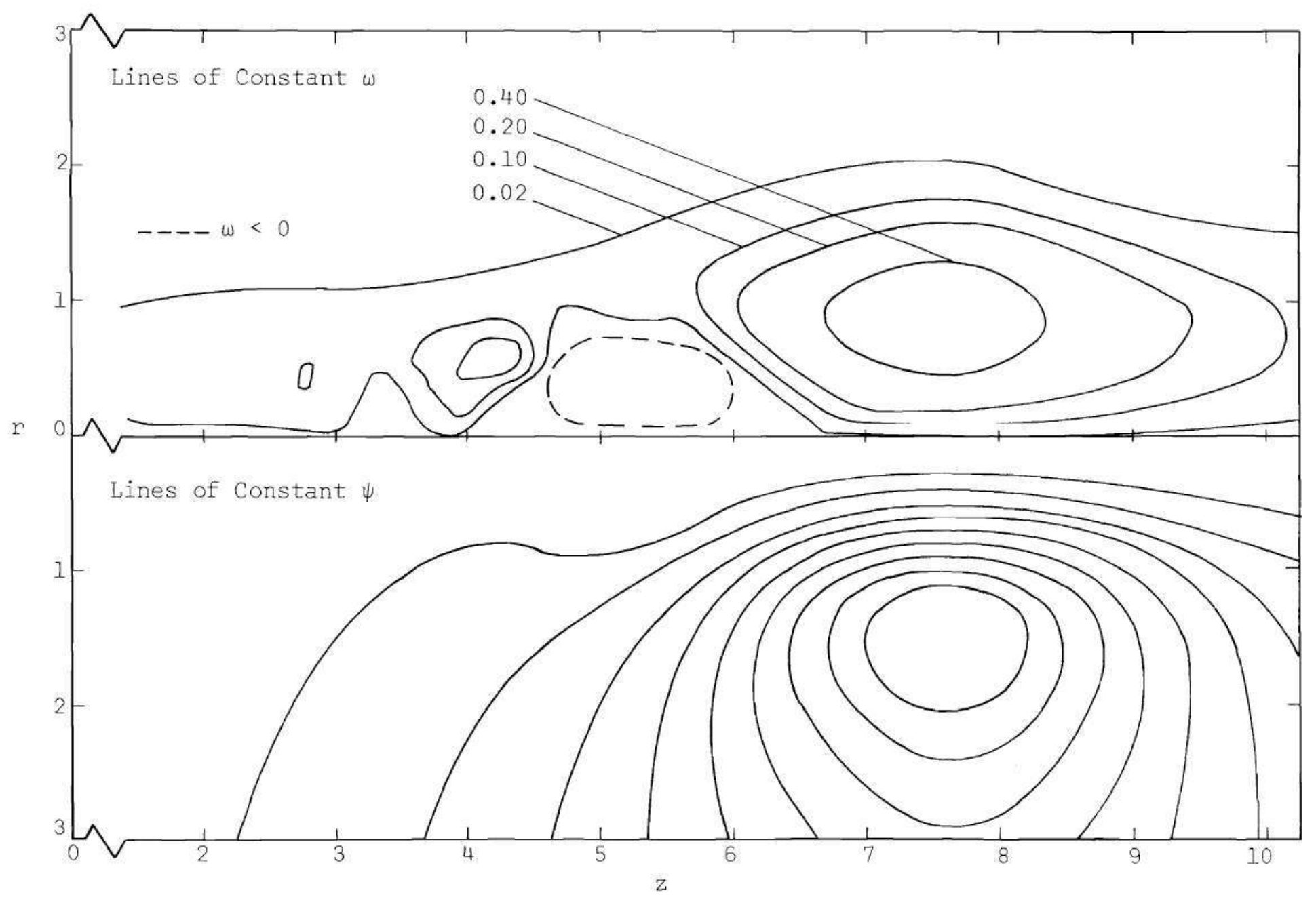

Figure 28. Vorticity Distribution and Streamline Pattern at $t=52$-- Parabolic Profile 


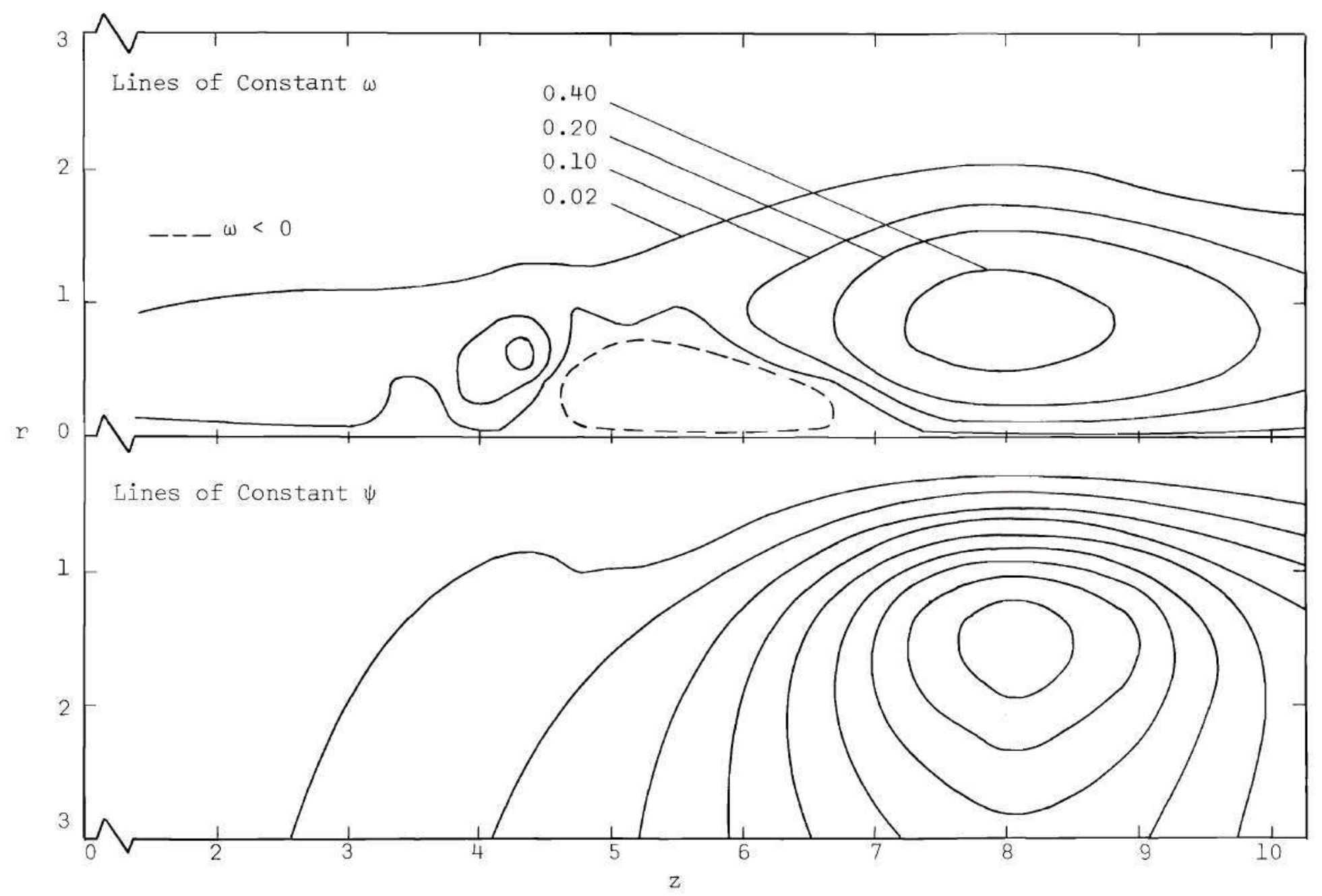

Figure 29. Vorticity Distribution and Streamline Pattern at $t=56$-- Parabolic Profile 
Table 3. Values of the Stream Function for the Plotted Streamlines--Parabolic Profile Case

\begin{tabular}{|c|c|c|c|c|c|c|c|}
\hline Index & $t=4.0$ & $t=8.0$ & $t=12.0$ & $t=16.0$ & $t=20.0$ & $t=24.0$ & $t=28.0$ \\
\hline 1 & $1.99(-3)^{*}$ & $3.98(-3)$ & $3.98(-3)$ & $1.99(-3)$ & $5 \cdot 23(-2)$ & $2.49(-2)$ & $2.49(-2)$ \\
\hline 2 & $7.84(-3)$ & $1.57(-2)$ & $1.57(-2)$ & $7.84(-3)$ & $1.05(-1)$ & 4. $98(-2)$ & $4.97(-2)$ \\
\hline 3 & $1.72(-2)$ & $3.44(-2)$ & $3.44(-2)$ & I. $72(-2)$ & $1.57(-1)$ & $7 \cdot 47(-2)$ & $7.46(-2)$ \\
\hline 4 & $2.94(-2)$ & $5.89(-2)$ & $5.89(-2)$ & $2.94(-2)$ & $2.09(-1)$ & $9.97(-2)$ & $9.94(-2)$ \\
\hline 5 & 4. $38(-2)$ & $8.75(-2)$ & $8.75(-2)$ & 4. $38(-2)$ & & $1.25(-1)$ & $1.24(-2)$ \\
\hline 6 & $5.90(-2)$ & $1.18(-1)$ & $1.18(-1)$ & $5.90(-2)$ & & 1. $49(-1)$ & 1. $49(-2)$ \\
\hline 7 & $7 \cdot 40(-2)$ & $1.48(-1)$ & $1.48(-1)$ & $7 \cdot 40(-2)$ & & $1.74(-1)$ & $1.74(-2)$ \\
\hline 8 & $8.70(-2)$ & $1.93(-1)$ & $1.93(-1)$ & $8.70(-2)$ & & $1.99(-1)$ & $1.99(-1)$ \\
\hline 9 & & $2.00(-1)$ & $2.00(-1)$ & $9.64(-2)$ & & $2.24(-1)$ & $2.24(-1)$ \\
\hline 10 & & $2.05(-1)$ & $2.31(-1)$ & $1.35(-1)$ & & & \\
\hline 11 & & & $2.62(-1)$ & $1.70(-1)$ & & & \\
\hline 12 & & & & $2.06(-1)$ & & & \\
\hline 13 & & & & $2.41(-1)$ & & & \\
\hline
\end{tabular}

\begin{tabular}{cccccccc} 
Index & $t=32.0$ & $t=36.0$ & $t=40.0$ & $t=44.0$ & $t=48.0$ & $t=52.0 \quad t=56.0$ \\
\hline & & & & & & \\
1 & $2.42(-2)$ & $2.34(-2)$ & $2.19(-2)$ & $2.15(-2)$ & $1.95(-2)$ & $1.93(-2)$ & $1.95(-2)$ \\
2 & $4.83(-2)$ & $4.68(-2)$ & $4.38(-2)$ & $4.29(-2)$ & $3.90(-2)$ & $3.86(-2)$ & $3.89(-2)$ \\
3 & $7.25(-2)$ & $7.02(-2)$ & $6.57(-2)$ & $6.44(-2)$ & $5.85(-2)$ & $5.79(-2)$ & $5.84(-2)$ \\
4 & $9.67(-2)$ & $9.36(-2)$ & $8.76(-2)$ & $8.59(-2)$ & $7.81(-2)$ & $7.72(-2)$ & $7.78(-2)$ \\
5 & $1.21(-1)$ & $1.17(-1)$ & $1.09(-2)$ & $1.07(-1)$ & $9.76(-2)$ & $9.65(-2)$ & $9.73(-2)$ \\
6 & $1.45(-1)$ & $1.40(-1)$ & $1.31(-1)$ & $1.29(-1)$ & $1.17(-1)$ & $1.16(-1)$ & $1.17(-1)$ \\
7 & $1.69(-1)$ & $1.64(-1)$ & $1.53(-1)$ & $1.50(-1)$ & $1.37(-1)$ & $1.35(-1)$ & $1.36(-1)$ \\
8 & $1.93(-1)$ & $1.87(-1)$ & $1.75(-1)$ & $1.72(-1)$ & $1.56(-1)$ & $1.54(-1)$ & $1.56(-1)$ \\
9 & $2.18(-1)$ & $2.11(-1)$ & $1.97(-1)$ & $1.93(-1)$ & $1.76(-1)$ & $1.74(-1)$ & $1.75(-1)$
\end{tabular}

$* 1.99(-3)=1.99 \times 10^{-3}$ 
vorticity distribution and streamline pattern are shown respectively above and below this line. The radial station $r$ for a particular curve is then the distance between the point in the field and the center line. The vorticity contours shown for the parabolic profile are for values of the dimensionless vorticity equal to $0.02,0.1,0.2,0.4$ and 1.0 . The values of the dimensionless stream functions which correspond to the streamlines plotted are given in Table 3 on the preceding page. At any time, the streamline index of Table 3 may be found for a particular streamline by counting the streamlines on the appropriate figure. The center line is the zero-th streamline.

The lines of constant vorticity (the vorticity contours or isovorts) are lines along which the fluid elements experience the same rotational velocity. The VTE predicts these vorticity distributions and describes how the vortic ity is transported throughout the flow field and how it is diminished by the action of viscosity. In accordance with the Helmholtz vortex theorems, ali vorticity must be produced at the boundaries of the flow field. Since in this problem the vorticity is zero on all boundaries but one, the velosity gradients in the exit plane and on the solid boundary generate all of the vorticity in the flow field. Most of the vorticity contours plotted correspond to positive values of vorticity which indicate counter-clockwise rotation in the upper half of each figure. This is consistent with the rotation already observed in the streakline patterns of earlief figures. However, on several of the figures, the existence of certain regions of negative vorticity are indicated by dashed contours. In addition, on the figures corresponding to 
the early stages of vortex development, the interface between the smoke from the exit plane and the ambient surroundings is also indicated.

The streamline patterns have already been mentioned earlier with respect to Figure 15. For the following discussion, however, consider Figure 18 which describes the flow at $t=12.0$. These contours correspond to positive values of the stream function, and they indicate clockwise rotation in the lower half of the azimuthal plane. In some of the later plots, a region appears near the center line over which the stream function is negative. This indicates counter-clockwise rotation in the lower half of the plane, and it corresponds to the region of reversed angular velocity mentioned above in the discussion of negative vorticity

For this study, a stream channel is defined as the region between adjacent streamlines. Figure 18 shows streamlines emanating from the. exit plane at increments of $1 / 10$ of the radius of the exit plane. Since the velocity profile in the exit plane is parabolic, adjacent stream channels which emerge from the exit plane do not indicate equal rates of mass flow.

In contrast to these contours is the group of closed streamlines which envelop regions of the flow and thus indicate the presence of the vortex ring. Here the streamlines are given by equal increments of the stream function, and adjacent stream channels do indicate equal mass flow rates at any given time. However, the calculation of these equally incremented streamlines is dependent upon the value of the maximum stream function in the field at a particular time. This value decreases with 
time due to viscous dissipation, and the decrease can be seen in Table 3 for $t \geq 20$. Hence, the mass rate of flow between adjacent closed streamlines diminishes with time, and the mass flow rate of stream channels should not be compared quantitatively at different times. The phenomenon of the generation and development of a viscous vortex ring is illustrated in Figures 16 through 29. Figure 16 shows the flow field at $t=4.0$ at which time the velocity in the exit plane has existed for only a short period. The region of highest vorticity occurs near the edge of the exit plane. The streamline pattern indicates that the environment around the smoke is moving slowly away from the exit plane.

In Figure 17 the velocity in the exit plane is rapidly approaching a maximum. The region of greatest vorticity has spread in both the axial and radial directions as the effect of the surroundings on the fluid emerging from the exit plane is felt in more and more of the smoke. The vortex ring is already forming, and this is indicated by the closed streamine just outside the exit plane. Fluid is moving along the wall toward the jet. This motion corresponds to a negative axial derivative of the radial velocity, a term which is the only component of vorticity on the soild boundary. This region of negative vorticity is indicated by the dashed contour near the solid boundary. The flow field at the time $t=12$ is illustrated in Figure 18 . The ring is traveling quite rapidly out into the field now. The region of highest vorticity has spread radially outward to encompass the center of the vortex ring. The vorticity contour for $\omega=1,0$ is beginning to pinch off near the exit plane. This is the result of two causes. 
First, the velocity in the exit plane is decreasing, and this in turn causes a decrease in the vorticity in the adjacent region. Second, the large amount of air moving radially inward near the wall corresponds to the large region of negative vorticity which separates the positive vorticity of the vortex ring from the solid boundary. It is the opinion of the author that this separation of the region of positive vorticity from the wall marks the time after which the center of the vortex may be ascertained by the observation of the vorticity distribution. The vortex center corresponds to the point showing the highest vorticity in the field.

Assume that at a particular time both the location of the vortex center and the vorticity at the vortex center are known. The vorticity at this point is the rotational velocity of an infinitesimally small region which rotates as a solid body. (This idea is similar to the concept of a Helmholtz vortex, except that here the radius of the solid core is not only small but also infinitesimal.) Somewhere at a large distance from the vortex, the surrounding fluid is essentially at rest. Momentum is transferred by the shear stresses of the viscous fluid from the high vorticity flow at the vortex center to the surroundings. Therefore, as an observer moves away from the vortex center into the surroundings, the velocity gradients (hence, the vorticity) decrease. If the vorticity diminishes in all directions away from the ring center, then the vorticity at the center must be a maximum. At the time $t=12$ which is illustrated by Figure 18, the region of highest vorticity is separating from the boundary. The point of maximum vorticity must lie somewhere within the contour for $\omega=1$, and this point corresponds to 
the vortex center.

Consider now the streamline pattern of Figure 18. This corroborates the fact that the ring is forming and the velocity in the exit plane is decreasing. The latter observation is verified by the converging of the streamlines as they leave the exit plane. The velocity increases near the axial location of the vortex center in the region occupied by the fluid elements which emanated from the exit plane at the time of its maximum velocity. Downstream of these fluid particles the velocities again decrease, and near the vortex the elements start the long journey around it and away from the center line.

Next, consider Figure 19 at the time $t=16$. Here the region of the highest vorticity plotted, $\omega=1.0$, has separated from the boundary, and the contour of the next highest vorticity, $\omega=0.4$, is being pinched off from the boundary. The region on and near the solid boundary exhibits a negative vorticity which reflects the flow of air along the solid boundary toward the center line. The velocity in the exit plane is still decreasing, and correspondingly the streamlines originating in the exit plane converge more than they did at earlier times. The highest velocities in the field occur near the center line at the axial location of the center of the vortex ring.

Figure 20 illustrates the flow at the instant when the impulsive velocity vanishes. The vorticity contours plotted are separated from the boundary. Viscosity is diminishing the higher velocity gradients in the field. The contour for $\omega=1.0$ is growing smaller, and the contour for $\omega=0.02$ is growing larger as vorticity is being diffused throughout the field. The only streamlines remaining are the closed ones which 
indicate the presence of the ring. The streamline pattern is not symmetrical, and it is flattened near the boundary indicating that the solid boundary still has a considerable effect on the vortex. This is also shown in the vorticity distribution which gives fuller vorticity contours near the wall.

At time $t=24$, Figure 21 continues to exhibit the phenomenon of vorticity diffusion as the shear stresses decrease the velocity gradients in the flow. It is interesting to note that the trailing portions of the vorticity distribution tend to remain adjacent to the wall where the velocities are now quite small. In the meantime, the vortex ring has traveled downstream. A few more streamlines have been added to the field to present a more detalled description of the flow. The boundary at $z=0$ and the center line form one streamline. Recall that ali other streamlines are plotted for equal increments of the stream function, so all the stream channels (the regions between adjacent streamlines) represent equal instantaneous rates of mass flow. The streamlines now show a similarity with those associated with a vortex ring composed of a single circular vortex filament as given by Ringleb [9] for example. An iliustration of these streamiines will be given at the end of this section. However, in contrast to the streamlines about a single vontex filament without a wall constraint, the streamilines at a large distance from the center of the pattern of Figure 21 are not symmetrical about a plane which is normal to the center line and which passes through the axial location of the vortex center.

Figure 22 at the time $t=28$ shows a separation of the vorticity regions. This separation is marked by a slignt irregularity of the 
streamline closest to the center line at $z \stackrel{\sim}{=}$. The vortex ring is still moving downstream, and the streamline pattern continues to remain of the same general shape and size. The region of highest vorticity given by the contour for $\omega=1.0$ has disappeared.

Another separation of the vorticity contours occurs at the time $t=32$ in Figure 23, and the vorticity is diffusing more rapidly. The region which separated in the previous figure is now identified by the small area of positive vorticity enclosed by the contour $\omega=0.02$. The gradients here are decreasing rapidly. The reason for this action may be understood by studying the region of the flow field near the center line at $\mathrm{z} \cong 3.5$. Upstream, a region of relatively high positive vorticity corresponds to rotation in a counter-clockwise direction. The same is true in the region downstream. Meanwhile, the portion of fluid between these two regions is losing its vorticity very quickly. The counter-clockwise velocities on either side should cause a clockwise velocity in between.

Figure 24 shows that this is indeed the case. An embedded region of negative vorticity has appeared near the center line at $z \stackrel{2}{=} 4$. This indicates that a region of the flow above the center line is rotating in a clockwise direction. A secondary vortex has been produced whose sense is opposite to that of the vortex ring. The presence of this vortex is also marked by an increased deformation of the streamline closest to the center line. Between this streamline and the center line there actually occurs a region within which the stream function is negative.

Although attention over the last few plots has been focused on the formation of the secondary vortex, the rest of the flow field has 
been undergoing change too. The vorticity is diffusing throughout the fluid, and since the magnitude of the maximum vorticity in the field has been decreasing, the highest value of the stream function in the field has also been decreasing with time. The streamlines are plotted for values of the stream function which correspond to equal increments of the difference between the maximum stream function in the field and the stream function on the center line. Thus, the mass rates of flow between adjacent streamlines are decreasing with time. This may also be seen in the values of the stream function given in Table 3 . In addition, each stream channel is slowly diverging from figure to figure, and this behavior also indicates that the velocities are decreasing. Viscosity is having its effect; the velocities and velocity gradients are diminishing.

In Figure 25, the phenomena occurring in the flow field continue. The rotational velocities of the vortex ring are decreasing. The mass flow between adjacent streamlines decreases with time, and the rotational velocities of the secondary vortex increase somewhat. Figure 26 shows an irregularity in the streamline closest to the center line at $z \simeq 4.5$ as the influence of the secondary vortex is felt in this region.

In the final three figures of this series, Figures 27 through 29, the velocity of the ring is rapidly approaching a constant value. It is important to note that the abscissa of the final figures has been shifted. The velocities within the secondary vortex have reached their peak, and they are now starting to decay. Simultaneously, the irregularity in the streamline closest to the center line is not as lange as it was in Figure 26. The velocities of the fluid elements continue to 
diminish with time.

In the discussion of the generation and development of the vortex ring just concluded, no attempt was made to indicate the point in time at which the vortex ring had formed. Okabe and Inoue [30] seem to think that the formation of the vortex is complete when the hole appears in the center of the ring cross-section. No such clearly-defined time is evident from the series of figures just presented; the generation and development of a vortex ring is a continuous process. Velocity Profiles

Figure 30 shows the axial velocity distribution on a radial line near the axial location of the vortex center. The figure illustrates the large velocity gradients occurring in the flow. The velocity at the center line is about half of the maximum velocity (i.e., $v_{z}(0,0)=1.0$ at $t=10$ ) ever experienced by the flow field. Observation of a smoke ring indicates that after the ring has been generated, the ring velocity appears to remain fairly constant with little dissipation. The large axial velocity near the ring center at the relatively late time of Figure 30 appears to be consistent with the small velocity dissipation which is observed in a smoke ring.

Figure 31 illustrates the large velocity gradients in the axial direction at the same time, $t=40$. It is worthwhile to compare the velocity distribution of Figure 31 to the vorticity distribution and streamline pattern of Figure 25. Near the exit plane the axial velocity is zero, and it increases with increasing $\mathrm{z}$ as the vorticity and clockwise rotational velocity above the center line increase. The center line velocity levels off at $\mathrm{z} \cong 3$ since the vorticity above it has decreased 


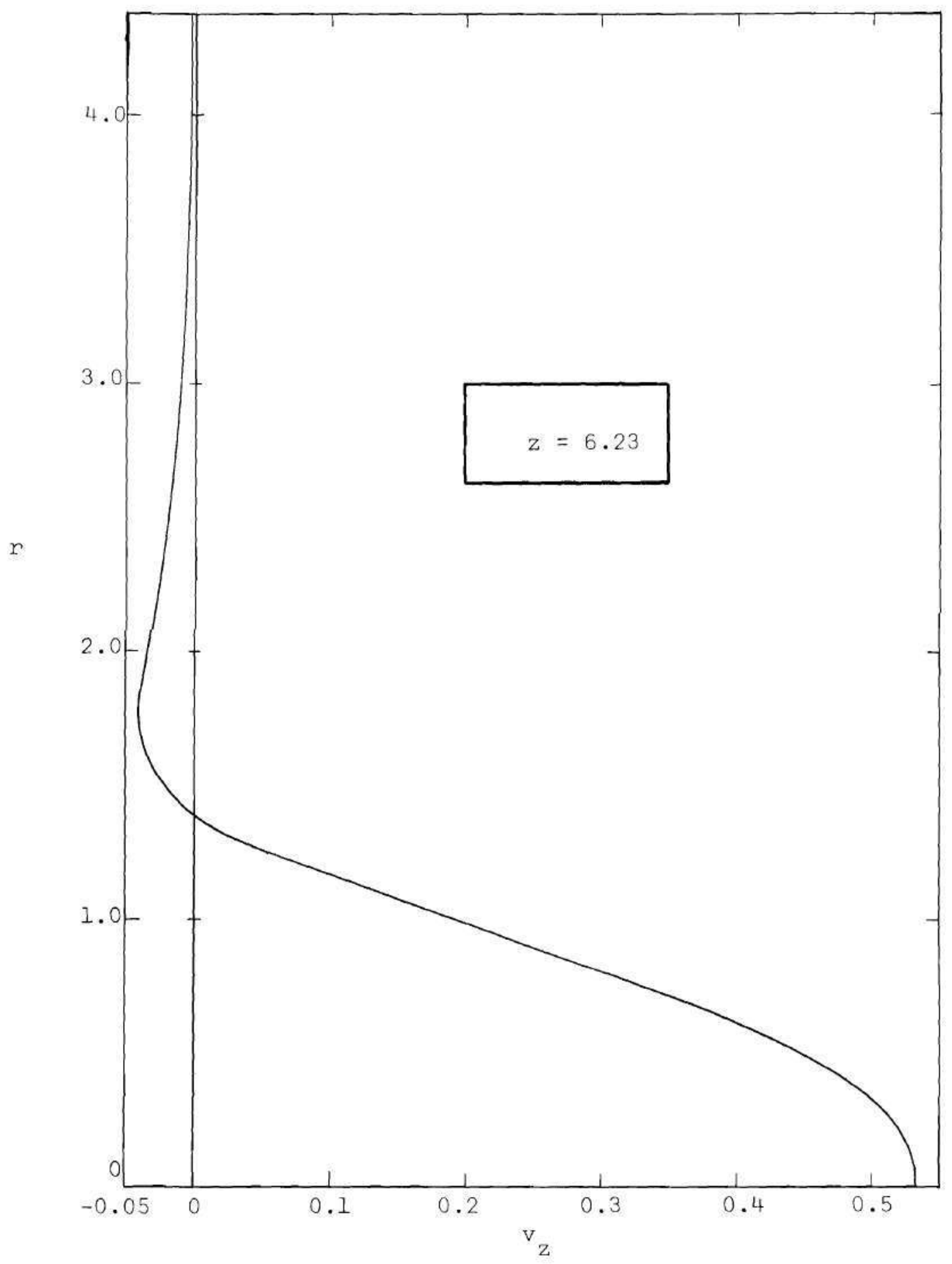

Figure 30. Axial Velocity Distribution near Vortex Center at $t=40-$ Parabolic Profile 


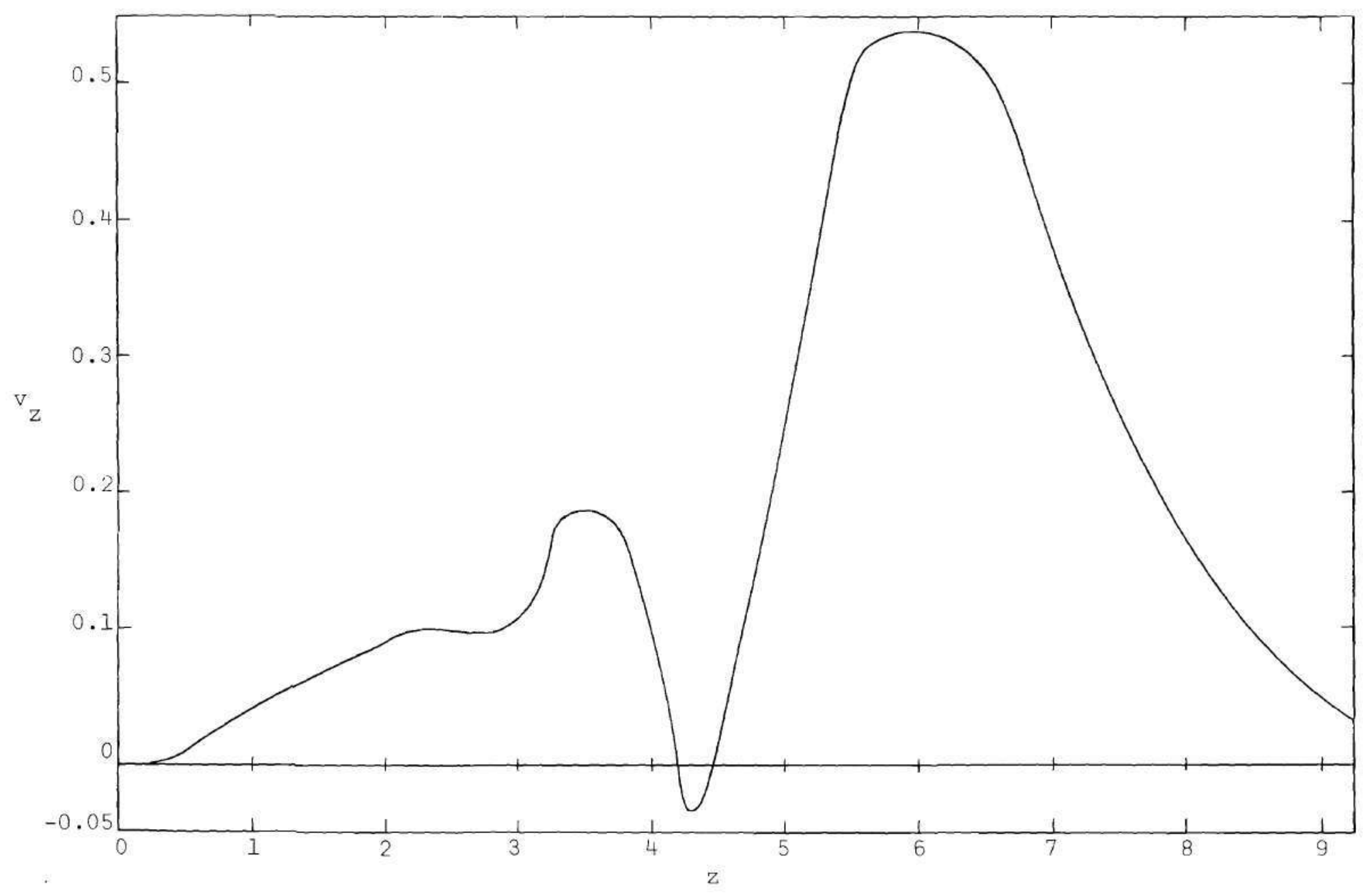

Figure 31. Axial Center Line Velocity at $t=40--$ Parabolic Profile 
in the region between two closed vorticity contours for which $\omega=0.1$. As the higher value of vorticity is approached, the axial velocity again increases to a local maximum which corresponds to the local maximum in vorticity above it. Between $z \cong 4$ and $z \cong 5$, the secondary vortex of reversed flow occurs. This indicates the large decrease in axial velocity (it actually becomes negative near the center of the secondary vortex) and then the large velocity increase as the secondary vortex is passed. The center line velocity reaches its maximum at the center of the vortex ring where the rotational velocity of the field is highest, and then it decays at axial distances further and further removed from the vortex center. This investigation of the center line velocity at a particular time reveals that the velocity field and the vorticity distribution are consistent. Finally, the presence of the negative center line velocity is consistent with the existence of the secondary vortex of reversed flow which was discussed in connection with Figure 25.

Figure 32 illustrates the formation of the secondary vortex and the diffusion of vorticity. The center line velocity is plotted for the times $t$ equal to 36,40 , and 44 . The negative velocity which signifies the reversed flow can be seen developing, and the shifting position of the maximum velocity denotes the movement of the ring in the axial direction. Due to the effect of viscosity, the magnitude of the maximum velocity is decreasing with time, and the radius of curvature of this velocity peak is increasing. This behavior indicates that the velocity gradients and the vorticity are diminishing with time. 


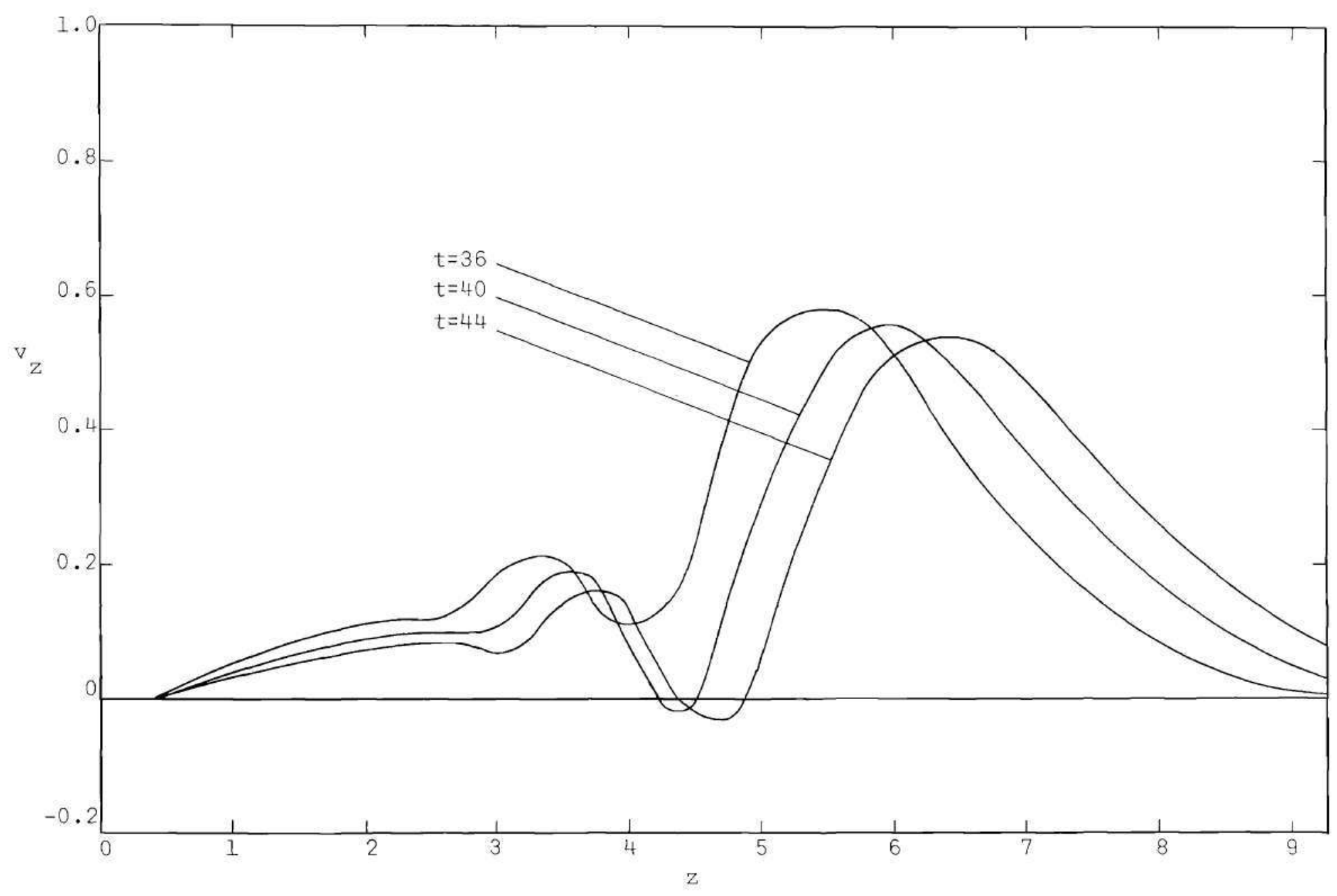

Figure 32. Axial Center Line Velocities at Different Times -- Parabolic Profile 
In Figure 33, the maximum velocity of the flow field is plotted as a function of time. The maximum velocity at any one time occurs on the center line at the axial location of the vortex center as would be expected from the consideration of a single, circular vortex filament. Figure 33 indicates that after a period of time which is approximately three times the impulse time, the maximum velocity in the field has decayed to a value which is a little less than half the peak velocity in the exit plane. The decrease in the velocity begins when the velocity in the exit plane has reached a maximum. Before this time, the maximum velocity occurs in the exit plane as indicated by the linear segment of Figure 33.

Earlier in the discussion, it was mentioned that the closed streamline pattern of the viscous vortex ring was similar to that of a single vortex filament. The latter pattern is illustrated in Figure 34. It can be seen that the streamlines here are comparable to the closed streamlines of Figure 21 through Figure 29.

\section{The Uniform Profile}

The results of the numerical model which employs a uniform profile in the exit plane are presented graphically in the same manner in which they were given for the parabolic profile case. The following figures illustrate streaklines, a representative plot of the velocity vectors and their corresponding streamlines, detailed plots of the vorticity contours and the corresponding streamlines, and finally some typical representations of various velocity distributions. 


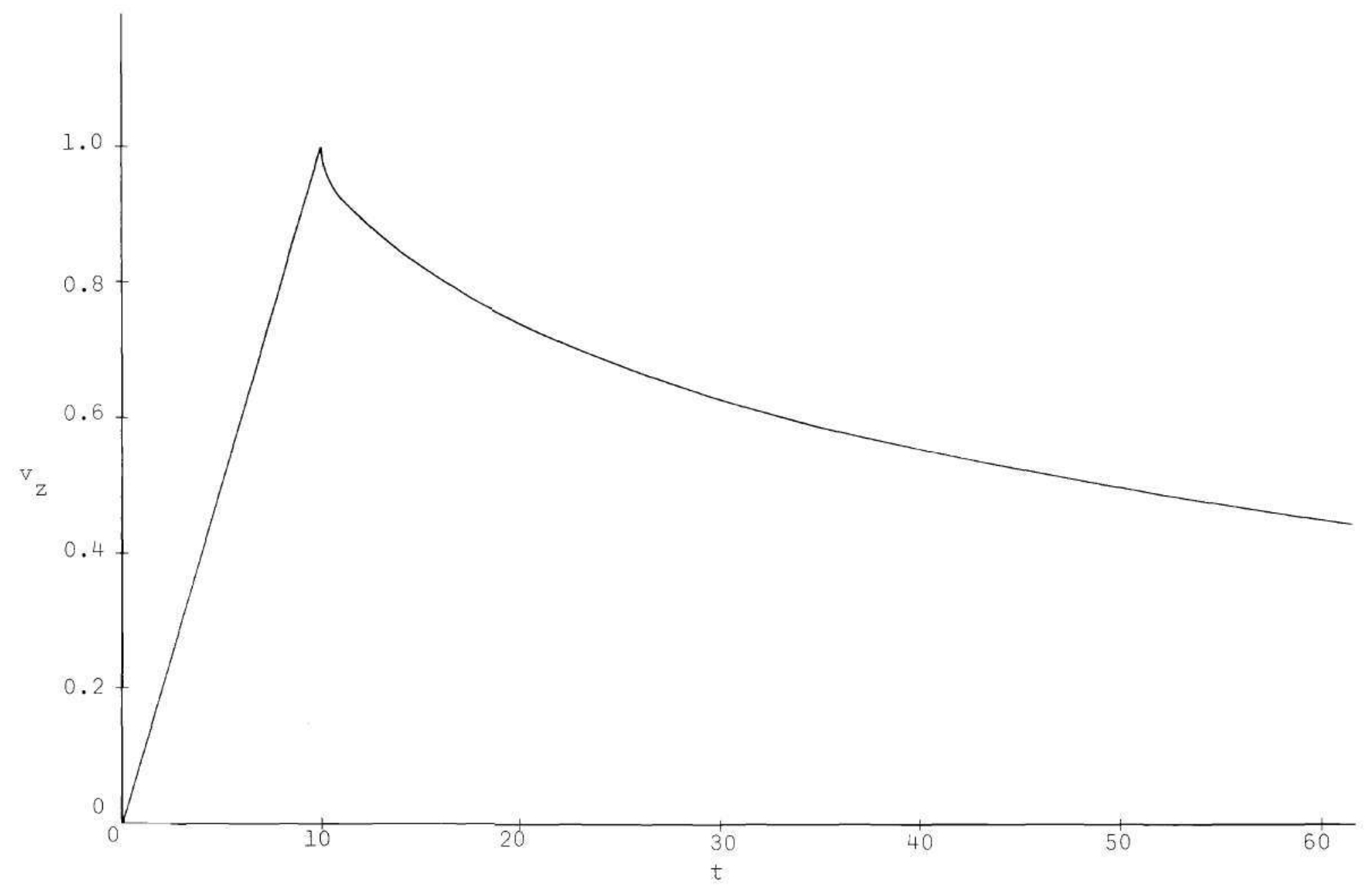

Figure 33. Maximum Center Line Velocity as a Function of Time--Parabolic Profile 


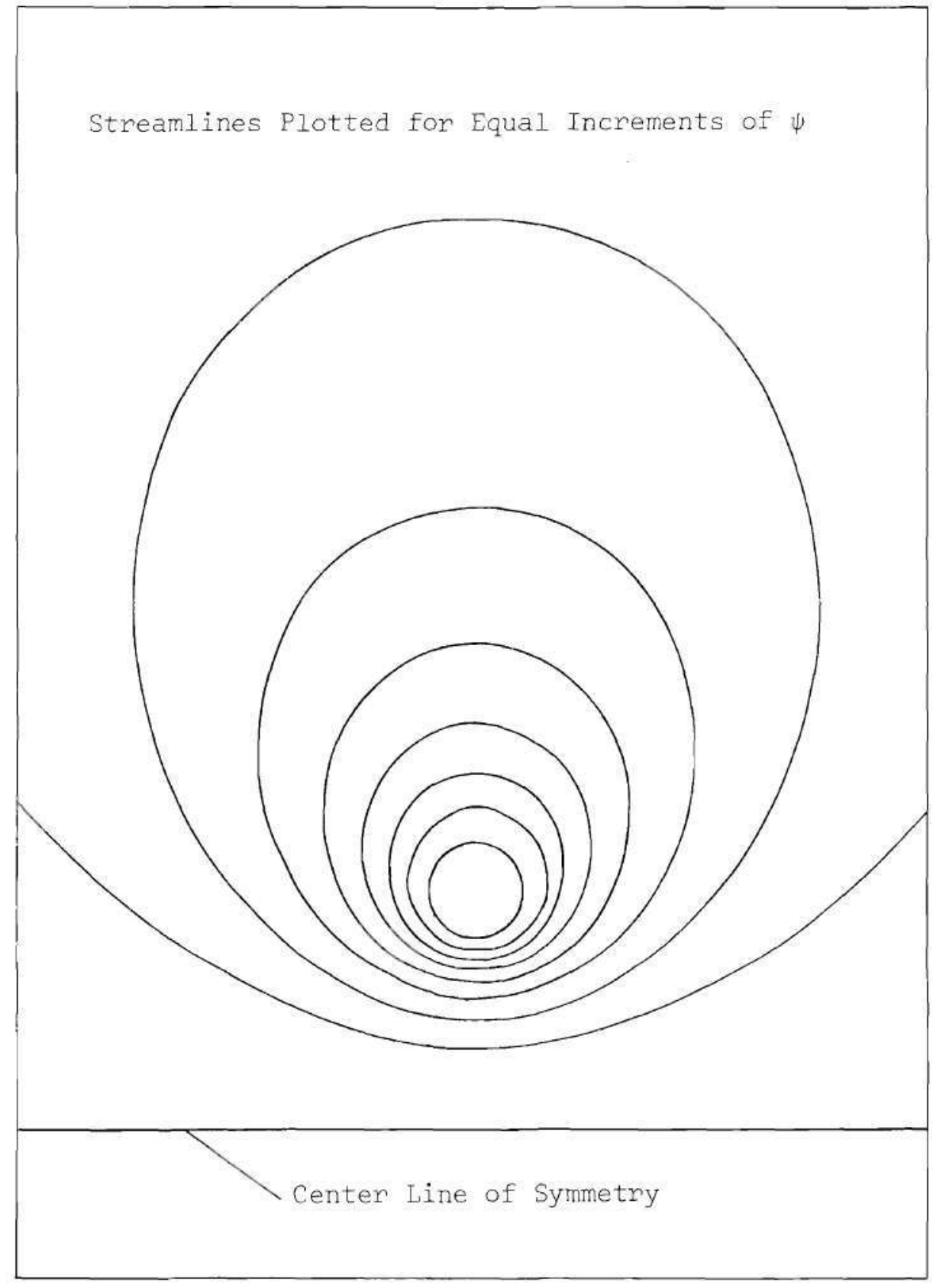

Figure 34. Streamline Pattern about Vortex Filament According to Ringleb [9] 
The Streaklines

As in the case of the parabolic profile, fluid elements are introduced into the flow at various radial stations in the exit plane at each time increment. The distance traversed by a particular element is computed as the product of the velocity at the point occupied by the element and the time increment. In Figures 35 through 38 , the streaklines are given in the $z, r$ plane at the times $t=2,6,10$, and 14 . The dots on the figures illustrate particle locations, and the dashed line denotes the interface between the smoke ejected from the exit plane and the surroundings.

Figure 35 demonstrates that the profile in the exit plane is uniform except near its outer edge. A line joining the locations of the particles which most recently issued from the exit plane is indicative of the velocity distribution in the exit plane since the axial distance between a particular point and the exit plane is the product of the axial velocity at the corresponding radial station of the exit plane and a time increment. The slight rounding of the velocity profile near the outer edge of the exit plane is partly due to the way the velocity is computed numerically in the exit plane.

The fluid elements which emerged from the exit plane first are already showing the effect of the momentum exchange between the relatively high speed flow near the edge of the jet and the surroundings. The particles of smoke are rotating and moving radially into the flow field.

Figure 36 shows that the ring is already forming very quickly. Although the flow in the exit plane is still accelerating, the smoke has 


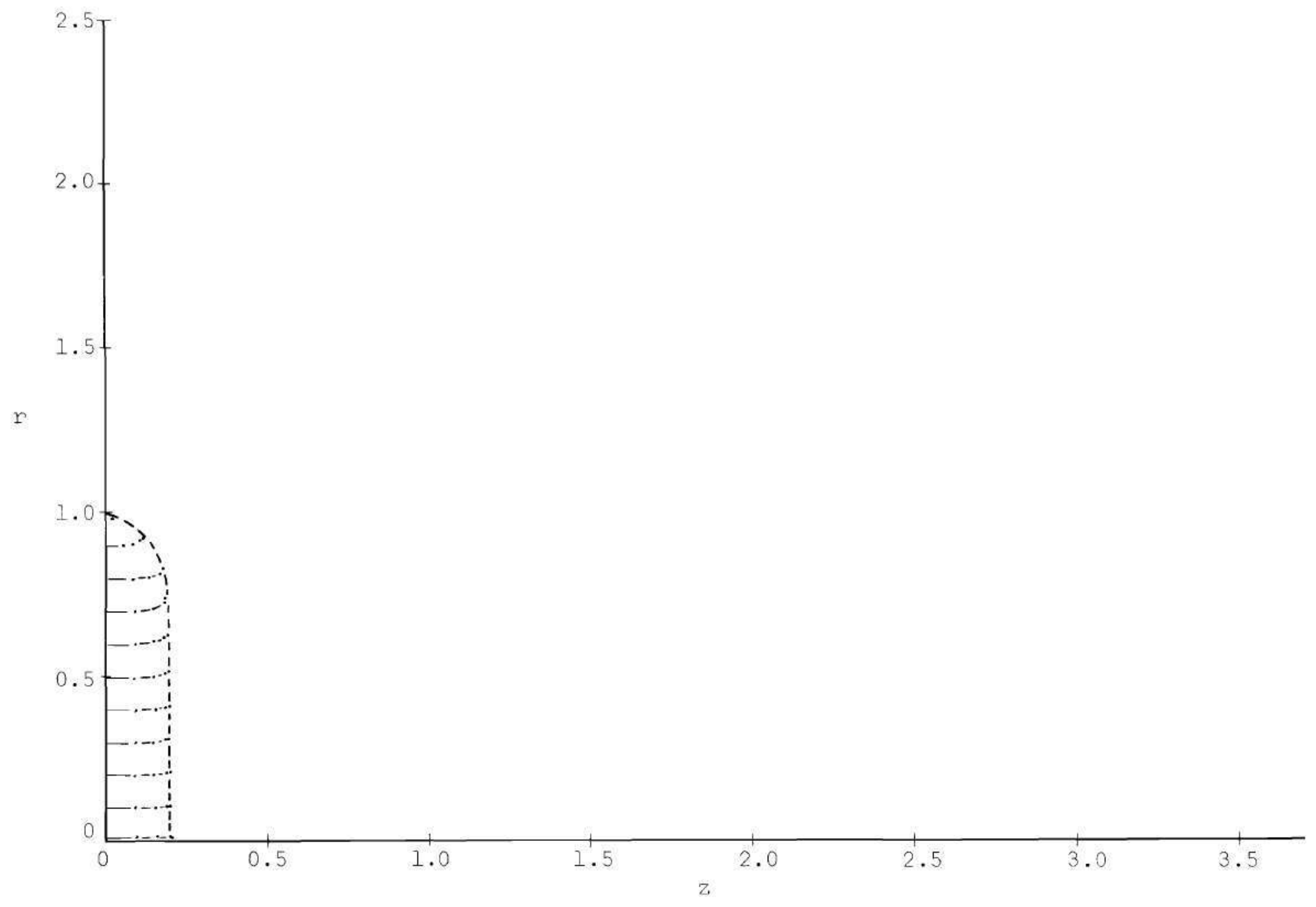

Figure 35. Streakline Pattern at $t=2$-- Uniform Profile 


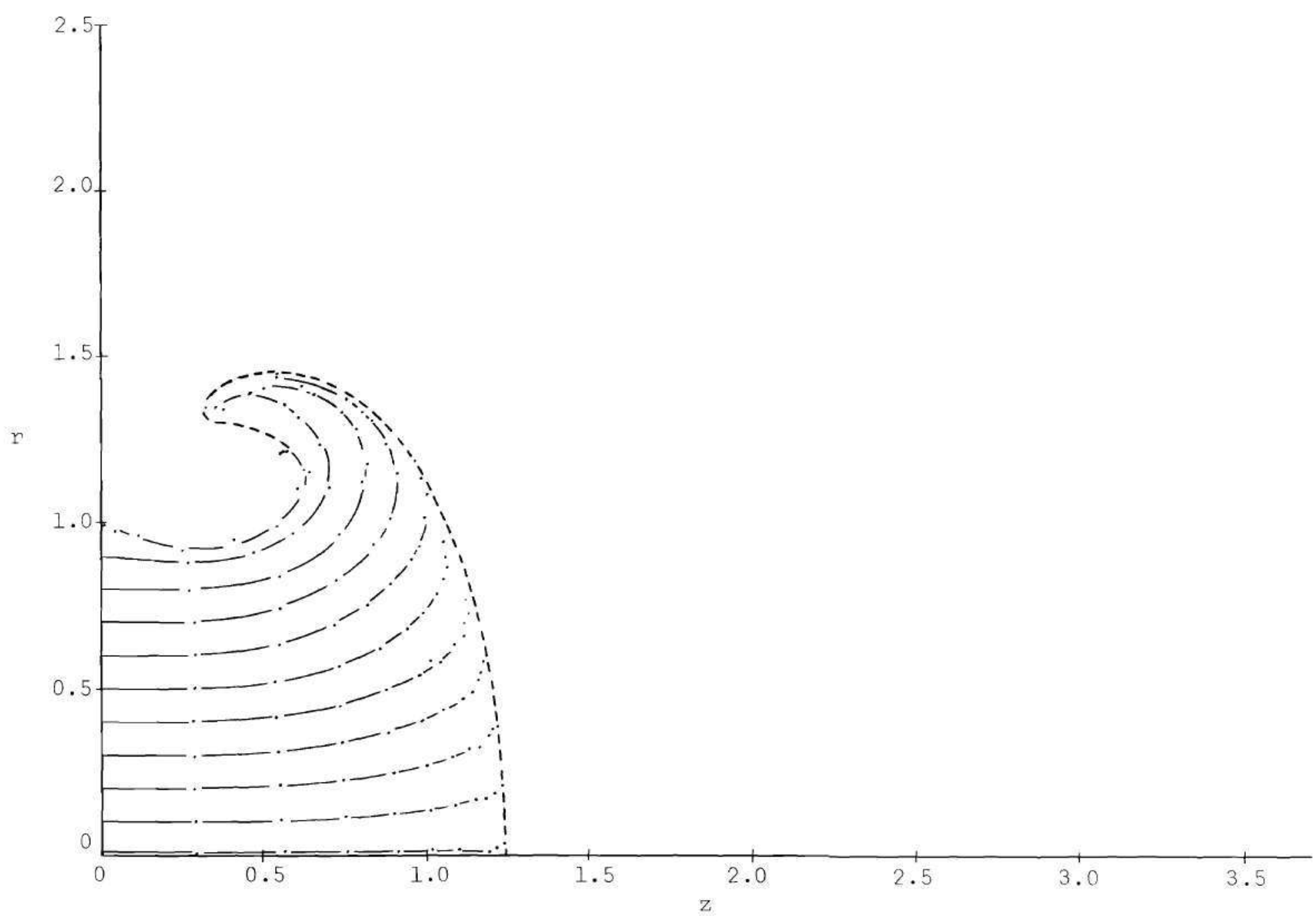

Figure 36. Streakline Pattern at $t=6$-- Uniform Profile 


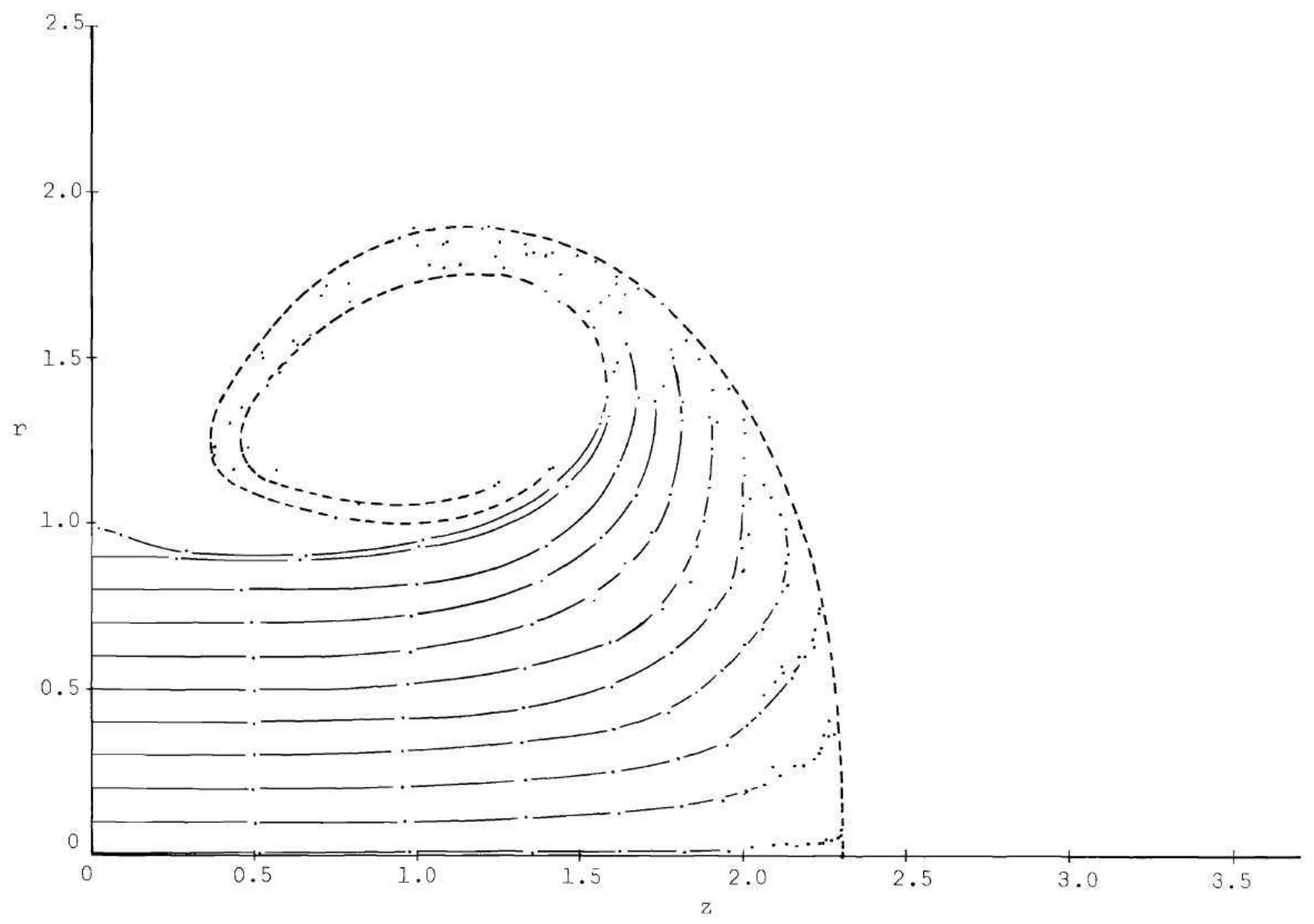

Figure 37. Streakline Pattern at $t=10--$ Uniform Profile 


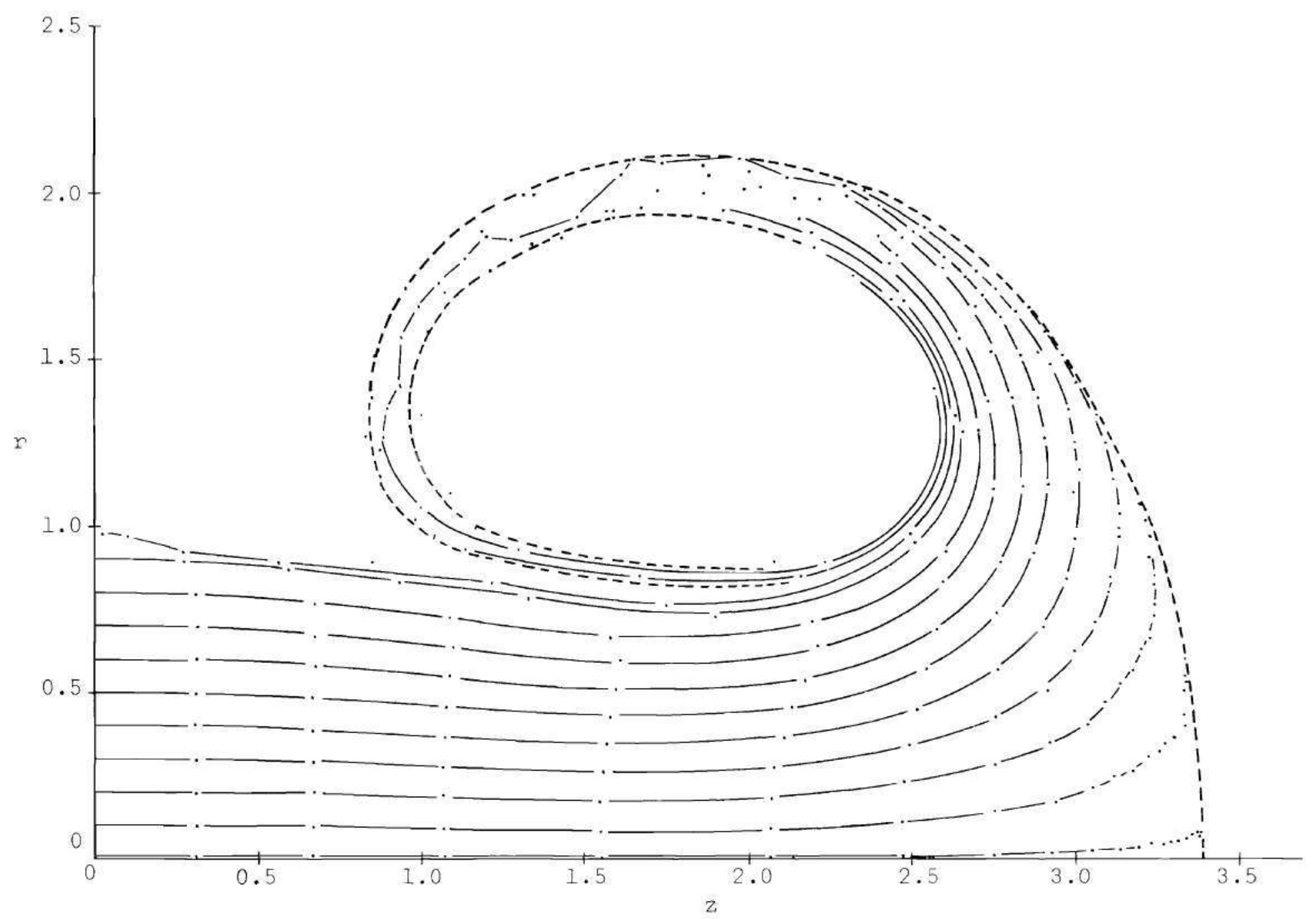

Figure 38. Streakline Pattern at $t=14--$ Uniform Profile 
already traveled about half way around the apparent center of the vortex. The same behavior which was discussed in the parabolic profile case is in evidence here. The smoke at the edge of the jet is rotating counter-clockwise as it is moving in the radial direction, and the air near the wall is moving into the jet. Toward the center of the jet, the smoke is moving axially. The mushroom shape of Figure 1 is beginning to form.

Figure 37 presents the streaklines at the time when the velocity distribution in the exit plane is at its maximum value. Although some portions of the streaklines are omitted at the top of the vortex, they are continued elsewhere to demonstrate that the streaklines which have their origins at large radial stations of the exit plane have already completely encircled the center of the ring. On a particular streakline, the greater distances between fluid elements near the exit plane indicate that these are high velocity elements. At the other end of some of these streaklines which encircle the vortex, the distance between fluid elements also indicates that these particles are moving rapidly. They have been in the flow field long enough to gain a large amount of momentum from the smoke which has since issued from the orifice. At the time of Figure 37, the vortex ring of the uniform profile has moved downstream almost twice as far as the one generated by the parabolic profile. This observation will be confirmed later in the discussion of the vorticity contours. It should also be noted in Figure 37 that the diameter of the jet decreases with increasing distance from the exit plane although the flow there has not yet started to decelerate. This too is evidence of the movement of fluid from the region near the wall 
into the jet. The minimum diameter of the jet of Figure 37 is somewhat smaller than that of the corresponding parabolic profile; this also reflects higher momentum possessed by the uniform jet.

Figure 38 illustrates the last of this series of streakline pictures. Soon, the ring will pass beyond the field of view afforded by the scale of the figure. The flow from the orifice is decelerating now, and the "necking down" phenomenon of Figure l-c is even more obvious. The distance between the fluid elements in the field indicates that the highest velocity flow now appears embedded in the flow field and not in the exit plane. It may appear that the center of the vortex ring is growing, but it is pointed out here that the portions of the streaklines have been omitted which correspond to the locations of elements which first came from the exit plane. However, it is the opinion of the author that if these were located more accurately with a finer spatial mesh and a smaller time increment, the resulting streaklines would spiral in toward the center of the vortex. This is the behavior which has been seen experimentally and which has been reported previously.

The effect of these numerical inaccuracies can be seen in one of the streaklines which is shown by the broken line of Figure 38 . It is emphasized that these streaklines represent numerical inaccuracies and not a physical phenomenon.

It is concluded from the streaklines of the uniform profile case that the numerical model has again produced a vortex ring.

A comparison of the streaklines of the parabolic profile (Eigures 9 through 14) and of the uniform profile (Figures 35 through 38) shows 
that the latter vortex is forming faster than the former one. The vortex ring resulting from the uniform profile is also larger than that of the parabolic profile. This difference can probably be attributed to a number of reasons. First, the mass flow and energy input to the system is higher for the uniform profile case than the parabolic profile case. Second, the contribution to the vorticity in the exit plane by the $\frac{\partial v_{z}}{\partial r}$ - component is concentrated at the edge of the exit plane for the uniform profile case. This is reflected by higher vorticities and rotational velocities near the edge of the jet, and these factors plus the higher translational velocity near the edge of the jet probably produce a larger ring for the uniform profile case. The Velocity Vector Field

Figure 39 illustrates a typical velocity vector plot for the uniform profile over a portion of the $z, r$ plane at the time $t=8$. Here, the velocity over the greater part of the exit plane has the value $v_{z}=0.8$. The velocity vectors indicate the same characteristics shown in the similar plot of the parabolic profile. Although a vortex is indicated in this figure, its center does not coincide with the center of the streakline pattern, because the velocity field includes the velocity of the vortex. However, if the translational velocity of the vortex is subtracted from the vector field, the center of the resulting plot will correspond to the center of the streakline pattern. The vector field shows air from the surroundings moving toward the jet, and the resulting velocity gradient normal to the wall may also be seen. The corresponding streamline distribution is shown in Figure 40. Once again the three-dimensionality of the problem somewhat 


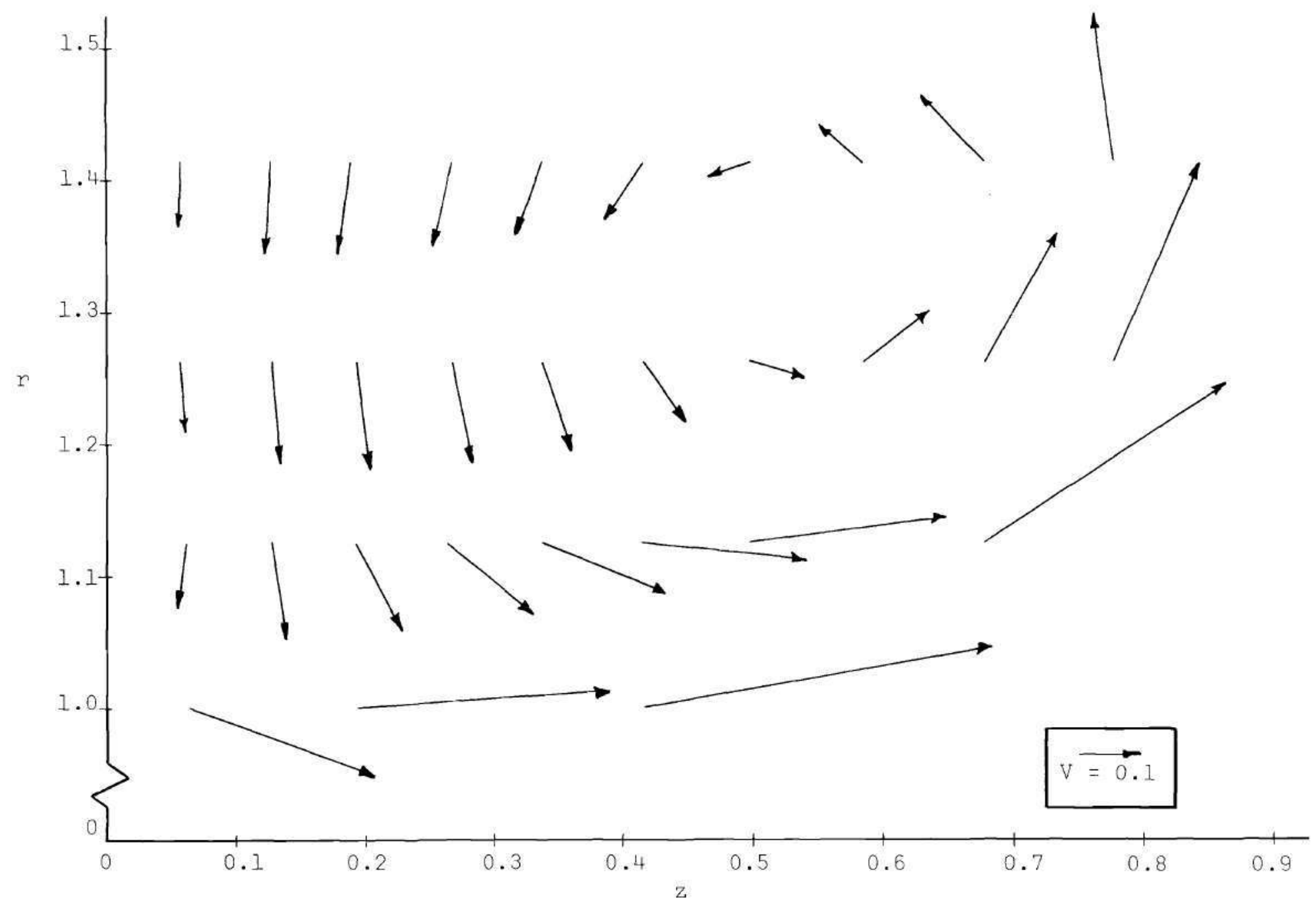

Figure 39. Portion of Velocity Vector Field at $t=8$-- Uniform Profile 


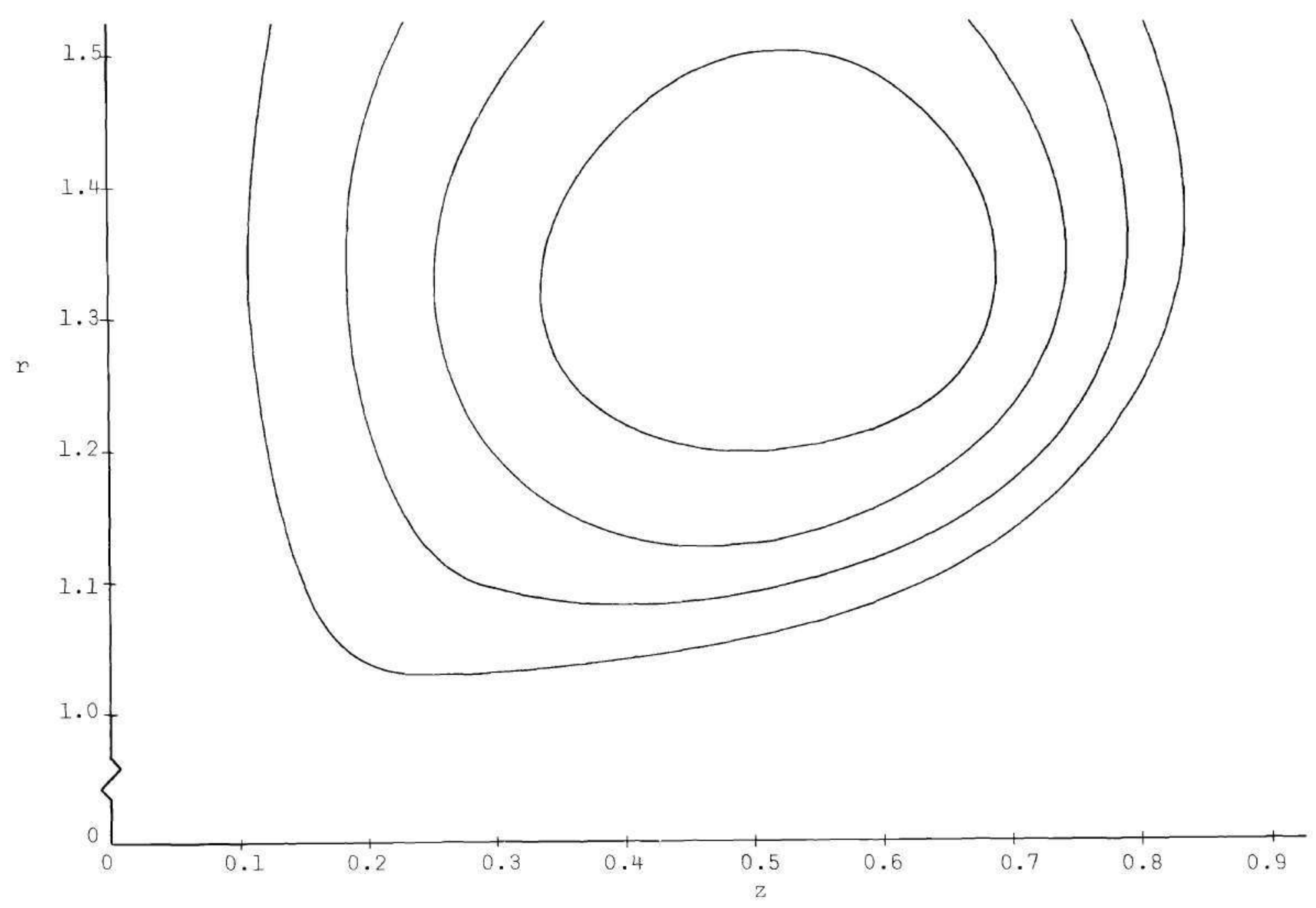

Figure 40. Portion of Streamline Pattern at $t=8--$ Uniform Profile 
disguises the very large velocity gradients. A slight divergence of adjacent streamlines indicates a large decrease in the velocity. Nevertheless, the instantaneous streamline pattern adequately describes all the phenomena shown by the velocity vectors. In addition, the velocity directions are indicated by the streamlines in a more continuous manner than in any other type of plot. The Vorticity Distributions and the Streamline Patterns In the following pages the instantaneous vorticity contours and the streamline patterns are presented in the form used for the parabolic profile case. Because a somewhat different behavior is indicated by the vortex ring generated by the uniform profile, an introductory plot of the vorticity distribution is given in Figure 41 at the time $t=12.0$ over a small portion of the $z, r$ plane. The characteristic which is immediately noticed in this illustration is the presence of small regions of negative vorticity in that part of the $z, r$ plane for which $r<1$. It is mentioned in Appendix $H$ that although these islands of negative vorticity appear in both the test case for $M=16$ and the test case for $M=24$, their locations do not always compare well with each other. This indicates that convergence to within a high degree of accuracy has not yet been achieved in this portion of the $z, r$ plane. Nevertheless, a phenomenon is occurring which should be explained. Consider the vorticity on the boundary $z=0$ as it is expressed by its definition.

$$
\omega=\frac{\partial v_{r}}{\partial z}-\frac{\partial v_{z}}{\partial r}
$$




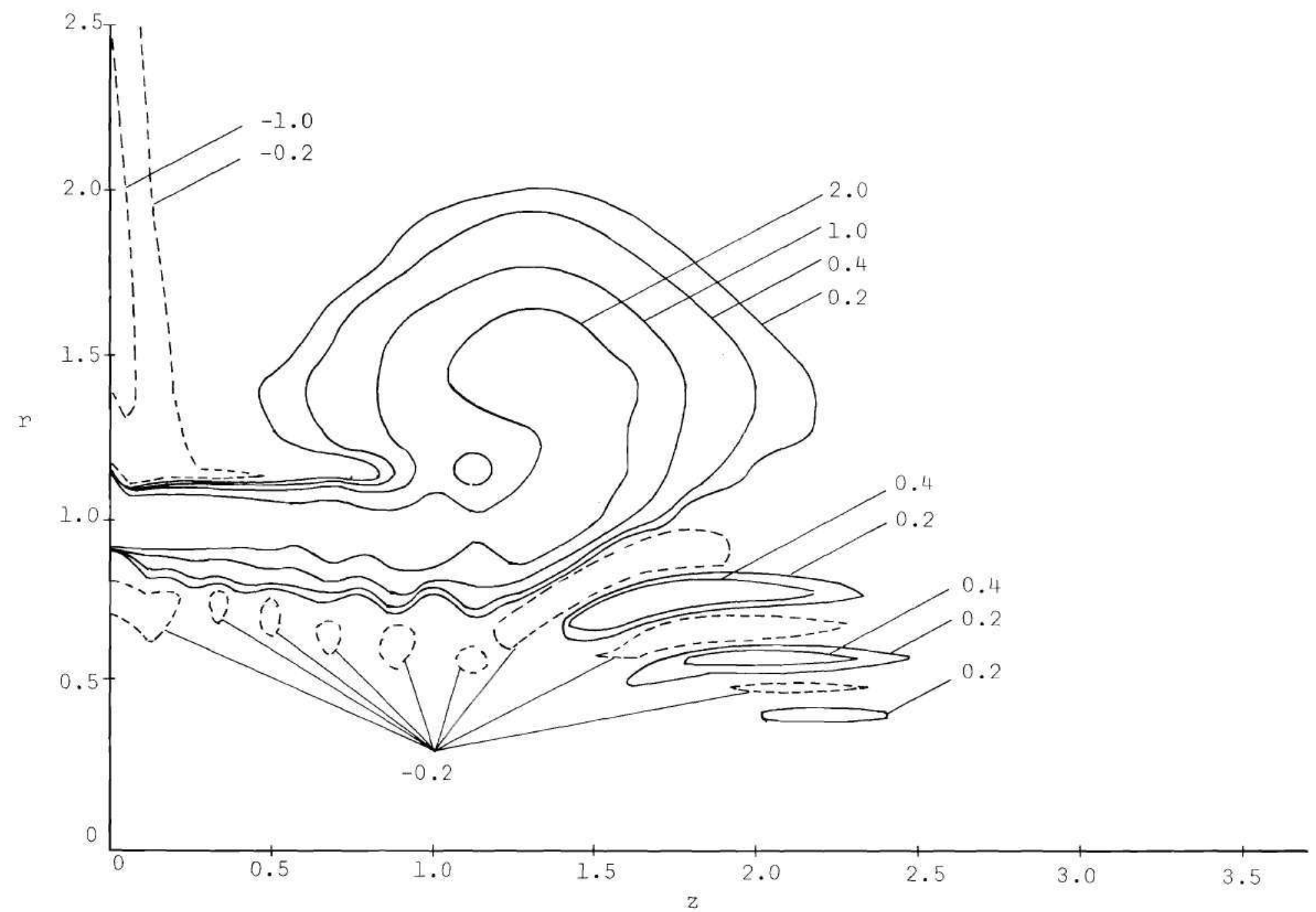

Figure 41. Vorticity Distribution at $t=12$-- Uniform Profile 
The second term of this expression, the radial derivative of the axial velocity, is everywhere zero except at the edge of the orifice which corresponds to $r=1$ at the mesh point $j=P$. This component vanishes for $j<P$ because the velocity profile in the exit plane is uniform and for $j>P$ because the axial velocity is zero on the solid boundary. If the velocity profile were completely uniform out to $r=1$, the vorticity at this point would be infinite. However, this is an unrealistic physical situation, so the profile has been adjusted with a sharp linear gradient at the edge of the orifice. (This is shown in Figune 7.) Thus the derivative $\frac{\partial v}{\partial r}$ is large but finite at the mesh point $j=P$. Since this term has a negative value, the contribution to the vorticity is positive.

Consider now the other component of vorticity, the axial derivative of the radial velocity, $\frac{\partial v_{r}}{\partial z}$. After a few initial time steps of the numerical solution, air moves from the surroundings towards the jet until it is finally turned in the axial direction due to the interaction with the smoke issuing from the exit plane. Since the radial velocity is zero on the boundary and then negative a short positive axial distance away, the resulting derivative, $\frac{\partial v_{r}}{\partial z}$, is necessarily negative. This in turn creates a negative contribution to the vorticity on the solid wall. Since there is no other component here, the vorticity on the wall is negative. This was also the case for the parabolic profile, and this behavior has been discussed with that case. However, in the exit plane the vorticities in the two cases are somewhat different.

Downstream of the exit plane, the streaklines indicate a negative radial velocity over the outer portion of the jet. Since the radial 
velocity in the exit plane is specified as zero, the derivative $\frac{\partial v_{r}}{\partial z}$ is necessarily negative over a portion of the exit plane. For the uniform profile, this is the only contribution to the vorticity, and the vorticity in the exit plane is negative. On the other hand, the parabolic profile offers a contrasting behavior. Again, since the radial velocity is specified as zero in the exit plane, the negative radial velocity downstream of the exit causes a negative axial derivative of the radial velocity in the exit plane. However, this term is not the only contributing factor to the vorticity. Although the derivative $\frac{\partial v_{r}}{\partial z}$ is of the same order of magnitude as the derivative $\frac{\partial v_{z}}{\partial r}$, the former term does not dominate the latter, and the resulting difference of these quantities, i.e., the vorticity, is positive. This explains why the vorticity in the exit plane in the parabolic case is positive whereas the vorticity in the uniform case is negative. Both cases experience a negative value for the derivative $\frac{\partial v_{r}}{\partial z}$, but the parabolic case also possesses an additional contribution which maintains a positive vorticity in the exit plane. The results of both cases are consistent within the specifications of the numerical model.

Now the mechanism by which these regions of negative vorticity are distributed throughout the flow field must be examined. After a certain period of time during which the velocity in the exit plane is continuously increasing, the flow along the wall reflects a negative radial velocity downstream of most of the exit plane. The sign of this radial velocity changes near the center line where the flow is still expanding radially. The negative radial velocity corresponds to a negative vorticity which moves downstream with the fluid elements. 
Thus, a large radial gradient in the vorticity exists between the region of high positive vorticity near the edge of the exit plane and the region of smaller negative vorticity which is generated in the exit plane. These vorticity regions may be seen in Figure 41 . Vorticity transfer in the radial direction is associated with this vorticity gradient. The effect is more evident in the regions of negative vorticity where the value is not high initially. The large area of negative vorticity breaks up into smaller ones, and these are swept downstream by the momentum of the fluid. As time progresses, the action of viscosity steadily diminishes the magnitude of the negative vorticity.

Although these small annuli of negative vorticity are predicted by both of the cases studied (i.e., $M=16$ and $M=24$ ), the results of the two cases do not reproduce exactly over a portion of the flow field. Evidently, this disagreement is a problem of convergence which can be solved by using a finer mesh size. Since a representative picture of these regions of negative vorticity has been given in Figure 4l, the generation and breakup of these small regions of negative vorticity will not be given in detail in the following figures for clarity. The vorticity distributions and streamline patterns for the uniform profile case are presented in Figures 42 through 48 for equal time increments of $\delta t=4$ starting at $t=4$ and ending at $t=28$. The values of the vorticity plotted are shown on the figures. Again, the streamlines emerging from the exit plane are plotted for convenient values of the radius $r$ in order to present a detailed description. Recalling that a stream channel is defined as the region between adjacent streamlines, it should be noted that the mass flows of adjacent stream channels 


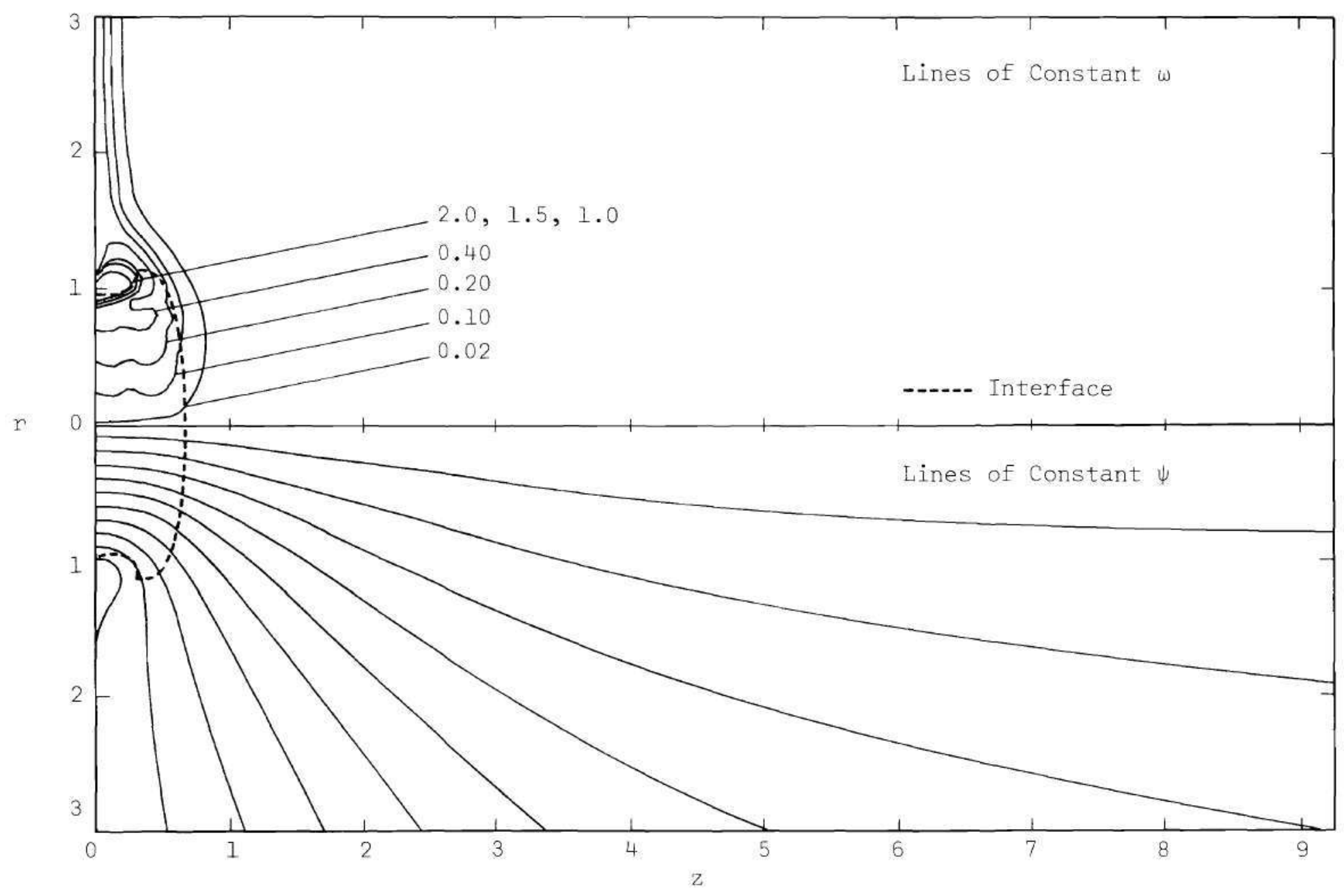

Figure 42. Vorticity Distribution and Streamline Pattern at $t=4$-- Uniform Profile 


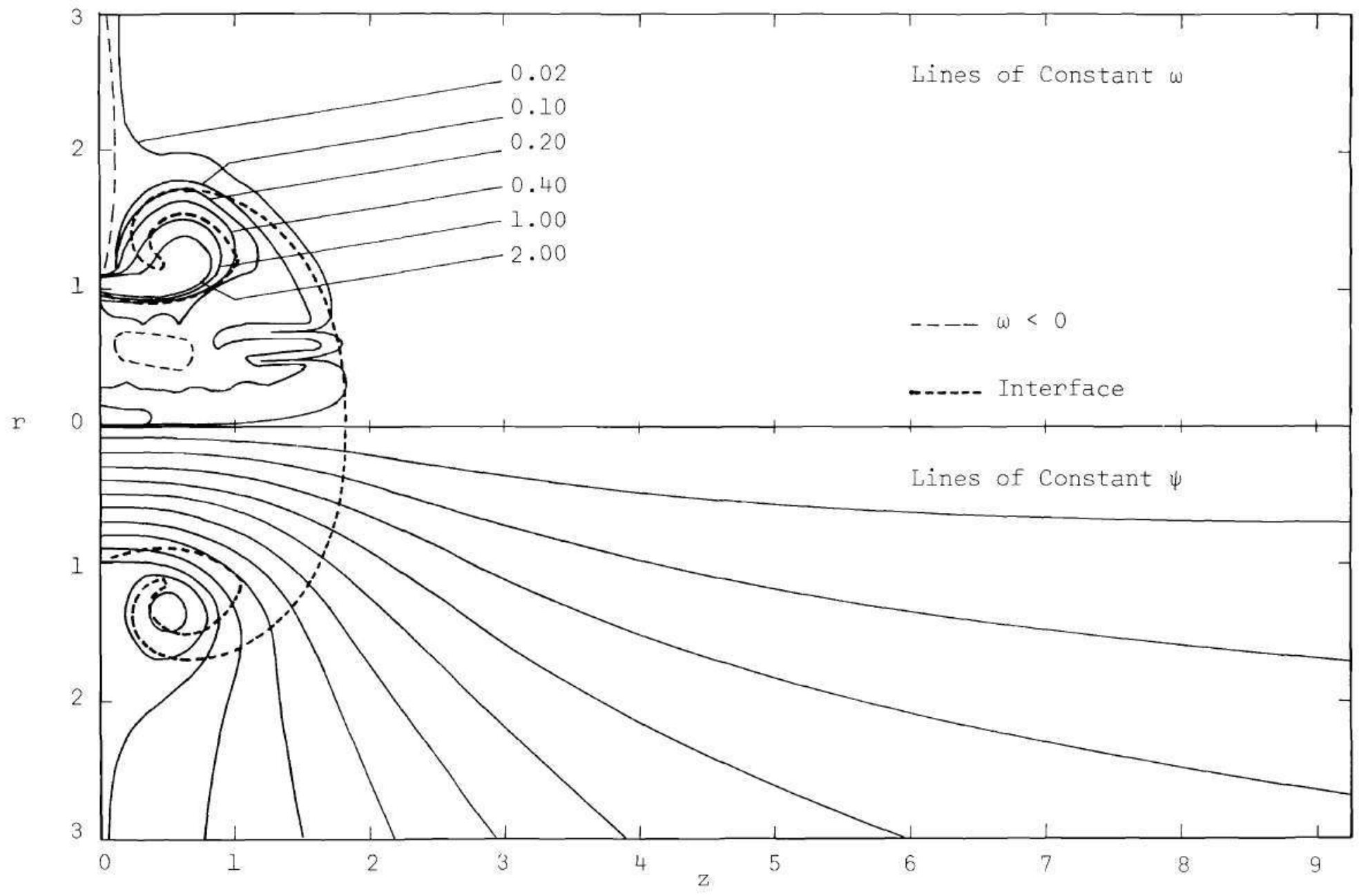

Figure 43. Vorticity Distribution and Streamline Pattern at $t=8$-- Uniform Profile 


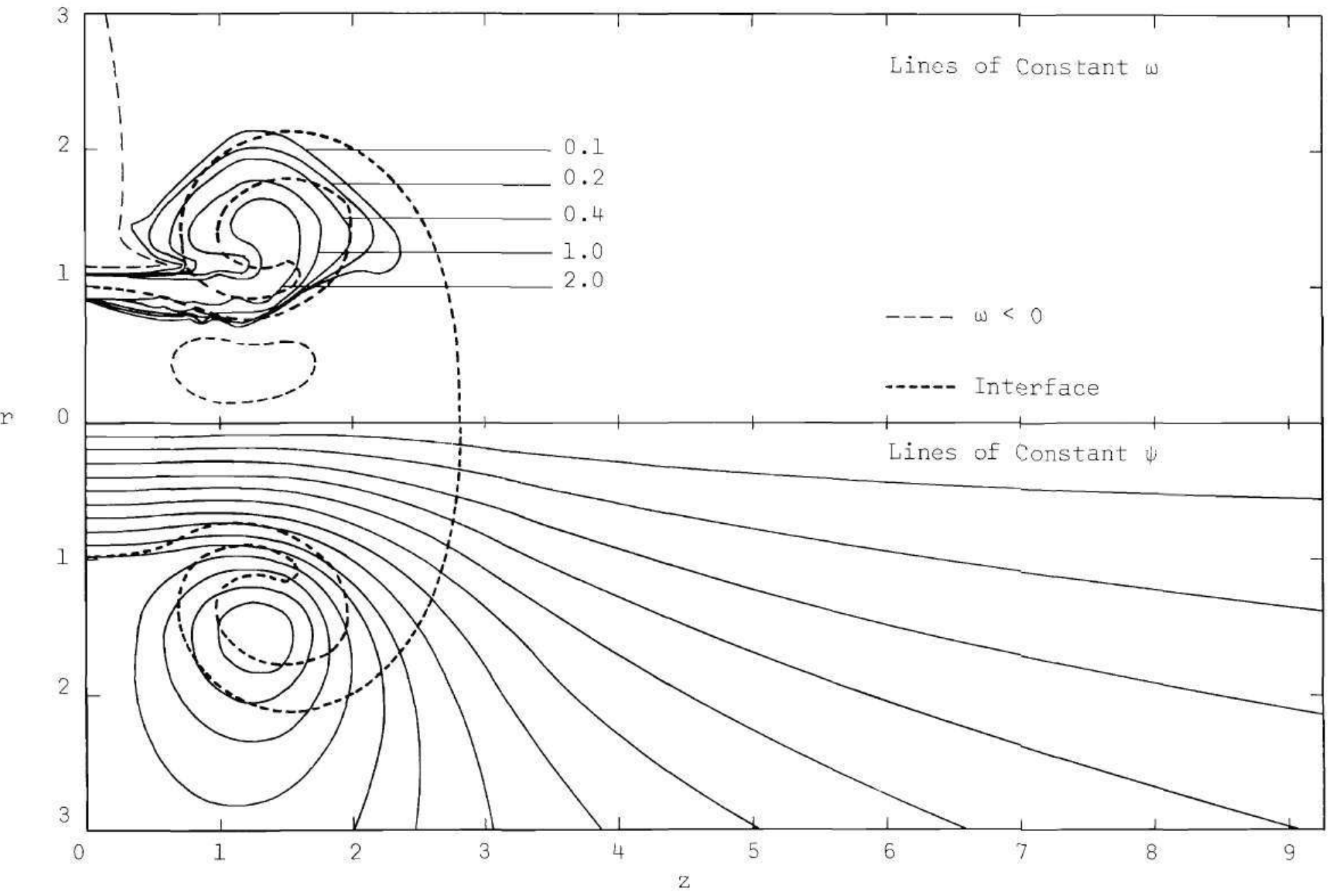

Figure 44. Vorticity Distribution and Streamline Pattern at $t=12$-- Uniform Profile 


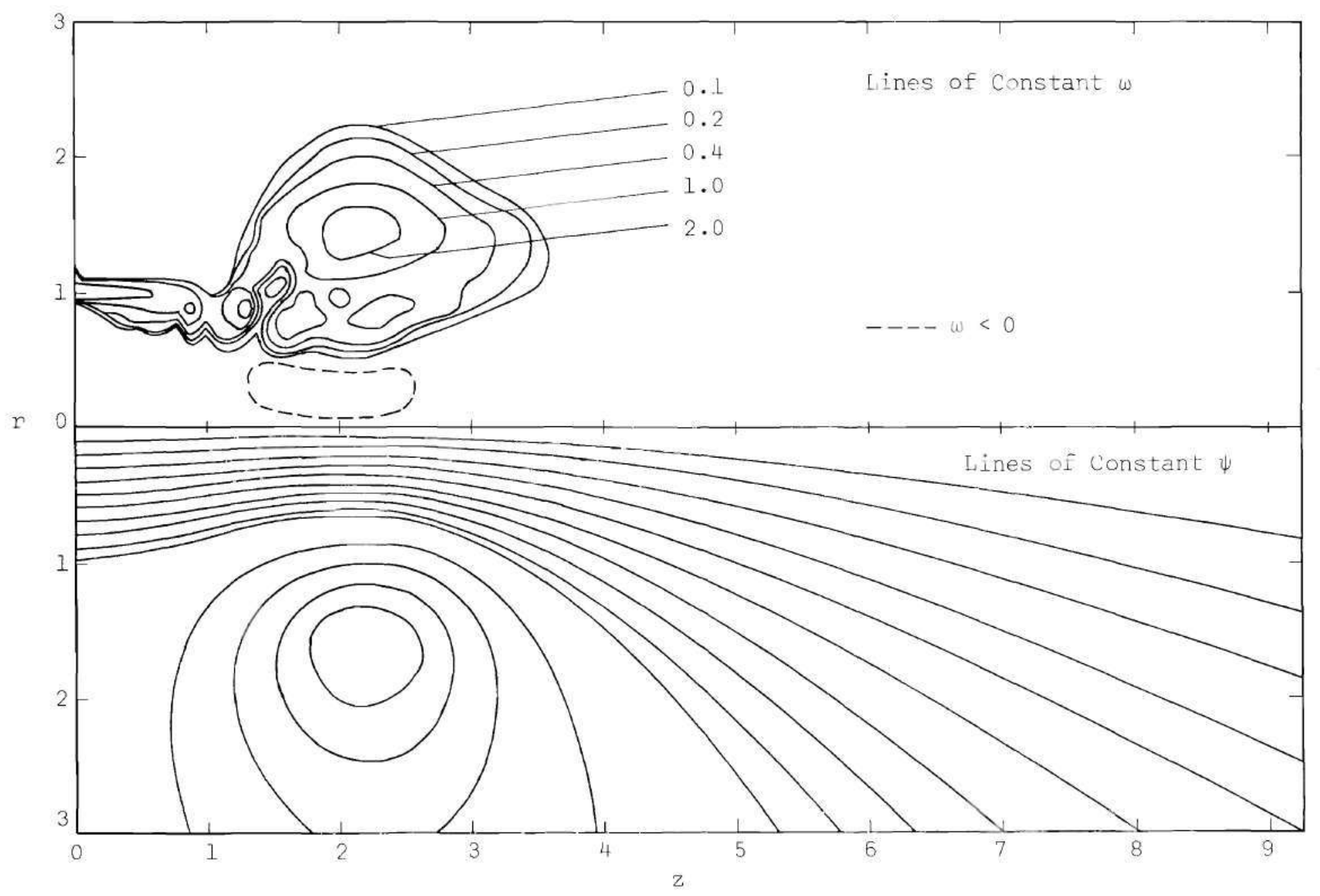

Figure 45. Vorticity Distribution and Streamline Pattern at $t=16$-- Uniform Profile 


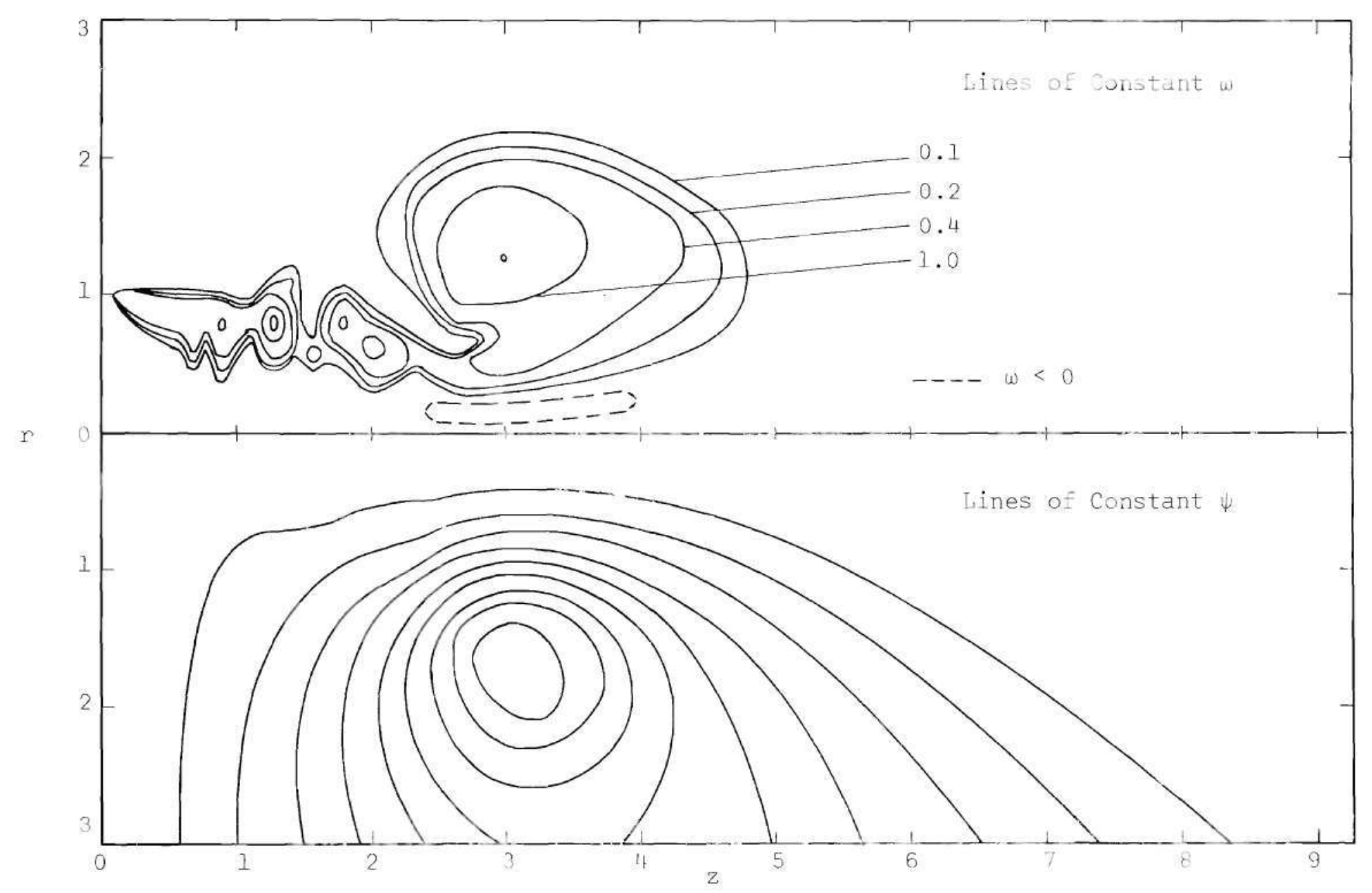

Figure 46. Vorticity Distribution and Streamline Pattern at $t=20--$ Uniform Frofile 


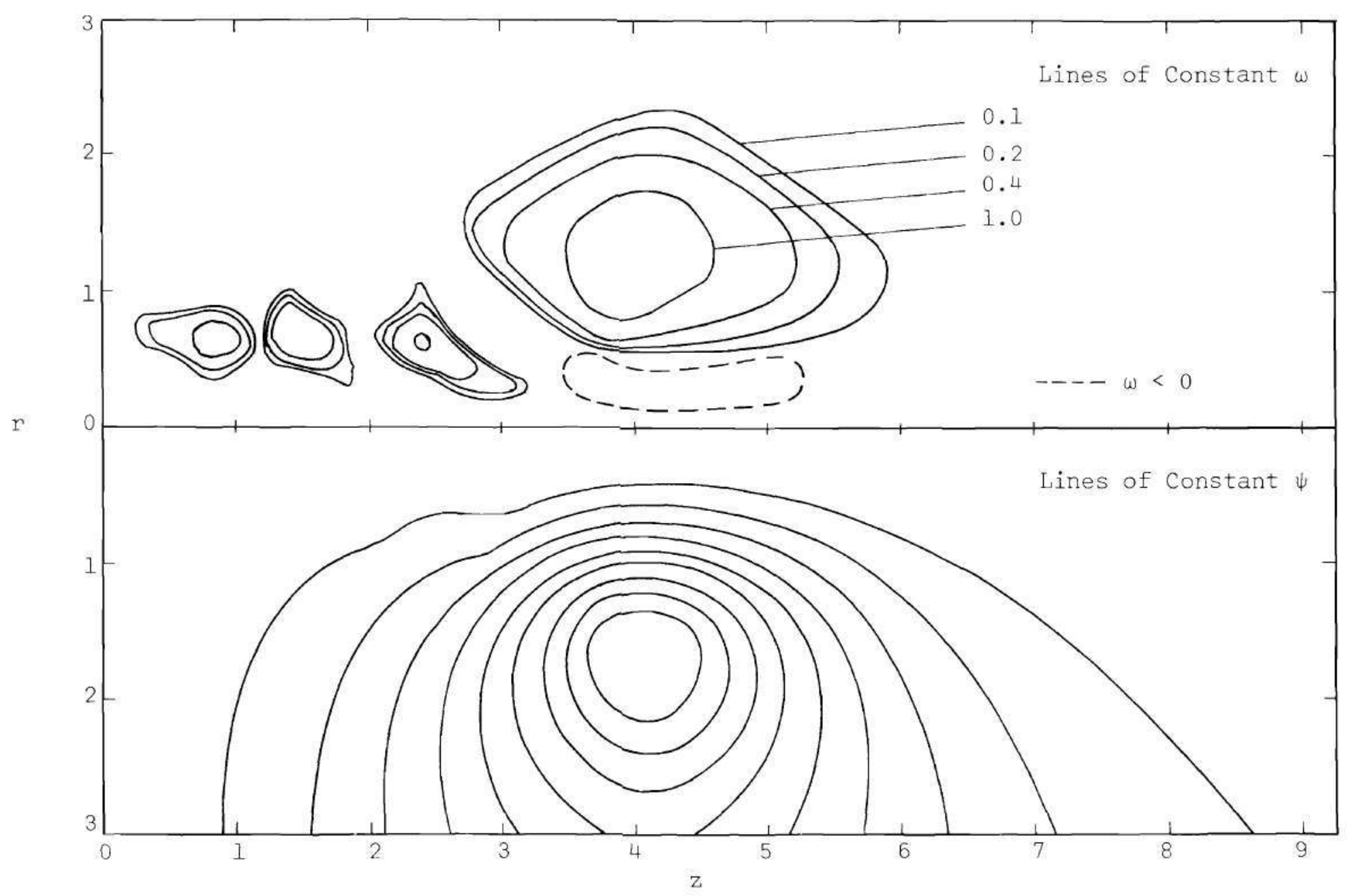

Figure 47. Vorticity Distribution and Streamline Pattern at $t=24$-- Uniform Profile 


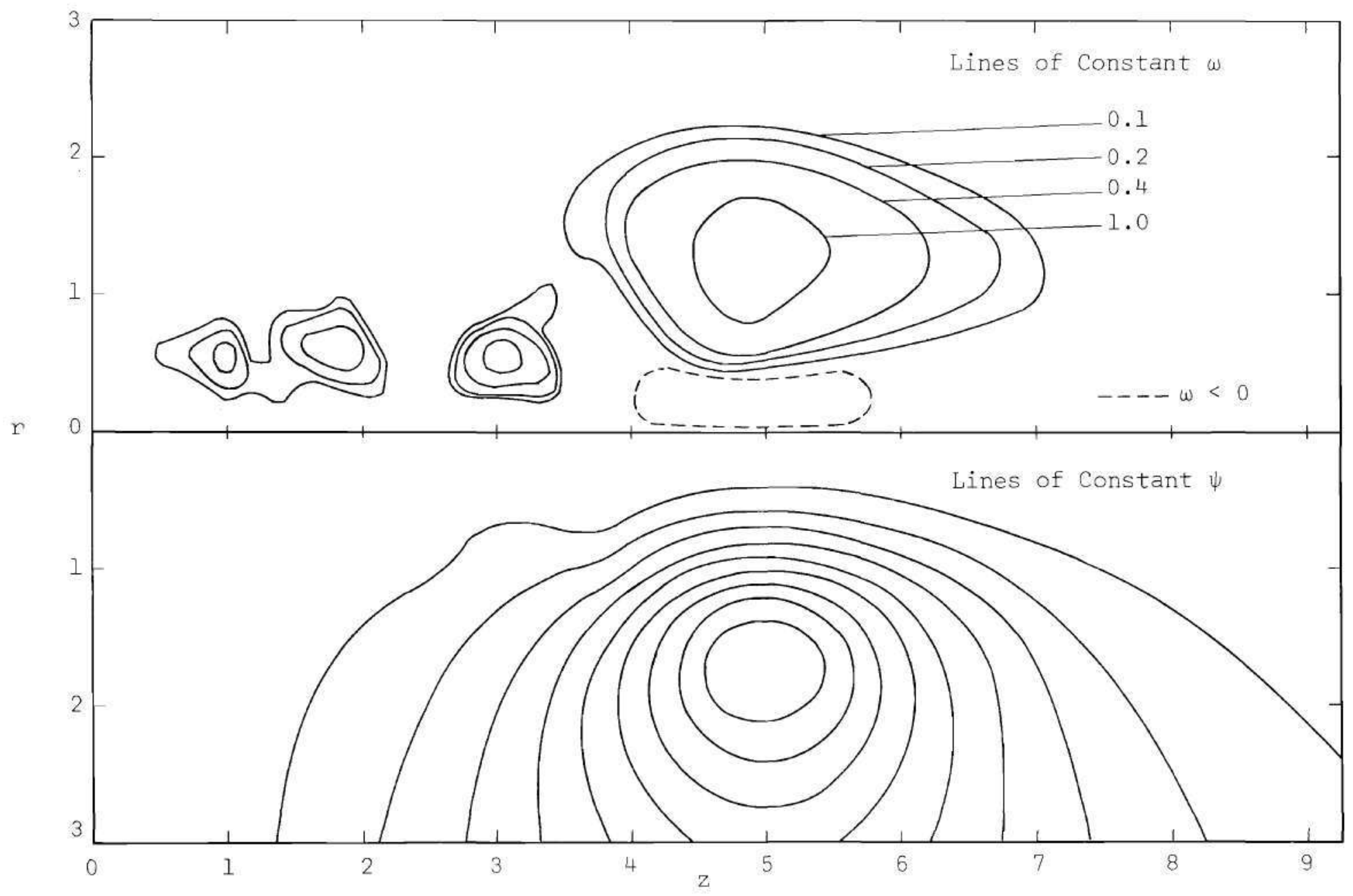

Figure 48. Vorticity Distribution and Streamline Pattern at $t=28$-- Uniform Profile 
Table 4. Values of the Stream Function for the Plotted Streamlines--Uniform Profile Case

\begin{tabular}{|c|c|c|c|c|c|c|c|}
\hline Index & $t=4.0$ & $t=8.0$ & $t=12.0$ & $t=16.0$ & $t=20.0$ & $t=24.0$ & $t=28.0$ \\
\hline 1 & $2.00(-3)^{*}$ & $4.00(-3)$ & $4.00(-3)$ & $2.00(-3)$ & 7. $36(-2)$ & $6.71(-2)$ & $6.50(-2)$ \\
\hline 2 & $8.00(-3)$ & $1.60(-2)$ & $1.60(-2)$ & $8.00(-3)$ & $1.47(-1)$ & $1.34(-1)$ & $1.30(-1)$ \\
\hline 3 & $1.80(-2)$ & $3.60(-2)$ & $3.60(-2)$ & $1.80(-2)$ & $2.2 I(-1)$ & $2.01(-1)$ & $1.95(-1)$ \\
\hline 4 & $3.20(-2)$ & $6.40(-2)$ & $6.40(-2)$ & $3.20(-2)$ & $2.94(-1)$ & $2.68(-1)$ & $2.60(-1)$ \\
\hline 5 & $5.00(-2)$ & $1.00(-1)$ & $1.00(-1)$ & $5.00(-2)$ & $3.68(-1)$ & $3.35(-1)$ & $3.25(-1)$ \\
\hline 6 & $7.20(-2)$ & $1.44(-1)$ & $1.44(-1)$ & $7.20(-2)$ & $4 \cdot 42(-1)$ & $4.03(-1)$ & $3.90(-1)$ \\
\hline 7 & $9.80(-2)$ & $1.96(-1)$ & $1.96(-1)$ & $9.80(-2)$ & $5.15(-1)$ & $4.70(-1)$ & $4.55(-1)$ \\
\hline 8 & $1.28(-1)$ & $2.56(-1)$ & $2.56(-1)$ & $1.28(-1)$ & $5.89(-1)$ & $5.37(-1)$ & $5.20(-1)$ \\
\hline 9 & $1.62(-1)$ & $3.24(-1)$ & $3.24(-1)$ & $1.62(-1)$ & $6.62(-1)$ & $6.04(-1)$ & $5.85(-1)$ \\
\hline 10 & $1.90(-1)$ & $3.80(-1)$ & $3.80(-1)$ & $1.90(-1)$ & & & \\
\hline 11 & & & $4.54(-1)$ & $3.08(-1)$ & & & \\
\hline 12 & & $4.13(-1)$ & $5.28(-1)$ & $4.25(-1)$ & & & \\
\hline 13 & & & $6.02(-1)$ & $5.42(-1)$ & & & \\
\hline 14 & & $4.46(-1)$ & $6.76(-1)$ & $6.60(-1)$ & & & \\
\hline
\end{tabular}

$* 2.00(-3)=2.00 \times 10^{-3}$ 
emerging from the exit are not equal. However, the streamlines which do not emanate from the exit plane are plotted for equal increments of the stream function, and for these streamlines adjacent stream channels do indicate equal mass flow rates. The value of the stream function for each streamline is given in Table 4 immediately after the figures.

Figure 42 illustrates the vorticity contours and the streamlines shortly after the flow in the exit plane begins. No negative radial velocity has yet appeared downstream of the exit plane although a region of negative velocity does occur along the solid boundary near the edge of the orifice. This behavior is indicated by positive vorticity over the exit plane and a region of negative vorticity near the solid boundary which itself is embedded in a region of positive vorticity. The streamlines show fluid emerging from the exit and expanding around the corner of the orifice in the radial direction, and the streamline which returns to the wall encloses the fluid which will become the center of the streamline pattern of the vortex.

In Figure 43, the vortex ring is beginning to form. A large area of very high vorticity corresponds roughly to the center of the ring. The value of this vorticity is about twice as high as that found at any time in the parabolic profile case. This is due to the gradient in the axial velocity profile in the exit plane near the edge of the orifice. The dashed contour downstream of the exit plane indicates the portion of the flow field where the breakup of the region of negative vorticity is occurring. This portion of the flow field is too complex to plot in detail. Near the center line, there exists a region of positive vorticity which reflects the radial expansion of the smoke from the exit plane. 
The streamline pattern illustrates that the formation of the ring is well advanced even at this early time. The high vorticity occurs concomitantly with a high value of the stream function over a large area, and this results in a distribution of closed streamlines over a fairly large region of the flow. Comparison of the streamlines for the uniform and parabolic profile cases at the same time (i.e., comparison of Figure 42 and Figure 16) shows that the formation of the ring is more advanced for the uniform profile than the parabolic profile since more mass and energy are involved in the former case than the latter.

The value of the velocity in the exit plane has reached its peak and is now decreasing in Figure 44. This is evidenced by the slight converging of the streamlines between the vortex center and the center line. A negative radial velocity occurs outside the exit plane, and in this region negative vorticity is generated by the negative velocity. The space between the center line and the positive vorticity contours is occupied by regions of positive and negative vorticity corresponding to the breakup of the larger regions of negative vorticity. These small swirls are also causing the waviness of the vorticity contours in the region around $r=1$. The enlarging of the area of the closed streamlines indicates that more and more fluid is being drawn into the swirl of the vortex.

For Figure 45 the velocity in the exit plane has almost ceased. The annulus of high vorticity fluid extending from the boundary to the vortex is beginning to break apart. The large region of positive vorticity which indicates the vortex ring has spread to include more of 
the fluid, and the viscous stresses have decreased. The converging of the streamlines between the center of the swirl and the center line indicates that the highest velocities in the field are no longer in the exit plane but in the region around the vortex. More air has moved into the swirl of the vortex.

Figure 46 gives a picture of the field at the moment when the velocity in the exit plane ceases. The high vorticity region of the vortex ring is separating from the fluid near the boundary. A smoke picture taken at this time would show the smoke between the orifice and the ring pinching off as in Figure l-c. The annulus of high vorticity fluid between the ring and the boundary is moving toward the center line as shown by this and the previous figures. This motion also indicates that negative radial velocities exist upstream of the ring near the center line. The irregularities in the streamline which is closest to the center line show that the breakup of the annulus of vorticity between the ring and the boundary is having an effect on the streamline pattern. Secondary vortices are being formed as the breakup occurs.

Figure 47 and Figure 48 give the vorticity distribution and streamline pattern after the flow in the exit plane has terminated. The vortex ring is continuing to move downstream. The trailing vortices are being left further and further behind with each succeeding time increment. One of them is separating from the others. It and the regions of negative vorticity on either side are appearing to cause a fairly large secondary rotational velocity as shown by the streamline closest to the center line. However, this effect is being felt over an 
increasingly smaller portion of the field. This is evidenced by the absence of irregularities in the other streamlines which surround the swirl.

Once again, no mention is made of the completion of the ring. Although Okabe and Inoue [30] claim that the ring is complete when a hole appears at the vortex center, it appears from these results that the formation and decay of a vortex ring is a continuous process. No clearly defined time occurs before which the ring is forming and after which the ring is decaying.

\section{Velocity Profiles}

Two plots are given in this section to show the behavior of certain portions of the flow field which may not have been evident from the illustrations of the instantaneous streaklines, vorticity contours, and streamlines. Figure 49 presents the axial velocity distribution as a function of the radial co-ordinate $r$ at the time $t=28.0$ and at an axial location in the flow which is close to the vortex center. It is immediately noticed that in this velocity profile (which is a typical one) the maximum velocity does not occur at the center line. However, a maximum velocity at the center line is the expected behavion for the vortex ring associated with the circular vortex filament of Figure 34. The difference between these two velocity profiles should be examined.

First, it should be mentioned that the positive value of the derivative $\frac{\partial v_{z}}{\partial r}$ in Figure 49 corresponds to the region of negative vorticity in Figure 48 at $z \cong 5$. For $r>0.5$, the term $\frac{\partial v}{\partial r}$ is negative, and the positive vorticity of Figure 48 for $z \cong 5$ and $r>0.5$ 


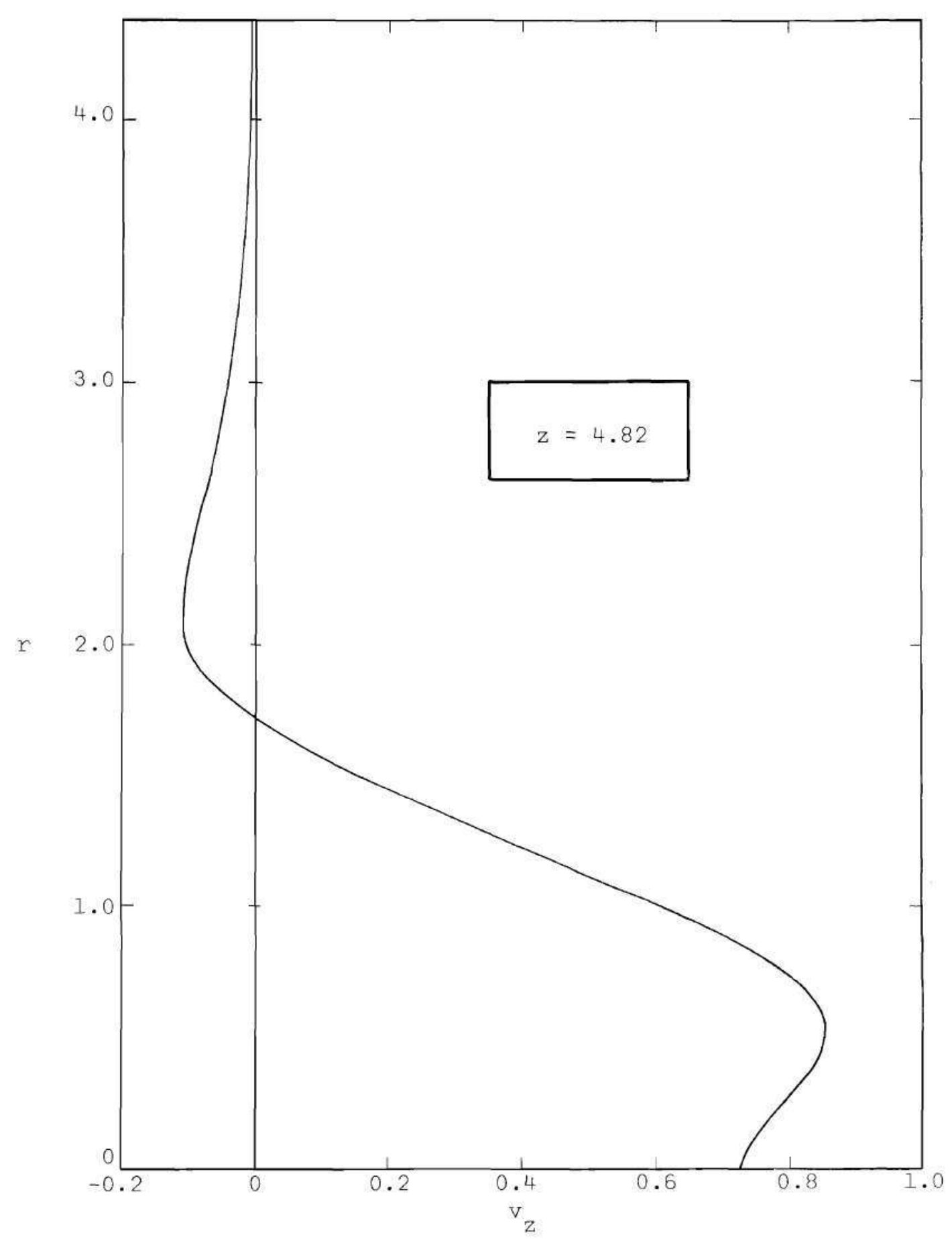

Figure 49. Axial Velocity Distribution near Vortex Center at $t=28$-- Uniform Profile 
reflects this behavior. The existence of a local maximum in the velocity profile may be explained physically by examining the flow at different times. In the initial stages of the vortex formation, smoke from the jet expands with a radial velocity component while air from the surroundings moves in toward the jet. The interaction between these two regions of fluid is shown by a local maximum in the axial velocity as illustrated in Figure 50-a. As time progresses, more fluid moves into the swirl of the vortex, and the action of viscosity begins to take effect near the center of the swirl. The maximum in the axial velocity profile moves radially inward. This is given in Figure 50-b. Finally at some later time (which was not reached in this numerical solution) the local maximum should move to the center line as shown in Figure 50-c. The time at which the local maximum in the axial velocity profile reaches the center line may be a convenient time to use in defining when the vortex is fully developed. In the case of the parabolic profile in which the velocity gradients were not quite so high, a local maximum in the velocity profile reflecting the merging of fluid near the edge of the exit plane did not occur.

Figure 51 illustrates a typical axial velocity distribution along the center line. This plot is similar to the corresponding figure for the parabolic profile case, and it is indicative of the large velocity gradients which occur near the vortex.

\section{Concluding Remarks}

The instantaneous streaklines, velocity vector fields, vorticity contours, and streamline patterns for both the parabolic profile case 

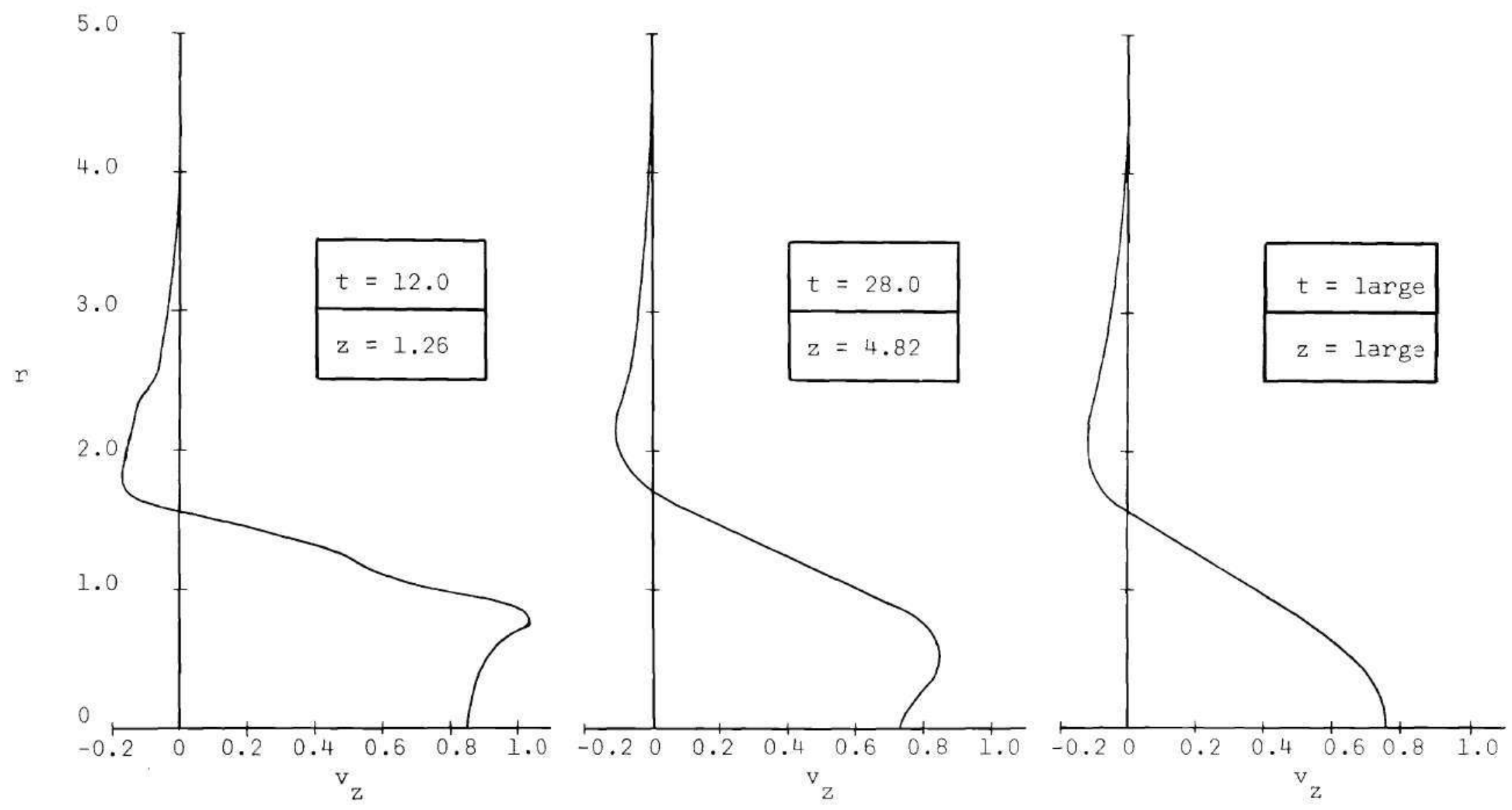

Figure 50. Axial Velocity Distributions Near Vortex Center at Various Times--Uniform Profile 


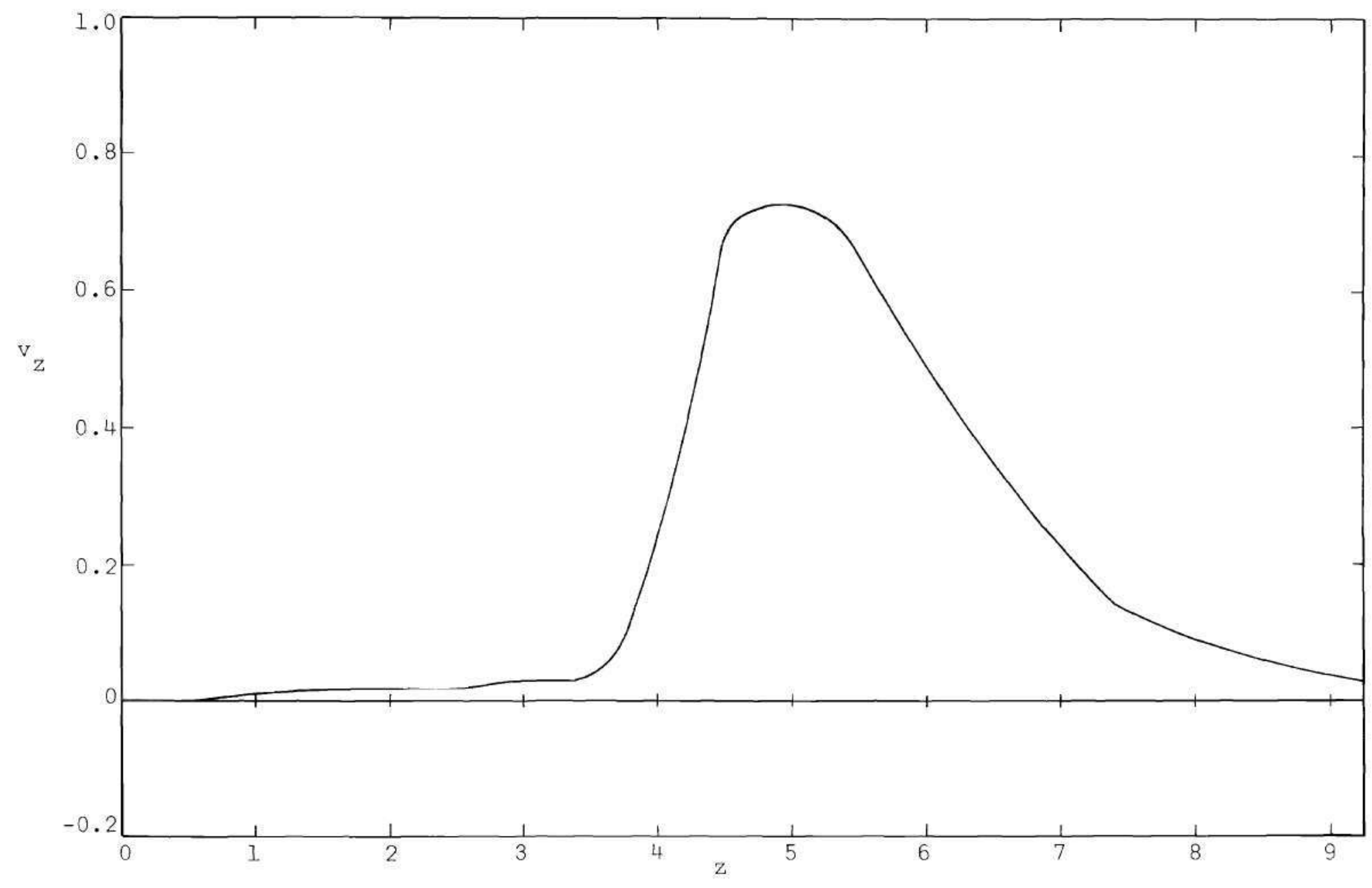

Figure 51. Axial Center Line Velocity at $t=28$-- Uniform Profile 
and the uniform profile case demonstrate the generation of a vortex ring. At this point, the similarities and differences between the results of the two cases will be quickly summarized.

The smoke which escapes the orifice expands both axially and radially into the flow field, and the velocity difference between the emerging fluid and the quiescent surroundings is shown by rotational velocity of the fluid elements. In addition, fluid is drawn into the jet from the region along the solid boundary, and this motion coupled with the rotational velocity of the fluid emanating from the orifice ultimately leads to the swirl of the vortex ring. It is difficult to precisely define a time at which the vortex ring is complete and at which the decay that leads to the breakup of the vortex commences. It appears that the behavior of the vortex is continuous, and no clearly defined periods of development and degeneration can be specified. Since more momentum is introduced into the flow by the uniform velocity profile than by the parabolic velocity profile, the resulting vortex ring develops faster, produces a larger ring, and exhibits higher values of vorticity for the former case than for the latter. There also exist qualitative differences in the flow field which are associated with the velocity profiles specified in the exit plane. Although these differences occur primarily in the vicinity of the exit plane, they may be noted elsewhere also. After the initial stages of the vortex formation, a negative radial velocity occurs outside the exit plane as shown by the convergence of the streaklines and streamlines. Hence, the $\frac{\partial v_{r}}{\partial z}$-component of vorticity in the exit plane is negative. For the parabolic profile case, the $\frac{\partial v}{\partial r}$-component of vor- 
ticity dominates the above term, and the resulting vorticity in the exit plane is positive. However, for the uniform profile case, this latter component vanishes, and the vorticity in the exit plane is negative. This leads to the existence of regions of negative vorticity which move downstream and eventually break apart. It is still questionable to the author whether or not the specification of a vanishing radial velocity in the exit plane corresponds to a natural phenomenon which would not have to be forced in an experimental study. It is still unresolved whether the existence of embedded regions of negative vorticity is due to the dynamics of the flow field on the specified velocity distributions in the exit plane. Further numerical studies and experiment are needed to answer this question. Another difference in the flow fields is the existence of a local maximum in the radial profile of the axial velocity as shown in Figure 49 for the uniform profile case. For the case of the parabolic profile in which the gradients were not quite so high, the largest value of the axial velocity always occurred at the center line.

For both cases investigated in this research, the numerical solution gives a description of the flow field which is that of a vortex ring. After the cessation of flow from the exit plane, the behavior of each vortex indicates the slow decay of the angular velocities by the action of the viscous stresses. Since the magnitude of the axial velocity component near the center line is indicative of the velocity of the vortex ring, the slow decay of the velocities in the flow field is consistent with the sustained velocity which is observed in a smoke ring. 
CHAPTER V

\section{INVESTIGATION OF A SLOWLY DIFFUSING VORTEX RING}

In this chapter the results of the parabolic profile case are analyzed to obtain an understanding of the behavior of the fully developed vortex ring. The time period investigated is for $t>20$, the time after which the flow in the exit plane has ceased. The influence of the exit plane velocity on the moving ring is thus avoided. The geometry and velocity of the ring are computed as well as the circulation of the vortex. These parameters not only are of interest in themselves but also are necessary for a comparison of the numerical results with those which can be predicted by two existing models of vortex flow. The models are the vortex ring as presented by Lamb [7] and Hill's spherical vortex as given by Ringleb [9]. Only the results of the parabolic profile case are used because it is found that the results of the uniform profile case can add little to the insight already gained. Lamb's model does not appear to be a good approximation to the behavior of the vortices of this research. In fact, the parameters needed for its successful application must vary by several orders of magnitude from those which are calculated here. Since the needed variation does not exist in the results of the uniform profile, no correlation is attempted using its solution. On the other hand, the speed of the ring calculated from Hill's model does compare quite well with the speed computed numerically for the parabolic profile 
case. This correlation is also expected for the ring generated by the uniform velocity profile since here the dependent variables are of the same order of magnitude as those of the parabolic profile case.

\section{Computation of the Axial Location of the Vortex Center}

For simplicity the vortex is assumed to be traveling in the axial direction only; the radial movement is neglected. When the radial location of the vortex center is computed, this assumption can be verified. At any particular time, the vortex center lies somewhere on the locus of points for which the velocity is oriented in the axial direction. This is illustrated by the line of symmetry on the typical streamline pattern of Figure 52. As a consequence of neglecting the radial motion of the vortex, this line of symmetry is normal to the center line of the flow field.

Also shown in Figure 52 are two columns of grid points labeled $i$ and $i+1$ which lie upstream and downstream of the vortex center. The mesh spacing has been increased here for illustrative purposes. These axial stations may be found quite easily numerically. Over the vast majority of the mesh points labeled $i$ the radial velocity is negative, and over the vast majority of mesh points labeled $i+1$ the radial velocity is positive. At any radial station $j$, linear interpolation determines the axial co-ordinate between the mesh points $i$ and $i+1$ at which the radial velocity vanishes. The average axial coordinate over all $j$ is taken to be the axial location of the vortex center at any particular time. These points are shown as the triangles in Figure 53. 


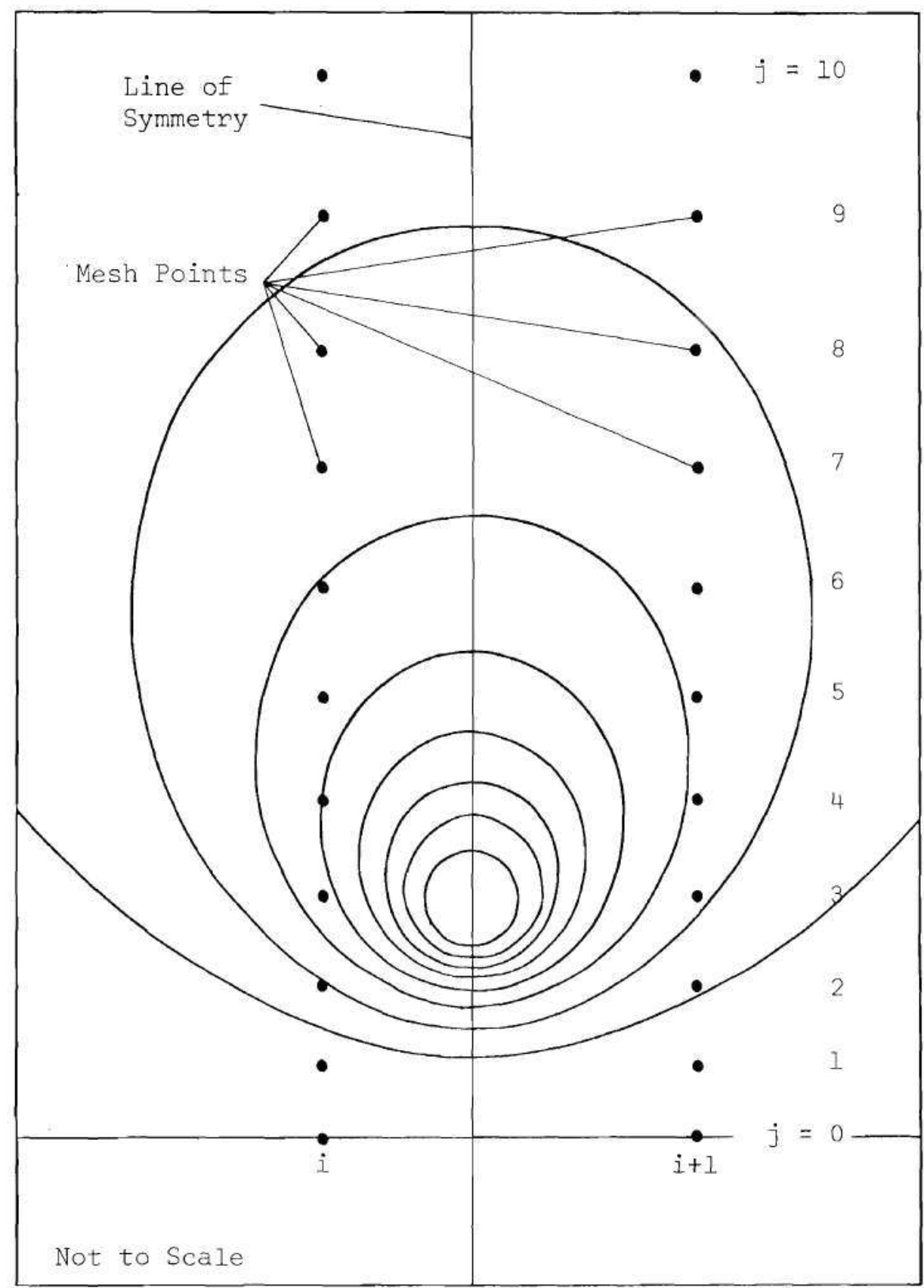

Figure 52. Illustration for Calculation of the Vortex Center 


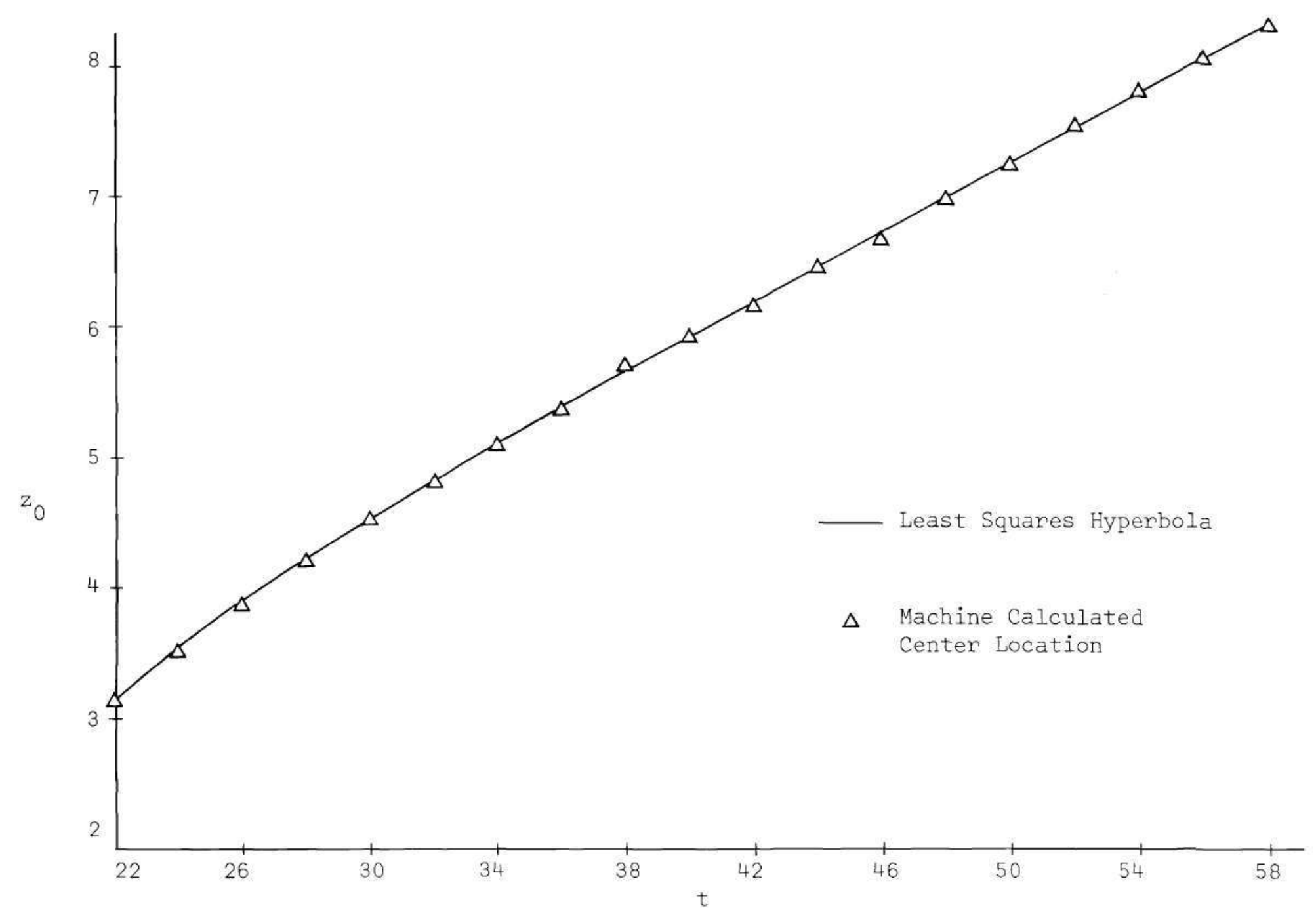

Figure 53. Axial Location of Vortex Center as Function of Time--Parabolic Profile 


\section{Computation of the Vortex Velocity}

Now that the axial location of the vortex is known as a function of time, the ring velocity may be calculated quite easily by the expression

$$
v_{z_{0}}=\frac{d z_{0}}{d t}
$$

where $v_{z_{0}}$ is the vortex velocity and $z_{0}$ is the axial location of its center. Unfortunately, there is enough deviation in the numerical values of $z_{0}$ to prohibit the use of the numerical approximation

$$
v_{z 0}(t)=\frac{z_{0}(t+\delta t)-z_{0}(t-\delta t)}{2 \delta t}
$$

When such a finite difference equation is employed, the resulting velocities do not fall on a curve which shows a decreasing ring velocity with increasing time.

A curve was computed using the center locations of Figure 53 by fitting a second degree polynomial by the method of least squares to the argument

$$
\left(z_{0}-w\right)^{2}
$$

Here $\mathrm{W}$ is a constant denoting the ordinate of the center of a hyperbola. The parameter $W$ was varied over a wide range until the average deviation between the numerically computed values of $\mathrm{z}_{0}$ and the curve was minimized. The result of this computation is shown by the curve 
of Figure 53. The maximum deviation between the hyperbola and the axial locations of the center is about 1 per cent, and the average deviation is about $3 / 10$ of a per cent.

The derivative of this hyperbola with respect to time, i.e., the ring velocity, is presented in Figure 54.

\section{Computation of the Radial Location of the Vortex Center}

Once the velocity of the ring is known at any given time, the fixed co-ordinate system may be transformed to one moving with the ring. In the moving co-ordinate system, the center of the vortex appears as a point of zero velocity. Since the dependent variables which are retained by the computer are the vorticity and the stream function, the co-ordinate transformation must be effected by manipulation of the stream function. In the $z, r$ plane, the axial velocity in the moving co-ordinate system at a particular time is given by

$$
v_{z m}(z, r)=v_{z}(z, r)-v_{z 0}
$$

where $v_{z m}$ is the axial velocity at the point $z, r$ in the moving coordinate system, $v_{z}$ is the axial velocity at the corresponding location in the fixed co-ordinate system, and $v_{z 0}$ is the ring velocity at this particular time. Rewriting Equation (74) in terms of the stream function results in the relation

$$
\frac{1}{r}\left(\frac{\partial \psi}{\partial r}\right)_{m}=\frac{1}{r}\left(\frac{\partial \psi}{\partial r}\right)_{f}-v_{z 0}
$$




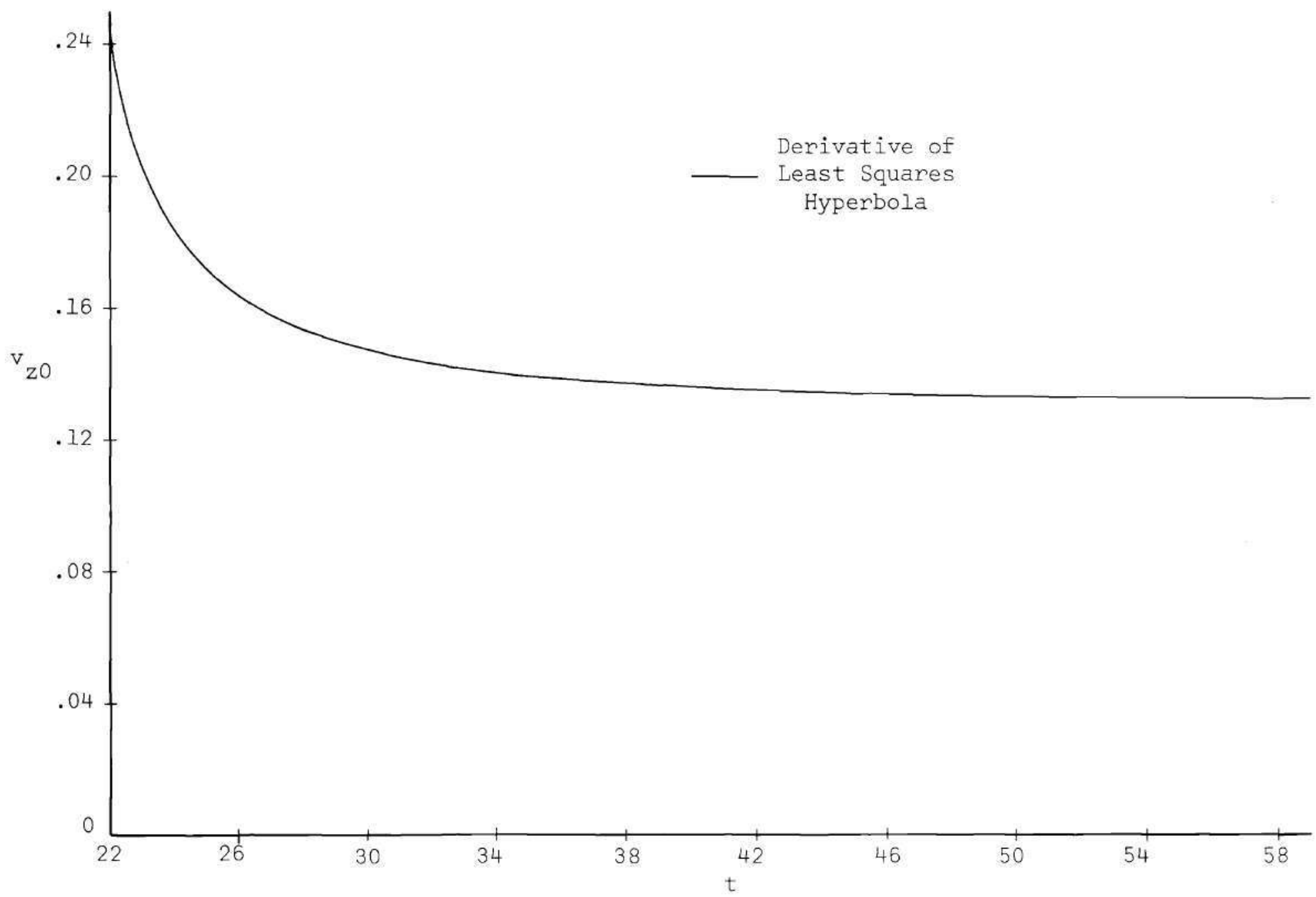

Figure 54. Propagation Velocity of Vortex as Function of Time--Parabolic Profile 
In the finite $\xi, n$ plane this becomes

$$
\frac{b(1-n)}{x}\left(\frac{\partial \psi}{\partial \eta}\right)_{m}=\frac{b(1-n)}{x}\left(\frac{\partial \psi}{\partial \eta}\right)_{f}-v_{z 0}
$$

Approximating this expression by central differences and multiplying the equation through by $x \neq 0$ gives, after simplifying,

$$
\begin{aligned}
\psi_{m}(i, j+1)=\psi_{m}(i, j-1) & +\left[\psi_{f}(i, j+1)-\psi_{f}(i, j-1)\right] \\
& -\frac{2 v_{z O} X}{b(M-j)}
\end{aligned}
$$

where

$$
x=r=\frac{1}{b} \ln \left(\frac{M}{M-j}\right) \text {. }
$$

However, on the center line $\psi=0$, and the multiplication of Equation (76) by $x$ to obtain Equation (77) cannot be performed. Thus, Equation (77) is not valid for $j=0$.

This difficulty may be resolved by noting that on the center line

$$
v_{z}(z, 0)=\operatorname{limit}_{r \rightarrow 0} \frac{1}{r} \frac{\partial \psi}{\partial r}=\left.\frac{\partial^{2} \psi}{\partial r^{2}}\right|_{r=0}
$$

This implies that in the transformed plane

$$
v_{z}(\xi, 0)=b^{2}\left(\frac{\partial^{2} \psi}{\partial \eta^{2}}\right)
$$


where the first derivative of the stream function vanishes due to symmetry. Employing Equation (78), Equation (74) may be rewritten on the center line as

$$
b^{2}\left(\frac{\partial^{2} \psi}{\partial n^{2}}\right)_{m}=b^{2}\left(\frac{\partial^{2} \psi}{\partial n^{2}}\right)_{f}-v_{z 0}
$$

Expressing this in central differences, applying the symmetry condition that

$$
\psi(i, 1)=\psi(i,-1)
$$

and simplifying yields

$$
\psi_{m}(i, I)=\psi_{m}(i, 0)+\psi_{f}(i, I)-\psi_{f}(i, 0)-\frac{v_{z 0}}{2 b^{2} M^{2}}
$$

Finally, since the stream function can be specified within an additive constant, the stream function at the center line is arbitrarily assigned a convenient value,

$$
\psi_{m}(i, 0)=0
$$

Equations (81), (80), and (77) allow the calculation of the stream function distribution on the center line, on the mesh points immediately next to the center line, and throughout the rest of the field, respectively, in a co-ordinate system which is moving with the speed 
of the vortex ring at the particular time of interest.

Once the stream function is known in the moving co-ordinate system, the corresponding velocity distribution may be computed. The center of the vortex corresponds to the location in the flow field which has zero velocity. This point is calculated at any time $t$ by finding the point at which $\mathrm{v}_{\mathrm{zm}}$ vanishes on the line which marks the axial location of the vortex center. Linear interpolation is used. The results given in Figure 55 show that the radial location of the vortex center is fairly constant. This behavior verifies the original assumption that the radial movement of the ring may be neglected.

Figure 55 illustrates that the radius of the vortex ring takes a representative value of about

$$
r_{0} \stackrel{\sim}{=} 1.18
$$

However, the vorticity distributions of Figures 21 through 29 show that this value is a little high. This in turn indicates that the velocity of the ring is a little low. This difference can probably be attributed to the linear interpolations which were performed in the finite $\xi, n$ plane.

\section{The Numerical Results in the Moving Co-ordinate System}

As a result of the calculation of the vortex center, the flow field is now known in a system moving with the speed of the vortex. Figure 56 is representative of the vorticity distribution and the streamline pattern in the moving co-ordinate system. This figure 


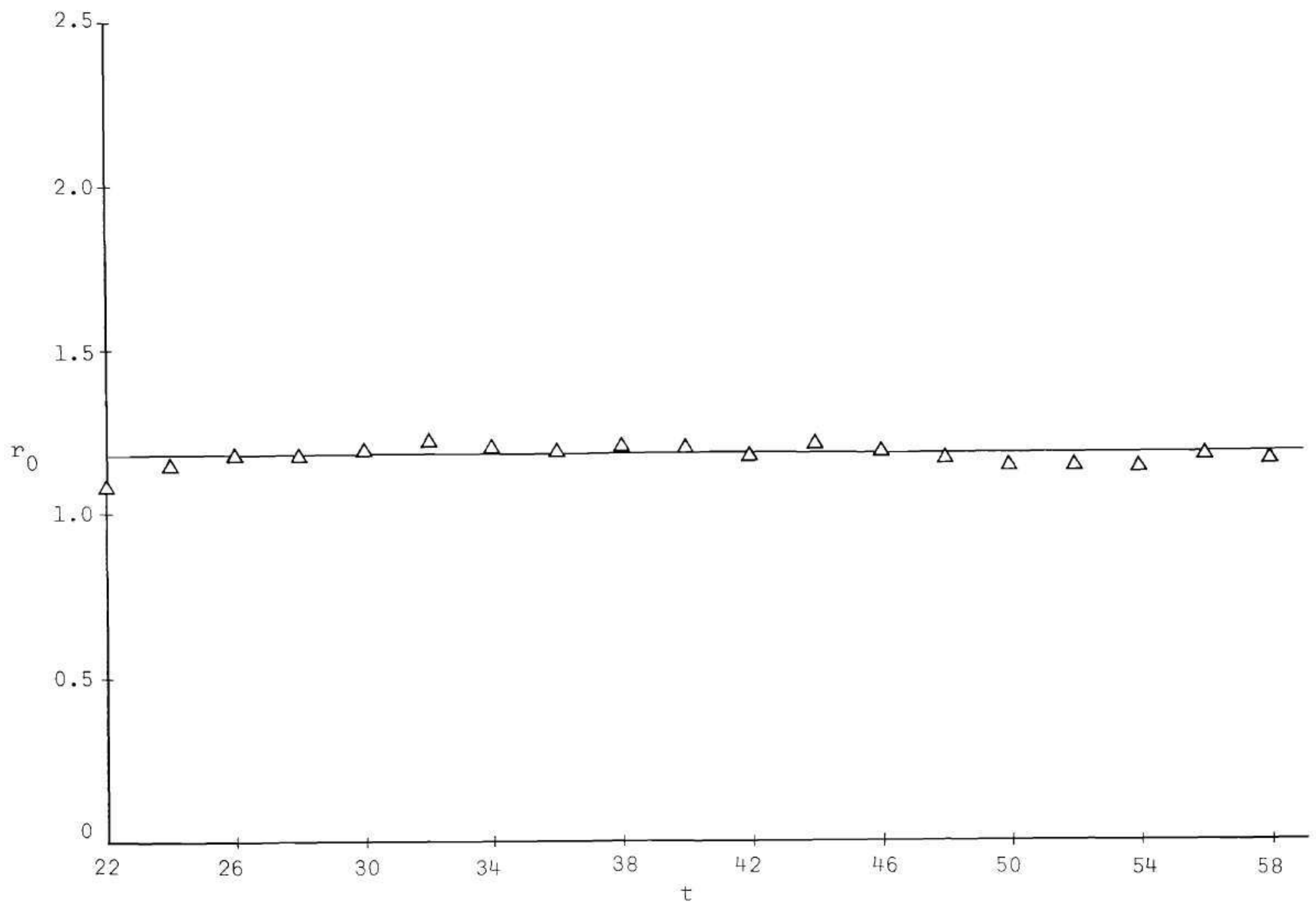

Figure 55. Radial Location of Vortex Center as Function of Time -- Parabolic Profile 


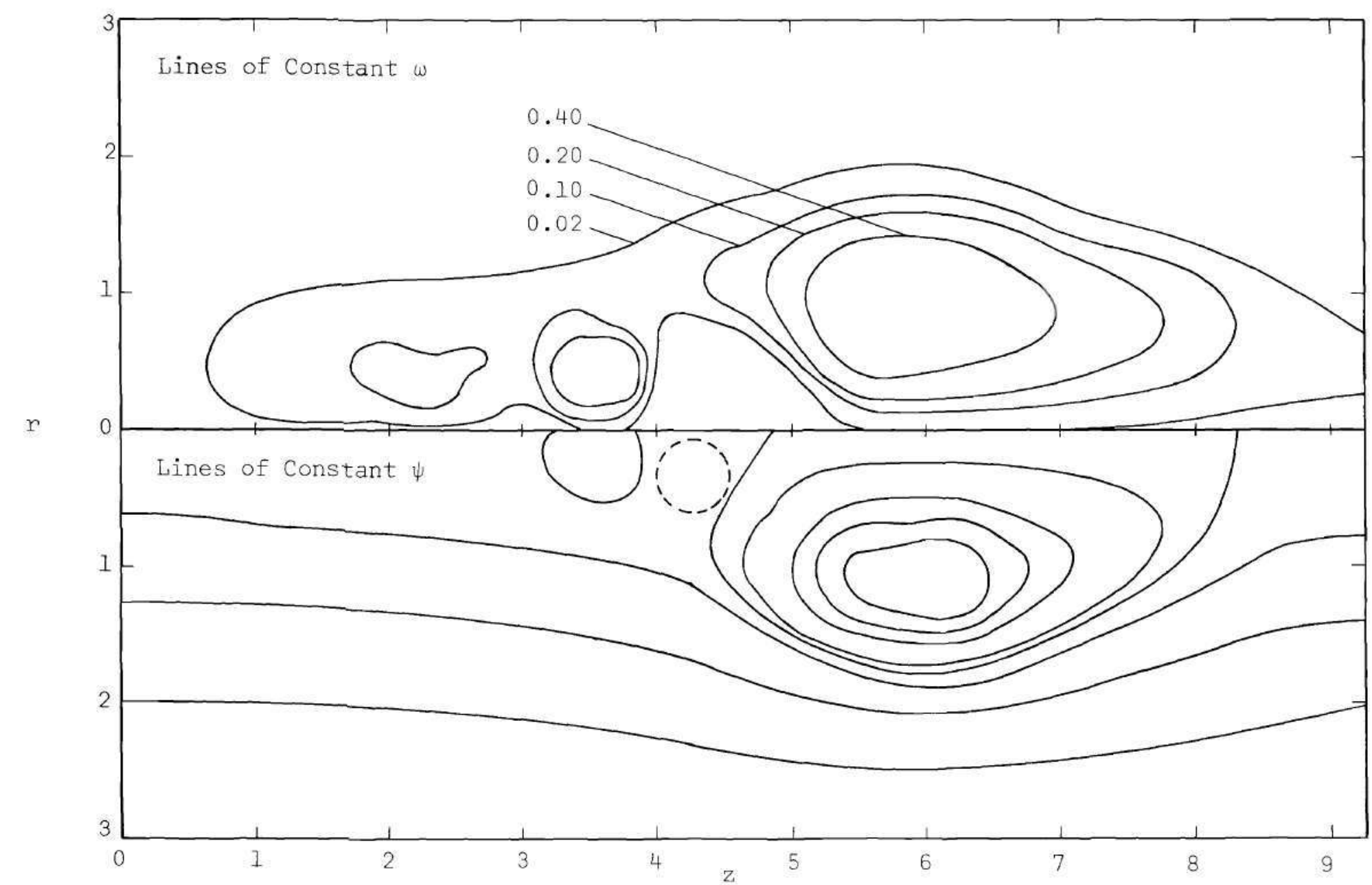

Figure 56. Vorticity Distribution and Streamline Pattern at $t=40$-- Parabolic Profile Moving Co-ordinate System 
corresponds to the results of Figure 25 in the fixed co-ordinate system. As mentioned previously, the vorticity distribution is unaffected when a uniform velocity is added to or subtracted from the velocity of the flow field. However, the streamline pattern does show a striking difference when it is compared to the pattern of the fixed co-ordinate system. In the moving co-ordinates the center of the streamline pattern does correspond with the center of the vortex. It can be seen that the center of the streamlines compares quite well with the maximum value of the vorticity.

In Figure 56 at $\mathrm{z} \cong 3.5$, a small vortex which rotates in the same direction as the vortex ring may be observed. Between this vortex and the vortex ring, there exists another secondary vortex which rotates in the opposite direction. This is indicated by the dashed streamline in the figure. The behavior of this secondary vortex which rotates in the opposite direction has already been well documented both in Figure 25 and the discussion accompanying that figure.

Finally, it may be noted that the streamlines of Figure 56 bear a striking resemblance to those of a spherical vortex. The latter streamlines are illustrated in Figure 57. The spherical vortex is the axisymmetric analog to a pair of standing vortices in a flow field of uniform velocity. A correlation between the spherical vortex and the vortex ring in a moving co-ordinate system will be made in a later section.

\section{Calculation of the Circulation}

For a velocity distribution in a co-ordinate system either fixed 


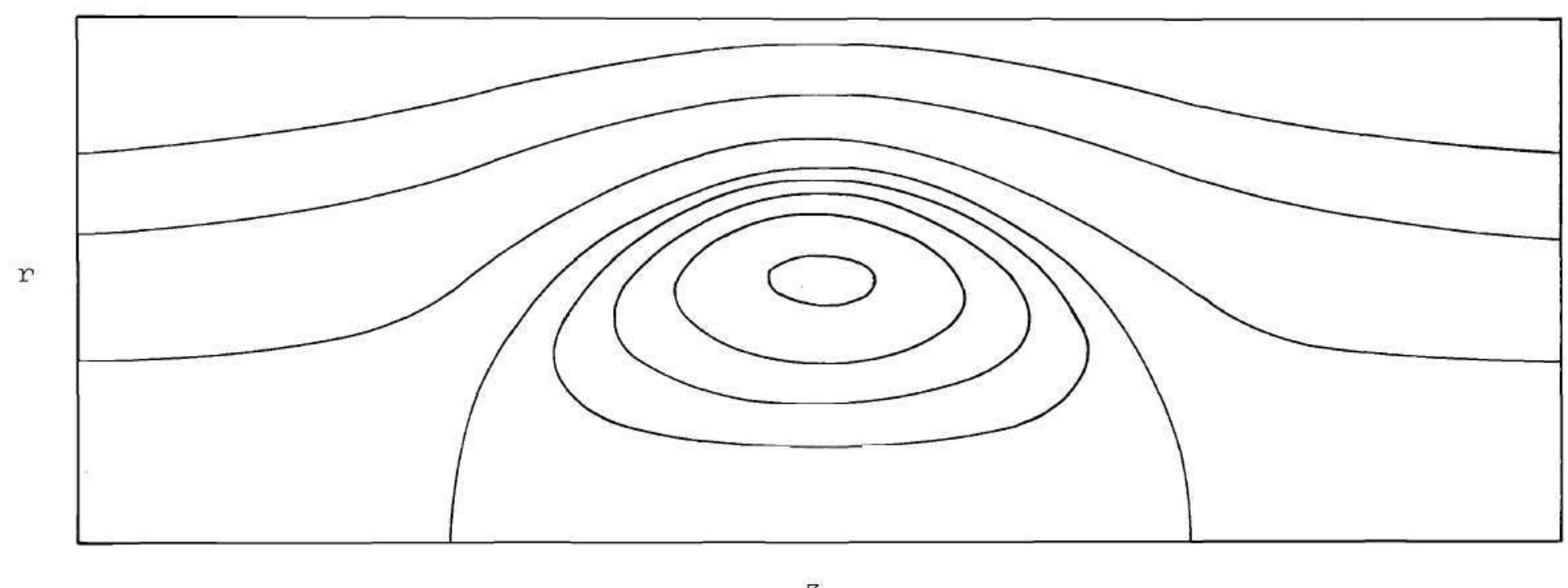

Figure 57. Streamline Pattern of Hill's Spherical Vortex as Given by Ringleb [9] 
in space or moving with the speed of the ring, the circulation may be computed by evaluation of the cyclic integral which is presented in vector form as

$$
\Gamma=\oint \bar{V} \cdot d \bar{s}
$$

where $\bar{V}$ is the velocity vector along the path of integration and d $\bar{s}$ is the incremental path length. This equation may be written in scalar form as

$$
\Gamma=\oint\left\{v_{z} d z+v_{r} d r\right\}
$$

The paths of integration used in this study are streamlines. Figure 58 is presented to clarify the discussion of the numerical calculation of circulation at a particular time and along a particular streamline. Consider the portion of the $\mathrm{z}, \mathrm{r}$ plane and the accompanying mesh points illustrated in the figure. The location of the streamline corresponding to a given stream function can be found by linear interpolation between the values of stream function at adjacent grid points. These are also shown in Figure 58. If the line integrals of the form

$$
\Delta \Gamma=\int\left\{v_{z} d z+v_{r} d r\right\}
$$

are summed over discrete portions of the closed streamline, the resulting summation along the entire path is simply the circulation 


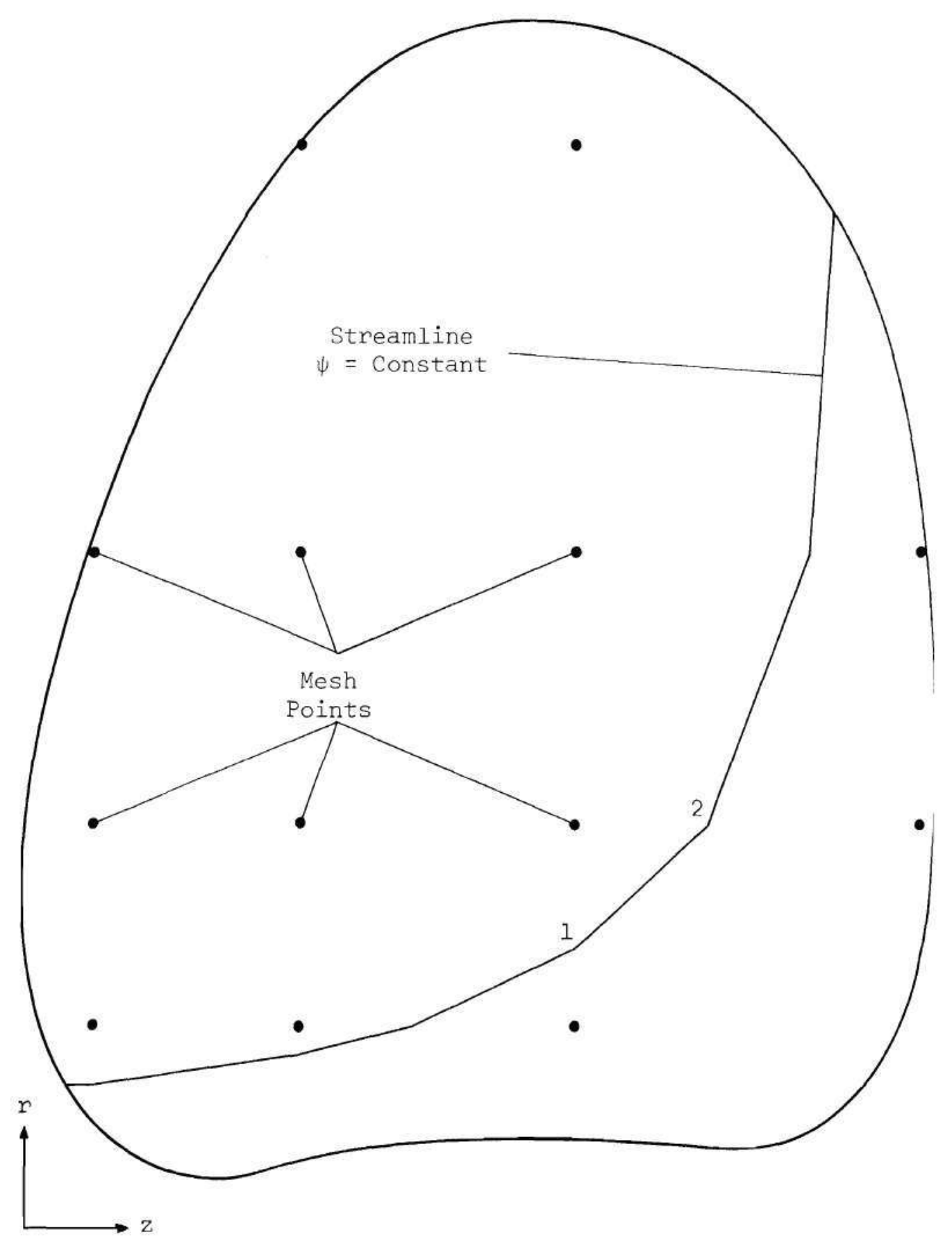

Figure 58. Portion of $\mathrm{z}, \mathrm{r}$ Plane Used in the Calculation of the Circulation 
of Equation (82). The problem of calculating the circulation is reduced to the computation of the line integral indicated by Equation (83) over a portion of the streamline. An incremental path length is shown by the points 1 and 2 in Figure 58. Under the assumption that the velocity components vary linearly over the line increment from point 1 to point 2, Equation (83) may be written

$$
\begin{aligned}
\Delta \Gamma & =\frac{1}{2}\left(z_{2}-z_{1}\right)\left[v_{z}\left(z_{1}, r_{1}\right)+v_{z}\left(z_{2}, r_{2}\right)\right] \\
& +\frac{1}{2}\left(r_{2}-r_{1}\right)\left[v_{r}\left(z_{1}, r_{1}\right)+v_{r}\left(z_{2}, r_{2}\right)\right]
\end{aligned}
$$

where the subscript refers to the point at which a particular parameter is being evaluated.

Figures 59 and 60 present the dimensionless circulation distributions in the fixed and moving co-ordinate systems respectively at various times as functions of the area enclosed by the paths of integration (streamlines). The dimensionless area is a product of a dimensionless radial and axial distance. Since some of the streamlines form closed paths only at very large distances from the center line, the only streamlines employed in the computation of the circulation are the ones which form closed paths within the large finite region bounded by the grid lines $i=0, j=0, i=M-1$, and $j=M-1$. The circulation is also calculated along the closed path composed of these grid lines. This value is taken as an approximation to the circulation of the quarter-infinite flow field. 


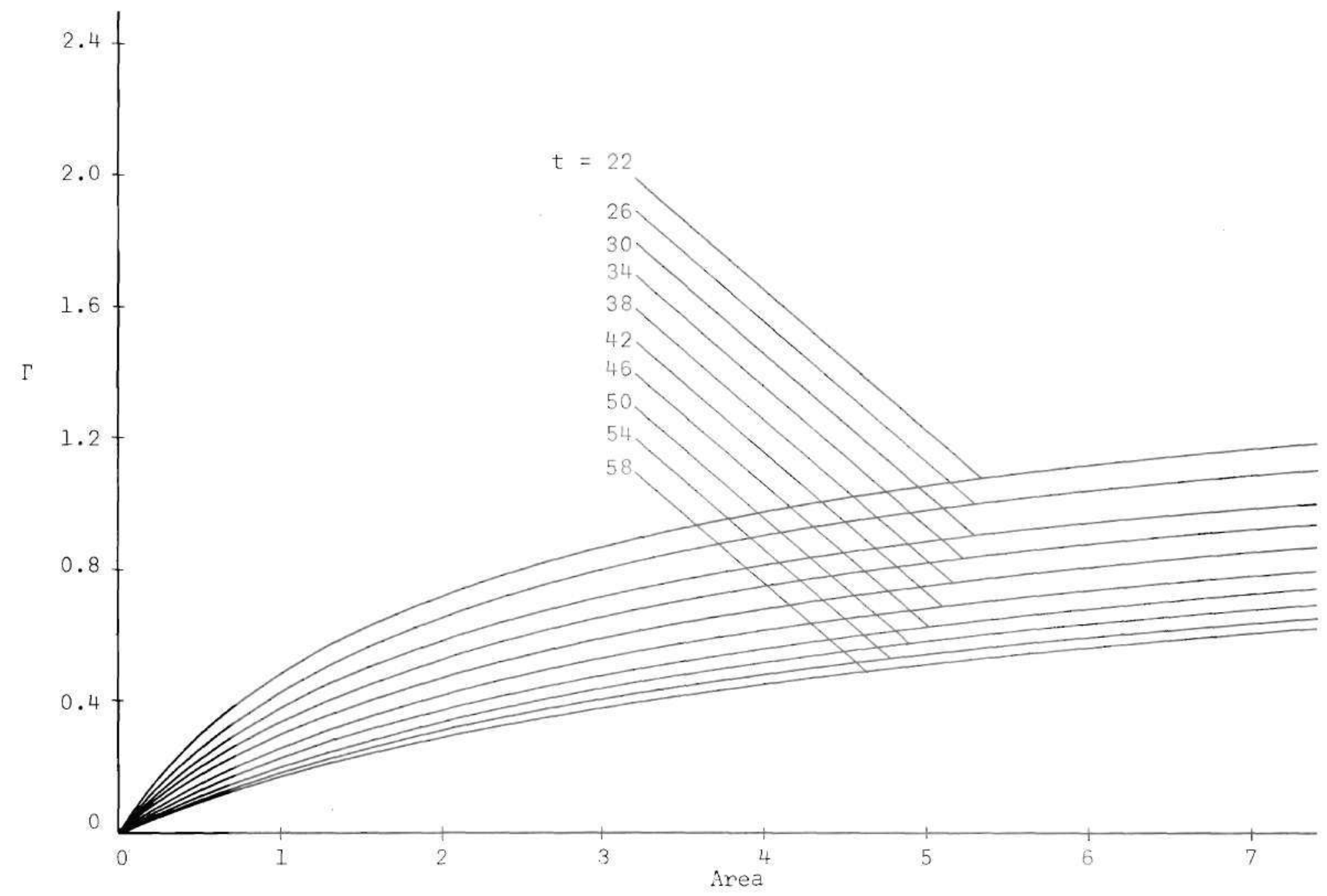

Figure 59. Circulation Along Streamline as Function of Area Enclosed by Streamline, Parabolic Profile--Fixed Co-ordinate System 


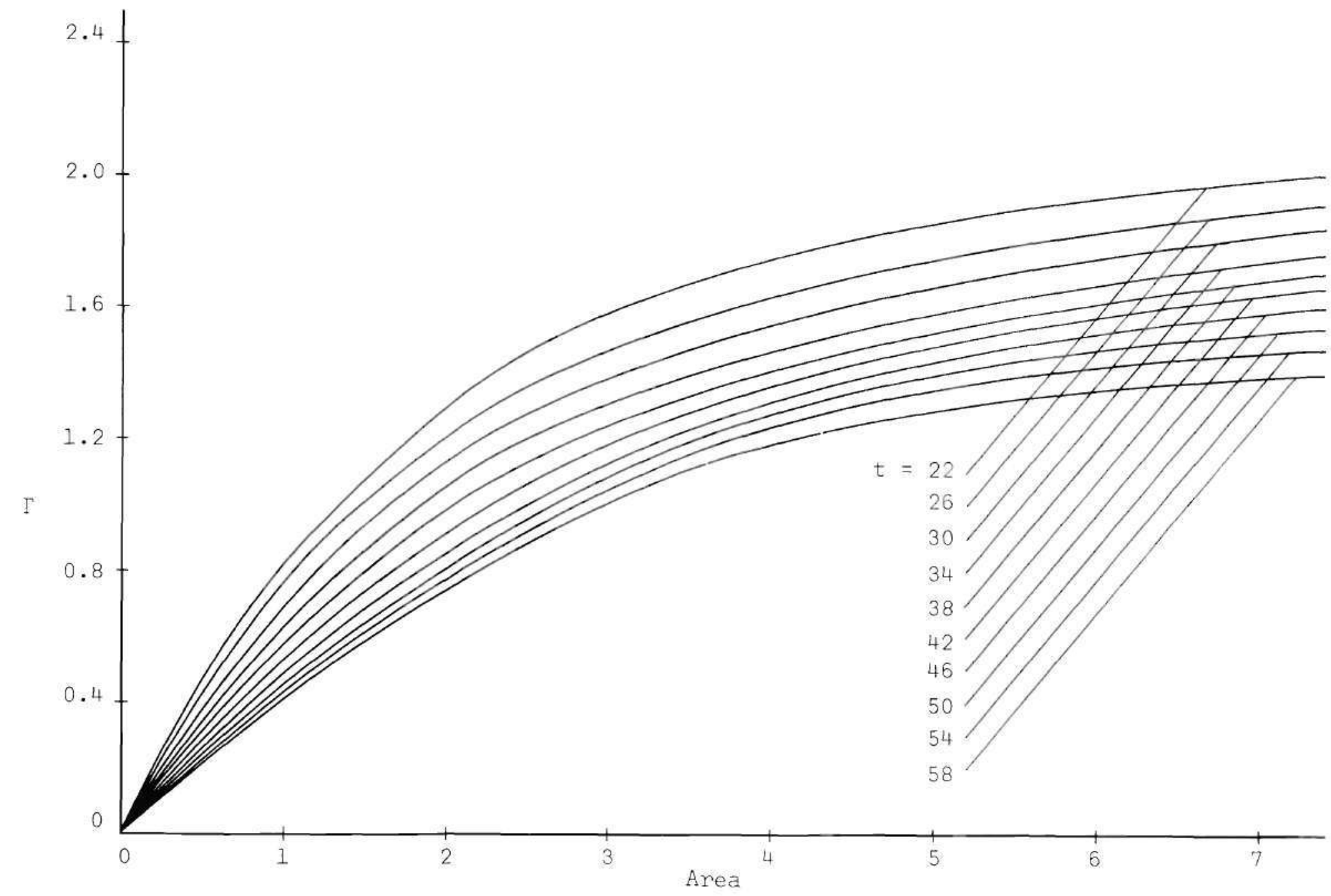

Figure 60. Circulation Along Streamline as Function of Area Enclosed by Streamline, Parabolic Profile--Moving Co-ordinate System 
Consider now the circulation distributions in the fixed coordinate system as illustrated in Figure 59. Since the center of the streamline pattern in the fixed co-ordinate system does not coincide with the center of the vortex, some of the streamlines which surround smaller regions of the flow field do not enclose the center of the vortex. At any particular time the circulation vanishes at the center of the streamline pattern where the area is zero. The circulation then increases asymptotically to the value calculated over the large rectangular region enclosed by the grid lines $i=0, j=0, i=M-1$, and $j=M-1$. For Figure 59, this corresponds to an area of about 50. The circulation along this line is about twice that of the circulation along the largest closed streamline. Since the circulation may also be calculated by the expression

$$
\Gamma=\iint \omega \mathrm{dA}
$$

where the double integral denotes integration over a region, it can be seen that the decreasing slope of a particular circulation curve for larger and larger enclosed areas indicates a decreasing vorticity. This behavior has already been observed on the plots of the vorticity distribution. Although the center of the streamline pattern in the fixed co-ordinate system cannot be ascertained exactly, at any particular time the slope of the circulation curve for a vanishing enclosed area compares well with the vorticity in the region near the center of the streamline pattern. 
Eigure 59 also indicates that the circulation decreases with time. This is attributed to the shear stresses experienced by the flow field. The action of viscosity steadily diminishes the velocity gradients, and in so doing causes the circulation to steadily decrease with time.

In Figure 60 the circulation is presented in the moving coordinate system. Each curve represents the circulation as a function of area in a co-ordinate system moving with the speed of the ring at the time indicated on the curve. Here, the center of the streamines corresponds closely to the center of the vortex. Hence, for small areas and at a particular time, the slope of the curve in the moving co-ordinate system is greater than the corresponding slope in the fixed system. This is due to the fact that the average vorticity over a given area in the moving system is greater than that for an equal value of the area in the fixed system. The closed streamlines of the moving system all enclose the vortex center where the vorticity is a maximum. As the area surrounded by the streamlines of integration increases, the difference between the circulations of the fixed and moving systems decreases until the two circulations are equal for the large but finite rectangular region whose boundaries correspond to $i=0, j=0, i=M-1$, and $j=M-1$. This equality is expected since the circulation along a given path does not change if a uniform velocity is added to the field.

Aside from the differences already mentioned, the behavior of the curves of Figure 60 is similar to that of Figure 59. The circulation is zero at the origin, and it increases to the circulation 
computed along the rectangle. The circulation along the largest enclosed streamline remains about 83 per cent of the circulation computed along the rectangle. Hence, in the moving co-ordinate system, the closed streamlines around the vortex center contain by far the larger portion of the circulation of the field. As time progresses the action of viscosity causes the decay of the velocity gradients, and the circulation decreases with time accordingly.

\section{Correlation of Existing Vortex Models}

Now that the geometry, circulation, and velocity of the vortex ring have been calculated, a comparison of some of the numerical results may be made with those of existing vortex models. As mentioned at the beginning of this chapter, the models are the Lamb vortex ring and Hill's spherical vortex. The name of the first model is consistent with the current literature because many authors state that the result may be found in Lamb although Lamb himself points out that the expression for the velocity of the vortex ring was first given without proof by $\operatorname{Sir} W$. Thompson in an appendix to a translation of a paper by Helmholtz. Lamb's Vortex Ring

The analysis of Lamb is outlined cursorily in this paragraph although a detailed derivation may be found in Appendix J. A vortex ring may be considered as a system of circular vortex filaments. It is assumed that the vorticities of all the elements are equal; therefore, the core rotates as a solid body. Outside the core the motion of the fluid is irrotational. An energy equation is derived which 
relates the energy of the system of filaments to the energy of the core. The velocity of the ring appears in a term of this expression, and the whole equation connects the velocity of the ring to the kinetic energy, the vorticity or strength, and the configuration of the ring. The kinetic energy may be expressed as a volume integral of the product of the stream function and the vorticity within the core, and the stream function in turn is given by Lamb as the integral of a complicated expression of elliptic integrals. In order to evaluate this integral, a simplification must be made. It is assumed that the core of the ring is circular primarily for convenience, but more important is the assumption that the radius of the core is much smaller than the radius of the ring. This allows a simplification of the elliptic integrals and their subsequent integration. Computation of the stream function and thus the kinetic energy of the ring gives finally the expression of a simple equation for the ring velocity, namely

$$
v_{z_{0}}=\frac{\Gamma}{4 \pi r_{0}}\left\{\ln \left(\frac{8 r_{0}}{r_{L}}\right)-\frac{1}{4}\right\}
$$

where

$$
\begin{aligned}
& v_{z 0}=\frac{v_{z 0}^{*}}{U} \text {, the dimensionless velocity of the ring } \\
& r_{0}=\frac{r_{0}^{*}}{R} \text {, the dimensionless radius of the ring } \\
& r_{L}=\frac{r_{L}^{*}}{R} \text {, the dimensionless radius of the core }
\end{aligned}
$$




$$
\Gamma=\frac{\Gamma * \mathrm{U}}{\mathrm{R}} \text {, the dimensionless circulation of the ring }
$$

The starred quantities denote dimensional variables. Remember that Lamb has assumed that $r_{L} \ll r_{0}$.

The results of the numerical solution shown in the previous chapter now allow the evaluation of all the parameters of the Lamb vortex model except the core radius $r_{L}$. This may be determined from Equation (85). This is one of the purposes of this research: first, to ascertain whether or not the Lamb vortex model corresponds to the results of this study, and second, to calculate representative values of the core radius which may be used in other analyses. Obviously, the procedure used to calculate the core size and verify Lamb's vortex model must be applied only at specific times in order to be consistent with Lamb's analysis which is independent of time. The circulation and vorticity of the core of the vortex are constant because no mechanism exists for their decay.

The computational method for evaluating the parameters of Equation (85) from the results of the numerical study of the parabolic profile case will now be discussed. Since the velocity and radius of the ring are already presented in Figures 54 and 55 as functions of time, the only parameter left to calculate is the circulation of the vortex ring.

The circulation may be computed by the equation

$$
\Gamma=\iint\left(\frac{\partial v_{r}}{\partial z}-\frac{\partial v_{z}}{\partial r}\right) d r d z
$$


where the double integral denotes the integration of the vorticity over a portion of the $z, r$ plane. For the solid body rotation of the assumed vortex core,

$$
\frac{\partial v_{r}}{\partial z}-\frac{\partial v_{z}}{\partial r}=\bar{\omega}=\text { constant }
$$

where the constant is just twice the angular velocity of the core. Hence, within the core the circulation is given by

$$
\Gamma=\bar{\omega} \iint \mathrm{dr} d \mathrm{z}=\bar{\omega} \pi \mathrm{s}^{2} \text { for } \mathrm{s} \leq \mathrm{r}_{L}
$$

where the integration is performed over a circular region concentric with the core. The circulation within the core may therefore be expressed as a linear function of the area of integration. Hence,

$$
\Gamma=\vec{\omega} A
$$

Outside of the core, the vorticity of the fluid is zero, and the circulation is constant for any region of integration which includes the core. These considerations lead to the depiction of the circulation about the vortex center as that of the two linear segments shown in Figure 61 .

On the other hand, the circulation distribution for the viscous vortex follows the curve shown in Figure 61. This behavion is consistent with the earlier plots of the circulation as a function of the 


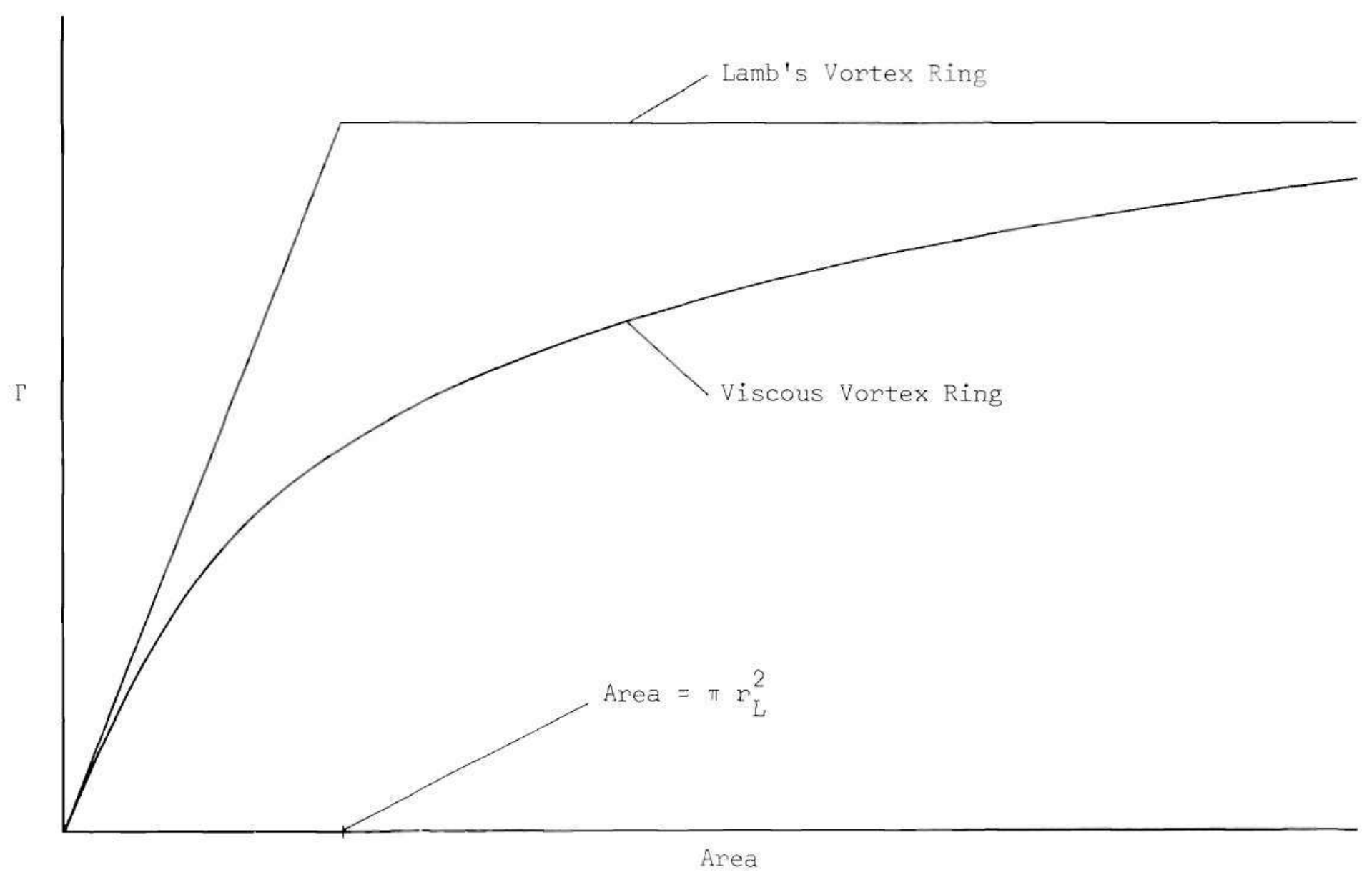

Figure 61. Postulated Comparison of the Circulations of Lamb's Vortex Ring and the Viscous Vortex Ring 
area enclosed by the integration. The circulation is zero at the center of the vortex where the area of integration is zero, and it increases as the area increases until it reaches a maximum which corresponds to the circulation of the entire flow field.

A number of attempts were made to use the results of the numerical solution to find the core radius. In the first of these, the circulation within the core is expressed as

$$
\Gamma=\bar{\omega} A
$$

where $\bar{\omega}$ is the numerically calculated vorticity at the vortex center. Remembering that at the edge of the core

$$
A=\pi r_{L}^{2}
$$

Equation (85) may be rearranged to give the implicit expression

$$
\left(\frac{r_{0}}{r_{L}}\right)^{2}=\frac{r_{0} \bar{\omega}}{4 v_{z_{0}}}\left(\ln \left(8 \frac{r_{0}}{r_{L}}\right)-\frac{1}{4}\right)
$$

Since $r_{0}, \bar{w}$, and $v_{z 0}$ are all known parameters at a particular time, the value of $\left(r_{0} / r_{L}\right)$ may be calculated quite easily by iteration. Any initial guess at the unknown parameter may be substituted in the right-hand side of Equation (88), and an improved guess is calculated on the left-hand side. This value is in turn substituted into Equation (88), and the iteration continues until successive iterates of the parameter 
$\left(r_{0} / r_{L}\right)$ agree within some degree of approximation. This procedure was employed at a number of discrete times, and some sample results are given in Table 5.

Table 5. Core Size of Lamb's Vortex Ring Using Vorticity

\begin{tabular}{lcccc}
\hline$t$ & $r_{0}$ & $v_{z 0}$ & $\bar{\omega}$ & $r_{\mathrm{L}} / r_{0}$ \\
\hline 32 & 1.18 & 0.144 & 0.9 & 0.46 \\
40 & 1.18 & 0.137 & 0.7 & 0.52 \\
48 & 1.18 & 0.134 & 0.5 & 0.63 \\
56 & 1.18 & 0.132 & 0.5 & 0.63 \\
\hline
\end{tabular}

From these calculations it can be seen that the radius of the core is not small compared to the radius of the ring although this assumption was made in the analysis of the solid core model.

In a second attempt at the calculation of the core radius, the circulation of the ring was assumed to be that calculated for the large finite region bounded by the grid lines $i=0, j=0, i=M-1$, and $j=M-1$. This value of the circulation is taken to be an approximation of the circulation of the quarter-infinite region. Some sample results are given in Table 6 . 
Table 6. Core Size of Lamb's Vortex Ring Using Circulation

\begin{tabular}{lllll}
\hline $\mathrm{t}$ & $\mathrm{r}_{0}$ & $\mathrm{v}_{\mathrm{ZO}}$ & $\mathrm{T}$ & $\mathrm{r}_{\mathrm{L}} / \mathrm{r}_{0}$ \\
\hline 32 & 1.18 & 0.144 & 1.97 & 2.21 \\
40 & 1.18 & 0.137 & 1.79 & 2.00 \\
48 & 1.18 & 0.134 & 1.74 & 1.99 \\
56 & 1.18 & 0.132 & 1.67 & 1.94 \\
\hline
\end{tabular}

The core radius calculated from Equation (85) for these values of the circulation is greater than the radius of the ring. Clearly, this result is unrealistic.

Investigation of Equation (85) reveals that an increase in the circulation of the ring causes an increase in the core size for a given ring velocity. In fact, to obtain the vortex ring velocity indicated by the numerical solution of the viscid equations, a core radius which is small compared to the ring radius necessitates a small ring circulation in Equation ( 85 ). This circulation does not correspond to any circulation near the vortex center which is experienced by the viscous vortex of this analysis.

It is concluded that the model of a vortex ring proposed by Lamb has no correlation to the behavior of the viscous vortex ring of this study, nor does it accurately predict the ring velocity. Study of the various vorticity distributions presented reveals that the vorticity generated in the uniform profile case is higher than that of the parabolic case. Although the ring velocity appears to be somewhat 
higher in this case, the comparison with the Lamb model is expected to be no better. The conclusion for the uniform profile case is the same. For a given ring velocity, a core radius which is small compared to the ring radius dictates a ring circulation which is smaller than any experienced by the fluid at the vortex center. Hills' Spherical Vortex

The resemblance between the streamline pattern of the vortex ring in a moving co-ordinate system and the streamline pattern of Hill's spherical vortex has already been noted. In this section, a correlation is made between the results of the numerical model and the results of Hill's analysis as given by Ringleb [9]. The derivation is outlined in the next paragraph and given in detail in Appendix $k$. Physically a spherical vortex is the three-dimensional, axisymmetric analog to a standing vortex in a uniform flow field with the sphere being the separating streamline between the internal and external flows. Inside this streamline a vortex pattern appears, and outside the fluid behaves as it does in uniform irrotational flow over a sphere. Mathematically, the model arises by assuming that in a certain region of the flow the vorticity is proportional to the radial distance from the center line. The solution of the definition of vorticity equation within the sphere gives the streamline pattern of a confined vortex in terms of the unknown constant of proportionality between the vorticity and the spatial co-ordinate $r$.

The velocity from the known streamline distribution for irrotational flow around a sphere is matched on the sphere to the velocity of the vortex flow, and the resulting expression relates the vorticity 
distribution inside the sphere to the uniform velocity of the external flow at a great distance from the sphere. Integration of the vorticity over half of the cross section of the sphere yields a relation between the radius of the sphere, the velocity of the uniform field, and the circulation of a semi-circular area of the cross section. This equation is

$$
\mathrm{v}_{\mathrm{zO}}=\frac{1}{5} \frac{\Gamma}{\mathrm{a}_{0}}
$$

where

$$
\begin{aligned}
& \mathrm{v}_{\mathrm{zO}} \sim \text { dimensionless uniform velocity of the external flow } \\
& \mathrm{a}_{0} \sim \text { dimensionless radius of the sphere } \\
& \Gamma \sim \text { dimensionless circulation of } 1 / 2 \text { of the cross section } \\
& \quad \text { of the sphere }
\end{aligned}
$$

Obviously, Equation (89) must be applied to the results of the numerical model only at discrete times because there exists no mechanism for the decay of the circulation in the spherical model. However, observation of Figure 56 gives a more optimistic view to this correlation than the one attempted using the Lamb model. In the region of the sphere, the vorticity appears to be a strong function of the radial co-ordinate $r$ but only a weak function of the axial co-ordinate $z$. In addition, the analysis of the circulation of the vortex in the moving co-ordinate system reveals that the circulation within the closed streamline which intercepts the center line of symmetry is almost 85 per cent of the total circulation of the field. Hence, the assumption 
that the flow outside the sphere is irrotational is probably reasonable. The velocity of the uniform velocity field, or conversely the velocity of the vortex moving through a motionless fluid, is calculated in the following manner. At a particular time the circulation is calculated numerically from Equation (84) along the streamline which intercepts the center line of symmetry and thus approximately forms a semicircle. The radius of a semicircle having the same area as that enclosed by the streamline of interest is also computed. Substitution of the circulation and the radius of the sphere into Equation (89) yields the values of the uniform velocity $v_{z 0}$ at various times which are shown by the triangles of Figure 62 . The solid line of the figure is the numerically computed vortex ring velocity. The velocity of the Hill spherical vortex compares well to that of the vortex ring of this investigation.

\section{Concluding Remarks}

In this chapter, the vortex ring generated by a fluid possessing a parabolic velocity profile in the exit plane has been investigated. The velocity of the ring is found to decrease until it approaches a steady state value whereas the radius of the ring remains constant. The circulation is calculated along the streamlines of two co-ordinate systems at discrete times. The first system is fixed in space, and the second moves with the velocity of the ring. In both cases the circulation is zero at the center of the streamline pattern, and it increases asymptotically to the value of the circulation of the whole field when it is plotted as a function of the area enclosed by the streamlines. In the fixed co-ordinate system the circulation varies throughout the 


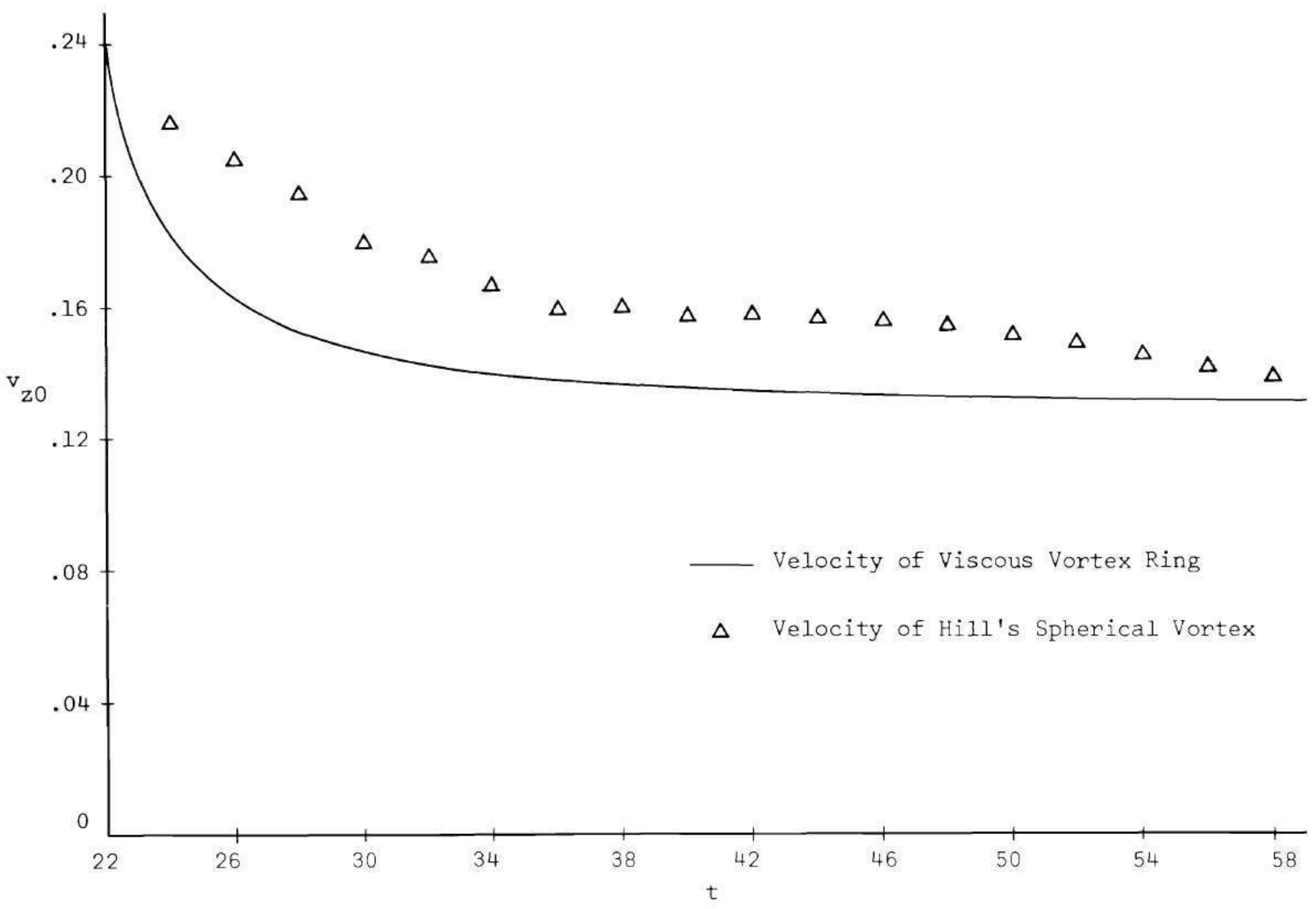

Figure 62. Propagation Velocity of Vortex as Function of Time 
field, but for the moving co-ordinate system the greatest amount of circulation is found in a region near the center of the vortex. The vortex ring model developed by Lamb which assumes the existence of a very small region of concentrated vorticity does not predict accurately the vortex velocity. This is due to the fact that the radius of the vortex core cannot be found for values of the circulation which actually occur near the vortex center. On the other hand, the spherical vortex model of Hill which assumes the existence of a rather large region of concentrated vorticity does predict quite accurately the vortex velocity. However, since both models approximate steady state phenomena, neither can predict the time rate of change of the circulation. Only the Navier-Stokes equations or the vorticity transport equation can describe the behavior of an unsteady viscous vortex ring. 


\section{CHAPTER VI}

\section{CONCLUSIONS AND RECOMMENDATIONS}

The generation and development of a viscous vortex ring has been investigated analytically for the unsteady, incompressible, threedimensional, axysymmetric flow of air in a large volume. The governing equations (the Navier-Stokes equations and the continuity equation) have been reduced to a system of two simultaneous partial differential equations (the vorticity transport equation and the definition of vorticity equation), and an implicit finite difference technique has been developed for their solution. Two specific problems have been investigated in detail, and the resulting vorticity, stream function, and velocity distributions have been presented. Finally, the numerical results of one of these problems have been studied further to obtain the geometry, speed, and circulation of the vortex ring. These parameters are used in a comparison of the behavior of the viscous vortex ring to the behavior of each of two existing vortex models.

\section{Conclusions}

The results of the investigation may be summarized in the following conclusions.

1. A technique has been developed which permits the first detailed analytical study of the generation and development of a viscous vortex ring. The quarter-infinite region of the mathematical model has been reduced by a simple transformation to a finite region over which 
the method of finite differences is practicable. The finite difference method of the investigation has been demonstrated to provide a stable and accurate solution to the governing equations.

2. The numerical results compare well with the limited experimental data available on the behavior of vortex rings. The streaklines which were calculated from the numerical results at the initial stages of the vortex development agree qualitatively with smoke pictures which have been obtained experimentally. However, in contrast to the findings of Akmetov and Kisarov [37] (this work became available during the preparation of the manuscript), the results of the present investigation indicate that the region of high vorticity near the vortex center extends over a fairly lange volume of the flow field.

3. The vortex ring proposed by Lamb which consists of a small, circular rotational core embedded in an irrotational field is not an accurate model for the viscous vortex ring of this research. On the other hand, the comparison of the propagation velocities with Hill's spherical vortex is good, and at any given time, the ring velocity compares within about 15 per cent with that predicted by the steady state spherical vortex. Although the details of the two flow fields do not compare well, perhaps it can be concluded that only the integrated effects are important, at least for the case studied here.

\section{$\underline{\text { Recommendations }}$}

\section{The Numerical Method}

A number of general comments are offered at this time concerning the application of the finite difference method to problems which require 
a solution over a region of space (e.g., the Navier-Stokes equations).

1. Further verification of the method proposed by Esch [22] for establishing boundary conditions should be pursued especially with respect to comparison of analytical results and experimental data. The Esch method was not used in this investigation. Perhaps this formulation of the boundary conditions may be extended to give a better statement of the Neumann conditions utilized on boundaries located at large values of the spatial co-ordinates.

2. The exponential spatial transformation appears to be advantageous to certain types of problems of external aerodynamics. The use of the transformation in these problems should be pursued. Although the transformed equations are somewhat more difficult to analyze for stability, the transformation itself is a neat and concise method for obtaining a large number of mesh points near the boundary and phenomenon of interest. In addition, a similar exponential transformation of time to a finite interval may prove useful in the computation of unsteady problems to obtain steady state results.

3. An investigation should be made to improve the speed of convergence of iterative techniques employed in solving highly nonlinear systems. The Problem of the Viscous Vortex Ring

In this section, a number of general suggestions are offered which pertain to the numerical solution of the generation and development of a viscous vortex ring.

1. It is suggested that in any further studies a Dirichlet condition, $\psi=0$, be applied for large $z$ and large $r$ after the flow 
has ceased in the exit plane. The Neumann condition was used throughout this study. The effect of this Dirichlet condition should be carefully noted and compared to the results given in this investigation.

2. The numerical method for the two problems--the uniform profile case and the parabolic profile case--should be investigated for a number of finer spatial meshes and smaller time increments. In this way the question of convergence can be answered with more positive assurance. In addition, the "stretching factors" used in the transformation should be changed to investigate the effect of varying the distance between the co-ordinate axes and the furthest finite grid point.

3. In order to better understand the mechanism by which the vortex ring is formed, the following recommendations are made:

a. The pressure should be calculated throughout the flow field at various times for the parabolic and uniform profile cases of this study. These distributions coupled with the vorticity and velocity distributions may help to explain the mechanism of vortex formation.

b. Similar problems should be calculated for very large Reynolds numbers. Perhaps the importance of the viscous stresses can be determined in this way. 4. The specification of a vanishing radial velocity in the exit plane is physically questionable. This question can be answered analytically by solving numerically the governing equations for the proposed physical model of Figure 63. Here, the trajectory of a piston in a tube to the left of the exit plane must be specified. Thus only the axial velocity component need be specified on the piston face. Because 


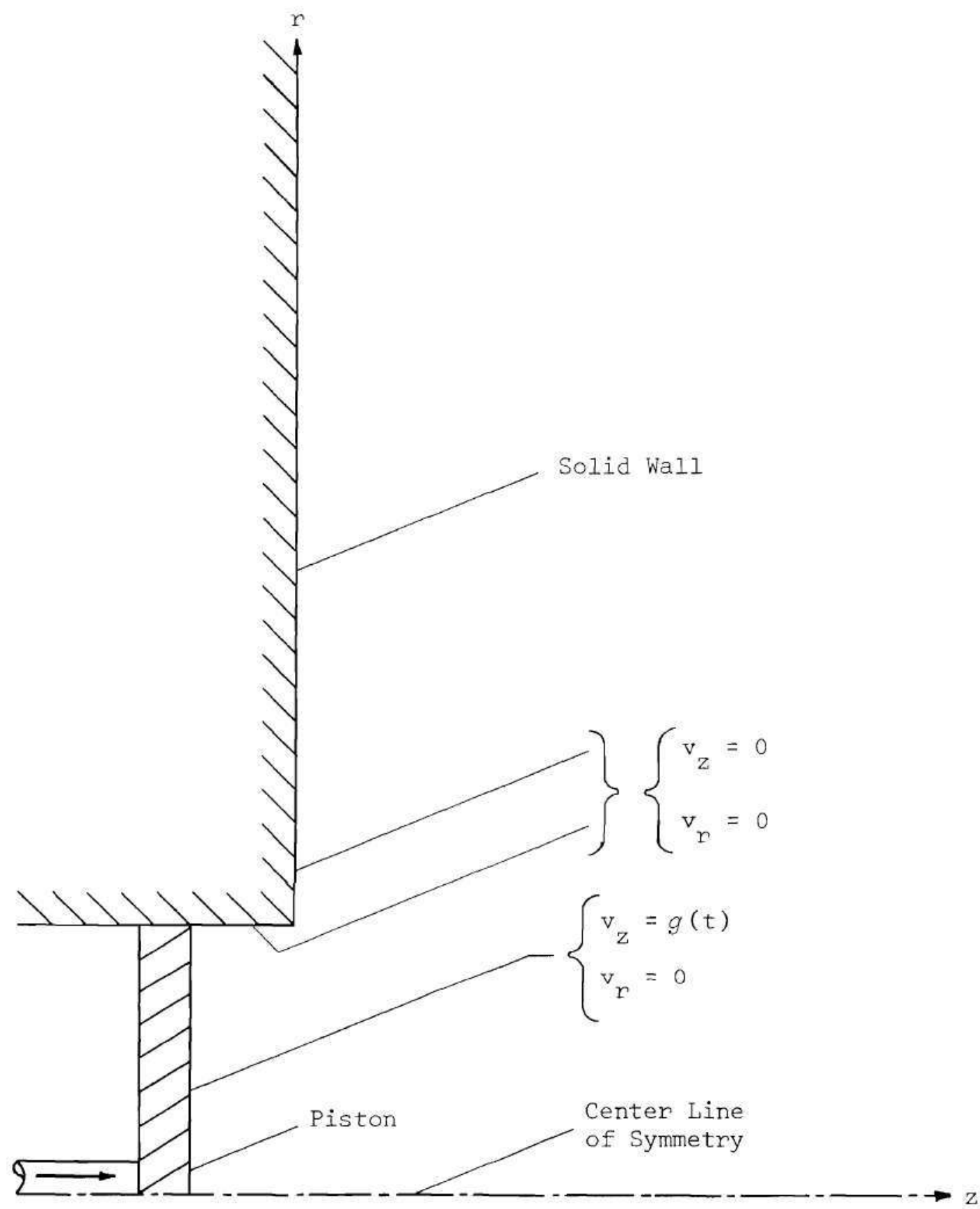

Figure 63. Proposed Geometry for Recommended Vortex Study 
of the no-slip condition, the radial velocity is zero on the piston and both velocity components vanish on the walls of the tube and on the solid boundary. Although there may arise some minor difficulties in specifying conditions on the moving boundary of the piston, the methods given in this investigation should serve to establish all other boundary values. Using this approach, the velocity distribution in the exit plane may be computed.

5. Further study of the numerical model will be necessary to answer two important questions:

a. Does there exist a time after which the vortex "forgets" how it was formed? If this is the case and the time is relatively short, then the details of the vortex formation will not be necessary for the understanding of the fully-developed vortex, and some simple similarity parameter may largely determine the structure of the vortex. The existence of a similarity parameter is necessary in order to predict the behavior of a number of vortices from one set of numerical results.

b. How far away from the vortex center is it necessary to go in order to be able to replace the velocity distribution about a viscous vortex by the velocity distribution about a vortex filament? This analogy is ultimately necessary for the useful application of the results of a viscous vortex ring to the problems of low-speed aerodynamics.

6. Finally and probably most important, the numerical results of this study must be verified experimentally. Once the model has been experimentally verified, then the 
way may be clear to investigate more difficult problems and to apply classical vortex methods to these problems. 
APPENDIX 


\title{
APPENDIX A
}

\author{
ATTEMPTED SOLUTIONS OF THE SYSTEM \\ COMPRISED OF THE VORTICITY TRANSPORT EQUATION,
}

THE DEFINITION OF VORTICITY EQUATION,

AND THE CONTINUITY EQUATION

In this appendix will be discussed a number of unsuccessful attempts to solve the system of equations composed of the vorticity transport equation, the definition of vorticity equation, and the continuity equation. The three dependent variables of the system are the vorticity and the two velocity components. As stated in the text, the major difficulty lay in the fact that the boundary condition for the axial velocity at the center line could not be firmly established.

For simplicity the differential equations will be considered in the dimensionless $\mathrm{z}, \mathrm{r}$ plane thereby avoiding any additional difficulty brought about by the transformation of spatial co-ordinates. The equations are the vorticity transport equation (the VTE),

$$
\frac{\partial \omega}{\partial t}+\frac{\partial\left(v_{z} \omega\right)}{\partial z}+\frac{\partial\left(v_{r} \omega\right)}{\partial r}=\frac{1}{R e y}\left\{\frac{\partial^{2} \omega}{\partial z^{2}}+\frac{1}{r} \frac{\partial \omega}{\partial r}-\frac{\omega}{r^{2}}+\frac{\partial^{2} \omega}{\partial r^{2}}\right\}
$$

the definition of vorticity equation (the DVE),

$$
w=\frac{\partial v_{r}}{\partial z}-\frac{\partial v_{z}}{\partial r}
$$


and the continuity equation,

$$
\frac{\partial v_{z}}{\partial z}+\frac{v_{r}}{r}+\frac{\partial v_{r}}{\partial r}=0
$$

The vorticity transport equation is derived in Chapter II. Although the introduction of the vorticity appears to be a complication, in reality it is a simplification. The system remains that of three equations in the three unknowns $\omega, v_{z}$, and $v_{r}$. However, with the introduction of the vorticity, only one of these equations is parabolic and the difficulties concerned with numerical stability affect only this equation. The remaining two equations are elliptic.

It is here assumed that the vorticity distribution may be calculated at the new time $(n+1) \delta t$ from the VTE by the methods discussed in Chapter III. The problem of this appendix is to find $v_{r}$ and $v_{z}$ which correspond to this vorticity distribution.

By combining Equations $(A-2)$ and $(A-3)$, one velocity may be eliminated from the resulting equation and the other will then be a function of geometry and some derivative of the vorticity. The best velocity for which to solve is the radial velocity $v_{r}$ since this velocity vanishes on all the boundaries. Taking the derivative of Equation (A-2) with respect to $\mathrm{z}$ gives

$$
\frac{\partial^{2} v_{r}}{\partial z^{2}}-\frac{\partial^{2} v_{z}}{\partial r \partial z}=\frac{\partial \omega}{\partial z}
$$

and taking the derivative of Equation (A-3) with respect to $r$ effects 


$$
\frac{\partial^{2} v_{z}}{\partial z \partial r}+\frac{1}{r} \frac{\partial v_{r}}{\partial r}-\frac{v_{r}}{r^{2}}+\frac{\partial^{2} v_{r}}{\partial r^{2}}=0
$$

Adding these equations together yields

$$
\frac{\partial^{2} v_{r}}{\partial z^{2}}+\frac{1}{r} \frac{\partial v_{r}}{\partial r}-\frac{v_{r}}{r^{2}}+\frac{\partial^{2} v_{r}}{\partial r^{2}}=\frac{\partial \omega}{\partial z}
$$

whose finite difference analog may be easily solved over the interior mesh by the method of successive over-relaxation given in Appendix G. Now the axial velocity component $v_{z}$ must be ascertained. No matter what finite difference equation is chosen to perform this function, the truncation error of each term should be consistent with those arising in the calculation of $\omega$. This consideration dictates the use of the central difference formulas of Appendix C. In the next paragraphs will be discussed some of the methods which were studied to calculate the axial velocity $v_{z}$ once the radial velocity $v_{r}$ was known.

\section{Method I}

The continuity equation was transformed to an integral form giving

$$
v_{z}(z, r)=-\int_{0}^{z}\left(\frac{\partial v_{r}}{\partial r}+\frac{v_{r}}{r}\right) d z
$$

A simple numerical integration of this equation is not too accurate since the integrand can be evaluated only at discrete points. Also, 
for $z$ at the first mesh point, the integration is done by the trapezoidal rule. Although a curve fit is possible at this point, accuracy is not enhanced a great deal. It is also possible to employ a polynomial curve fit to the integrand and then to integrate the polynomial. However, for a large number of mesh points in the axial direction, being able to attain good accuracy is again the problem.

\section{Method II}

Either the continuity equation or the definition of vorticity may be witten as a finite difference equation using central differences to determine the axial velocity over the interior of the mesh, but only the continuity equation may be employed at the center line since all terms of the vorticity are identically zero there. Consider the simplest of these equations for the interior of the mesh first. If the definition of vorticity is written in terms of the central differences of Appendix c, the radial velocity may be calculated as

$$
v_{z}(i, j+1)=v_{z}(i, j-1)+v_{r}(i+1, j)-v_{r}(i-1, j)-2 h w(i, j)
$$

where $\mathrm{h}$ is the mesh spacing for a square grid. Unfortunately, the axial velocity can be calculated only at every other mesh point even if the starting points are known, and they are not. Due to symmetry

$$
v_{z}(i, 1)=v_{z}(i,-1)
$$

and both are unknown. Also, the velocity at the center line is unknown 
so $v_{z}$ at the even values of the index $j$ cannot be calculated either. Similar problems also occur in attempting to use the other boundaries as starting points and in attempting to use the continuity equation instead of the definition of vorticity as described above. Even if boundary conditions could be calculated, there appears to be no method which is theoretically sound to calculate interior velocities which will then satisfy both continuity and the definition of vorticity. At the center line, only the continuity equation

$$
\frac{\partial v_{z}}{\partial z}+\frac{v_{r}}{r}+\frac{\partial v_{r}}{\partial r}=0
$$

can divulge any new information. Unfortunately in this equation at $r=0$ a singular point occurs which cannot be reconciled by evaluating its limit since the derivatives of $v_{r}$ with respect to $r$ at $r=0$ are finite and unknown.

\section{Method III}

As already seen, the greatest difficulty arising in the velocity calculations lies in determining the axial velocity at the center line. Even when this boundary value can be established (for example, by knowing the solution from calculations performed with the stream function), other problems arise. As already mentioned, finite difference analogs to the continuity and vorticity transport equations do not seem satisfactory. A new approach was attempted. The radial velocity component was calculated by Equation $(\mathrm{A}-4)$. In a similar manner, an equation may 
be found for $v_{z}$. The derivative with respect to $r$ of Equation $(A-2)$ is

$$
\frac{\partial^{2} v_{r}}{\partial z \partial r}-\frac{\partial^{2} v_{z}}{\partial r^{2}}=\frac{\partial \omega}{\partial r}
$$

and the derivative with respect to $z$ of Equation $(A-3)$ gives

$$
\frac{\partial^{2} v_{z}}{\partial z^{2}}+\frac{1}{r} \frac{\partial v_{r}}{\partial z}+\frac{\partial^{2} v_{r}}{\partial r \partial z}=0
$$

Taking the difference between these two equations and noting from the definition of vorticity that

$$
\frac{\partial v_{r}}{\partial z}=\omega+\frac{\partial v_{z}}{\partial r}
$$

gives

$$
\frac{\partial^{2} v_{z}}{\partial z^{2}}+\frac{l}{r} \frac{\partial v_{z}}{\partial r}+\frac{\partial^{2} v_{z}}{\partial r^{2}}=-\frac{\omega}{r}-\frac{\partial \omega}{\partial r}
$$

This equation can be easily solved by the method of successive overrelaxation. Again, the test of the approximation is to calculate $\mathrm{v}_{r}$ and $v_{z}$ from Equations $(A-4)$ and $(A-5)$, respectively. These should satisfy the original Equations $(A-2)$ and $(A-3)$. Evidently the method of combining equations gives a poor representation for both the DVE and the continuity equation, because the numerical results were not 
satisfactorily accurate.

\section{Concluding Comments}

In the studies preliminary to this investigation, various attempts were made to find the velocities without resorting to the stream function. The most difficult problem proved to be establishing the boundary value of the axial velocity at the center line of symmetry. Next, no methods which were theoretically sound could be found by which the velocities could be calculated from the finite difference approximations to the continuity equation and the vorticity transport equation. Finally, without resorting to the stream function, no method could be found, at least for a reasonable number of mesh points, to calculate the velocities from equations such as Equations $(A-4)$ and $(A-5)$ which were derived by manipulating the basic equations.

It appears that the problem of accurately calculating velocities without the use of the stream function will have to be solved before more difficult solutions to the Navier-Stokes equations may be obtained. For example, in three-dimensional flow or in two-dimensional, unsteady, compressible flow, the stream function does not exist. In addition, some method will have to be developed whereby boundary values may be ascertained on a line of symmetry. 


\section{APPENDIX B}

\section{DERIVATION OF THE PRESSURE EQUATION}

In the development of the governing equations used in this research, the elimination of the pressure as one of the unknowns has afforded a great simplification. Once the vorticity, stream function, and velocities are found as functions of time and space by solution of the simplified equations (the equations devoid of the pressure term), the pressure may be calculated independently at any given time.

On the other hand, simultaneous calculation of the unknown pressure and the other dependent variables may serve a useful end especially in the calculation of the flow about an obstacle which is embedded in the flow field. If the boundary conditions on the obstacle are expressed as Neumann conditions as established by the velocity components on the obstacle, no difficulties arise. However, if the boundary conditions on the obstacle are expressed as Dirichlet conditions, a new unknown, the stream function on the obstacle, is introduced. To determine this unknown function at any time, an additional equation is provided by the fact that the cyclic integral of the pressure (a physically-measurable and therefore single-valued function) must vanish along a path surrounding the obstacle. An excellent discussion of this point is given by Pearson [32].

In the problem of this research, there is no need for the simultaneous calculation of the pressure and the other dependent variables, 
and it is the purpose of this appendix to demonstrate how the pressure may be computed independently of the other variables once the vorticity, stream function, and velocities are known. This computation may be performed in a number of ways. For example, the pressure may be determined from either one of the Navier-Stokes equations in which it is now the only unknown; it need not be found by anything so complicated as a stepping procedure in time since it is now dependent upon only the spatial and temporal derivatives of known functions. Use of the Navier-Stokes equations presents an unnecessary difficulty, however. In order to evaluate the unsteady terms of these equations by finite difference techniques, the velocities at the time steps immediately preceding and succeeding the time of interest must be used. For example, one unsteady term may be evaluated in terms of the finite difference approximations of Appendix C as

$$
\frac{\partial v_{z}}{\partial t}=\frac{v_{z}^{n+1}(i, j)-v_{z}^{n-1}(i, j)}{2 \delta t}
$$

where $\mathrm{n}$ is the time index and $\delta t$ is the time step increment. This added complexity may be avoided.

In this appendix will be derived an equation from which the pressure distribution throughout the flow field may be calculated at any time $t$ in terms of only the stream function distribution at that particular time t. 


\section{The Derivation}

The derivation commences with a statement of the dimensionless governing equations (the Navier-Stokes equations and the continuity equation) which may be written as

$$
\begin{gathered}
\frac{\partial v_{z}}{\partial t}+v_{z} \frac{\partial v_{z}}{\partial z}+v_{r} \frac{\partial v_{z}}{\partial r}=-\frac{\partial p}{\partial z}+\frac{1}{R e y}\left\{\frac{\partial^{2} v_{z}}{\partial z^{2}}+\frac{1}{r} \frac{\partial v_{z}}{\partial r}+\frac{\partial^{2} v_{z}}{\partial r^{2}}\right\} \\
\frac{\partial v_{r}}{\partial t}+v_{z} \frac{\partial v_{r}}{\partial z}+v_{r} \frac{\partial v_{r}}{\partial r}=-\frac{\partial p}{\partial r}+\frac{1}{R e y}\left\{\frac{\partial^{2} v_{r}}{\partial z^{2}}+\frac{1}{r} \frac{\partial v_{r}}{\partial r}-\frac{v_{r}}{r^{2}}+\frac{\partial^{2} v_{r}}{\partial r^{2}}\right\} \\
\frac{\partial v_{z}}{\partial z}+\frac{v_{r}}{r}+\frac{\partial v_{r}}{\partial r}=0
\end{gathered}
$$

Here, the pressure has been non-dimensionalized by the term $\rho^{*} \mathrm{u}^{2}$. Differentiating Equation ( $B-1$ ) with respect to $\mathrm{z}$ and Equation $(\mathrm{B}-2)$ with respect to $r$, adding the resulting equations, and simplifying yields the expression

$$
\begin{aligned}
& \frac{\partial \beta}{\partial t}+v_{z} \frac{\partial \beta}{\partial z}+v_{r} \frac{\partial \beta}{\partial r}+\left\{\left(\frac{\partial v_{z}}{\partial z}\right)^{2}+2\left(\frac{\partial v_{r}}{\partial z}\right)\left(\frac{\partial v_{z}}{\partial r}\right)+\left(\frac{\partial v_{r}}{\partial r}\right)^{2}\right\}= \\
& -\frac{\partial^{2} p}{\partial z^{2}}-\frac{\partial^{2} p}{\partial r^{2}}+\frac{1}{R e y}\left\{\frac{1}{r} \frac{\partial \beta}{\partial r}+\frac{\partial^{2} \beta}{\partial z^{2}}+\frac{\partial^{2} \beta}{\partial r^{2}}-\frac{2}{r^{2}} \frac{\partial v_{r}}{\partial r}+2 \frac{v_{r}}{r^{3}}\right\}
\end{aligned}
$$

where, here 


$$
\beta \triangleq \frac{\partial v_{z}}{\partial z}+\frac{\partial v_{r}}{\partial r}
$$

It can be seen from the continuity equation, Equation (B-3), that

$$
\beta=-\frac{v_{r}}{r}
$$

Substituting this value of $\beta$ into Equation (B-4), performing the differentiation implied, and simplifying produces

$$
\begin{gathered}
\left(-\frac{l}{r}\right)\left(\frac{\partial v_{r}}{\partial t}+v_{z} \frac{\partial v_{r}}{\partial z}+v_{r} \frac{\partial v_{r}}{\partial r}-\frac{1}{R e y}\left[\frac{\partial^{2} v_{r}}{\partial z^{2}}+\frac{1}{r} \frac{\partial v_{r}}{\partial r}-\frac{v_{r}}{r^{2}}+\frac{\partial^{2} v_{r}}{\partial z^{2}}\right]\right)=(B-6) \\
-\frac{\partial^{2} p}{\partial z^{2}}-\frac{\partial 2 p}{\partial r^{2}}-\left\{\left(\frac{\partial v_{z}}{\partial z}\right)^{2}+2\left(\frac{\partial v_{z}}{\partial r}\right)\left(\frac{\partial v_{r}}{\partial z}\right)+\left(\frac{\partial v_{r}}{\partial r}\right)^{2}+\left(\frac{v_{r}}{r}\right)^{2}\right\}
\end{gathered}
$$

Study of the second of the Navier-Stokes equations, Equation (B-2), reveals the bracketed term on the left-hand side of Equation (B-6) to be nothing other than the term

$$
\frac{\partial p}{\partial r}
$$

which when substituted into Equation (B-6) allows the derivation of an equation relating the pressure distribution to the velocity distributions, 


$$
\frac{\partial^{2} p}{\partial z^{2}}+\frac{1}{r} \frac{\partial p}{\partial r}+\frac{\partial^{2} p}{\partial z^{2}}=-2\left\{\left(\frac{\partial v_{z}}{\partial r}\right)\left(\frac{\partial v_{r}}{\partial z}\right)-\left(\frac{\partial v_{z}}{\partial z}\right)\left(\frac{\partial v_{r}}{\partial r}\right)+\left(\frac{v_{r}}{r}\right)^{2}\right\}
$$

Note here that although the velocities are functions of time, all time derivatives have vanished; only the velocities at the particular time of interest are needed. When the velocities are expressed in terms of the stream function $\psi$, the pressure equation takes the final form

$$
\begin{gathered}
\frac{\partial^{2} p}{\partial z^{2}}+\frac{1}{r} \frac{\partial p}{\partial r}+\frac{\partial^{2} p}{\partial r^{2}}=-\frac{2}{r^{2}}\left\{\frac{1}{r^{2}}\left(\frac{\partial^{2} \psi}{\partial z^{2}}\right)\right. \\
\left.+\left(\frac{\partial^{2} \psi}{\partial z^{2}}\right)\left(\frac{1}{r} \frac{\partial \psi}{\partial r}-\frac{\partial^{2} \psi}{\partial r^{2}}\right)-\left(\frac{\partial^{2} \psi}{\partial z \partial r}\right)\left(\frac{1}{r} \frac{\partial \psi}{\partial z}-\frac{\partial^{2} \psi}{\partial z \partial r}\right)\right\}
\end{gathered}
$$

This expression allows the computation of the pressure at any particular time $t$ in terms of only the stream function distribution at that same time.

Equation $(B-8)$ is an elliptic, linear, partial differential equation requiring boundary conditions on the region of interest. If the pressure can be expressed on the boundaries, the Dirichlet problem results, and if the normal derivative of the pressure can be expressed on the boundaries, the Neumann problem results. In this appendix, the pressure equation will be solved using mixed boundary conditions. This is the way that the DVE is solved. Consider now the boundary conditions. 
Boundary Condition for Large $z$

The dimensional pressure at a great distance from the origin is specified as the reference pressure, $P_{R}^{*}$, which for this case takes the value of atmospheric pressure. Hence, in terms of the dimensionless pressure,

$$
\text { for large } \mathrm{z} \quad \mathrm{p}=\frac{\mathrm{P}_{\mathrm{R}}^{*}}{\rho \mathrm{v}^{2}}
$$

Boundary Condition for Large $r$

Once again the dimensional pressure is specified as atmospheric. Hence, dimensionlessly,

$$
\text { for large } r \quad p=\frac{P_{R}^{*}}{\rho * U^{2}}
$$

Boundary Condition at $r=0$

At the center line due to symmetry

$$
\frac{\partial p}{\partial r}=0
$$

Boundary Condition at $z=0$

The boundary condition on pressure both in the exit plane and on the solid boundary may be determined by investigating the governing equations. Differentiating the continuity equation, Equation (B-3), with respect to $z$, substituting the result into the axial momentum 
equation, Equation (B-1), and simplifying reveals that

$$
\begin{gathered}
\frac{\partial v_{z}}{\partial t}+v_{z} \frac{\partial v_{z}}{\partial z}+v_{r} \frac{\partial v_{z}}{\partial r}=-\frac{\partial p}{\partial z}+\frac{1}{R e y}\left\{-\frac{1}{r} \frac{\partial v_{r}}{\partial z}-\frac{\partial^{2} v_{z}}{\partial z \partial r}\right. \\
\left.+\frac{1}{r} \frac{\partial v_{z}}{\partial r}+\frac{\partial^{2} v_{z}}{\partial r^{2}}\right\}
\end{gathered}
$$

Employing the definition of vorticity given by

$$
\omega=\frac{\partial v_{r}}{\partial z}-\frac{\partial v_{z}}{\partial r}
$$

allows the simplification of Equation (B-12) to

$$
\frac{\partial v_{z}}{\partial t}+v_{z} \frac{\partial v_{z}}{\partial z}+v_{r} \frac{\partial v_{z}}{\partial r}=-\frac{\partial p}{\partial z}-\frac{1}{R e y}\left\{\frac{\partial \omega}{\partial r}+\frac{\omega}{r}\right\}
$$

This is the equation to be used to determine the boundary condition on pressure in the plane $z=0$.

Since the plane $z=0$ is divided into two different regions, the exit plane and the solid wall, consider the exit plane first.

Because it has been specified that the radial velocity vanishes in the exit plane, Equation (B-14) simplifies to

$$
\frac{\partial v_{z}}{\partial t}+v_{z} \frac{\partial v_{z}}{\partial z}=-\frac{\partial p}{\partial z}-\frac{1}{R e y}\left\{\frac{\partial \omega}{\partial r}+\frac{\omega}{r}\right\}
$$


The axial velocity in the exit plane is an input parameter taking the form

$$
v_{z}=\frac{v_{z}^{*}}{U_{C L}} \frac{U_{C L}}{U}
$$

where

$$
\frac{v_{z}^{*}}{U_{C L}}=f(r)
$$

and

$$
\frac{{ }^{U} L}{U}=g(t)
$$

Hence,

$$
\frac{\partial v_{z}}{\partial t}=f(r) \frac{\partial g(t)}{\partial t}
$$

Since the distribution of $\mathrm{v}_{\mathrm{z}}$ and the corresponding stream function is already known, the term

$$
\left.\frac{\partial v_{z}}{\partial z}\right|_{z=0}=h(t, z=0, r)
$$

may be approximated numerically by a curve fit or a Taylor series 
expansion. The problem is analogous to that of estimating the boundary value of the vorticity necessary for the solution of the VTE. A similar technique may be applied to evaluate the term

$$
\left.\frac{\partial \omega}{\partial r}\right|_{z=0}=k(t, z=0, r)
$$

Substituting Equations $(B-16),(B-17)$, and (B-18) into Equation (B-15) then yields the Neumann condition for pressure in the exit plane as

$$
\begin{gathered}
\text { at } z=0, \quad r \leq 1 \\
\frac{\partial p}{\partial z}=-\left\{f(r) \frac{\partial g(t)}{\partial t}+f(r) g(t) h(t, z=0, r)\right\} \\
-\frac{1}{R e y}\left\{k(t, z=0, r)+\frac{\omega}{r}\right\}
\end{gathered}
$$

On the solid boundary where the axial velocity vanishes, Equation (B-15) simplifies to show that

$$
\begin{gathered}
\text { at } z=0, \quad r>1 \\
\frac{\partial p}{\partial z}=-\frac{1}{\operatorname{Rey}}\left\{k(t, z=0, r)+\frac{\omega}{r}\right\}
\end{gathered}
$$

The pressure distribution may now be calculated at any time $t$ at which the corresponding stream function and vorticity distributions 
are known by using Equation ( $B-8$ ) subject to the boundary conditions stated in Equations $(B-9),(B-10),(B-11),(B-19)$, and $(B-20)$. Although the pressure equation is expressed in terms of the stream function only, some of the boundary conditions are functions of vorticity. Introduction of the vorticity on the boundaries presents no great difficulty, but remember that the vorticity may be expressed in terms of the stream function if this is desirable.

As in the case of the VTE and the DVE, the pressure equation and its boundary conditions must be transformed to the $\xi, n$ plane which is simulated by a square mesh of $(M+1)^{2}$ equally spaced grid points. The transformed equation and the accompanying boundary conditions must then be approximated by finite difference equations. The application of the finite difference expression for the governing equation, Equation (B-8), to each interion mesh point gives a system of simultaneous algebraic equations. Since the boundary values of pressure are also unknown, additional equations must be developed from the boundary conditions. The end result is a system of $(M+1)^{2}$ simultaneous algebraic equations in the $(M+1)^{2}$ unknowns, i.e., the values of the dependent variable at each grid point. The transformation of the governing equation and the derivation of the finite difference analog are not included here because both of these are straightforward derivations. The transformation of Equation $(B-8)$ is the same as the transformation of the VTE or the DVE. The derivation of the finite difference equation follows closely the development of Appendix $\mathrm{F}$ wherein the DVE--an equation similar to the equation which results from the transformation of Equation ( $B-8)--$ is written as a finite difference equation. 


\section{Suggestions for the Boundary Conditions}

The difficulties inherent in the solution of the pressure equation are easily recognized. They are concerned with the numerical approximations which are needed to evaluate the boundary conditions indicated in Equations $(B-9),(B-10),(B-11),(B-19)$, and $(B-20)$. The method of trial and error will probably determine the most accurate and easiest way to numerically approximate the boundary conditions. However, in order to facilitate any future study of the calculation of the pressure through the use of Equation (B-8), a few suggestions will be made here regarding the evaluation of the boundary conditions. The following discussion will describe approximations which are primarily those of the finite difference technique, and the reader may wish to refer to Appendix C for the development of the finite difference method. Since the solution of the pressure equation must be found in the transformed plane, the derivatives of interest will be transformed to the $\xi, n$ plane. The finite difference mesh of the transformed plane is composed of $\mathrm{M}+1$ grid points in both the axial and radial directions, and the distance between mesh points is given by

$$
\mathrm{h}=\frac{1}{\mathrm{M}}
$$

The development of the boundary conditions will begin with the easiest one and then will move on to the more difficult expressions. Evaluation of the Pressure at Large Distances

This boundary condition is expressed by Equations (B-9) and 
(B-10), and it is applied simply by specifying the pressure at the grid point furthest removed from the origin. Hence,

$$
p(i, M)=p(M, j)=\frac{p_{R}^{*}}{\rho^{*} U^{2}}
$$

Evaluation of the Term $\frac{\partial p}{\partial r}=0$

This boundary condition is expressed by Equation (B-11) at the center line. The transformation of the original boundary condition is simply

$$
\frac{\partial p}{\partial r}=b(1-n) \frac{\partial p}{\partial n}=0
$$

and since $n=0$ on the center line,

$$
\frac{\partial p}{\partial r}=0
$$

implies that

$$
\frac{\partial p}{\partial \eta}=0
$$

The problem now is to represent the derivative of pressure with respect to $n$ in a suitable finite difference form.

The simplest representation is made by expressing the pressure at the grid point just inside the boundary in terms of the pressure and its 
derivatives on the boundary by means of a truncated Taylor series. Considering any axial station depicted by the index $i$ and the boundary at $n=0$ gives the expression

$$
p(i, 1)=p(i, 0)+\frac{h}{1 !} \frac{\partial p(i, 0)}{\partial \eta}+\frac{h^{2}}{2 !} \frac{\partial^{2} p(i, 0)}{\partial \eta^{2}}+\frac{h^{3}}{3 !} \frac{\partial^{3} p(i, \bar{n})}{\partial \eta^{3}}
$$

where $\mathrm{h}$ is the mesh spacing and $\bar{n}$ lies in the interval $(0, h)$. If it can now be assumed that the second derivative also vanishes at the boundary, then the boundary value is given by

$$
p(0, j)=p(I, j)
$$

with a truncation error equal to that of a central difference representation. However, for the case of a symmetric function, the assumption of a vanishing second derivative is not valid.

Since the second derivative usually is not known, Esch [22] has introduced a method to handle such problems. The pressure for the two grid points just inside the boundary may be expressed as the truncated Taylor series:

$$
\begin{aligned}
& p(1, j)=p(0, j)+\frac{h}{1 !} \frac{\partial p(0, j)}{\partial \eta}+\frac{h^{2}}{2 !} \frac{\partial^{2} p(0, j)}{\partial \eta^{2}}+\frac{h^{3}}{3 !} \frac{\partial^{3} p\left(0, n_{1}\right)}{\partial \eta^{3}} \\
& p(2, j)=p(0, j)+\frac{2 h}{1 !} \frac{\partial p(0, j)}{\partial \eta}+\frac{(2 h)^{2}}{2 !} \frac{\partial^{2} p(0, j)}{\partial \eta^{2}}+\frac{(2 h)^{3}}{3 !} \frac{\partial^{3} p\left(0, n_{2}\right)}{\partial \eta^{3}}
\end{aligned}
$$


where $\mathrm{h}$ is the mesh spacing, $n_{1}$ lies in the interval $(0, \mathrm{~h})$, and $n_{2}$ lies in the interval $(0,2 \mathrm{~h})$. Eliminating the unknown second derivative between these two equations, and simplifying the result by the fact that the first derivative vanishes gives

$$
p(0, j)=\frac{1}{3}\{4 p(1, j)-p(2, j)\}+\frac{4}{9} h^{3} \frac{\partial^{3} p(i, \bar{\eta})}{\partial n^{3}}
$$

where by the mean value theorem $\bar{n}$ lies in the interval $(0,2 \mathrm{~h})$. Although the truncation error of this expression is less than that of a central difference formulation, the big significance is that this error is at least as small as the error in a central difference. The truncation error arising from the difference approximation to the boundary condition should be at least as small as the one arising from the difference expression for the governing equation.

Use of Equation (B-22) gives the additional equation necessary for the boundary grid point for which the original boundary condition applies, i.e.,

$$
\frac{\partial p}{\partial r}=0
$$

Evaluation of the Term $\frac{\partial v_{z}}{\partial z}$

This expression is found in Equation (B-17) where it must be evaluated in order to obtain the boundary value of pressure in the exit plane and on the solid boundary.

In the transformed plane this term may be written 


$$
\frac{\partial v_{z}}{\partial z}=a(1-\xi) \frac{\partial v_{z}}{\partial \xi}=a \frac{\partial v_{z}}{\partial \xi}
$$

The axial velocity at the grid points located at $i=1$ and $i=2$ may be expressed as truncated Taylor series about $i=0$ for any radial station denoted by the index $j$. These expressions are:

$$
\begin{aligned}
& v_{z}(1, j)=v_{z}(0, j)+\frac{h}{1 !} \frac{\partial v_{z}(0, j)}{\partial \xi}+\frac{h^{2}}{2 !} \frac{\partial^{2} v_{z}(0, j)}{\partial \xi^{2}}+\frac{h^{3}}{3 !} \frac{\partial^{3} v_{z}\left(\xi_{I}, j\right)}{\partial \xi^{3}} \\
& v_{z}(2, j)=v_{z}(0, j)+\frac{(2 h)}{1 !} \frac{\partial v_{z}(0, j)}{\partial \xi}+\frac{(2 h)^{2}}{2 !} \frac{\partial^{2} v_{z}(0, j)}{\partial \xi^{2}}+\frac{(2 h)^{3}}{3 !} \frac{\partial^{3} v_{z}(\xi, j)}{\partial \xi^{3}}
\end{aligned}
$$

where $\xi_{1}$ lies in the interval $(0, h)$ and $\xi_{2}$ lies in the interval $(0,2 \mathrm{~h})$. Eliminating the second derivative between these two expressions gives

$$
\frac{\partial v_{z}(0, j)}{\partial \xi}=\frac{1}{2 h}\left\{-3 v_{z}(0, j)+4 v_{z}(1, j)-v_{z}(2, j)\right\}+\frac{1}{3} h^{2} \frac{\partial^{3} v_{z}(\bar{\xi}, j)}{\partial \xi^{3}}(B-23)
$$

where $\bar{\xi}$ lies in the interval $(0,2 \mathrm{~h})$. The truncation error of this approximation is of the same order of magnitude as the error of a central difference expression.

The velocities $v_{z}(0, j), v_{z}(l, j)$, and $v_{z}(2, j)$ must now be determined. The first of these may be obtained from the given parameter

$$
\mathrm{v}_{\mathrm{z}}(0, r)=f(r) g(t)
$$


in the exit plane. On the solid boundary

$$
v_{z}(0, j)=0
$$

from the no-slip condition. The other velocities may be easily calculated from the known stream function distribution by the equation

$$
v_{z}(\xi, n)=\frac{b(1-n)}{x} \frac{\partial \psi}{\partial n}
$$

whose finite difference representation is

$$
v_{z}(i, j)=\frac{b(1-j h)}{x} \frac{\psi(i, j+1)-\psi(i, j-1)}{2 h}
$$

The term $\frac{\partial v_{z}(0, j)}{\partial z}$ may now be calculated by substituting Equations (B-24) and (B-25) into Equation (B-23). The truncation errors of all these approximations are of order $h^{2}$, and since these results are added in Equation ( $B-23)$, the truncation error of Equation $(B-23)$ is of order $h^{2}$.

Another method of evaluating the axial derivative of the axial velocity might be to express this derivative in terms of the stream function as

$$
\frac{\partial v_{z}}{\partial z}=a(1-\xi) \frac{\partial v_{z}}{\partial \xi}=\frac{a b(1-\xi)(1-n)}{x} \frac{\partial^{2} \psi}{\partial \xi \partial \eta}
$$

However, this involves the approximation of a mixed derivative which 
might prove difficult on the boundary. Evaluation of the Term $\frac{\partial \omega}{\partial r}$

The transformation of this expression gives

$$
\frac{\partial \omega}{\partial r}=b(1-\eta) \frac{\partial \omega}{\partial \eta}
$$

and this may be evaluated by a central difference to yield

$$
\frac{\partial \omega}{\partial r}=b(1-j h) \frac{\omega(i, j+1)-\omega(i, j-1)}{2 h}
$$

which has the same truncation error as the other boundary conditions. The reader may have noticed that the term $\frac{\omega}{r}$ was not discussed. This term can cause some difficulty if it is needed at the center line where $r$ vanishes. However, it is not needed at the center line. When the finite difference approximation to the pressure equation is derived using central differences, the pressure is expressed in terms of variables of four adjacent points, that is, parameters evaluated at the grid points above, below, to the right and to the left of the central point $i, j$. When this mesh point corresponds to the values of $i=1$ and $j=1$, the only boundary conditions needed are for the points $i=0, j=1$ and $i=1, j=0$. Hence, no boundary condition need be evaluated at the point $i=0, j=0$ on the center line. This line of reasoning also applies to the other three corners of the mesh. Since the corner mesh points do not enter into the calculations, the system of simultaneous algebraic equations which is solved is really composed 
of $(M+1)^{2}-4$ equations.

In this appendix, an equation has been derived by which the pressure may be calculated independently at any particular time for which the vorticity and stream function distributions have already been computed. The boundary conditions and some suggested finite difference approximations for them have also been discussed. 
APPENDIX C

THE FINITE DIFFERENCE TECHNIQUE

In this appendix, several different finite difference approximations are developed for the partial derivatives of interest. Of importance in this discussion is recognition of the truncation error involved in each approximation.

Consider the mesh of Figure $\mathrm{C}-1$ which is evenly spaced in both the axial and radial directions. Following the notation of the text, the spatial co-ordinates are given by $\xi$ and $n$, and the partial derivatives are evaluated at the various grid points $(i, j)$ where

$$
\xi=\frac{i}{M} \quad n=\frac{j}{M}
$$

and $h=\frac{1}{M}$ is the mesh spacing. Both indices vary from zero to $M$.

In the following paragraphs, the general function $G$ is chosen as the dependent variable and derivatives will be taken only in the $\xi$-direction. It is to be recognized, however, that a derivative in the $n$-direction, or in the time direction for that matter, is calculated in the same manner as the ones to be derived.

\section{First Derivative--Central Difference Approximation}

The first derivative

$$
\frac{\partial G}{\partial \xi}
$$




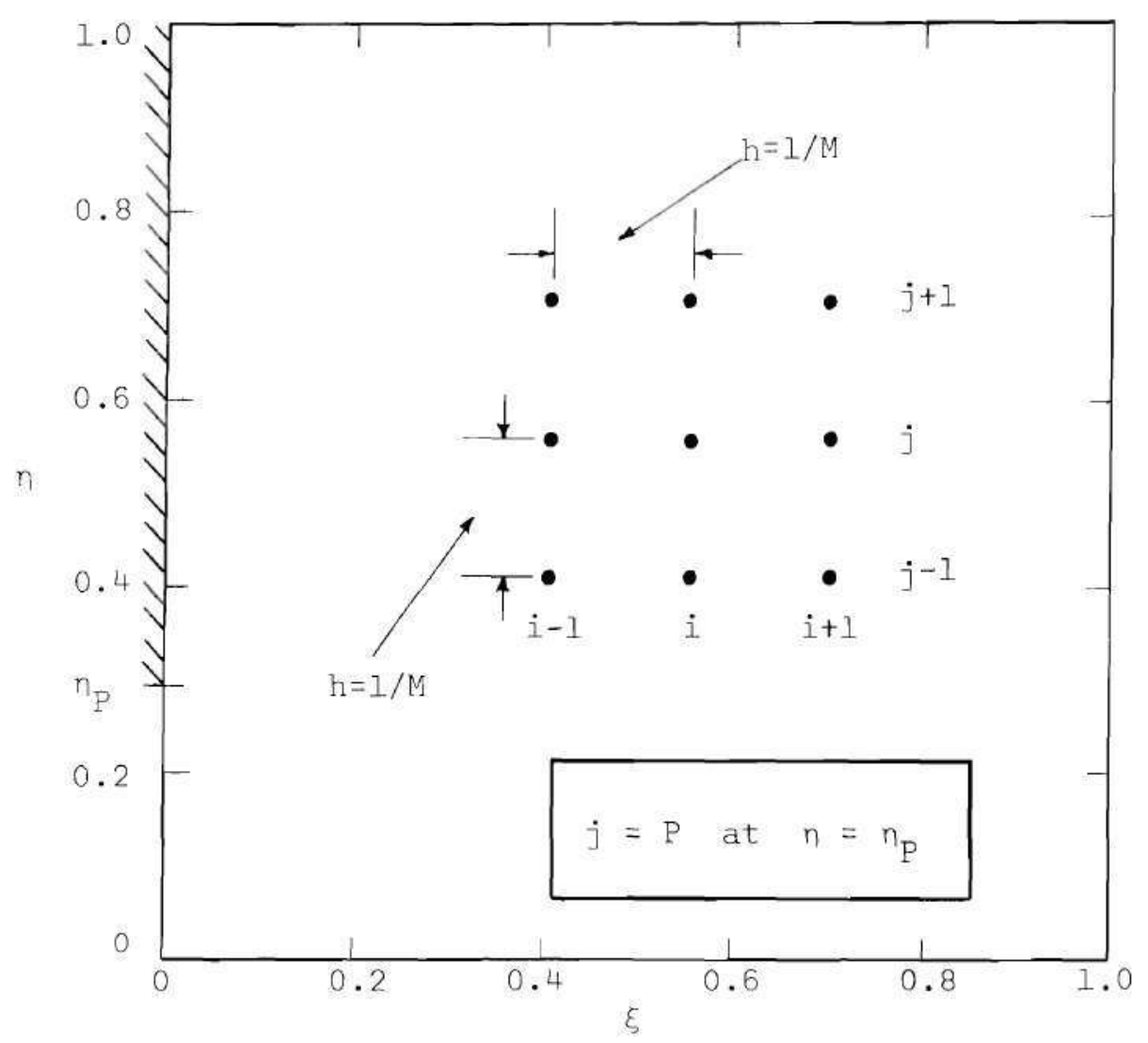

Figure C-1. Mesh Spacing in the Transformed Plane 
is evaluated at the point $(\xi, \eta)$ or the mesh point $(i, j)$. It is assumed here and throughout this investigation that higher onder derivatives exist and are finite. The value of the function $G$ at the points $(\xi+h, n)$ and $(\xi-h, n)$, the mesh points $(i+l, j)$ and $(i-l, j)$, respectively, may be approximated by using Lagrange's form of the remainder * in the truncated Taylor series expansions about the point $(\xi, n)$. Hence

$$
G(\xi+h, n)=G(\xi, n)+\frac{h}{1 !} \frac{\partial G(\xi, n)}{\partial \xi}+\frac{h^{2}}{2 !} \frac{\partial^{2} G(\xi, n)}{\partial \xi^{2}}+\frac{h^{3}}{3 !} \frac{\partial^{3} G(\xi I, n)}{\partial \xi^{3}}
$$

and

$$
G(\xi-h, n)=G(\xi, n)-\frac{h}{1 !} \frac{\partial G(\xi, n)}{\partial \xi}+\frac{h^{2}}{2 !} \frac{\partial^{2} G(\xi, n)}{\partial \xi^{2}}-\frac{h^{3}}{3 !} \frac{\partial^{3} G(\xi, n)}{\partial \xi^{3}}
$$

where $\xi_{1}$ lies in the interval $(\xi, \xi+h)$ and $\xi_{2}$ lies in the interval $(\xi-h, \xi)$. Taking the difference of these two equations yields the fact that

$$
G(\xi+h, n)-G(\xi-h, n)=2 h \frac{\partial G(\xi, n)}{\partial \xi}+\frac{h^{3}}{3 !}\left\{\frac{\partial^{3} G\left(\xi_{1}, n\right)}{\partial \xi^{3}}+\frac{\partial^{3} G\left(\xi_{2}, n\right)}{\partial \xi^{3}}\right\}
$$

which simplifies to

* See for example, Thomas, Calculus and Analytic Geometry, Addison-Wesley, Reading, Massachusetts, 1958, P. 579. 


$$
\frac{\partial G(\xi, n)}{\partial \xi}=\frac{G(\xi+h, n)-G(\xi-h, n)}{2 h}-\frac{h^{2}}{12}\left\{\frac{\partial^{3} G\left(\xi_{1}, n\right)}{\partial \xi^{3}}+\frac{\partial^{3} G\left(\xi_{2}, n\right)}{\partial \xi^{3}}\right\}
$$

Rewriting part of this in terms of the indices $i$ and $j$ shows finally that

$$
\frac{\partial G(\xi, n)}{\partial \xi}=\frac{G(i+1, j)-G(i-1, j)}{2 h}-\frac{h^{2}}{6} \frac{\partial^{3} G(\bar{\xi}, n)}{\partial \xi^{3}}
$$

where by the mean value theorem $\bar{\xi}$ lies in the interval $(\xi-h, \xi$ th). Here it can be seen that when the approximation

$$
\frac{\partial G(\xi, \eta)}{\partial \xi}=\frac{G(i+1, j)-G(i-1, j)}{2 h}
$$

is written, a term is neglected whose coefficient is of the order $h^{2}$ for $\frac{\partial^{3} G(\bar{\xi}, \eta)}{\partial \xi^{3}}$ finite. By decreasing the mesh spacing $h$, the size of this neglected term, the truncation error, is diminished.

First Derivative--Forwand Difference Approximation

In order to evaluate the first derivative

$$
\frac{\partial G(\xi, n)}{\partial \xi}
$$

the truncated Taylor series expansion for $G(\xi+h, n)$ may be written as

$$
G(\xi+h, n)=G(\xi, n)+\frac{h}{1 !} \frac{\partial G(\xi, n)}{\partial \xi}+\frac{h^{2}}{2 !} \frac{\partial^{2} G(\xi, n)}{\partial \xi^{2}}
$$


which may be rearranged to

$$
\frac{\partial G(\xi, n)}{\partial \xi}=\frac{G(\xi+h, n)-G(\xi, n)}{h}-\frac{h}{2} \frac{\partial^{2} G(\xi, n)}{\partial \xi^{2}}
$$

Rewriting part of this in terms of the indices, the derivative becomes

$$
\frac{\partial G(\xi, n)}{\partial \xi}=\frac{G(i+1, j)-G(i, j)}{h}-\frac{h}{2} \frac{\partial^{2} G\left(\xi_{1}, n\right)}{\partial \xi^{2}}
$$

Since the coefficient of the truncation error is of order $h$, it is seen that this formulation is not so accurate as the central difference.

First Derivative--Backward Difference Approximation

In a derivation analogous to the forward difference, the Taylor series expansion for $G(\xi-h, n)$ about the point $(\xi, n)$ may be written and simplified to produce a backward difference approximation which is of the form

$$
\frac{\partial G(\xi, n)}{\partial \xi}=\frac{G(i, j)-G(i-1, j)}{h}+\frac{h}{2} \frac{\partial^{2} G(\xi, n)}{\partial \xi^{2}}
$$

This has the same truncation error as the forward difference. It may now be seen that of the simple approximations of the first derivative which require evaluation of the function at only two points, the central difference approximation has the smallest truncation error and is thus preferred. 
First Derivative--Central Difference Approximation

$\underline{\text { (Unequally Spaced Grid) }}$

The analysis will depart for a moment from the equally spaced grid of Figure C-l to consider some of the difficulties associated with the unequally spaced points of Figure $\mathrm{C}-2$. To obtain an approximation for the partial derivative

$$
\frac{\partial G(\xi, n)}{\partial \xi}
$$

by evaluating the function at the unequally spaced points whose coordinates are $\left(\xi+h_{1}, n\right)$ and $\left(\xi-h_{2}, n\right)$, the Taylor series expansions may be written

$$
\begin{aligned}
& G\left(\xi+h_{1}, n\right)=G(\xi, n)+\frac{h_{1}}{1 !} \frac{\partial G(\xi, n)}{\partial \xi}+\frac{\left(h_{1}\right)^{2}}{2 !} \frac{\partial^{2} G\left(\xi_{1}, n\right)}{\partial \xi^{2}} \\
& G\left(\xi-h_{2}, n\right)=G(\xi, n)-\frac{h_{2}}{1 !} \frac{\partial G(\xi, n)}{\partial \xi}+\frac{\left(h_{2}\right)^{2}}{2 !} \frac{\partial G\left(\xi_{2}, n\right)}{\partial \xi^{2}}
\end{aligned}
$$

Eliminating the parameter $G(\xi, n)$ between these two equations produces

$$
\frac{\partial G(\xi, n)}{\partial \xi}=\frac{G\left(\xi+h_{1}, n\right)-G\left(\xi-h_{2}, n\right)}{h_{1}+h_{2}}-\frac{1}{2}\left(h_{1}-h_{2}\right) \frac{\partial^{2} G(\bar{\xi}, n)}{\partial \xi^{2}}
$$

This approximation is a little more difficult to apply than the one using equally spaced grid points because here it is necessary to keep track of the grid points in order to use the correct mesh spacings. Although the magnitude of the truncation error, $\left(h_{1}-h_{2}\right)$, may be less 


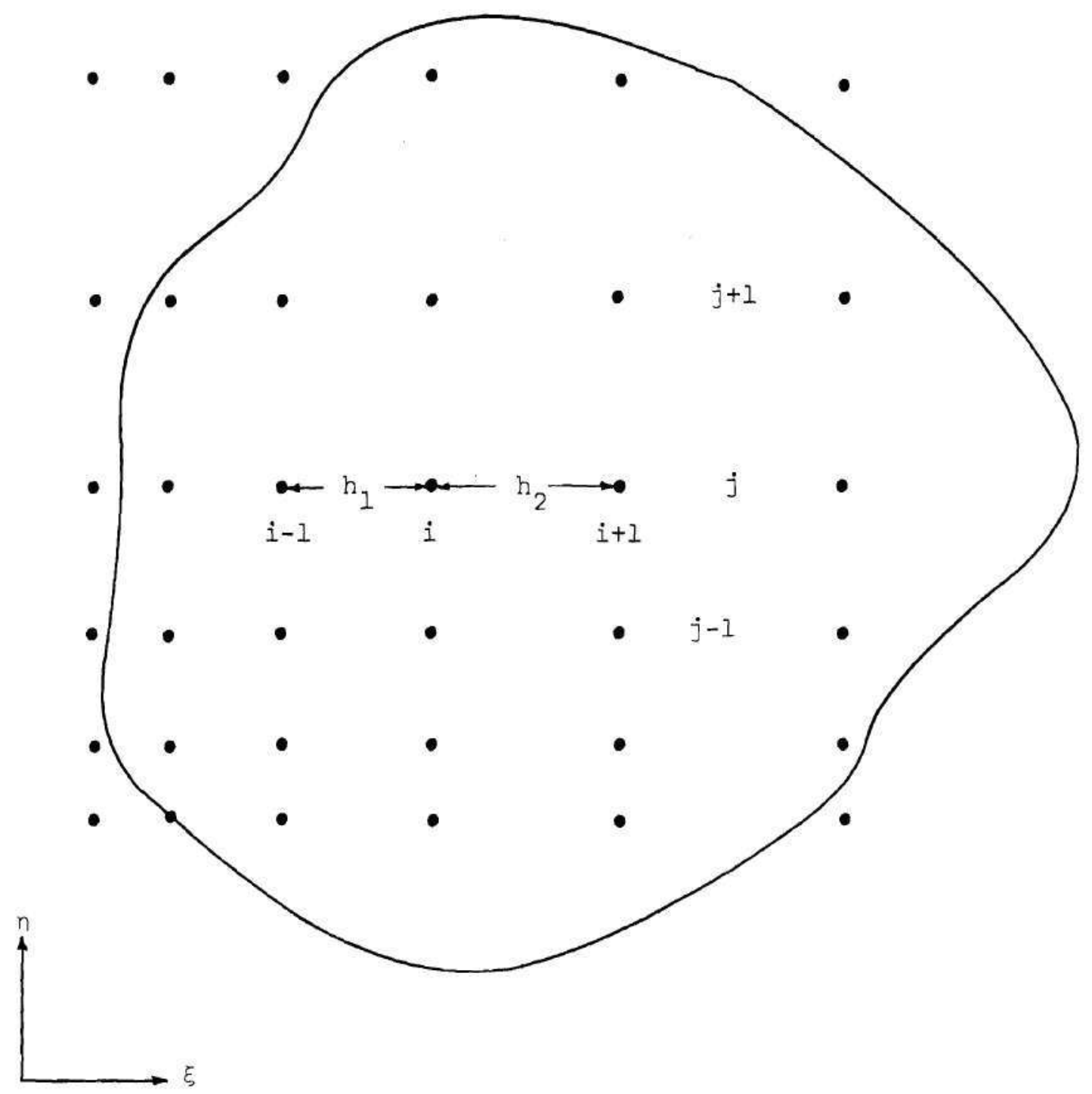

Figure C-2. Portion of Grid of Unequally Spaced Mesh Points 
than $h$, it is still higher than that of the equally spaced grid, $h^{2}$.

\section{Second Derivative--Central Difference Approximation}

Evaluation of the second derivative

$$
\frac{\partial^{2} G(\xi, n)}{\partial \xi^{2}}
$$

requires the Taylor series expansions

$G(\xi+h, n)=G(\xi, n)+\frac{h}{1 !} \frac{\partial G(\xi, n)}{\partial \xi}+\frac{h^{2}}{2 !} \frac{\partial^{2} G(\xi, n)}{\partial \xi^{2}}+\frac{h^{3}}{3 !} \frac{\partial^{3} G(\xi, n)}{\partial \xi^{3}}+\frac{h^{4}}{4 !} \frac{\partial^{4} G(\xi, n)}{\partial \xi^{4}}$

$G(\xi-h, n)=G(\xi, n)-\frac{h}{1 !} \frac{\partial G(\xi, n)}{\partial \xi}+\frac{h^{2}}{2 !} \frac{\partial^{2} G(\xi, n)}{\partial \xi^{2}}-\frac{h^{3}}{3 !} \frac{\partial^{3} G(\xi, n)}{\partial \xi^{3}}+\frac{h^{4}}{4 !} \frac{\partial^{4} G(\xi, n)}{\partial \xi^{4}}$

Addition of these two equations allows elimination of the first derivative and yields after some simplification

$$
\frac{\partial^{2} G(\xi, n)}{\partial \xi^{2}}=\frac{G(i+1, j)+G(i-1, j)-2 G(i, j)}{h^{2}}-\frac{1}{12} h^{2} \frac{\partial^{4} G(\bar{\xi}, n)}{\partial \xi^{4}}
$$

This result is noteworthy for several reasons. First, elimination of the first derivative in this central difference also results in elimination of the thind derivative, so the net effect is a truncation error of the order of $h^{2}$ instead of $h$ which would result from a forward on backward difference approximation. Second, the order of magnitude of the truncation error for this approximation is the same as that for the 
central difference of the first derivative--the errors of the two approximations are consistent. Thus, if a second order equation is written entirely in terms of central differences, the order of magnitude of each of the individual truncation errors will be the same.

It is worth noting that difference approximations offering smaller truncation errors may be derived at the expense of complexity by writing Taylor series expansions for a langer number of grid points. For example, a five-point central difference expression for a second derivative may be written as

$$
\begin{gathered}
\frac{\partial^{2} G(\xi, n)}{\partial \xi^{2}}=\frac{1}{12 h^{2}}\{-G(i-2, j)+16 G(i-1, j)-30 G(i, j)+ \\
16 G(i+1, j)-G(i+2, j)\}+\frac{h^{4}}{90} \frac{\partial^{6} G(\bar{\xi}, n)}{\partial \xi^{6}}
\end{gathered}
$$

where $\bar{\xi}$ lies in the interval $(\xi-2 h, \xi+2 h)$. Although the truncation error is smaller here than for the corresponding two-point formula, an additional difficulty occurs when this approximation is applied near the boundary. This case is illustrated in Figure C-3. One of the grid points falls outside the boundary, and somehow this problem must be reconciled.

A few words may also be said in defense of forward and backward differences. When the boundary of Figure $\mathrm{C}-3$ is again considered, the investigator may prefer a forward difference which offers the same order of truncation error as the two-point central difference formula. Although such a formulation requires evaluation of the function at more 


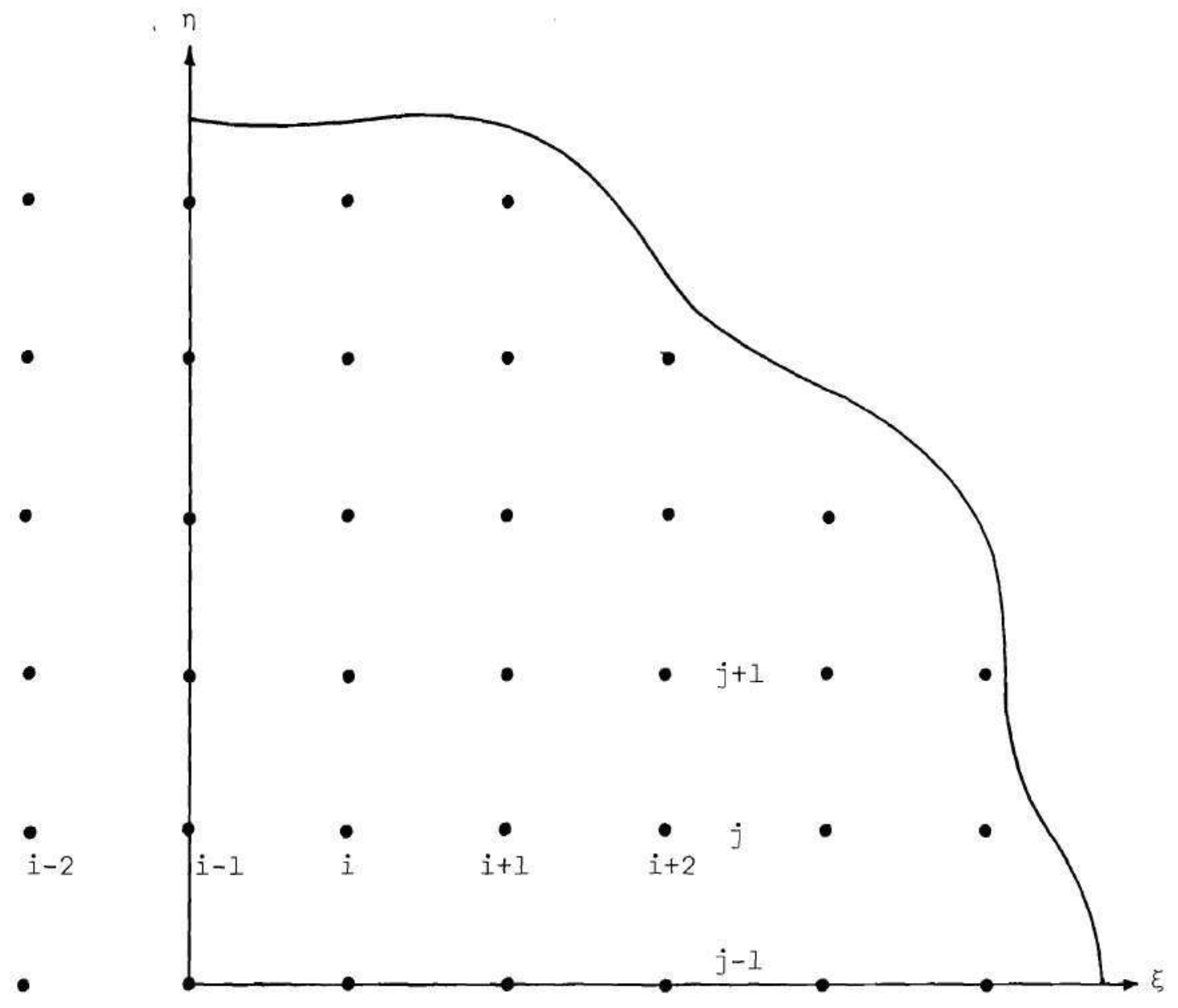

Figure C-3. Illustration of Use of Five-Point Formula Near a Boundary 
than two grid points, there is no need to consider a mesh point outside the boundary. 


\section{APPENDIX D}

\section{STABILITY ANALYSIS OF THE VTE}

It is the purpose of a stability analysis to find a finite difference approximation to a partial differential equation for which the numerical error--the difference between the numerical solution of the finite difference equation and the exact solution of the difference equation--is small throughout the region of integration. Since a parabolic equation can be solved by a stepping procedure, this type of equation is particularly susceptible to numerical instability. It will be seen in the following pages that equivalent but different differential equations as well as a wide variety of finite difference approximations all may give rise to unstable numerical models. A stability analysis can predict which of these models is worthwhile to pursue. Two expressions for the VTE, a second order non-linear partial differential equation, will be considered. These are given by Equations (47) and (31) of the text and are repeated here for completeness. Remember that the interest in Equation (47) stems from the fact that it is a simplified form of Equation (3I). The two equations are, respectively,

$$
\frac{\partial \omega}{\partial t}+v_{z} a(1-\xi) \frac{\partial \omega}{\partial \xi}+v_{r}\left[b(1-n) \frac{\partial \omega}{\partial n}-\frac{\omega}{x}\right]=
$$




$$
\begin{gathered}
\frac{1}{R e y}\left\{a^{2}(1-\xi)^{2} \frac{\partial^{2} \omega}{\partial \xi^{2}}-a^{2}(1-\xi) \frac{\partial \omega}{\partial \xi}-\frac{\omega}{x^{2}}+\frac{b(1-n)}{x} \frac{\partial \omega}{\partial n}\right. \\
\left.+b^{2}(1-n)^{2} \frac{\partial^{2} \omega}{\partial n^{2}}-b^{2}(1-n) \frac{\partial \omega}{\partial \eta}\right\}
\end{gathered}
$$

and

$$
\begin{gathered}
\frac{\partial \omega}{\partial t}+a(1-\xi) \frac{\partial\left(v_{z} \omega\right)}{\partial \xi}+b(1-n) \frac{\partial\left(v_{r} \omega\right)}{\partial \eta}= \\
\frac{1}{R e y}\left\{a^{2}(1-\xi)^{2} \frac{\partial^{2} \omega}{\partial \xi^{2}}-a^{2}(1-\xi) \frac{\partial \omega}{\partial \xi}+\frac{b(1-n)}{x} \frac{\partial \omega}{\partial \eta}-\frac{\omega}{x^{2}}\right. \\
\left.+b^{2}(1-n)^{2} \frac{\partial^{2} \omega}{\partial n^{2}}-b^{2}(1-n) \frac{\partial \omega}{\partial \eta}\right\}
\end{gathered}
$$

A number of manipulations which have been proved capable of enhancing the stability of finite difference equations in the past will now be employed. It is the purpose here to introduce these ideas and to perhaps indicate how they may change stability. In this way, a rational approach is presented whereby one may learn to manipulate a difference equation, analyze its stability, and thereby derive a numerical model which is no more difficult than is necessary. In the interest of clarity, the derivations of the finite difference approximations to Equations $(D-1)$ and $(D-2)$ have been set aside in Appendix E. The analysis to be employed is that of von Neumann as it is discussed by O'Brien, et al. [33]. It will be described here using the nomenclature of the vorticity transport equation. It is assumed that 
for stability purposes the VTE may be linearized. This should not be too stringent since it may be argued that by being protected against small perturbations, the equation shouldn't experience any large perturbations which would render invalid the assumption of linearity. When $v_{z}$ and $v_{r}$ are slowly varying functions of time, it can be shown that the numerical error at the time $(n) \delta t$ and at the grid point $(i, j)$, i.e.,

$$
\varepsilon^{n}(i, j)=\omega^{n}(i, j)-\tilde{\omega}^{n}(i, j),
$$

satisfies the same difference equation as does the vorticity. In this expression,

$$
\begin{aligned}
& \omega^{n}(i, j) \text { is the numerical solution to the difference equation, } \\
& \text { and } \\
& \tilde{\omega}^{n}(i, j) \text { is the exact solution to the difference equation. }
\end{aligned}
$$

A Fourier decomposition of error is assumed of the form

$$
\varepsilon^{n}(i, j)=\varepsilon_{0} s^{n} e^{\hat{i}\left(k_{1} i+k_{2} j\right)}
$$

where

$$
\hat{i}^{2}=-1 \text {. }
$$

The wave number 


$$
k_{1}=\frac{2 \pi}{\lambda_{1}}
$$

refers to the variation of $\varepsilon^{n}(i, j)$ in the $\xi$-direction where $\lambda_{1}$ is the wave length measured in units of the index $i$. Similarly, $k_{2}$ is the wave number variation in the $n$-direction. The reference amplitude $\varepsilon_{0}$ is modified by $s^{\mathrm{n}}$ where $s$ is the growth rate factor and $\mathrm{n}$ is the index of time advancement. Note that if the component solution is to remain bounded as $\mathrm{n}$ becomes large, then

$$
|s| \leq 1
$$

The procedure is to write the finite difference equation in terms of the numerical error $\varepsilon^{n}(i, j)$ and its various permutations and subsequently to investigate what conditions must be met in terms of time step size, mesh size, and other parameters in order for the component solution to remain bounded as $\mathrm{n}$ becomes large.

On the following pages, the stability of four finite difference analogs to Equation (D-I) and one analog to Equation (D-2) will be investigated. Since the various approximations to Equation (D-I) differ only in the time derivative, the starting point for the first four cases is the finite difference equation

$$
\begin{aligned}
& \frac{\partial \omega}{\partial t}=-\left\{a v_{z}\left(\frac{M-i}{M}\right)\left[\frac{\omega(i+1, j)-\omega(i-1, j)}{2(1 / M)}\right]+\right. \\
& \left.v_{r}\left\langle b\left(\frac{M-i}{M}\right)\left[\frac{\omega(i, j+1)-\omega(i, j-1)}{2(1 / M)}\right]-\frac{\omega(i, j)}{x}\right\rangle\right\}
\end{aligned}
$$




$$
\begin{gathered}
+\frac{1}{R e y}\left\{a^{2}\left(\frac{M-i}{M}\right\}^{2}\left[\frac{\omega(i+1, j)+\omega(i-1, j)-2 \omega(i, j)}{(1 / M)^{2}}\right]-\right. \\
a^{2}\left(\frac{M-i}{M}\right)\left[\frac{\omega(i+1, j)-\omega(i-1, j)}{2(1 / M)}\right] \\
+\frac{b}{x}\left(\frac{M-j}{M}\right)\left[\frac{\omega(i, j+1)-\omega(i, j-1)}{2(1 / M)}\right]-\frac{\omega(i, j)}{x^{2}} \\
+b^{2}\left(\frac{M-j}{M}\right)^{2}\left[\frac{\omega(i, j+1)+\omega(i, j-1)-2 \omega(i, j)}{(1 / M)^{2}}\right]- \\
b^{2}\left(\frac{M-j}{M}\right)\left[\frac{\omega(i, j+1)-\omega(i, j-1)}{2(1 / M)}\right]
\end{gathered}
$$

This equation is derived in Appendix $E$ as Equation ( $E-2)$.

\section{Case I. Forward Time Difference--}

\section{Explicit Formulation of the Simplified VTE}

The simplest finite difference approximation to the time derivative is the forward difference which gives

$$
\frac{\partial \omega}{\partial t}=\frac{\omega^{n+1}(i, j)-\omega^{n}(i, j)}{\delta t}
$$

In this formulation, the $\omega(i, j)$ term on the right-hand side of Equation $(D-4)$ is evaluated at the time $(n) \delta t$. Combining the time derivative given above with Equation (D-4) and simplifying the resulting expression gives

$$
\frac{1}{\delta t} \omega^{n+1}(i, j)-\left\{\frac{1}{\delta t}-\frac{1}{R e y}\left[2 a^{2}(M-i)^{2}+2 b^{2}(M-j)^{2}+\frac{1}{x^{2}}-\frac{v_{r}^{n} \operatorname{Rey}}{x}\right]\right\} \omega^{n}(i, j)
$$




$$
\begin{aligned}
& =-\frac{a v_{z}^{n}(M-i)}{2}\left[\omega^{n}(i+1, j)-\omega^{n}(i-1, j)\right]- \\
& \quad \frac{b v_{r}^{n}(M-j)}{2}\left[\omega^{n}(i, j+1)-\omega^{n}(i, j-1)\right] \\
& +\frac{1}{\operatorname{Rey}\left\{a^{2}(M-i)^{2}\left[\omega^{n}(i+1, j)-\omega^{n}(i-1, j)\right]-\right.} \\
& \frac{a^{2}(M-i)}{2}\left[\omega^{n}(i+1, j)-\omega^{n}(i-1, j)\right] \\
& +b^{2}(M-j)^{2}\left[\omega^{n}(i, j+1)-\omega^{n}(i, j-1)\right]- \\
& \left.\frac{b^{2}(M-j)}{2}-\frac{b(M-j)}{2 x}\right]\left[\omega^{n}(i, j+1)-\omega^{n}(i, j-1)\right]
\end{aligned}
$$

Assuming that the velocities $v_{z}^{n}$ and $v_{n}^{n}$ are slowly varying functions of time so they may be written as

$$
\begin{array}{ll}
v_{z}^{n}=v_{z}^{n}(i, j)=v_{z 0}\left[1+\delta^{n}(i, j)\right] & \delta^{n}(i, j) \ll 1 \\
v_{r}^{n}=v_{r}^{n}(i, j)=v_{r 0}\left[1+\zeta^{n}(i, j)\right] & \zeta^{n}(i, j) \ll 1
\end{array}
$$

allows Equation $(D-5)$ to be linearized. Hence $v_{z}^{n}$ is replaced by $v_{z 0}$, and $v_{r}^{n}$ is replaced by $v_{r o}$. Since the numerical error

$$
\varepsilon^{n}(i, j)=\varepsilon_{0} s^{n} e^{\hat{i}\left(k_{1} i+k_{2} j\right)}
$$

satisfies the linearized form of Equation ( $D-5)$, this equation may now 
be rewritten in terms of the error as

$$
\begin{gathered}
\left\{\frac{1}{\delta t}\right\} \varepsilon^{n+1}(i, j)-\left\{\frac{1}{\delta t}-\frac{1}{R e y}\left[2 a^{2}(M-i)^{2}+\right.\right. \\
\left.\left.2 b^{2}(M-j)^{2}+\frac{1}{x^{2}}-\frac{v_{r 0} \operatorname{Rey}}{x}\right]\right\} \varepsilon^{n}(i, j) \\
=-\frac{a v_{z 0}(M-i)}{2}\left[\varepsilon^{n}(i+1, j)-\varepsilon^{n}(i-1, j)\right]-\frac{b v_{r 0}(M-j)}{2}\left[\varepsilon^{n}(i+1, j)-\varepsilon^{n}(i-1, j)\right] \\
+\frac{1}{\operatorname{Rey}\left\{a^{2}(M-i)^{2}\left[\varepsilon^{n}(i+1, j)+\varepsilon^{n}(i-1, j)\right]-\frac{a^{2}(M-i)}{2}\left[\varepsilon^{n}(i+1, j)-\varepsilon^{n}(i-1, j)\right]\right.} \\
\left.\left.+\frac{b^{2}(M-j)}{2}-\frac{b(M-j)}{2 x}\right]\left[\varepsilon^{n}(i, j+1)-\varepsilon^{n}(i, j-1)\right]\right\}
\end{gathered}
$$

Writing out the error term and noting that it may be simplified, for example

$$
\varepsilon^{n}(i+1, j)=\varepsilon_{0} s^{n} e^{\hat{i}\left(k_{1}[i+1]+k_{2} j\right)}=\left\{\varepsilon_{0} s^{n} e^{\hat{i}\left(k_{1} i+k_{2} j\right)}\right\} e^{\hat{i} k_{1}}
$$

allows cancellation of the grouping in the brackets. This is a coefficient of each term in Equation (D-6). The result of this simplification is

$$
\left\{\frac{1}{\delta t}\right\} s-\left\{\frac{1}{\delta t}-\frac{1}{\operatorname{Rey}}\left[2 a^{2}(M-i)^{2}+2 b^{2}(M-j)^{2}+\frac{1}{x^{2}}-\frac{v_{r 0} \operatorname{Rey}}{x}\right]\right\}
$$




$$
\begin{aligned}
= & -\frac{a v_{z 0}(M-i)}{2}\left[e^{-\hat{i} k_{1}}-e^{-\hat{i} k} 1\right]-\frac{b v_{r 0}(M-j)}{2}\left[e^{\hat{i} k}-e^{-\hat{i} k} 2\right] \\
& +\frac{1}{\operatorname{Rey}}\left\{a^{2}(M-i)^{2}\left[e^{\hat{i} k_{1}}+e^{-\hat{i} k} 1\right]-\frac{a^{2}(M-i)}{2}\left[e^{\hat{i} k}-e^{-\hat{i} k} 1\right]\right. \\
+ & \left.b^{2}(M-j)^{2}\left[e^{\hat{i} k}+e^{-\hat{i} k} 2\right]-\left[\frac{b^{2}(M-j)}{2}-\frac{b(M-j)}{2 x}\right]\left[e^{2}-e^{-\hat{i} k} 2\right]\right\}
\end{aligned}
$$

\section{Recognizing that}

$$
\sin x=\frac{e^{\hat{i} x}-e^{-\hat{i} x}}{2 \hat{i}} \text { and } \cos x=\frac{e^{\hat{i} x}+e^{-\hat{i} x}}{2}
$$

allows a further reduction to the form

$$
\begin{gathered}
\left\{\frac{1}{\delta t}\right\}_{s}-\left\{\frac{1}{\delta t}-\frac{1}{\operatorname{Rey}}\left[2 a^{2}(M-i)^{2}+2 b^{2}(M-j)^{2}+\frac{1}{x^{2}}-\frac{v_{r 0} \text { Rey }}{x}\right]\right\} \quad(D-7) \\
=-\left\{\left[a v_{z 0}(M-i)+\frac{a^{2}(M-i)}{R e y}\right] \sin k_{1}+\left[b v_{r o}(M-j)+\frac{b^{2}(M-j)}{R e y}\left(1-\frac{1}{b x}\right)\right] \sin k_{2}\right\} \hat{i} \\
+\frac{2}{R e y}\left\{a^{2}(M-i)^{2} \cos k_{1}+b^{2}(M-j)^{2} \cos k_{2}\right\}
\end{gathered}
$$

It is here assumed that $k_{1}=k_{2}=k$. This is a more stringent condition than unequal values of $k_{1}$ and $k_{2}$ since it allows both $k_{1}$ and $k_{2}$ to be maximized at the same time. Rearranging and simplifying Equation ( $D-7)$ gives the final form, namely,

$$
s=\alpha-\hat{i} \beta
$$


where

$$
\begin{aligned}
& \alpha=\frac{\delta t}{\operatorname{Rey}}\left\{\left[2 \mathrm{a}^{2}(M-i)^{2}+2 b^{2}(M-j)^{2}\right](\cos k-1)-\left[\frac{1}{x^{2}}-\frac{v_{r 0} \operatorname{Rey}}{x}\right]\right\}+1 \\
& \beta=\delta t\left\{a v_{z 0}(M-i)+b v_{r 0}(M-j)+\frac{a^{2}(M-i)}{R e y}+\frac{b^{2}(M-j)}{R e y}\left[1-\frac{1}{b x}\right]\right\} \sin k
\end{aligned}
$$

Since $s$ is complex, the condition on the growth rate factor for stability is that

$$
|s|^{2}=s \bar{s} \leq 1
$$

Because $s=\alpha-\hat{i} \beta$ and $\bar{s}=\alpha+\hat{i} \beta$, this becomes

$$
s \bar{s}=\alpha^{2}+\beta^{2} \leq 1
$$

Substituting the values of $\alpha$ and $\beta$ into this expression and going through some more tedious simplification yields the stability criterion

$$
\left(\frac{\delta t}{R e y}\right) \leq \frac{2 A}{A^{2}+B^{2}}
$$

where

$$
A=\left[2 a^{2}(M-i)^{2}+2 b^{2}(M-j)^{2}\right](1-\cos k)+\left[\frac{1}{x^{2}}-\frac{v_{r 0} \text { Rey }}{x}\right]
$$




$$
B=\left\{a(M-i)\left[v_{z 0} \operatorname{Rey}+a\right]+b(M-j)\left[v_{r 0} \operatorname{Rey}+b-\frac{1}{x^{2}}\right]\right\} \sin k
$$

Since by physical reasoning the quotient $\frac{\delta t}{R e y}$ must be positive, this requires that $A>0$. This inequality itself requires that

$$
\operatorname{Rey}<\frac{x}{v_{r 0}}\left\{\left[2 a^{2}(M-i)^{2}+2 b^{2}(M-j)^{2}\right](1-\cos k)+\frac{1}{x^{2}}\right\}
$$

Considering the worst possible case for which $\cos k=1$, the stability criterion becomes

$$
R e y<\frac{1}{x v_{\mathrm{ro}}}
$$

Since this finite difference equation is limited to small Reynolds numbers, it appears to be unacceptable.

\section{Case II. Central Time Difference--}

Explicit Formulation of the Simplified VTE

Dufort and Frankl [38] have postulated that a central time difference may improve the stability characteristics of a finite difference equation, and this opinion has been substantiated by Fromm [18]. The time derivative is approximated by

$$
\frac{\partial \omega}{\partial t}=\frac{\omega^{n+1}(i, j)-\omega^{n-1}(i, j)}{2(\delta t)}
$$

The analysis again begins with Equation (D-4) in which the $w(i, j)$ term on the right-hand side is evaluated at the time $(n) \delta t$. Since one 
investigation has already been conducted in a fairly detailed manner, the remaining ones will be covered in a few less steps. Combining Equations (D-9) and (D-4), linearizing the resulting equation, substituting in the Fourier decomposition of error, noting the trigonometric identities for $\sin x$ and $\cos x$, and simplifying yields the following expression for the growth rate factor $s$ :

$$
s^{2}-2(\alpha+\hat{i} \beta) s-1=0
$$

where

$$
\begin{aligned}
& \alpha=\frac{\delta t}{\operatorname{Rey}}\left\{\left[2 \mathrm{a}^{2}(M-i)^{2}+2 \mathrm{~b}^{2}(M-j)^{2}\right](\cos \mathrm{k}-1)-\frac{1}{x^{2}}+\frac{\mathrm{v}_{\mathrm{ro}} \operatorname{Rey}}{\mathrm{x}}\right\} \\
& \beta=-\frac{\delta t}{\operatorname{Rey}}\left\{\mathrm{a}(M-\mathrm{i})\left[\mathrm{v}_{\mathrm{z} 0} \operatorname{Rey}+\mathrm{a}\right]+\mathrm{b}(\mathrm{M}-\mathrm{j})\left[\mathrm{v}_{\mathrm{ro}_{0}} \operatorname{Rey}+\mathrm{b}-\frac{1}{\mathrm{x}}\right]\right\} \sin \mathrm{k}
\end{aligned}
$$

The assumption has been made that the wave numbers are equal, on

$$
k_{1}=k_{2}=k
$$

The quadratic equation for $s$ must now be solved to find under what conditions stability occurs. Stability is achieved when

$$
|s|^{2}=s \bar{s} \leq 1
$$

Since again $s$ is complex and since $s$ may be written as 


$$
\begin{aligned}
& s=x+\hat{i} y \\
& \bar{s}=x-\hat{i} y
\end{aligned}
$$

two simultaneous equations result from Equation (D-10). The first is found by equating the real coefficients to zero, and it shows that

$$
(x-\alpha)^{2}-(y-\beta)^{2}=1+\alpha^{2}-\beta^{2}
$$

The second is found by equating the imaginary coefficients to zero, and it reveals that

$$
1-\frac{\alpha}{x}-\frac{\beta}{y}=0
$$

Simultaneous solution of Equations (D-11) and (D-12) gives

$$
\begin{aligned}
& x_{1}=\alpha+\left\{\left[\frac{1+\alpha^{2}-\beta^{2}}{2}\right]+\left[\left(\frac{1+\alpha^{2}-\beta^{2}}{2}\right)+(\alpha \beta)^{2}\right]^{\frac{1}{2}}\right\}^{\frac{1}{2}} \\
& y_{1}=\frac{\beta x_{1}}{x_{1}-\alpha} \\
& x_{2}=\alpha-\left\{\left[\frac{1+\alpha^{2}-\beta^{2}}{2}\right]+\left[\left(\frac{1+\alpha^{2}-\beta^{2}}{2}\right)+(\alpha \beta)\right]^{\frac{1}{2}}\right\}^{\frac{1}{2}} \\
& y_{2}=\frac{\beta x_{2}}{x_{2}-\alpha}
\end{aligned}
$$

Two of the four possible solutions of the system of equations ( $D-11$ ) and (D-12) have been omitted because $\mathrm{x}$ and $\mathrm{y}$ were assumed real. 
Now for stability

$$
s \bar{s} \leq 1
$$

so

$$
\begin{aligned}
& s_{1} \bar{s}_{1}=\left(x_{1}+\hat{i}_{1}\right)\left(x_{1}-\hat{i}_{1}\right) \leq 1 \\
& s_{2} \bar{s}_{2}=\left(x_{2}+\hat{i}_{2}\right)\left(x_{2}-\hat{i}_{2}\right) \leq 1
\end{aligned}
$$

These expressions for $x_{1}, x_{2}, y_{1}$, and $y_{2}$ were investigated, and no values of $\alpha$ and $\beta$ were found for which both Equations (D-13) and (D-14) were satisfied. In fact, study of the limiting cases shows that

$$
|s|^{2} \leq 1 \text { only if } \alpha=0 \text { or } \quad \beta=0
$$

otherwise

$$
|s|^{2}>1
$$

which implies instability. Clearly, neither $\alpha$ nor $\beta$ can be guaranteed to vanish, so it appears that Case II is not an acceptable formulation either. However, if the equation for the error growth takes the form

$$
s^{2}-2(\alpha+\hat{i} \beta) s-\gamma=0
$$


where $\gamma<1$, then there does exist a region of stability. Hence, a new formulation must be found for which $\gamma<1$. The question now is, how can the finite difference equation be rearranged to allow $\gamma<1$ ?

Case III. Central Time Difference--

Explicit Formulation of the Simplified VTE

(Time Average Technique)

This particulan formulation is considered because it demonstrates how a troublesome term in a finite difference equation may be manipulated to enhance stability.

Once again, the analysis commences with Equation (D-4). In order to obtain an equation for the error growth in the form of Equation (D-15) for $\gamma<1$, the troublesome term of the right-hand side of Equation $(D-4)$ is rewritten as follows:

$$
\begin{gathered}
\frac{1}{\operatorname{Rey}}\left\{2 a^{2}(M-i)^{2}+2 b^{2}(M-j)^{2}+\frac{1}{x^{2}}-\frac{v_{r}^{n} \operatorname{Rey}}{x}\right\} \omega(i, j)= \\
\frac{1}{2 \operatorname{Rey}}\left\{2 a^{2}(M-i)^{2}+2 b^{2}(M-j)^{2}+\frac{1}{x^{2}}-\frac{v_{r}^{n} \operatorname{Rey}}{x}\right\} \\
{\left[\omega^{n+1}(i, j)+\omega^{n-1}(i, j)\right]}
\end{gathered}
$$

The central time difference is still used to approximate the time derivative, and it is

$$
\frac{\partial \omega}{\partial t}=\frac{\omega^{n+1}(i, j)-\omega^{n-1}(i, j)}{2 \delta t}
$$


The remaining vorticity terms are evaluated at the time ( $n) \delta t$. Incorporating these two approximations into Equation (D-4) and simplifying leads to

$$
\begin{aligned}
& \left\{\frac{1}{2 \delta t}+\frac{1}{2 \operatorname{Rey}}\left[2 a^{2}(M-i)^{2}+2 b^{2}(M-j)^{2}+\right.\right. \\
& \left.\left.\frac{1}{x^{2}}-\frac{v_{r}^{n+1} R e y}{x}\right]\right\} \omega^{n+1}(i, j) \\
& -\left\{\frac{1}{2 \delta t}-\frac{1}{2 \operatorname{Rey}}\left[2 a^{2}(M-i)^{2}+2 b^{2}(M-j)^{2}+\frac{1}{x^{2}}-\frac{v_{r}^{n-1} \operatorname{Rey}}{x}\right]\right\} \omega^{n-1}(i, j)= \\
& -\left\{\frac{v_{z}^{n} a(M-i)}{2}\left[\omega^{n}(i+1, j)-\omega^{n}(i-1, j)\right]+\right. \\
& \left.\frac{v_{r}^{n} b(M-j)}{2}\left[\omega^{n}(i, j+1)-\omega^{n}(i, j-1)\right]\right] \\
& +\frac{1}{\operatorname{Rey}}\left\{a^{2}(M-i)^{2}\left[\omega^{n}(i+1, j)+\omega^{n}(i-1, j)\right]-\right. \\
& \frac{a^{2}(M-1)}{2}\left[\omega^{n}(i+1, j)-\omega^{n}(i-1, j)\right] \\
& +b^{2}(M-j)^{2}\left[\omega^{n}(i, j+1)+\omega^{n}(i, j-1)\right]-\frac{b^{2}(M-j)}{2}\left[\omega^{n}(i, j+1)-\omega^{n}(i, j-1)\right] \\
& \left.+\frac{b(M-j)}{2 x}\left[\omega^{n}(i, j+1)-\omega^{n}(i, j-1)\right]\right\}
\end{aligned}
$$


is the term which must be reduced to a value less than one. Observation of Equation (D-16) reveals that the quotient of like coefficients is less than one for Rey small enough.

Linearizing Equation (D-16), substituting into it the Fourier decomposition of error, noting the trigonometric identities for $\sin \mathrm{x}$ and $\cos \mathrm{x}$, and reducing the resulting equation gives an expression for the growth rate factor $s$, namely,

$$
s^{2}-2(\alpha+\hat{i} \beta) s-\gamma=0
$$

where

$$
\begin{aligned}
& \alpha=\frac{\frac{1}{2}\left\{2 a^{2}(M-i)^{2}+2 b^{2}(M-j)^{2}\right\} \frac{\cos k}{\operatorname{Rey}}}{\frac{1}{2 \delta t}+\frac{1}{2 \operatorname{Rey}}\left\{2 a^{2}(M-i)^{2}+2 b^{2}(M-j)^{2}+\frac{1}{x^{2}}-\frac{v_{r o} \operatorname{Rey}}{x}\right\}} \\
& \beta=\frac{-\frac{1}{2}\left\{a(M-i)\left[v_{z 0}+\frac{a}{\operatorname{Rey}}\right]+b(M-j)\left[v_{r o}+\frac{b}{\operatorname{Rey}}-\frac{1}{x \operatorname{Rey}}\right]\right\} \sin k}{\frac{1}{2 \delta t}+\frac{1}{2 \operatorname{Rey}}\left\{2 a^{2}(M-i)^{2}+2 b^{2}(M-j)^{2}+\frac{1}{x^{2}}-\frac{v_{r o} \operatorname{Rey}}{x}\right\}} \\
& \gamma=\frac{\frac{1}{2 \delta t}-\frac{1}{2 \operatorname{Rey}}\left\{2 a^{2}(M-i)^{2}+2 b^{2}(M-j)^{2}+\frac{1}{x^{2}}-\frac{v_{r o} \text { Rey }}{x}\right\}}{\frac{1}{2 \delta t}+\frac{1}{2 \operatorname{Rey}}\left\{2 a^{2}(M-1)^{2}+2 b^{2}(M-j)^{2}+\frac{1}{x^{2}}-\frac{v_{r 0} \text { Rey }}{x}\right\}}
\end{aligned}
$$

The method of solution of Equation (D-17) follows exactly the procedure used in solving Equation (D-10) except that the parameter $\gamma$ appears in 
place of the "l" in Equation (D-10). The growth rate factor is assumed to be of the form

$$
s=x+\hat{i} y
$$

Consequently, the solution of the simultaneous equations resulting from Equation $(D-17)$ is

$x_{1}=\alpha+\left\{\left(\frac{\gamma+\alpha^{2}-\beta^{2}}{2}\right)+\left[\left(\frac{\gamma+\alpha^{2}-\beta^{2}}{2}\right)^{2}+(\alpha \beta)^{2}\right]^{\frac{1}{2}}\right\}^{\frac{1}{2}} \quad y_{1}=\frac{\beta x_{1}}{x_{1}-\alpha}$

$x_{2}=\alpha-\left\{\left(\frac{\gamma+\alpha^{2}-\beta^{2}}{2}\right)+\left[\left(\frac{\gamma+\alpha^{2}-\beta^{2}}{2}\right)^{2}+(\alpha \beta)^{2}\right]^{\frac{1}{2}}\right\}^{\frac{1}{2}} \quad y_{2}=\frac{\beta x_{2}}{x_{2}-\alpha}$

Again, two solutions have been neglected since it was assumed that both $\mathrm{x}$ and $\mathrm{y}$ were real. Since $\mathrm{y}$ is less than unity for Rey small enough, a region of stability may be found. This is given by

$$
s \bar{s} \leq 1 \quad \text { if } \quad\left\{\begin{array}{c}
-0.1 \leq \alpha \leq 0.1 \\
-0.6 \leq \beta \leq 0.6 \\
\gamma \leq 0.7
\end{array}\right.
$$

This conclusion was reached simply by substituting values of $\alpha, \beta$, and $\gamma$ into the expressions for $x_{1}, y_{1}, x_{2}$, and $y_{2}$. Both values of $x$ and both values of $y$ must satisfy the condition that 


$$
s \bar{s} \leq 1
$$

This straightforward numerical calculation demonstrates the simplicity and utility of Equation (D-17). This is an important point. Once the growth rate factor is expressed in the form of Equation (D-17), the conditions for stability are given by Equations (D-21) through (D-23). Now the expressions for $\alpha, \beta$, and $\gamma$ may be investigated to determine the limitations on Rey, $\delta t$, and the other parameters which are necessary for stability. Consider first the condition that $\alpha<0.1$. From Equation (D-18), this requires that

$$
\frac{\left[2 a^{2}(M-i)^{2}+2 b^{2}(M-j)^{2}+\frac{1}{x^{2}}-\frac{v_{r 0} \operatorname{Rey}}{x}\right]-\frac{1}{x^{2}}+\frac{v_{r} \operatorname{Rey}}{x}}{\frac{R e y}{\delta t}+\left[2 a^{2}(M-i)^{2}+2 b^{2}(M-j)^{2}+\frac{1}{x^{2}}-\frac{v_{r 0} \operatorname{Rey}}{x}\right]} \cos k<0.1
$$

Simplifying this expression and choosing $k$ so that the left-hand side of this inequality is a maximum, i.e.

$$
\cos k=1
$$

yields

$$
\begin{aligned}
& \frac{R e y}{\delta t}>\left(\frac{1}{0.1}-1\right) {\left[2 a^{2}(M-i)^{2}+2 b^{2}(M-j)^{2}+\frac{1}{x^{2}}-\frac{v_{\text {ro }} \text { Rey }}{x}\right] } \\
&+\frac{1}{0.1}\left(\frac{v_{\text {ro }} \text { Rey }}{x}-\frac{1}{x^{2}}\right)
\end{aligned}
$$


which simplifies to

$$
\frac{R e y}{\delta t}>9\left[2 a^{2}(M-i)^{2}+2 b^{2}(M-j)^{2}+\frac{1}{x^{2}}-\frac{v_{r 0} \text { Rey }}{x}\right]+\frac{1}{10}\left(\frac{v_{r_{0}} \text { Rey }}{x}-\frac{1}{x^{2}}\right)(D-24)
$$

Note that consideration of the other equality,

$$
\alpha>-0.1
$$

gives the same expression as Equation (D-24). Equation (D-24) will be left for the moment to see what the other inequalities require. There's always the chance that some other condition on the stability will make the evaluation of Equation (D-21) unnecessary.

Now investigate the condition on $\gamma$ which states that

$$
\gamma \leq 0.7
$$

Combining Equations $(D-25)$ and $(D-20)$ and simplifying allows the derivation of

$$
\frac{\operatorname{Rey}}{\delta t} \leq\left(\frac{1+0.7}{1-0.7}\right)\left[2 a^{2}(M-i)^{2}+26^{2}(M-j)^{2}+\frac{1}{x^{2}}-\frac{v_{r_{0}} \text { Rey }}{x}\right]
$$

which simplifies to

$$
\frac{\operatorname{Rey}}{\delta t}<6\left[2 \mathrm{a}^{2}(\mathrm{M}-i)^{2}+2 \mathrm{~b}^{2}(M-j)^{2}+\frac{1}{\mathrm{x}^{2}}-\frac{\mathrm{v}_{\mathrm{ro}} \text { Rey }}{\mathrm{x}}\right]
$$


The combination of Equations (D-24) and (D-26) should now yield a simple but very useful stability criterion. Combining these two expressions and reducing the result finally yields the inequality

$$
\frac{R e y}{\delta t}<\frac{3}{x}\left[\frac{\overline{1}}{x}-v_{\text {no }} \operatorname{Rey}\right]
$$

For any physically reasonable problem

$$
\frac{R e y}{\delta t}>0
$$

and combining this fact with Equation (D-27) requires at the very least that

$$
\text { Rey }<\frac{1}{\mathrm{v}_{\text {ro }} \mathrm{X}}
$$

Thus it appears that the formulation studied in this case is Iimited to small Reynolds numbers. This is insufficient for the purposes of this study.

However, several points of very practical importance have been demonstrated. Recall that no conditions for stability could be found for the finite difference approximation of Case II. To obtain the difference equation of Case III, a simple "trick," a time average, was employed. The resulting equation is stable although only for small Reynolds numbers. Finally and most important, it is demonstrated in this particular stability analysis that if the complex quadratic equa- 
tion for the growth rate factor can be written in the form of Equation (D-17), the conditions for stability are given by Equations (D-21), $(D-22)$, and $(D-23)$. The limitations on the parameters $\alpha, \beta$, and $\gamma$ can now be found quite easily in terms of the Reynolds number, time increment, mesh spacing, and other parameters of the problem.

\section{Case IV. Forward Time Difference--}

Implicit Formulation of the Simplified VTE

The analysis stems from Equation (D-4), and the time derivative is written as a forward difference, namely

$$
\frac{\partial \omega}{\partial t}=\frac{\omega^{n+1}(i, j)-\omega^{n}(i, j)}{\delta t}
$$

In the light of Equation (D-28), it is clearly more accurate to evaluate all terms on the right-hand side of Equation (D-4) at the midpoint of the time interval bounded by this expression, i.e., at $t=\left(n+\frac{1}{2}\right) \delta t$. Consequently, Equation (D-28) now becomes a time average.

Since this stability analysis is slightly different from the preceding ones, it will be developed in a little more detail. The vorticity $\omega(i, j)$ is evaluated at the mean time $\left(n+\frac{1}{2}\right) \delta t$ using the time average. Hence, for example,

$$
\omega(i, j)=\omega^{n+1 / 2}(i, j)=\frac{1}{2}\left[\omega^{n+1}(i, j)+\omega^{n}(i, j)\right]
$$

Applying this expression and the forward time difference to Equation $(\mathrm{D}-4)$, and simplifying gives 


$$
\begin{aligned}
& \left\{\frac{1}{\delta t}+\frac{1}{2 \operatorname{Rey}}\left[2 a^{2}(M-i)^{2}+2 b^{2}(M-j)^{2}+\frac{1}{x^{2}}-\frac{v_{r}^{n+1} \operatorname{Rey}}{x}\right]\right\} \omega^{n+1}(i, j) \quad(D-29) \\
& -\left\{\frac{1}{\delta t}-\frac{1}{2 \operatorname{Rey}}\left[2 a^{2}(M-i)^{2}+2 b^{2}(M-j)^{2}+\frac{1}{x^{2}}-\frac{v_{r}^{n} \operatorname{Rey}}{x}\right]\right\} \omega^{n}(i, j)= \\
& -\left\{\frac{a v_{z}(M-i)}{2}[\omega(i+1, j)-\omega(i-1, j)]+\frac{b v_{r}(M-j)}{2}[\omega(i, j+1)-\omega(i, j-1)]\right\} \\
& +\frac{1}{\operatorname{Rey}}\left\{a^{2}(M-i)^{2}[\omega(i+1, j)+\omega(i-1, j)]-\frac{a^{2}(M-i)}{2}[\omega(i+1, j)-\omega(i-1, j)]\right. \\
& +b^{2}(M-j)^{2}[\omega(i, j+1)+\omega(i, j-1)]-\frac{b^{2}(M-j)}{2}[\omega(i, j+1)-\omega(i, j-1)] \\
& \left.+\frac{b(M-j)}{2 x}[\omega(i, j+1)-\omega(i, j-1)]\right\}
\end{aligned}
$$

where all the terms on the right-hand side must be evaluated at the time $\left(n+\frac{1}{2}\right) \delta t$. Again, the time average is used, and a sample term is given by

$$
\omega^{n+1 / 2}(i+1, j)=\frac{1}{2}\left\{\omega^{n+1}(i+1, j)+\omega^{n}(i+1, j)\right\}
$$

Equation (D-29) is linearized so that the error term $\varepsilon(i, j)$ satisfies the same finite difference equation. Note here that since every vorticity term is written as a time average, the resulting expression for the error has a coefficient of $(s+1)$. For example, 


$$
\begin{aligned}
\varepsilon^{n+1 / 2}(i, j) & =1 / 2\left\{\varepsilon^{n+1}(i, j)+\varepsilon^{n}(i, j)\right\} \\
& =1 / 2\left\{\varepsilon_{0} s^{n+1} e^{\hat{i}\left(k_{1} i+k_{2} j\right)}+\varepsilon_{0} s^{n} e^{\hat{i}\left(k_{1} i+k_{2} j\right)}\right\} \\
& =1 / 2(s+1) \varepsilon^{n}(i, j)
\end{aligned}
$$

Hence, the expression for the error growth which stems from Equation (D-29) can almost be written by inspection. Substituting the error term into the linearized form of Equation (D-29), simplifying, noting the trigonometric identities for $\sin x$ and $\cos x$, and assuming equal wave numbers $k_{1}$ and $k_{2}$ gives finally

$$
\begin{gathered}
\left\{\frac{1}{\delta t}+\frac{1}{2 \operatorname{Rey}}\left[2 a^{2}(M-i)^{2}+2 b^{2}(M-j)^{2}+\frac{1}{x^{2}}-\frac{v_{r 0} \operatorname{Rey}}{x}\right]\right\} s \quad(D-30) \\
-\left\{\frac{1}{\delta t}-\frac{1}{2 \operatorname{Rey}}\left[2 a^{2}(M-i)^{2}+2 b^{2}(M-j)^{2}+\frac{1}{x^{2}}-\frac{v_{r 0} \operatorname{Rey}}{x}\right]\right\}= \\
-\left\{\frac{a(M-i)}{2}\left[v_{z 0}+\frac{a}{\operatorname{Rey}}\right]+\frac{b(M-j)}{2}\left[v_{r 0}+\frac{b}{\operatorname{Rey}}-\frac{1}{x \operatorname{Rey}}\right]\right\} \hat{i} \sin k(s+1) \\
+\frac{1}{\operatorname{Rey}}\left\{a^{2}(M-i)^{2}+b^{2}(M-j)^{2}\right\} \cos k(s+1)
\end{gathered}
$$

Dividing through by the coefficient of the first term of this equation and reducing the result gives

$$
s-\gamma=(\alpha-\hat{i} \beta)(s+1)
$$


where here

$$
\begin{aligned}
& \alpha=\frac{\left[a^{2}(M-i)^{2}+b^{2}(M-j)^{2}\right] \cos k}{\frac{\operatorname{Rey}}{\delta t}+\frac{1}{2}\left\{2 a^{2}(M-i)^{2}+2 b^{2}(M-j)^{2}+\frac{1}{x^{2}}-\frac{v_{r 0} \operatorname{Rey}}{x}\right\}} \quad \text { (D-32) } \\
& B=\frac{\operatorname{Rey}\left\{a(M-i)\left[v_{z 0}+\frac{a}{\operatorname{Rey}}\right]+b(M-j)\left[v_{r 0}+\frac{b}{\operatorname{Rey}}-\frac{1}{x \text { Rey }}\right] \cdot \sin k\right.}{\frac{\operatorname{Rey}}{\delta t}+\frac{1}{2}\left\{2 a^{2}(M-i)^{2}+2 b^{2}(M-j)^{2}+\frac{1}{x^{2}}-\frac{v_{r 0} \operatorname{Rey}}{x}\right\}} \text { (D-33) } \\
& \gamma=\frac{\left.\frac{\operatorname{Rey}}{\delta t}-\frac{1}{2} i 2 a^{2}(M-i)^{2}+2 b^{2}(M-j)^{2}+\frac{1}{x^{2}}-\frac{v_{r 0} \operatorname{Rey}}{x}\right\}}{\frac{\operatorname{Rey}}{\delta t}+\frac{1}{2}\left(2 a^{2}(M-i)^{2}+2 b^{2}(M-j)^{2}+\frac{1}{x^{2}}-\frac{v_{r 0} \operatorname{Rey}}{x}\right\}} \quad \text { (D-34) }
\end{aligned}
$$

Solving Equation (D-3I) for the growth rate factor gives

$$
s=\frac{(\gamma+\alpha)-\hat{i} \beta}{(1-\alpha)+\hat{i} \beta}
$$

Since $s$ is complex, the requirement for stability is

$$
s \bar{s}=\frac{(\gamma+\alpha)^{2}+\beta^{2}}{(1-\alpha)^{2}+\beta^{2}} \leq 1
$$

Expanding and simplifying this yields

$$
2 \alpha(1+\gamma) \leq 1-\gamma^{2}
$$

In order to continue, an assumption must be made about the sign of the term $(1+\gamma)$. Assume for the moment that 


$$
(1+\gamma)>0
$$

and see where it leads. Equation (D-36) now dictates that for stability

$$
\alpha \leq \frac{1}{2}(1-\gamma)
$$

Substituting in the values of $\alpha$ and $\gamma$ given respectively by Equations $(D-32)$ and $(D-34)$ presents the inequality

$$
\frac{\left[a^{2}(M-i)^{2}+b^{2}(M-j)^{2}\right] \cos k}{\frac{R e y}{\delta t}+F F} \leq \frac{1}{2}\left\{\frac{\frac{R e y}{\delta t}+F F}{\frac{R e y}{\delta t}+F F}-\frac{\frac{R e y}{\delta t}-F F}{\frac{R e y}{\delta t}+F F}\right\}
$$

where FF is simply a shorthand notation for the term

$$
F F=\frac{1}{2}\left\{2 a^{2}(M-i)^{2}+2 b^{2}(M-j)^{2}+\frac{1}{x^{2}}-\frac{v_{r 0} \operatorname{Rey}}{x}\right\}
$$

In order to evaluate the inequality expressed by Equation ( $D-38)$, it is now assumed that

$$
\frac{R e y}{\delta t}+F F>0
$$

Application of this expression allows elimination of the divisor of Equation (D-38), and further simplification and rearrangement gives finally the condition for stability 


$$
\frac{1}{2}\left[\frac{v_{r 0} \text { Rey }}{x}-\frac{1}{x^{2}}\right] \leq\left[a^{2}(M-i)^{2}+b^{2}(M-j)^{2}\right](1-\cos k)
$$

The right-hand side of this expression is always positive, although in the worst possible case it may approach zero. In this circumstance, the Reynolds number on the left-hand side must be tiny indeed. Thus it seems that the same limitation, small Reynolds number, which has plagued these analyses from the start has arisen again. For this reason, the formulation may have to be abandoned. This is disappointing, because an implicit scheme has been shown in the past to have good stability characteristics. However, remember that the limitation of this formulation is not on the size of the time step but on the range of physical problems which can be solved. This range is determined by the Reynolds number.

Several assumptions were made in the development of Equation $(D-40)$. If these are invalid, other assumptions must be made to take their place, and a new analysis must be performed.

Consider first the assumption of Equation (D-39). Rearrangement of this inequality shows that

$$
\operatorname{Rey}\left\{\frac{1}{2} \frac{v_{r 0}}{x}-\frac{1}{\delta t}\right\}<\frac{1}{2}\left\{2 a^{2}(M-i)^{2}+2 b^{2}(M-j)^{2}+\frac{1}{x^{2}}\right\}
$$

Here, the right-hand side is always positive, and the left-hand side dictates again a small Reynolds number if its coefficient is positive. However, the left-hand side is negative for a small enough time increment. Neediess to say, a small time increment is not too desirable. 
Apparently then, Equation (D-39) is a valid assumption.

Now, investigate the inequality expressed by Equation (D-37).

Substituting the value of $\gamma$ from Equation (D-34) into Equation (D-37)

and simplifying reveals that

$$
\frac{\frac{R e y}{\delta t}}{\frac{R e y}{\delta t}+\frac{1}{2}\left\{2 a^{2}(M-i)^{2}+2 b^{2}(M-j)^{2}+\frac{1}{x^{2}}-\frac{v_{r 0} \text { Rey }}{x}\right\}}>0
$$

For a physically reasonable problem, the numerator of this expression must be positive, and the denominator is positive for small enough Rey. Hence, the validity of Equation (D-37) is established, and it is shown that Equations (D-39) and (D-37) are compatible.

Just to make sure nothing has been missed in this implicit finite difference formulation of the VTE, it is interesting to note the effect on Equation (D-36) of the assumptions.

(1) $\frac{\text { Rey }}{\delta t}+\frac{1}{2}\left\{2 a^{2}(M-i)^{2}+2 b^{2}(M-j)^{2}+\frac{1}{x^{2}}-\frac{v_{r 0} \text { Rey }}{x}\right\}<0$ (D-4I)

(2) $1+\gamma<0$

These are the only other assumptions which can be made which are compatible with each other. Investigation of Equations (D-4I) and (D-42) reveals that both inequalities can be satisfied only if 


$$
\frac{R e y}{\delta t}<0
$$

Clearly, this is not physically possible.

The conclusion to be reached at this point is that all of the finite difference approximations introduced to this point in the analysis are limited to small Reynolds numbers. This is especially disappointing in Case IV, because in the past, implicit formulations have usually produced remarkable properties of stability. This is shown by Pearson [32]. The question to be asked now is, what can be learned from the analysis of Case IV which will indicate a new approach?

Consider once again the inequality which limits the finite difference formulation to small Reynolds numbers, namely,

$$
\frac{R e y}{\delta t}+\left\{a^{2}(M-i)^{2}+b^{2}(M-j)^{2}+\frac{1}{2 x}\left(\frac{1}{x}-v_{r o} R e y\right)\right\}>0
$$

which itself implies that

$$
1+\gamma>0
$$

The troublesome quantity is $\mathrm{v}_{\text {ro }}$ Rey preceded by a minus sign. This term is a direct result of the simplification of the VTE effected by the use of the continuity equation. It is important to note that in the two-dimensional continuity equation, the term does not arise. Hence, the two-dimensional analog to Equation (47) of the text should 
not experience a limitation on stability by a small Reynolds number. The stability difficulty arising from employing the continuity equation in deriving the VTE is a three-dimensional phenomenon alone. This contention has been verified by studying the stability of the twodimensional VTE.

The investigation of the stability of the VTE will now be pursued by returning to the unsimplified VTE of Equation (31).

\section{Case V. Forward Time Difference--}

Implicit Formulation of the Unsimplified VTE

For this case the governing partial differential equation is specified by Equation (3I). The forward time difference is employed so that

$$
\frac{\partial \omega}{\partial t}=\frac{\omega^{n+1}(i, j)-\omega^{n}(i, j)}{\delta t}
$$

The finite difference expression for Equation (31) is derived as Equation ( $E-21$ ) in Appendix $E$, and an unsimplified form is given as

$$
\begin{gathered}
\frac{\omega^{n+1}(i, j)-\omega^{n}(i, j)}{\delta t}= \\
-\frac{a(M-i)}{2}\left[v_{z}(i+1, j) \omega(i+1, j)-v_{z}(i-1, j) \omega(i-1, j)\right] \\
-\frac{b(M-j)}{2}\left[v_{r}(i, j+1) \omega(i, j+1)-v_{r}(i, j-1) \omega(i, j-1)\right]
\end{gathered}
$$




$$
\begin{aligned}
& +\frac{1}{\operatorname{Rey}}\left\{a^{2}(M-i)^{2}[\omega(i+1, j)+\omega(i-1, j)]-\frac{a^{2}(M-i)}{2}[\omega(i+1, j)-\omega(i-1, j)]\right. \\
& +b^{2}(M-j)^{2}[\omega(i, j+1)+\omega(i, j-1)]-\frac{b^{2}(M-j)}{2}[\omega(i, j+1)-\omega(i, j-1)] \\
& \left.+\frac{b(M-j)}{2 x}[\omega(i, j+1)-\omega(i, j-1)]\right\} \\
& -\frac{1}{\operatorname{Rey}}\left[2 a^{2}(M-i)^{2}+2 b^{2}(M-j)^{2}+\frac{1}{x^{2}}\right] \omega(i, j)
\end{aligned}
$$

For an implicit formulation, all the terms on the right-hand side of Equation (D-43) must be evaluated at the time $\left(n+\frac{1}{2}\right) \delta t$. Again, the time average is employed, e.g.

$$
\omega^{n+1 / 2}(i, j)=\frac{1}{2}\left\{\omega^{n+1}(i, j)+\omega^{n}(i, j)\right\}
$$

Using this time average for the term $\omega(i, j)$ in Equation $(D-43)$ and simplifying yields

$$
\begin{aligned}
& \left\{\frac{1}{\delta t}+\frac{1}{\operatorname{Rey}}\left[a^{2}(M-i)^{2}+b^{2}(M-j)^{2}+\frac{1}{2 x^{2}}\right]\right\} \omega^{n+1}(i, j) \\
& -\left\{\frac{1}{\delta t}-\frac{1}{\operatorname{Rey}}\left[a^{2}(M-i)^{2}+b^{2}(M-j)^{2}+\frac{1}{2 x^{2}}\right]\right\} \omega^{n}(i, j)= \\
& -\frac{a(M-i)}{2}\left[v_{z}(i+1, j) \omega(i+1, j)-v_{z}(i-1, j) \omega(i-1, j)\right]
\end{aligned}
$$




$$
\begin{gathered}
-\frac{b(M-j)}{2}\left[v_{r}(i, j+1) \omega(i, j+1)-v_{r}(i, j-1) \omega(i, j-1)\right] \\
+\frac{1}{R e y}\left\{a^{2}(M-i)^{2}[\omega(i+1, j)+\omega(i-1, j)]-\frac{a^{2}(M-i)}{2}[\omega(i+1, j)-\omega(i-1, j)\right. \\
\left.+b^{2}(M-j)^{2}[\omega(i, j+1)+\omega(i, j-1)]-\frac{b^{2}(M-j)}{2}\left(1-\frac{1}{b x}\right)[\omega(i, j+1)-\omega(i, j-1)]\right\}
\end{gathered}
$$

This equation is now linearized, and following a similar analysis of Fromm [18], all second-order terms in the velocity perturbations are neglected. Substituting the numerical error

$$
\varepsilon^{n}(i, j)=\varepsilon_{0} s^{n} e^{\hat{i}\left(k_{1} i+k_{2} j\right)}
$$

into the resulting equation, noting the trigonometric identities for sin $x$ and $\cos x$, and simplifying gives the expression

$$
\begin{aligned}
& \left\{\frac{1}{\delta t}+\frac{1}{\operatorname{Rey}}\left[a^{2}(M-i)^{2}+b^{2}(M-j)^{2}+\frac{1}{2 x^{2}}\right]\right\} s \\
& -\left\{\frac{1}{\delta t}-\frac{1}{\operatorname{Rey}}\left[a^{2}(M-i)^{2}+b^{2}(M-j)^{2}+\frac{1}{2 x^{2}}\right]\right\}= \\
& \frac{1}{\operatorname{Rey}}\left\{a^{2}(M-i)^{2}+b^{2}(M-j)^{2}\right\} \cos k(s+1)
\end{aligned}
$$


$-\frac{1}{\operatorname{Rey}}\left\{\frac{\mathrm{a}(\mathrm{M}-\mathrm{i})}{2}\left[\mathrm{v}_{\mathrm{z}_{0}} \operatorname{Rey}+\mathrm{a}\right]+\frac{\mathrm{b}(\mathrm{M}-\mathrm{j})}{2}\left[\mathrm{v}_{\mathrm{r}_{0}} \operatorname{Rey}+\mathrm{b}-\frac{1}{x}\right]\right\} \hat{i} \sin \mathrm{k}(s+1)$

Only the parameter $k$ appears in this equation because it has already been assumed that the wave numbers are all equal, i.e.,

$$
k_{1}=k_{2}=k
$$

Multiplying Equation (D-45) through by the non-zero Reynolds number, and reducing the result gives finally the expression for the growth rate factor $s$,

$$
s-\gamma=(\alpha-\hat{i} \beta)(s+1)
$$

where

$$
\begin{gathered}
\alpha=\frac{\left[a^{2}(M-i)^{2}+b^{2}(M-j)^{2}\right] \cos k}{\frac{R e y}{\delta t}+\left[a^{2}(M-i)^{2}+b^{2}(M-j)^{2}+\frac{1}{2 x^{2}}\right]} \\
\beta=\frac{\left[\frac{a(M-i)}{2}\left(v_{z 0} \operatorname{Rey}+a\right)+\frac{b(M-j)}{2}\left(v_{r o} \operatorname{Rey}+b-\frac{1}{x}\right)\right] \sin k}{\frac{R e y}{\delta t}+\left[a^{2}(M-i)^{2}+b^{2}(M-j)^{2}+\frac{1}{2 x^{2}}\right]}
\end{gathered}
$$




$$
\gamma=\frac{\frac{R e y}{\delta t}-\left[a^{2}(M-i)^{2}+b^{2}(M-j)^{2}+\frac{1}{2 x^{2}}\right]}{\frac{R e y}{\delta t}+\left[a^{2}(M-i)^{2}+b^{2}(M-j)^{2}+\frac{1}{2 x^{2}}\right]}
$$

Equation (D-47) has the same form as Equation (D-31). Solving for $s$ in Equation (D-47) gives the growth rate factor as

$$
s=\frac{(\gamma+\alpha)-\hat{i} \beta}{(1-\alpha)+\hat{i} \beta}
$$

Since $s$ is complex, the condition for stability is

$$
s \bar{s}=\frac{(\gamma+\alpha)^{2}+\beta^{2}}{(1-\alpha)^{2}+\beta^{2}} \leq 1
$$

This leads to the stability criterion

$$
2 \alpha(1+\gamma) \leq 1-\gamma^{2}
$$

To investigate this expression, two simplifying assumptions are made. These are:

(1) $1+\gamma>0$

(2) $\frac{R e y}{\delta t}+\left[a^{2}(M-i)^{2}+b^{2}(M-j)^{2}+\frac{1}{2 x^{2}}\right]>0$ 
Under these assumptions, Equation (D-5I) reduces to the stability criterion

$$
\left[a^{2}(M-i)^{2}+b^{2}(M-j)^{2}\right](1-\cos k)+\frac{1}{2 x^{2}} \geq 0
$$

This inequality is always satisfied. The conditions on the unknown wave number in the term $(1-\cos k)$ need not even be investigated. Now consider the effect of the assumptions. For

$$
\frac{\text { Rey }}{\delta t}>0
$$

Equation (D-53) is always satisfied. Substituting Equation (D-50) into Equation (D-52) and simplifying gives

$$
\frac{2 \frac{R e y}{\delta t}}{\frac{R e y}{\delta t}+\left[a^{2}(M-i)^{2}+b^{2}(M-j)^{2}+\frac{1}{2 x^{2}}\right]}>0
$$

This expression too is always satisfied for

$$
\frac{R e y}{\delta t}>0
$$

Hence, it appears that the finite difference expression for Equation (31) of the text is always stable when it is written implicitly using central differences. 
of course, other assumptions can be made to simplify Equation (D-5I). However, the only two compatible ones, namely that

$$
\begin{gathered}
1+y<0 \\
\frac{R e y}{\delta t}+\left[a^{2}(M-i)^{2}+b^{2}(M-j)^{2}+\frac{1}{2 x^{2}}\right]<0
\end{gathered}
$$

lead to the inequality that

$$
\frac{R e y}{\delta t}<0
$$

This is not physically reasonable.

\section{Conclusions}

The most important result this Appendix offers is the conclusion that an implicit formulation of Equation (31) is stable with a very general stability criterion, namely

$$
\frac{R e y}{\delta t}>0
$$

The actual derivation of the finite difference equation is given in Case V, Appendix E.

A recommendation may also be made at this point concerning the conduction of a stability analysis. Although the result of an analysis of a particular equation is unsatisfactory and gives an unacceptable 
stability criterion, the investigation itself should be studied carefully to obtain clues which will be beneficial in postulating a new finite difference approximation. This was the procedure which was applied in this Appendix. Cases I and II are applications of standard time derivatives used in the formulation of time dependent finite difference equations. Neither of the equations is necessarily unstable, but both are definitely limited to small Reynolds numbers. This is an undesirable constraint. However, the stability analysis of Case II reveals that a very slight change in the difference equation leads to a new stable expression. In fact, it is shown that if the expression for the growth rate factor can be written in the form of the complex, quadratic Equation (D-17), the stability of an equation may be studied quite easily just by investigating a number of coefficients exemplified by those of Equations (D-18), (D-19), and (D-20). However, once again, the finite difference equation is limited to small Reynolds numbers. Remember that these first three equations are explicit, and their importance lies in their simplicity.

Case IV is an implicit formulation of the partial differential equation, Equation (47). Usually, implicit difference equations exhibit better stability characteristics than do their explicit counterparts. This equation too is limited to small Reynolds numbers, but study of the results reveals the term which is responsible for the difficulty. It can be seen that the Reynolds number limitation is due to the three-dimensionality of the problem. This is an important finding, because similar problems may arise in other three-dimensional equations. Identification of the troublesome term of Case IV shows the way to a new 
formulation, that of Case $\mathrm{V}$, which is stable for all Reynolds numbers. It is relatively unimportant that the analyses of the first four cases is based upon a poor formulation of the original differential equation, but it is important to recognize the logic of the sequence of events which finally lead to Case V.

Finally, it should be mentioned that the limitation on stability of low Reynolds numbers for some of these formulations is based on the unknown values of the parameters $v_{z_{0}}$ and $x$. After a problem has been solved, these terms may be evaluated, and it can be determined whether or not there ever existed a real threat to stability. However, when nothing of the solution is known a priori, a finite difference equation which has been analyzed as stable must be used. 
APPENDIX E

FINITE DIFFERENCE FORMS OF THE VORTICITY TRANSPORT EQUATION

The VTE is developed in the body of this research in two different forms. They are the simplified VTE given by Equation (47) and the unsimplified VTE given by Equation (31). Since the simplified VTE is the basis for the stability analyses of the first four cases of Appendix D, the various finite difference formulations to Equation (47) are investigated first.

\section{The Simplified VTE}

Equation (47) is repeated here as

$$
\begin{gathered}
\frac{\partial \omega}{\partial t}+v_{z} a(1-\xi) \frac{\partial \omega}{\partial \xi}+v_{r}\left[b(1-n) \frac{\partial \omega}{\partial n}-\frac{\omega}{x}\right]= \\
\frac{1}{\operatorname{Rey}}\left\{a^{2}(1-\xi)^{2} \frac{\partial^{2} \omega}{\partial \xi^{2}}-a^{2}(1-\xi) \frac{\partial \omega}{\partial \xi}+b^{2}(1-n)^{2} \frac{\partial^{2} \omega}{\partial n^{2}}\right. \\
\left.-b^{2}(1-n) \frac{\partial \omega}{\partial n}-\frac{\omega}{x^{2}}+\frac{b(1-n)}{x} \frac{\partial \omega}{\partial n}\right\}
\end{gathered}
$$

The central difference expressions to be used throughout this appendix have already been derived in Appendix $\mathrm{C}$ along with their accompanying truncation errors. Sample terms are given below. 


$$
\begin{gathered}
\frac{\partial \omega}{\partial \xi}=\frac{\omega(i+1, j)-\omega(i-1, j)}{\left(\frac{1}{\bar{M}}\right)} \\
\frac{\partial^{2} \omega}{\partial \xi^{2}}=\frac{\omega(i+1, j)+\omega(i-1, j)-2 \omega(i, j)}{\left(\frac{1}{M}\right)^{2}} \\
\frac{\partial \omega}{\partial \eta}=\frac{\omega(i, j+1)-\omega(i, j-1)}{\left(\frac{1}{M}\right)^{2}} \\
\frac{\partial^{2} \omega}{\partial \eta^{2}}=\frac{\omega(i, j+1)+\omega(i, j-1)-2 \omega(i, j)}{\left(\frac{1}{\bar{M}}\right)^{2}}
\end{gathered}
$$

where $\frac{1}{M}$ is the mesh spacing for a square region of the $\xi, n$ plane composed of $(M+1)^{2}$ equally spaced grid points.

For the moment, the time derivative of Equation (E-I) will be neglected. Remembering that in the transformed plane

$$
\xi=\frac{i}{M} \quad \text { and } \quad \eta=\frac{j}{M}
$$

the application to Equation $(E-I)$ of the central differences given above produces the expression 


$$
\begin{aligned}
& \frac{\partial \omega}{\partial t}=-\left\{a v_{z}(i, j)\left(\frac{M-i}{M}\right)\left[\frac{\omega(i+1, j)-\omega(i-1, j)}{2\left(\frac{1}{M}\right)}\right]\right. \\
& \left.+v_{r}(i, j)\left\langle b\left(\frac{M-j}{M}\right)\left[\frac{\omega(i, j+1)-\omega(i, j-1)}{2\left(\frac{I}{M}\right)}\right]-\frac{\omega(i, j)}{x}\right\rangle\right\} \\
& +\frac{1}{R e y}\left\{a^{2}\left(\frac{M-i}{M}\right)^{2}\left[\frac{\omega(i+1, j)+\omega(i-1, j)-2 \omega(i, j)}{\left(\frac{1}{M}\right)^{2}}\right]\right. \\
& -a^{2}\left(\frac{M-i}{M}\right)\left[\frac{\omega(i+1, j)-\omega(i-1, j)}{2\left(\frac{1}{M}\right)}\right] \\
& +\frac{b}{x}\left(\frac{M-j}{M}\right)\left[\frac{\omega(i, j+1)-\omega(i, j-1)}{2\left(\frac{1}{M}\right)}\right]-\frac{\omega(i, j)}{x^{2}}
\end{aligned}
$$

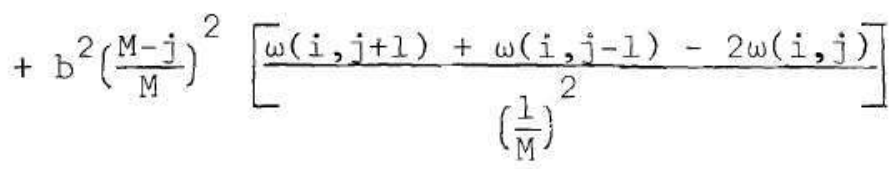

$$
\begin{aligned}
& \left.-b^{2}\left(\frac{M-j}{M}\right)\left[\frac{\omega(i, j+1)-\omega(i, j-1)}{2\left(\frac{1}{M}\right)}\right]\right\}
\end{aligned}
$$

where

$$
\begin{aligned}
& 1-\xi=1-\frac{i}{M}=\frac{M-i}{M} \\
& 1-n=1-\frac{j}{M}=\frac{M-j}{M}
\end{aligned}
$$


Equation (E-2) is recognized as Equation ( $D-4)$ of Appendix D, and it is the starting point for Case I through Case IV of that appendix. Simplifying Equation (E-2) and gathering like terms yields

$$
\begin{aligned}
& \frac{\partial \omega}{\partial t}=\left\{v_{z}(i, j) \frac{a(M-i)}{2}+\frac{a^{2}(M-i)}{\operatorname{Rey}}\left[(M-i)+\frac{1}{2}\right]\right\} \omega(i-1, j) \\
& +\left\{-v_{z}(i, j) \frac{a(M-i)}{2}+\frac{a^{2}(M-i)}{\operatorname{Rey}}\left[(M-i)-\frac{1}{2}\right]\right\} \omega(i+1, j) \\
& +\left\{v_{r}(i, j) \frac{b(M-j)}{2}+\frac{b^{2}(M-j)}{\operatorname{Rey}}\left[(M-j)+\frac{1}{2}\left(1-\frac{1}{b x}\right)\right]\right\} \omega(i, j-1) \\
& +\left\{-v_{r}(i, j) \frac{b(M-j)}{2}+\frac{b^{2}(M-j)}{\operatorname{Rey}}\left[(M-j)-\frac{1}{2}\left(1-\frac{1}{b x}\right)\right]\right\} \omega(i, j+1) \\
& -\frac{1}{\operatorname{Rey}}\left\{2 a^{2}(M-i)^{2}+2 b^{2}(M-j)^{2}-\frac{v_{r}(i, j) \operatorname{Rey}}{x}+\frac{1}{x^{2}}\right\} \omega(i, j)
\end{aligned}
$$

where

$$
x=r=\frac{1}{b} \ln \left(\frac{M}{M-j}\right)
$$

Equation $(E-3)$ is the starting point for the derivation of all finite difference equations which are the approximations to the simplified VTE, Equation $(E-1)$. 


\section{Case I. Forward Time Difference--Explicit}

\section{Formulation of the Simplified VTE}

In this approximation, the derivative of the vorticity with respect to time is given by

$$
\frac{\partial \omega}{\partial t}=\frac{\omega^{n+1}(i, j)-\omega^{n}(i, j)}{\delta t}
$$

All the terms on the right-hand side of Equation (E-3) are evaluated at the time $(n) \delta t$. Combining Equations $(E-3)$ and $(E-4)$ and simplifying gives

$$
\begin{aligned}
& \omega^{n+1}(i, j)=\delta t\left\langle\left\{\frac{1}{\delta t}-\frac{1}{R e y}\left[2 a^{2}(M-i)^{2}+2 b^{2}(M-j)^{2}\right.\right.\right. \\
& \left.\left.-\frac{v_{r}^{n}(i, j) R e y}{x}+\frac{1}{x^{2}}\right]\right\} \omega^{n}(i, j) \\
& +\left\{v_{z}^{n}(i, j) \frac{a(M-i)}{2}+\frac{a^{2}(M-i)}{R e y}\left[(M-i)+\frac{1}{2}\right]\right\} \omega^{n}(i-1, j) \\
& +\left\{-v_{z}^{n}(i, j) \frac{a(M-i)}{2}+\frac{a^{2}(M-i)}{R e y}\left[(M-i)-\frac{1}{2}\right]\right\} \omega^{n}(i+1, j) \\
& +\left\{v_{r}^{n}(i, j) \frac{b(M-j)}{2}+\frac{b^{2}(M-j)}{\left.\operatorname{Rey}\left[(M-j)+\frac{1}{2}\left(1-\frac{1}{b x}\right)\right]\right\} \omega^{n}(i, j-1)}\right. \\
& \left.+\left\{-v_{r}^{n}(i, j) \frac{b(M-j)}{2}+\frac{b^{2}(M-j)}{\operatorname{Rey}}\left[(M-j)-\frac{1}{2}\left(1-\frac{1}{b x}\right)\right]\right\} \omega^{n}(i, j+1)\right\rangle
\end{aligned}
$$


where

$$
\begin{aligned}
& v_{z}^{n}(i, j)=\frac{b(M-j)}{2 x}\left[\psi^{n}(i, j+1)-\psi^{n}(i, j-1)\right] \\
& v_{n}^{n}(i, j)=-\frac{a(M-i)}{2 x}\left[\psi^{n}(i+1, j)-\psi^{n}(i-1, j)\right]
\end{aligned}
$$

\section{Case II. Central Time Difference--Explicit}

\section{Formulation of the Simplified VTE}

The time derivative is approximated here by the expression

$$
\frac{\partial \omega}{\partial t}=\frac{\omega^{n+1}(i, j)-\omega^{n-1}(i, j)}{2 \delta t}
$$

The terms on the right-hand side of Equation (E-3) are again evaluated at the time $(n) \delta t$. Combining Equations $(E-3)$ and $(E-8)$ and simplifying gives

$$
\begin{aligned}
& \omega^{n+1}(i, j)=\omega^{n-1}(i, j)+2 \delta t\left\langle\left\{-\frac{1}{\operatorname{Rey}}\left[2 a^{2}(M-i)^{2}\right.\right.\right. \\
& \left.\left.+2 b^{2}(M-j)^{2}-\frac{v_{n}^{n}(i, j) R e y}{x}+\frac{1}{x^{2}}\right]\right\} \omega^{n}(i, j) \\
& +\left\{v_{z}^{n}(i, j) \frac{a(M-i)}{2}+\frac{a^{2}(M-i)}{\operatorname{Rey}}\left[(M-i)+\frac{1}{2}\right]\right\} \omega^{n}(i-1, j) \\
& +\left\{-v_{z}^{n}(i, j) \frac{a(M-i)}{2}+\frac{a^{2}(M-i)}{\operatorname{Rey}}\left[(M-i)-\frac{1}{2}\right]\right\} \omega^{n}(i+1, j)
\end{aligned}
$$




$$
\begin{aligned}
& +\left\{v_{r}^{n} \frac{b(M-j)}{2}+\frac{b^{2}(M-j)}{R e y}\left[(M-j)+\frac{1}{2}\left(1-\frac{1}{b x}\right)\right]\right\} \omega^{n}(i, j-1) \\
& +\left\{-v_{r}^{n} \frac{b(M-j)}{2}+\frac{b^{2}(M-j)}{R e y}\left[(M-j)-\frac{1}{2}\left(1-\frac{1}{b x}\right)\right]\right\} \omega^{n}(i, j+1)
\end{aligned}
$$

where

$$
\begin{aligned}
& v_{z}^{n}(i, j)=\frac{b(M-j)}{2 x}\left[\psi^{n}(i, j+1)-\psi^{n}(i, j-1)\right] \\
& v_{n}^{n}(i, j)=-\frac{a(M-i)}{2 x}\left[\psi^{n}(i+1, j)-\psi^{n}(i-1, j)\right]
\end{aligned}
$$

Case III. Central Time Difference--Explicit Formulation of the Simplified VTE (Time Average Technique)

The time derivative is again approximated by the central difference

$$
\frac{\partial \omega}{\partial t}=\frac{\omega^{n+1}(i, j)-\omega^{n-1}(i, j)}{2 \delta t}
$$

but the vorticity term $\omega(i, j)$ is approximated by a time average to give the equation

$$
\frac{1}{\operatorname{Rey}}\left\{2 a^{2}(M-i)^{2}+2 b^{2}(M-j)^{2}+\frac{1}{x^{2}}-\frac{v_{r}^{n}(i, j) \text { Rey }}{x}\right\} \omega(i, j)=(E-13)
$$




$$
\begin{gathered}
\frac{1}{2 \operatorname{Rey}}\left\{2 a^{2}(M-i)^{2}+2 b^{2}(M-j)^{2}+\frac{1}{x^{2}}-\frac{v_{r}^{n}(i, j) \operatorname{Rey}}{x}\right\} \\
{\left[\omega^{n+1}(i, j)+\omega^{n-1}(i, j)\right]}
\end{gathered}
$$

Combining Equations $(E-3),(E-12)$, and $(E-13)$ and simplifying the resulting expression produces the equation

$$
\begin{aligned}
& \omega^{n+1}(i, j)=\left\{\frac{1}{2 \delta t}+\frac{1}{2 \operatorname{Rey}}\left[2 a^{2}(M-i)^{2}+2 b^{2}(M-j)^{2}\right.\right. \\
& \left.\left.+\frac{v_{r}^{n}(i, j) \operatorname{Rey}}{x}-\frac{1}{x^{2}}\right]\right\}^{-1} \\
& \left\langle\left\{\frac{1}{2 \delta t}-\frac{1}{2 \operatorname{Rey}}\left[2 a^{2}(M-i)^{2}+2 b^{2}(M-j)^{2}\right.\right.\right. \\
& \left.\left.+\frac{v_{r}^{n}(i, j) \operatorname{Rey}}{x}-\frac{1}{x^{2}}\right]\right\} \omega^{n-1}(i, j) \\
& +\left\{v_{z}^{n}(i, j) \frac{a(M-i)}{2}+\frac{a^{2}(M-i)}{\operatorname{Rey}}\left[(M-i)+\frac{1}{2}\right]\right\} \omega^{n}(i-1, j) \\
& +\left\{v_{r}^{n}(i, j) \frac{b(M-j)}{2}+\frac{b^{2}(M-j)}{\operatorname{Rey}}\left[(M-j)+\frac{1}{2}\right]\right\} \omega^{n}(i, j-1) \\
& +\left\{-v_{z}^{n}(i, j) \frac{a(M-i)}{2}+\frac{a^{2}(M-i)}{\operatorname{Rey}}\left[(M-i)-\frac{1}{2}\right]\right\} \omega^{n}(i+1, j)
\end{aligned}
$$




$$
+\left\{-v_{r}^{n}(i, j) \frac{b(M-j)}{2}+\frac{b^{2}(M-j)}{R e y}\left[(M-j)-\frac{I}{2}\right]\right\} \omega^{n}(i, j+I)
$$

where

$$
\begin{aligned}
& v_{z}^{n}(i, j)=\frac{b(M-j)}{2 x}\left[\psi^{n}(i, j+1)-\psi^{n}(i, j-1)\right] \\
& v_{r}^{n}(i, j)=-\frac{a(M-i)}{2 x}\left[\psi^{n}(i+1, j)-\psi^{n}(i-1, j)\right]
\end{aligned}
$$

\section{Case IV. Forward Time Difference--Implicit}

\section{Formulation of the Simplified VTE}

In this formulation, the time derivative of Equation $(E-3)$ is approximated by

$$
\frac{\partial \omega}{\partial t}=\frac{\omega^{n+1}(i, j)-\omega^{n}(i, j)}{\delta t}
$$

and the terms on the right-hand side of Equation $(E-3)$ are evaluated at the time $\left(n+\frac{1}{2}\right) \delta t$ which is approximated by the time average technique. A sample term is given by

$$
\omega^{n+1 / 2}(i+1, j)=\frac{1}{2}\left\{\omega^{n+1}(i+1, j)+\omega^{n}(i+1, j)\right\}
$$

Combining Equations $(E-3)$ and (E-17), applying the time average, and reducing the resulting equation gives 


$$
\begin{aligned}
& \omega^{n+1}(i, j)=\left\{\frac{1}{\delta t}+\frac{1}{2 \operatorname{Rey}}\left[2 a^{2}(M-i)^{2}+2 b^{2}(M-j)^{2}\right.\right. \\
& \left.\left.-\frac{v_{r}^{n+1 / 2}(i, j) R e y}{x}+\frac{1}{x^{2}}\right]\right\}^{-1} \\
& \left\langle\left\{\frac{1}{\delta t}-\frac{1}{2 \operatorname{Rey}}\left[2 a^{2}(M-i)^{2}+2 b^{2}(M-j)^{2}-\frac{v_{r}^{n+1 / 2}(i, j) \operatorname{Rey}}{x}+\frac{1}{x^{2}}\right]\right\} \omega^{n}(i, j)\right. \\
& +\left\{-v_{z}^{n+1 / 2}(i, j) \frac{a(M-i)}{2}+\frac{a^{2}(M-i)}{R e y}\left[(M-i)-\frac{1}{2}\right]\right\} \\
& \left\{\frac{1}{2}\left[\omega^{n+1}(i+1, j)+\omega^{n}(i+1, j)\right]\right\} \\
& +\left\{v_{r}^{n+1 / 2}(i, j) \frac{b(M-j)}{2}+\frac{b^{2}(M-j)}{R e y}\left[(M-j)+\frac{1}{2}\left(1-\frac{1}{b x}\right)\right]\right\} \\
& \left\{\frac{1}{2}\left[\omega^{n+1}(i, j-1)+\omega^{n}(i, j-1)\right]\right\} \\
& +\left\{-v_{r}^{n+1 / 2}(i, j) \frac{b(M-j)}{2}+\frac{b^{2}(M-j)}{R e y}\left[(M-j)-\frac{1}{2}\left(1-\frac{1}{b x}\right)\right]\right\} \\
& +\left\{v_{z}^{n+1 / 2}(i, j) \frac{a(M-i)}{2}+\frac{a^{2}(M-i)}{R e y}\left[(M-i)+\frac{1}{2}\right]\right\} \frac{1}{2}\left\{\left[\omega^{n+1}(i-1, j)+\omega^{n}(i-1, j)\right]\right\}
\end{aligned}
$$




$$
\left\{\frac{1}{2}\left[\omega^{n+1}(i, j+1)+\omega^{n}(i, j+1)\right]\right\}
$$

where

$$
\begin{aligned}
& v_{z}^{n+1 / 2}(i, j)=\frac{b(M-j)}{4 x}\left\{\psi^{n+1}(i, j+1)+\psi^{n}(i, j+1)\right. \\
& \left.-\psi^{n+1}(i, j-1)-\psi^{n}(i, j-1)\right\} \\
& v_{r}^{n+1 / 2}(i, j)=-\frac{a(M-i)}{4 x}\left\{\psi^{n+1 / 2}(i+1, j)+\psi^{n}(i+1, j)\right. \\
& \left.-\psi^{n+1}(i-1, j)-\psi^{n}(i-1, j)\right\}
\end{aligned}
$$

The Unsimplified VTE

Equation (31) is repeated here, and it is written

$$
\begin{aligned}
& \frac{\partial \omega}{\partial t}+a(1-\xi) \frac{\partial\left(v_{z} \omega\right)}{\partial \xi}+b(1-n) \frac{\partial\left(v_{n} \omega\right)}{\partial \eta}= \\
& \frac{1}{\operatorname{Rey}}\left\{a^{2}(1-\xi)^{2} \frac{\partial^{2} \omega}{\partial \xi^{2}}-a^{2}(1-\xi) \frac{\partial \omega}{\partial \xi}+b^{2}(1-n)^{2} \frac{\partial^{2} \omega}{\partial \eta^{2}}-b^{2}(1-n) \frac{\partial \omega}{\partial \eta}\right. \\
& \left.+\frac{b(l-n)}{x} \frac{\partial \omega}{\partial \eta}-\frac{\omega}{x^{2}}\right]
\end{aligned}
$$

Applying the central differences illustrated earlier to Equation (E-2I) and simplifying the result yields 


$$
\begin{aligned}
& \frac{\partial \omega}{\partial t}=-\frac{a(M-i)}{2}\left[v_{z}(i+1, j) \omega(i+1, j)-v_{z}(i-1, j) \omega(i-1, j)\right] \\
& -\frac{b(M-j)}{2}\left[v_{r}(i, j+1) \omega(i, j+1)-v_{r}(i, j-1) \omega(i, j-1)\right] \\
& +\frac{1}{\operatorname{Rey}}\left\{a^{2}(M-i)^{2}[\omega(i+1, j)+\omega(i-1, j)]-\frac{a^{2}(M-i)}{2}[\omega(i+1, j)-\omega(i-1, j)]\right. \\
& +b^{2}(M-j)^{2}[\omega(i, j+1)+\omega(i, j-1)]-\frac{b^{2}(M-j)}{2}[\omega(i, j+1)-\omega(i, j-1)] \\
& \left.+\frac{b(M-j)}{2 x}[\omega(i, j+1)-\omega(i, j-1)]\right\} \\
& -\frac{1}{\operatorname{Rey}}\left\{2 a^{2}(M-i)^{2}+2 b^{2}(M-j)^{2}+\frac{1}{x^{2}}\right\} \omega(i, j)
\end{aligned}
$$

This equation corresponds to Equation $(D-43)$ of Appendix $D$, and it is the starting point for the stability analysis of Case $V$ of that appendix.

\section{Case V. Forward Time Difference--}

Implicit Formulation of the Unsimplified VTE

Here, the forward time difference is employed, and this gives the finite difference approximation

$$
\frac{\partial \omega}{\partial t}=\frac{\omega^{n+1}(i, j)-\omega^{n}(i, j)}{\delta t}
$$

Combining Equations $(E-22)$ and $(E-23)$ and gathering like terms of the result gives after some simplification, 


$$
\begin{aligned}
& \frac{\omega^{n+1}(i, j)-\omega^{n}(i, j)}{\delta t}=-\frac{1}{\operatorname{Rey}}\left\{2 a^{2}(M-i)^{2}\right. \\
& \left.+2 b^{2}(M-j)^{2}+\frac{1}{x^{2}}\right\} \omega(i, j) \\
& +\left\{v_{z}(i-1, j) \frac{a(M-i)}{2}+\frac{a^{2}(M-i)}{R e y}\left[(M-i)+\frac{1}{2}\right\} \omega(i-1, j)\right. \\
& +\left\{-v_{z}(i+1, j) \frac{a(M-i)}{2}+\frac{a^{2}(M-i)}{R e y}\left[(M-i)-\frac{1}{2}\right]\right\} \omega(i+1, j) \\
& +\left\{v_{r}(i, j-1) \frac{b(M-j)}{2}+\frac{b^{2}(M-j)}{R e y}\left[(M-j)+\frac{1}{2}\left(1-\frac{1}{b x}\right\}\right\} \omega(i, j-1)\right. \\
& \left.+\frac{b(M-j)}{2}+\frac{b^{2}(M-j)}{R e y}\left[(M-j)-\frac{1}{2}\left(1-\frac{1}{b x}\right)\right]\right\} \omega(i, j+1)
\end{aligned}
$$

All the variables on the right-hand side of this equation are evaluated at the time $\left(n+\frac{1}{2}\right) \delta t$. The time average technique is employed to give an implicit statement of the VTE. A sample term of the time average is

$$
\omega^{n+1 / 2}(i, j)=\frac{1}{2}\left\{\omega^{n+1}(i, j)+\omega^{n}(i, j)\right\}
$$

Applying the time averages to the terms of Equation (E-24) and reducing the resulting expression gives

$$
\omega^{n+1}(i, j)=\left\{\frac{1}{\delta t}+\frac{1}{R e y}\left[a^{2}(M-i)^{2}+b^{2}(M-j)^{2}+\frac{1}{2 x^{2}}\right]\right\}^{-1}
$$




$$
\begin{aligned}
& \left\langle\left\{\frac{1}{\delta t}-\frac{1}{R e y}\left[a^{2}(M-i)^{2}+b^{2}(M-j)^{2}+\frac{1}{2 x^{2}}\right]\right\} \omega^{n}(i, j)\right. \\
& +\left\{\frac{a(M-i) v_{z}^{n+l / 2}(i-1, j)}{2}+\frac{a^{2}(M-i)}{R e y}\left[(M-i)+\frac{1}{2}\right]\right\} \\
& \frac{1}{2}\left\{\omega^{n+1}(i-1, j)+\omega^{n}(i-1, j)\right\} \\
& +\left\{\frac{-a(M-i) v_{z}^{n+l / 2}(i+1, j)}{2}+\frac{a^{2}(M-i)}{R e y}\left[(M-i)-\frac{1}{2}\right]\right\} \\
& \frac{1}{2}\left\{\omega^{n+1}(i+1, j)+\omega^{n}(i+1, j)\right\} \\
& +\left\{\frac{b(M-j) v_{r}^{n+l / 2}(i, j-1)}{2}+\frac{b^{2}(M-j)}{R e y}\left[(M-j)+\frac{1}{2}\left(1-\frac{1}{b x}\right)\right]\right\} \\
& \frac{1}{2}\left\{\omega^{n+1}(i, j-1)+\omega^{n}(i, j-1)\right\} \\
& +\left\{\frac{-b(M-j) v_{r}^{n+1 / 2}(i, j+1)}{2}+\frac{b^{2}(M-j)}{R e y}\left[(M-j)-\frac{1}{2}\left(1-\frac{1}{b x}\right)\right]\right\} \\
& \frac{1}{2}\left\{\omega^{n+1}(i, j+1)+\omega^{n}(i, j+1)\right\}
\end{aligned}
$$

where

$$
v_{z}^{n+1 / 2}(i-1, j)=\frac{b(M-j)}{4 x}\left[\psi^{n+1}(i-1, j+1)+\psi^{n}(i-1, j+1)\right.
$$




$$
\begin{aligned}
&-\left.\psi^{n+1}(i-1, j-1)-\psi^{n}(i-1, j-1)\right] \\
& v_{z}^{n+1 / 2}(i+1, j)=\frac{b(M-j)}{4 x}\left[\psi^{n+1}(i+1, j+1)+\psi^{n}(i+1, j+1)\right. \\
&-\left.\psi^{n+1}(i+1, j-1)-\psi^{n}(i-1, j-1)\right] \\
& v_{r}^{n+1 / 2}(i, j-1)=-\frac{a(M-i)}{4 x}\left[\psi^{n+1}(i+1, j-1)+\psi^{n}(i+1, j-1)\right. \\
&-\left.\psi^{n+1}(i-1, j-1)-\psi^{n+1}(i-1, j-1)\right] \\
& v_{r}^{n+1 / 2}(i, j+1)=-\frac{a(M-i)}{4 x}\left[\psi^{n+1}(i+1, j+1)+\psi^{n}(i+1, j+1)\right. \\
&\left.-\psi^{n+1}(i-1, j+1)-\psi^{n}(i-1, j+1)\right]
\end{aligned}
$$

Equation (E-25) corresponds to Equation (48) of the text. The method of solution of the system of equations arising from the application of Equation (E-24) of each interior mesh point is given in the discussion of numerical methods in Appendix G.

This concludes the derivations of the various finite difference approximations of the VTE. No motivation concerning the reasons why these equations are needed in these particular forms has been given. The logic of this development has already been discussed in detail in the stability analyses of these equations in Appendix D. 


\section{APPENDIX F \\ FINITE DIFFERENCE FORM OF THE \\ DEFINITION OF VORTICITY EQUATION}

The differential equation describing the vorticity in terms of the stream function in the transformed plane is given in the text as Equation (32), and it may be written

$$
\begin{gathered}
\frac{1}{x}\left\{a^{2}(1-\xi)^{2} \frac{\partial^{2} \psi}{\partial \xi^{2}}-a^{2}(1-\xi) \frac{\partial \psi}{\partial \xi}\right\}-\frac{1}{x^{2}}\left\{b(1-n) \frac{\partial \psi}{\partial n}\right\} \\
+\frac{1}{x}\left\{b^{2}(1-n)^{2} \frac{\partial^{2} \psi}{\partial n^{2}}-b^{2}(1-n) \frac{\partial \psi}{\partial n}\right\}=-\omega
\end{gathered}
$$

Since this equation is never applied at the boundaries, it may be multiplied through by the quantity $x$. Applying the central differences of Appendix $C$ to the partial derivatives evaluated at the point

$$
\xi=\frac{i}{M} \quad n=\frac{j}{M}
$$

where again $\left(\frac{1}{M}\right)$ is the mesh spacing yields the equation

$$
\left\{a^{2}\left(\frac{M-i}{M}\right)^{2}\left[\frac{\psi(i+1, j)+\psi(i-1, j)-2 \psi(i, j)}{\left(\frac{1}{M}\right)^{2}}\right]\right.
$$




$$
\begin{aligned}
& \left.-a^{2}\left(\frac{M-i}{M}\right)\left[\frac{\psi(i+1, j)-\psi(i-1, j)}{2\left(\frac{1}{M}\right)}\right]\right\} \\
& -\frac{1}{x}\left\{b\left(\frac{M-j}{M}\right)\left[\frac{\psi(i, j+1)-\psi(i, j-1)}{2\left(\frac{1}{M}\right)}\right]\right\} \\
& +\left\{b^{2}\left(\frac{M-j}{M}\right)^{2}\left[\frac{\psi(i, j+1)+\psi(i, j-1)-2 \psi(i, j)}{2}\right]\right. \\
& -b^{2}\left(\frac{M-j}{M}\right)\left[\frac{\left(\frac{1}{M}\right)^{2}}{2\left(\frac{1}{M}\right)}\left[\frac{\psi(i, j-1)}{M}\right]=-\omega x\right.
\end{aligned}
$$

Simplifying this equation and transposing the term $\omega x$ and the term containing $\psi(i, j)$ produces

$$
\begin{aligned}
& {\left[2 a^{2}(M-i)^{2}+2 b^{2}(M-j)^{2}\right] \psi(i, j)=w x-\frac{b(M-j)}{2 x}[\psi(i, j+1)-\psi(i, j-1)] } \\
+ & \left\{a^{2}(M-i)^{2}[\psi(i+1, j)+\psi(i-1, j)]-\frac{a^{2}(M-i)}{2}[\psi(i+1, j)-\psi(i-1, j)]\right\} \\
+ & \left\{b^{2}(M-j)^{2}[\psi(i, j+1)+\psi(i, j-1)]-\frac{b^{2}(M-j)}{2}[\psi(i, j+1)-\psi(i, j-1)]\right\}
\end{aligned}
$$

Gathering like terms and denoting the time at $t=(n+1) \delta t$ by the time step index $(n+l)$ gives finally

$$
\psi^{n+1}(i, j)=\left\langlea ^ { 2 } ( M - i ) \left\{\left[(M-i)-\frac{i}{2} \mid \psi^{n+1}(i+1, j)+\left[(M-i)+\frac{1}{2}\right] \psi^{n+1}(i-1, j)\right\}(E-4)\right.\right.
$$




$$
\begin{aligned}
& +b^{2}(M-j)\left\{\left[(M-j)-\frac{1}{2}\left(1+\frac{1}{b x}\right)\right] \psi^{n+1}(i, j+1)\right. \\
& +\left[(M-j)+\frac{1}{2}\left(1+\frac{1}{b x}\right)\right] \psi^{n+1}(i, j-1) \\
& \left.+x \omega^{n+1}(i, j)\right\rangle\left\{2 a^{2}(M-i)^{2}+2 b^{2}(M-j)^{2}\right\}^{-1}
\end{aligned}
$$

where

$$
x=r=\frac{1}{b} \ln \left(\frac{1}{1-n}\right)=\frac{1}{b} \ln \left(\frac{M}{M-j}\right)
$$

This is Equation (62) of the text.

It should be noted here that this equation changes slightly at the mesh points just inside the boundaries of $\xi=1$ and $n=1$ for which $i=M$ and $j=M$. For the point $i=M-1$, the Neumann condition states that

$$
\psi^{n+1}(M-1, j)=\psi^{n+1}(M, j)
$$

Hence, at this point

$$
\psi^{n+1}(i+1, j)=\psi^{n+1}(i, j)
$$

so Equation $(\mathrm{F}-4)$ simplifies to the form

$$
\psi^{n+1}(i, j)=\left\langle a^{2}(M-i)\left[(M-i)+\frac{1}{2}\right] \psi^{n+1}(i-1, j)\right.
$$




$$
\begin{gathered}
+b^{2}(M-j)\left\{\left[(M-j)-\frac{1}{2}\left(1+\frac{1}{b x}\right)\right] \psi^{n+1}(i, j+1)\right. \\
\left.+\left[(M-j)+\frac{1}{2}\left(1+\frac{1}{b x}\right)\right] \psi^{n+1}(i, j-1)\right\} \\
\left.+x \omega^{n+1}(i, j)\right\rangle\left\{2 a^{2}(M-i)^{2}+2 b^{2}(M-j)^{2}-a^{2}(M-i)^{2}\left[(M-i)-\frac{1}{2}\right]\right\}^{-1}
\end{gathered}
$$

Similarly, at the point $j=M-1$, for which

$$
\psi^{n+1}(i, M-1)=\psi^{n+1}(i, M)
$$

and hence

$$
\psi^{n+1}(i, j+1)=\psi^{n+1}(i, j)
$$

Equation $(\mathrm{F}-4)$ simplifies to the form

$$
\begin{aligned}
& \psi^{n+1}(i, j)=\left\langlea ^ { 2 } ( M - i ) \left\{\left[(M-i)-\frac{1}{2}\right] \psi^{n+1}(i+1, j)\right.\right. \\
&+ {\left.\left[(M-i)+\frac{1}{2}\right] \psi^{n+1}(i-1, j)\right\} } \\
&+b^{2}(M-j) {\left[(M-j)+\frac{1}{2}\left(1+\frac{1}{b x}\right)\right] \psi^{n+1}(i, j-1) } \\
&\left.+x \omega^{n+1}(i, j)\right\rangle\left\{2 a^{2}(M-i)^{2}+2 b^{2}(M-j)^{2}\right.
\end{aligned}
$$




$$
\left.-b^{2}(M-j)\left[(M-j)-\frac{1}{2}\left(1+\frac{1}{b x}\right)\right]\right\}^{-1}
$$

This completes the finite difference approximation to the DVE. 
APPENDIX G

\author{
SOME ITERATIVE METHODS FOR THE NUMERICAL \\ SOLUTION OF ELLIPTIC PARTIAL DIFFERENTIAL EQUATIONS
}

The numerical model for both the implicit VTE and the DVE requires the solution of a system composed of a large number of simultaneous algebraic equations. Each algebraic equation has its origin in the application of the pertinent finite difference equation to a particular grid point. Since a closed form solution to such a system is intractable, an iterative technique is required. However, the question of the convergence of a particular technique is based to a large extent on a knowledge of the eigenvalues of the matrix $A$ in the system

$$
A \bar{\phi}=B
$$

where $\bar{\phi}$ is the column vector of the dependent variable. For a large and complicated matrix $A$, the difficulty in finding the eigenvalues is of the same order of magnitude as that encountered in finding the dependent variable. Hence, it appears that the most expedient way to see whether or not an iterative technique will work is to try it. No recommendations will be made as to which of the following methods will provide the fastest solution in terms of machine time for a given accuracy and for a particular equation. Only the successful applications in this investigation for the VTE and the DVE will be noted. 
Both of the finite difference equations mentioned above may be written in the form

$$
\begin{aligned}
& \phi(i, j)=k_{1} \phi(i-1, j)+k_{2} \phi(i+1, j) \\
& +k_{3} \phi(i, j-1)+k_{4} \phi(i, j+1)+k_{5}
\end{aligned}
$$

where, in functional notation,

$$
K_{k}=K_{k}(i, j, M \text {, parameters of the flow })
$$

This is a fairly general form which should apply to second order equations which are approximated by central differences. In the ensuing discussion, the iterative method will sweep the mesh along rows or columns in the direction of increasing values of the indices $i$ and $j$. In this manner, the latest values of $\phi(i-1, j)$ and $\phi(i, j-1)$ will have been calculated before $\phi(i, j)$ is calculated.

\section{Method I. Jacobi's Method}

From the initial approximation $\phi^{0}(i, j)$, improved values of $\phi(i, j)$ may be calculated over the interior points of the mesh by employing the recursive relation

$$
\phi^{m+1}=k_{1} \phi^{m}(i-1, j)+k_{2} \phi^{m}(i+1, j)+k_{3} \phi^{m}(i, j-1)+k_{4} \phi^{m}(i, j+1)+k_{5}
$$

where the superscript $m$ refers to the number of the iteration. 
A complete iteration consists of sweeping the entire region of interest, and the iteration is repeated until the difference between the successive iterates $\phi^{m+1}(i, j)$ and $\phi^{m}(i, j)$ is less than some predetermined tolerance throughout the flow field. Slowness to converge is the primary disadvantage to this method, and the time to converge becomes larger as the number of mesh points is increased.

Method II. The Gauss-Seidel or Liebmann Method

This method is known by the former name when it's applied to systems of algebraic equations and by the latter when it's applied to partial differential equations. This appears to be paradoxical. The advantage to this method over Jacobi's method is that use is made of the newest values of the dependent variable. This is shown in the recursive relation

$$
\begin{aligned}
& \phi^{m+1}(i, j)=k_{1} \phi^{m+1}(i-1, j)+k_{2} \phi^{m}(i+1, j) \\
& +k_{3} \phi^{m+1}(i, j-1)+k_{4} \phi^{m}(i, j+1)+k_{5}
\end{aligned}
$$

Again, one iteration consists of a complete sweep of the field, and iterating is continued until. the difference between successive iterates becomes small. According to Faddeeva [39] three things may occur when Methods I and II are applied to the same system of equations. Depending on the eigenvalues of the matrix $A$ in Equation (G-1), (1) Method II may be a substantial improvement upon Method I, (2) Method II may diverge although Method I has converged, or (3) Method II may converge although 
Method I has diverged. For case (I), Method II offers another slight advantage over Method I because the computer does not have to keep track of old and new values of $\phi(i, j)$. It simply replaces the old value with the latest guess.

Method III. The Method of Successive Over-Relaxation

This method is often referred to in a shorthand notation as the S.O.R. method. When applied in conjunction with Liebmann's method, this procedure speeds convergence by correcting the latest iterate through the use of an acceleration or relaxation factor $R$ where $I<R<2$ for over-relaxation.

Assume that the Liebmann method has been demonstrated to converge for the finite difference approximation represented by Equation (G-3). Since successive iterates for $\phi(i, j)$ are approaching closer and closer to the exact numerical solution, the difference between these iterates should be getting smaller. By increasing this difference between approximations, the difference between each iterate and the solution is decreased, and the rate of convergence is accelerated. In this manner, a better approximation, $\tilde{\phi}(i, j)$, may be computed from the equation

$$
\tilde{\phi}(i, j)=\phi^{m}(i, j)+R\left[\phi^{m+1}(i, j)-\phi^{m}(i, j)\right]
$$

Equation (G-4) illustrates that the difference between successive iterates has been increased for $R>1$. Now, $\phi^{m+1}(i, j)$ has already been established in the Liebmann method by Equation $(G-3)$. Combining these two equations, replacing the quantity $\tilde{\phi}(i, j)$ by the standard notation 
for the new guess, $\phi^{m+1}(i, j)$, and simplifying gives the algorithm used in successive over-relaxation, i.e.

$$
\begin{gathered}
\phi^{m+1}(i, j)=R\left\{k_{1} \phi^{m+1}(i-1, j)+k_{2} \phi^{m}(i+1, j)\right. \\
\left.+k_{3} \phi^{m+1}(i, j-1)+k_{4} \phi^{m}(i, j+1)+k_{5}\right\} \\
-(R-1) \phi^{m}(i, j)
\end{gathered}
$$

When the acceleration factor takes the value of unity, this reduces to the Liebmann method represented by Equation (G-3), and when convergence has been achieved such that

$$
\phi^{m+1}(i, j) \cong \phi^{m}(i, j)
$$

the equation reduces to the original finite difference equation represented by Equation (G-2).

Although this method may converge in less than half the time it takes Method II, the calculation of $R$, in particular the optimum $R$, presents a complication. Although the optimum value of $R$ has been calculated for Laplace's equation on a square mesh by determining the largest eigenvalue of a matrix, this is of little general interest. More recently, Carre [40] has found a simpler method which is adaptable to computer solutions.

Some norm of the vector difference 


$$
T^{m}(i, j)=\phi^{m}(i, j)-\phi^{m-1}(i, j)
$$

may be calculated by the deviation of successive iterates. Various norms are:

$$
\begin{aligned}
& \|N\|_{\infty}=n^{-m}=\sum_{i=1}^{M-1} \max _{j}\left\{T^{m}(i, j)\right\} \\
& \|N\|_{1}=n^{-m}=\sum_{i=1}^{M-1} \sum_{j=1}^{M-1} T^{m}(i, j) \\
& \|N\|_{2}=n^{-m}=\sqrt{\sum_{i=1}^{M-1} \sum_{j=1}^{M-1}\left[T^{m}(i, j)\right]^{2}}
\end{aligned}
$$

It can be shown that

$$
\operatorname{limit}_{m \rightarrow \infty} \frac{n^{-m}}{n^{-m-1}}=\Lambda_{\max }
$$

where $\Lambda_{\max }$ is the largest eigenvalue of the vector $T^{m}(i, j)$. Thus, given an old guess at the relaxation factor, a new guess $R_{\mathrm{m}}$ for the optimum relaxation factor may be calculated using the relations

$$
R_{0}=2\left\{1+\sqrt{1-\frac{\left(\Lambda_{\max }+R-1\right)^{2}}{\Lambda_{\max }}}\right\}^{-1}
$$

and 


$$
R_{\mathrm{m}}=R_{0}-\frac{\left(2-R_{0}\right)}{4}
$$

The following procedure is recommended: (I) Sweep the field several times with $R=1$ to get a smooth start. (2) After setting $R=1.375$, calculate a new guess for $R_{m}$ every ten iterations. Once again, the iterative procedure has converged when successive approximations for $\phi(i, j)$ agree within some tolerance of the iteration.

\section{Method IV. Refining the Mesh}

The time to converge for each of the previous iterative methods is dependent upon the accuracy of the initial guess. It is sometimes quicker to solve the governing simultaneous equations at every other mesh point until convergence is achieved and then to calculate values at the intermediate points as averages of the surrounding points. In a given period of machine time, the combination of the solution over the courser mesh and the averaging technique may give a better first guess for the refined mesh than will the solution of the equations over the refined mesh.

\section{Method V. The Combination Method}

This method which was developed by experience combines the better features of Method III and Method IV. In its application to the DVE, this algorithm converged within a given tolerance to an approximate solution in about one-third the machine time (on the Burrough's B-5500) needed for the Liebmann method. This was faster than any of the other iterative procedures discussed thus far. The recursive relation is given 
by the equation

$$
\begin{aligned}
\phi^{m+1}(i, j)=R & \left\{k_{1} \phi^{m+1}(i-q, j)+k_{2} \phi^{m}(i+q, j)+k_{3} \phi^{m+1}(i, j-q)\right. \\
& \left.+k_{4} \phi^{m}(i, j+q)+k_{5}\right\}-(R-1) \phi^{m}(i, j)
\end{aligned}
$$

where $q$ is a dummy index which allows changing the mesh size. Basically, the idea is to iterate this equation over a number of finer and finer meshes all the while calculating the newest guess $R_{\mathrm{m}}$ for the optimum over-relaxation factor. More precisely, the procedure is as follows:

1. Perform several iterations with $R=1$ and $\mathrm{q}=4$ over $\mathrm{a}$ mesh consisting of every fifth grid point of the final mesh. The final mesh, the finest one, is the one for which the solution is to be found.

2. Set $R=1.375$ and continue iterating until convergence is reached. A new guess for $R_{\mathrm{m}}$ is to be calculated every ten iterations.

3. Refine the mesh by setting $q=2$ and calculating the dependent variable at every other mesh point of the final grid, and continue to calculate a new $R_{\mathrm{m}}$ every ten iterations. (Do not set $R_{\mathrm{m}}=1$ or $R_{m}=1.375$ when starting the iteration on this new mesh.) Continue until convergence is achieved.

4. Refine the mesh to its final spacing by setting $q=1$. Replace $R$ by a new guess at $R_{\mathrm{m}}$ every ten iterations until convergence is reached. (Again, do not restart the acceleration factor at $R_{\mathrm{m}}=I$ or $R_{\mathrm{m}}=1.375$. ) 
The following are a few remarks concerning specifically the application of Method $V$ to the DVE of this investigation. It was found (as expected) that for a particular number of mesh points $M$ the optimum over-relaxation factor did not vary with the values of $\phi(i, j)$. It was discovered that a cruder but sometimes faster method of calculating the optimum $R$ was to guess at several values of $R$ in the range $1<R<2$ and try them in the iteration over the finest grid. The iteration which converged the fastest contained a guessed $R$ which was closest to the optimum value. For example, for $M=24$ Method $V$ found the optimum relaxation factor to be $R=1.66$, whereas for $M=32$ the cruder approach found $R \cong 1.75$. Also, it was found that the iteration proceeded faster if the initial guess at $\phi(i, j)$ was whatever was left in the machine. This was of course the solution to the last complete iteration, and even if this corresponded to a previous time step, the iteration closed faster. If this were not the case, a little more time would have been necessary to zero these last values in the machine. Finally, once the optimum over-relaxation factor was known from the experience gained by Method V, the iteration using Equation (G-5) converged in about one-fifth of the time needed for Method II.

\section{Method VI. The Alternating Direction Method}

This method is a special case of a class of techniques called implicit line methods. The method to be described here has also come to be known as the Peaceman-Rachford method as it discussed in [41]. An excellent presentation of the method is given by Pearson [32]. 
By writing the recursive relation in the form

$$
\begin{gathered}
k_{1} \phi^{m+1}(i-1, j)-\phi^{m+1}(i, j)+k_{2} \phi^{m+1}(i+1, j)= \\
-k_{3} \phi^{m}(i, j-1)-k_{4} \phi^{m}(i, j+1)-k_{5}
\end{gathered}
$$

it is discovered that for a particular $j$ the solution of the tridiagonal matrix equation

$$
\left[\begin{array}{ccccccc}
-1 & k_{2} & 0 & 0 & \cdots & 0 & 0 \\
k_{1} & -1 & k_{2} & 0 & \cdots & 0 & 0 \\
0 & k_{1} & -1 & k_{2} & \cdots & 0 & 0 \\
\vdots & & & & & & \\
0 & 0 & 0 & 0 & \cdots & k_{1} & -1
\end{array}\right]\left[\begin{array}{c}
\phi(1, j) \\
\phi(2, j) \\
\vdots \\
\phi(3, j) \\
\phi(M-1, j)
\end{array}\right]=\left[\begin{array}{c}
-k_{3} \phi(1, j-1)-k_{4} \phi(1, j+1)-k_{5}-k_{1} \phi(0, j) \\
-k_{3} \phi(2, j-1)-k_{4} \phi(2, j+1)-k_{5} \\
-k_{3} \phi(3, j-1)-k_{4} \phi(3, j+1)-k^{\prime} \\
\vdots \\
-k_{3} \phi(M-1, j-1)-k_{4} \phi(M-1, j+1)-k_{5}-k_{2} \phi(M, j)
\end{array}\right]
$$

yields new iterates for all $\phi(i, j)$ 's for that value of $j$. By varying $j$ from $j=1$ to $j=M-1$, new iterates may be calculated over the entire mesh. As usual, the superscripts $m$ and $m+1$ refer to the iteration number; they should not be confused with the time step index $n$. For the case of the VTE, for example, the values of the vorticity and the stream function at the previous time step are lumped into the constant $K_{5}$ since they are no longer variables of the problem.

The recursive relation already presented may be supplanted by two of them of the form 


$$
\begin{gathered}
k_{1} \phi^{m+1 / 2}(i-1, j)-\phi^{m+1 / 2}(i, j)+k_{2} \phi^{m+1 / 2}(i+1, j)= \\
-k_{3} \phi^{m}(i, j-1)-k_{4} \phi^{m}(i, j+1)-k_{5}
\end{gathered}
$$

and

$$
\begin{aligned}
k_{3} \phi^{m+1}(i, j-1)-\phi^{m+1}(i, j)+k_{4} \phi^{m+1}(i, j+1) & = \\
& -k_{1} \phi^{m+1 / 2}(i-1, j)-k_{2} \phi^{m+1 / 2}(i+1, j)-k_{5}
\end{aligned}
$$

The iterative procedure is to vary $j$ from $j=1$ to $j=M-1$ in Equation $(G-6)$ in order to obtain new iterates $\phi^{m+1 / 2}(i, j)$ over the entire mesh. Next, vary $i$ from $i=1$ to $i=M-1$ in Equation (G-7) to obtain the new iterates $\phi^{m+1}(i, j)$. Thus, a complete iteration consists first of solving a system of $(M-1)$ simultaneous equations for each of the $(M-1)$ rows of the interion mesh and then of solving a system of $(M-1)$ equations for each of the (M-1) columns of the interior mesh. By alternately sweeping the rows and columns the iteration is continued until successive iterates converge within some desired tolerance over the whole mesh. It may be noted that the existence of the tridiagonal matrix is not only a timesaver but also allows the feasibility of this particular iterative procedure.

The alternating direction method is the only one of the iterations which converged when it was applied to the VTE. This scheme is here and elsewhere particularly recommended for the solution to non-linear dif- 
ferential equations. Both Esch [22] and Pearson [32] use what they call internal smoothing in this iterative technique. The new guess which is calculated from the tridiagonal matrix is modified to get a better guess which is some fraction (they recommend 15 per cent) of the new guess plus some fraction (they recommend 85 per cent) of the old guess. In this study of a vortex ring, successive iterates at a point in space were averaged every second iteration. It is strongly recommended here that a thorough investigation be made to find a systematic method for improving the rate of convergence of this iterative technique which is capable of solving a given non-linear equation. 
APPENDIX H

THE CONVERGENCE STUDY

The standard technique utilized to demonstrate the convergence of a finite difference approximation is to solve the difference equation over a number of successively finer meshes in order to show that the sequence of solutions thus obtained approaches a single result. This result, sometimes called the exact solution, is taken to be the solution to the original differential equation, and the finite difference equation is said to have converged to this answer. However, in a practical problem, the exact solution is not known a priori so successive numerical approximations cannot be compared to it. Consequently, the characteristic which usually determines convergence is the existence of a diminishing difference between successive numerical approximations made over finer and finer meshes. The members of this sequence of solutions really approach only each other. For this reason, in a practical problem convergence is not an absolute state of affairs, and its existence must be qualified by the phrase "within a certain degree of approximation."

It is realized that it would have been desirable to solve both the parabolic and uniform profile cases over a series of mesh sizes and time step increments. Unfortunately, the long computer time necessary to solve just one of these problems made this approach infeasible. For example, to solve the parabolic profile case for $M=24$ 
(529 interior mesh points) over the time period for which the impulsive velocity of Figure 5 was non-zero took from eight to ten hours of machine time on the Burrough's B-5500 computer. Consequently, only two different spatial meshes were employed in each of the cases studied. Since a series of solutions could not be compared for each case, it is the purpose of this convergence study to demonstrate that the two solutions give approximately the same results.

\section{The Parabolic Profile}

The parameters used in the two solutions for the parabolic profile are outlined in Table $\mathrm{H}-1$.

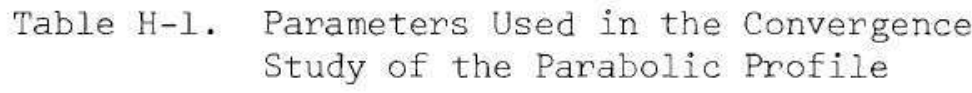

\begin{tabular}{|c|c|c|c|c|c|}
\hline $\begin{array}{l}\text { Problem } \\
\text { Number }\end{array}$ & M & $\delta t$ & P & a & b \\
\hline I & 16 & 1.0 & 8 & 0.69315 & 0.69315 \\
\hline II & 24 & 0.5 & 12 & 0.69315 & 0.69315 \\
\hline \multicolumn{3}{|c|}{$R=0.04 \mathrm{ft}}$. & \multicolumn{3}{|c|}{$\mathrm{U}=2 \mathrm{ft} / \mathrm{sec}$} \\
\hline \multicolumn{2}{|c|}{ Rey $=533$} & & \multicolumn{3}{|c|}{$\begin{array}{l}\text { Tolerance of } \\
\text { Iteration }\end{array}$} \\
\hline
\end{tabular}

Since the dependent variables of the problems are the vorticity and stream function distributions, these are shown in Figures $\mathrm{H}-1$ through $\mathrm{H}-10$ for the times $t=2,6,10,14$ and 18 . In order to present another point of view, the vorticity, stream function, and 
axial and radial velocity components for the point $z=1$ and $r=1$ are presented as functions of time in Figures H-ll through H-14. Finally, the boundary value of vorticity at the edge of the exit plane is given in Figure $\mathrm{H}-15$ as a function of time.

Inspection of Figures $\mathrm{H}-\mathrm{I}$ through $\mathrm{H}-10$ shows that the vorticity distributions compare well over the time period investigated, and the streamlines show excellent agreement. It is to be expected that the comparison of the stream function patterns should be somewhat better than that of the vorticity distributions. The vorticity is a rather complicated combination of the first and second derivatives of the stream function, and any error in the stream function will be magnified in its derivatives.

A better quantitative evaluation of the method may be obtained from the study of Figures $\mathrm{H}-\mathrm{Il}$ through $\mathrm{H}-14$. The maximum error in the vorticity and the axial velocity is about 10 per cent for medium to large absolute values of each variable. The radial velocity offers a better comparison with an error of about 7 per cent, and for the stream function, the values calculated on the two different grids cannot be distinguished from each other in Figure $\mathrm{H}-12$.

Finally, Figure H-15 illustrates the boundary vorticity at $r=1$ as a function of time. This plot is noteworthy for several reasons. First, it demonstrates that the method employed in evaluating the boundary value of vorticity is reliable; the agreement between the two cases is good. Second, a comparison of Figure $\mathrm{H}-15$ and Figure $\mathrm{H}-11$ shows that a discrepancy between the vorticities of the two cases on the 


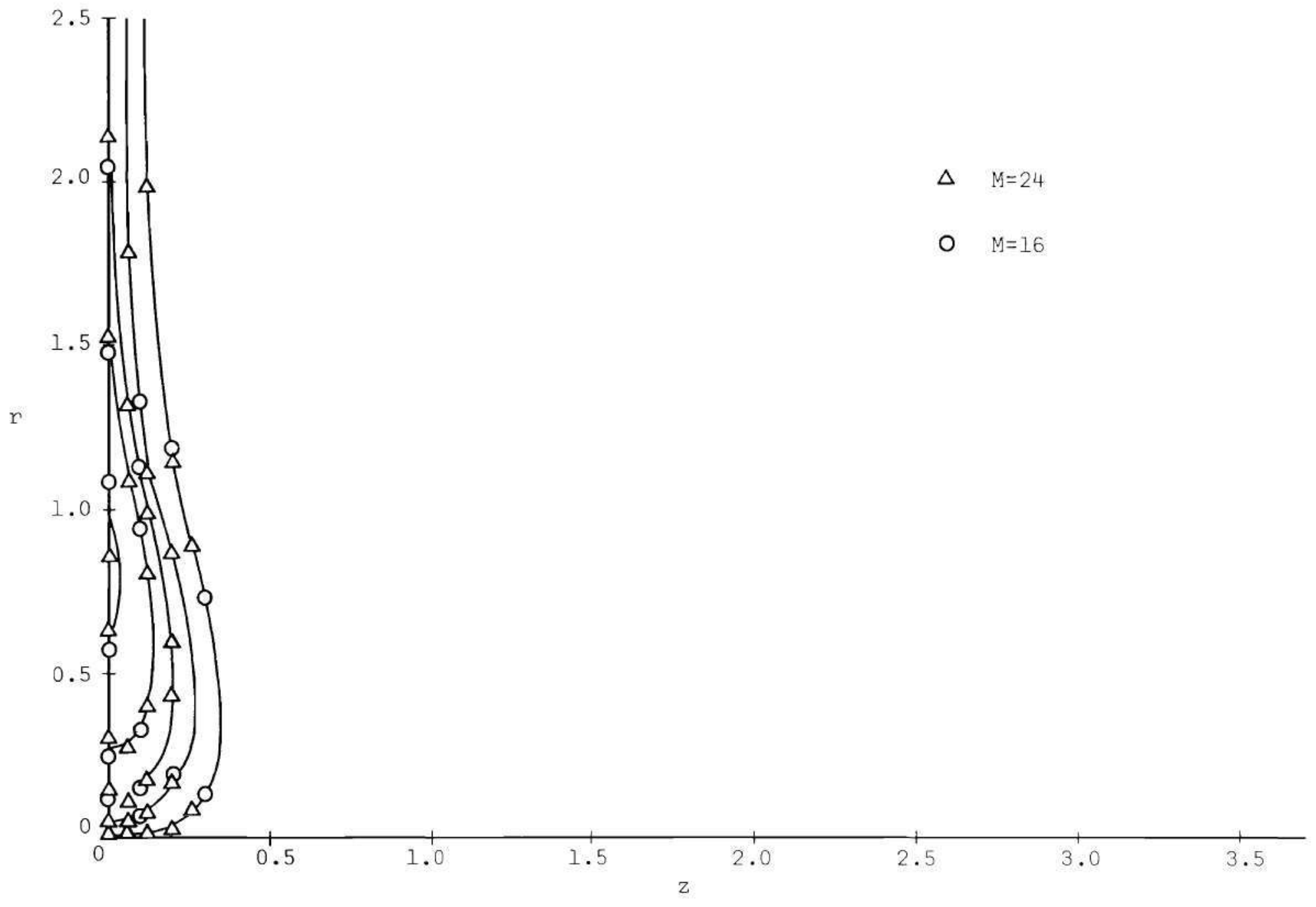

Figure $\mathrm{H}-1$. Vorticity Distribution at $t=2--$ Parabolic Profile 


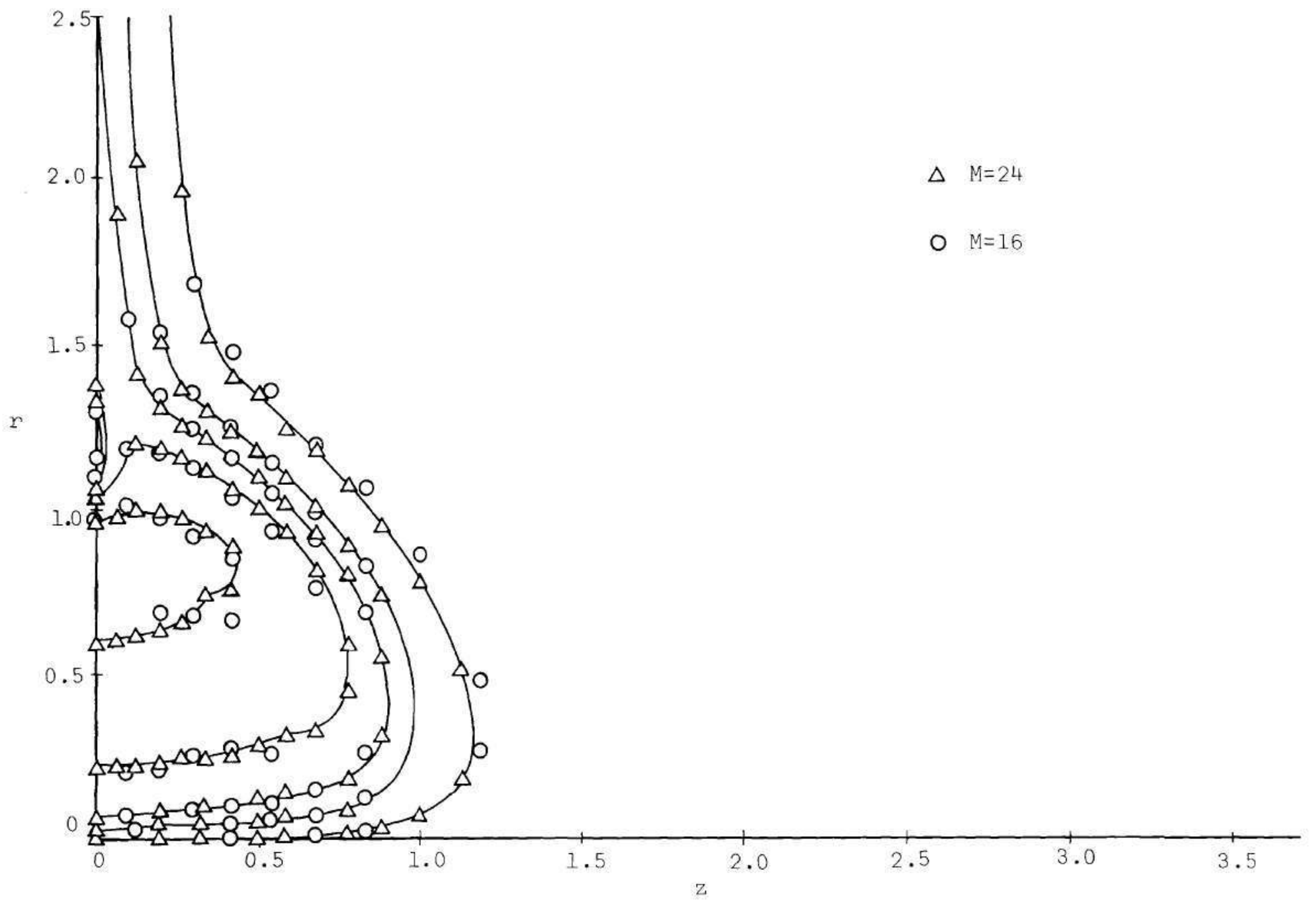

Figure H-2. Vorticity Distribution at $t=6--$ Parabolic Profile 


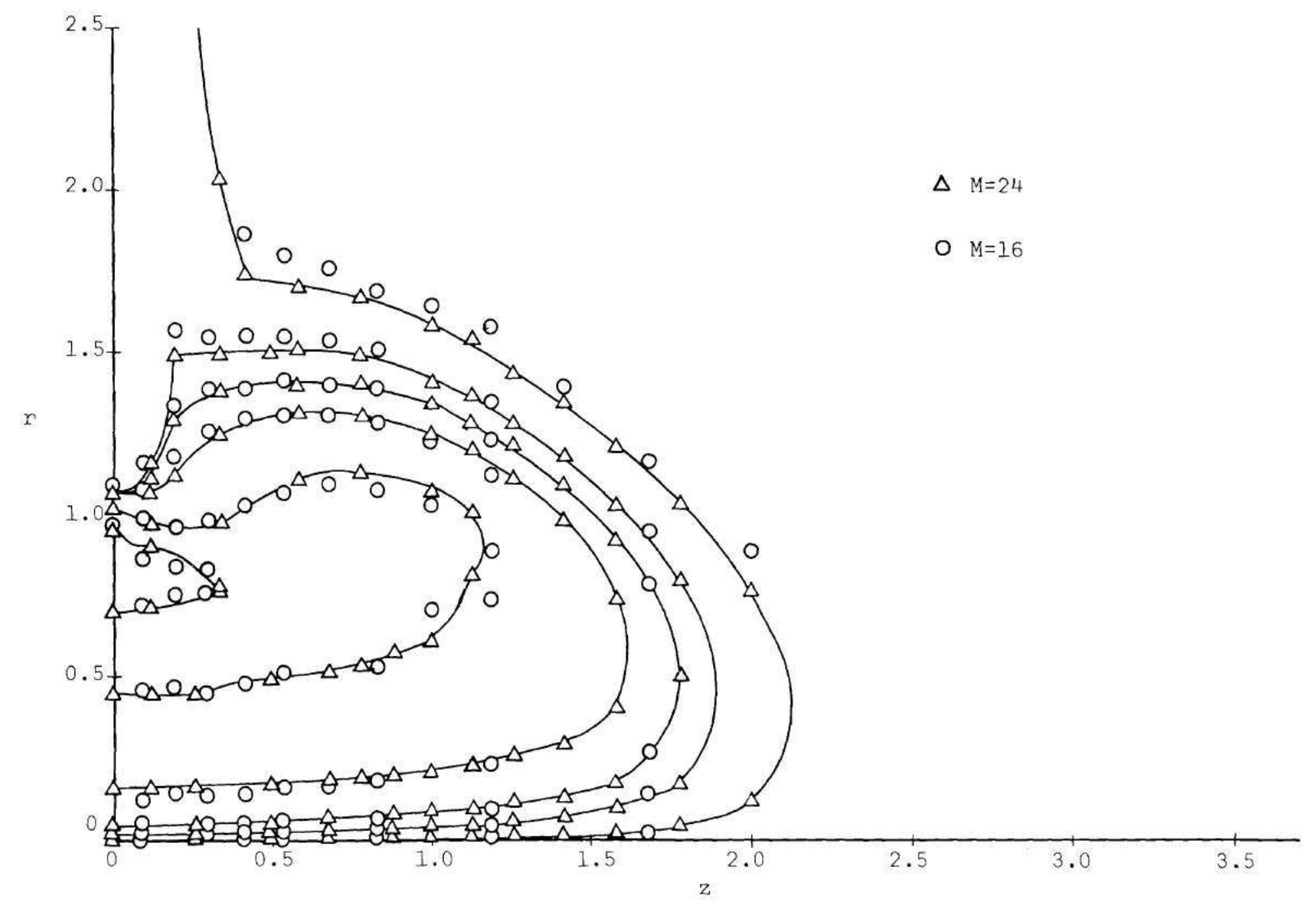

Figure H-3. Vorticity Distribution at $t=10--$ Parabolic Profile 


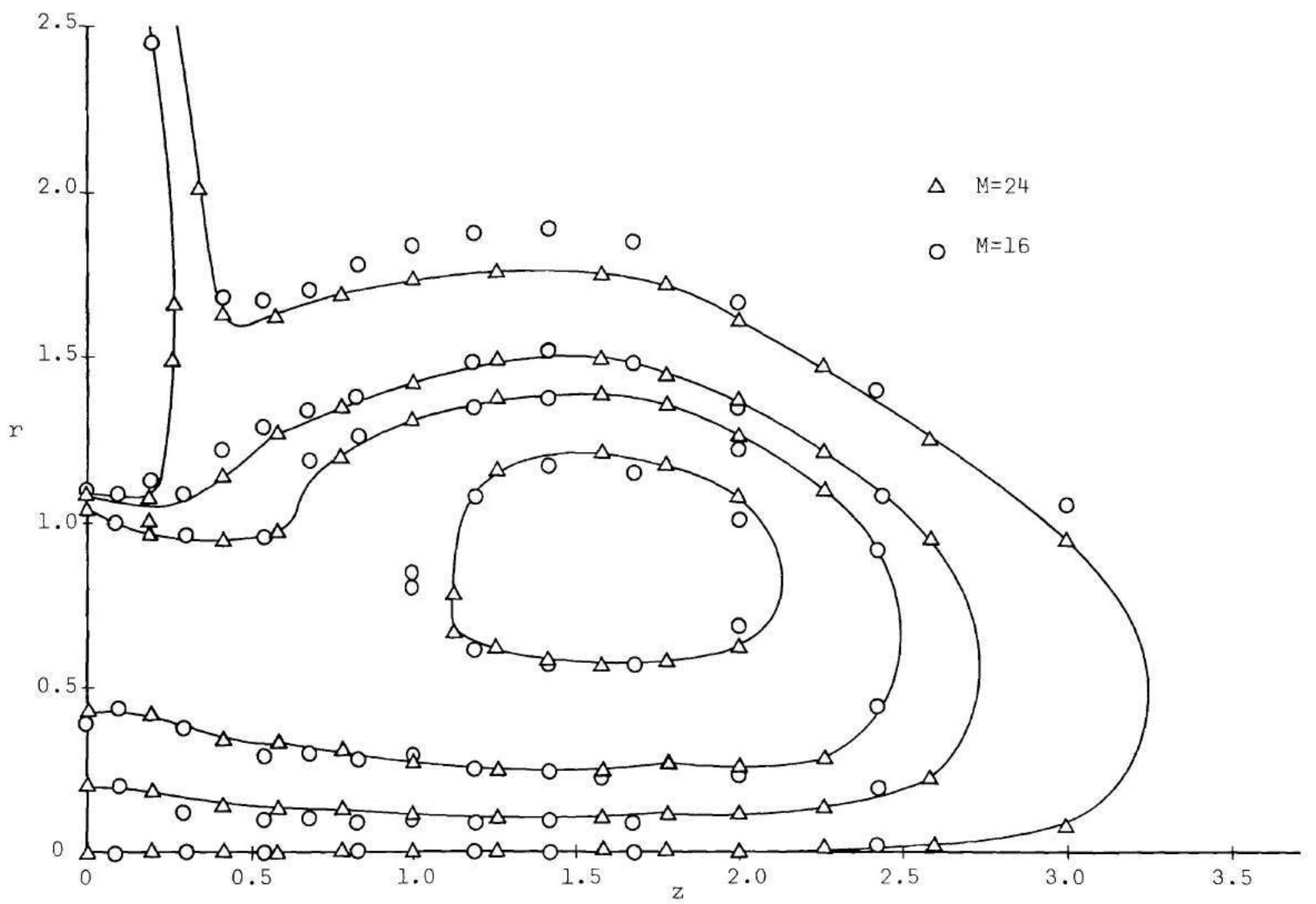

Figure H-4. Vorticity Distribution at $t=14 \ldots$ Parabolic Profile 


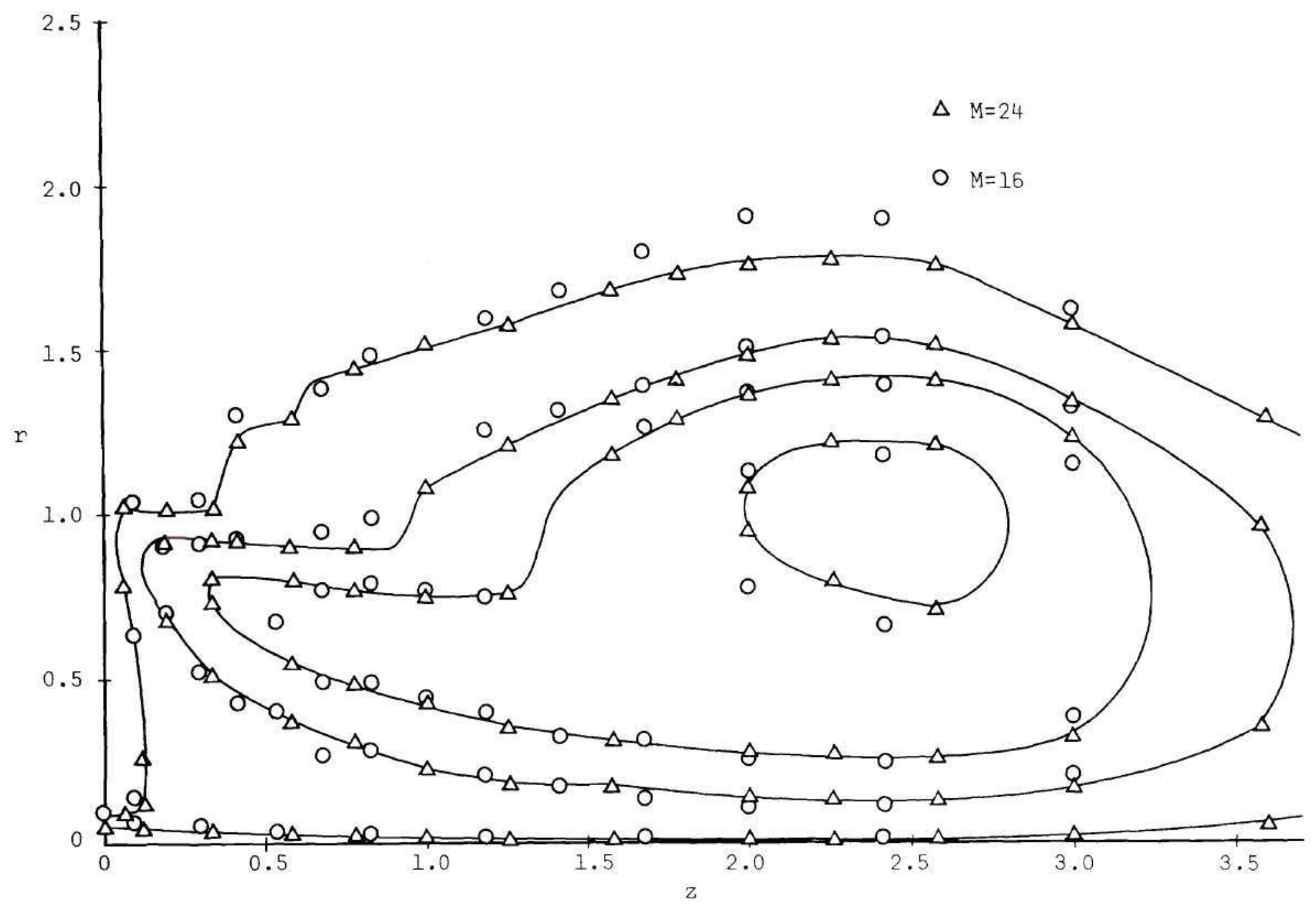

Figure H-5. Vorticity Distribution at $t=18$-- Parabolic Profile 


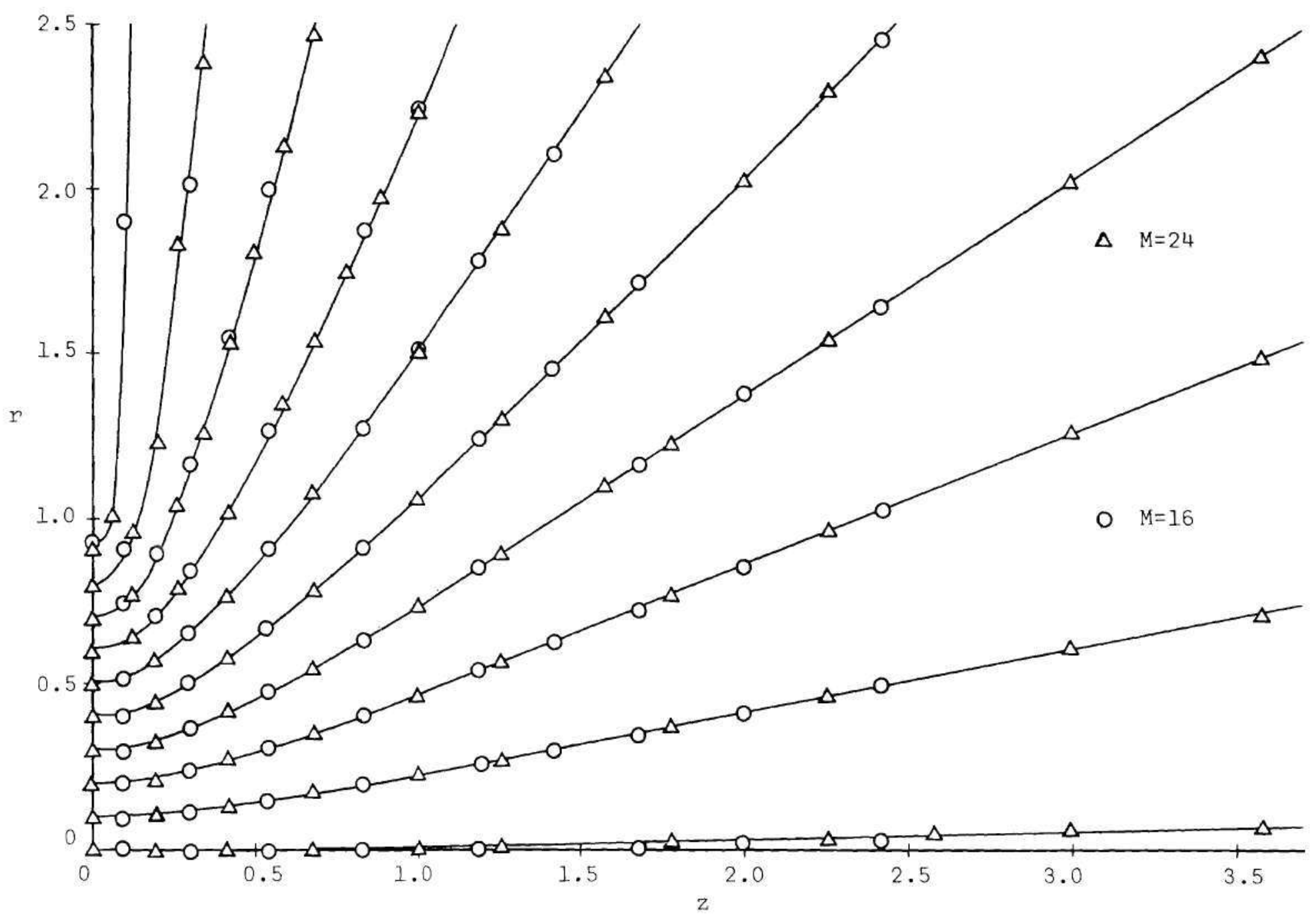

Figure H-6. Streamline Pattern at $t=2$-- Parabolic Profile 


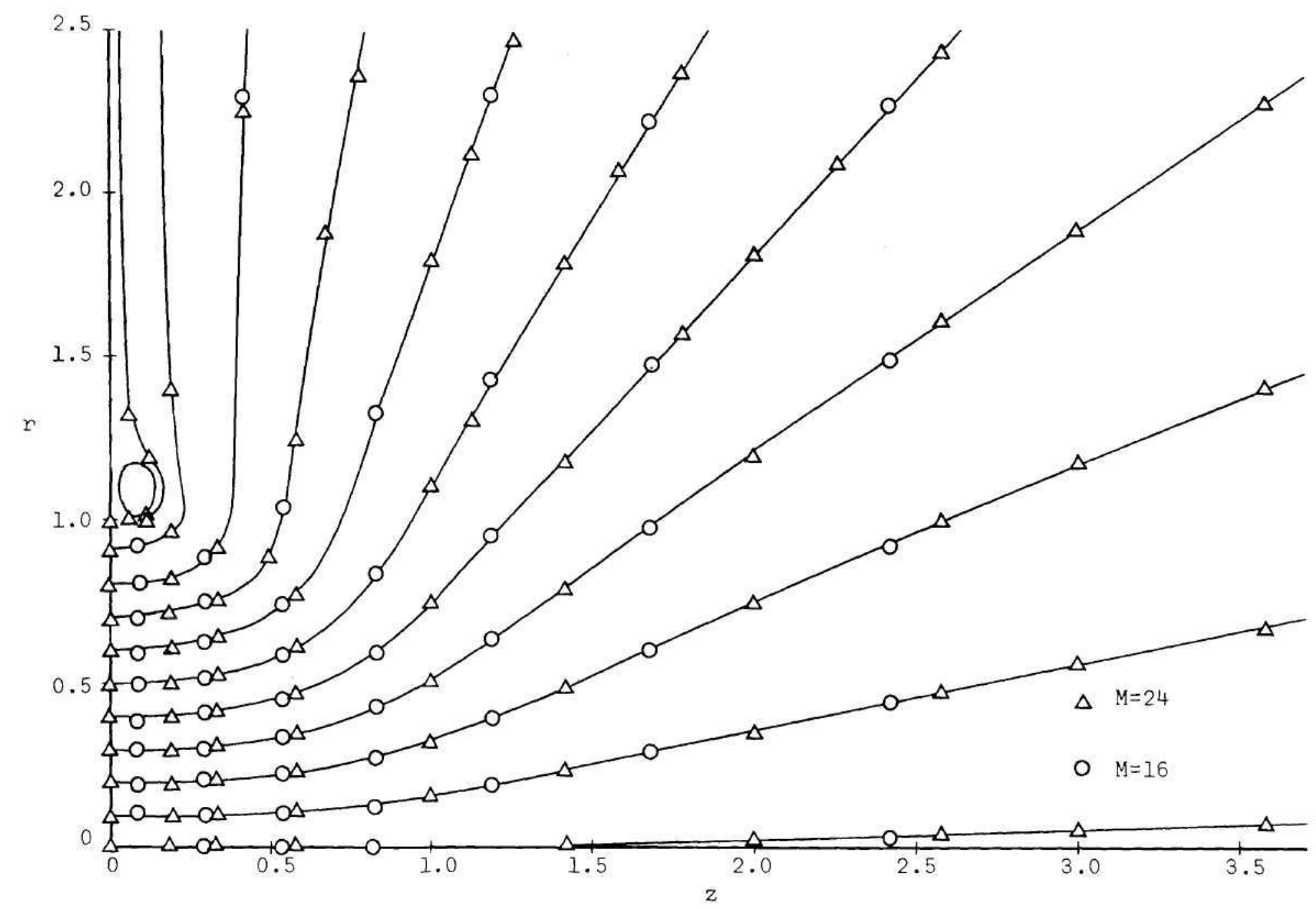

Figure H-7. Streamline Pattern at $t=6$-- Parabolic Profile 


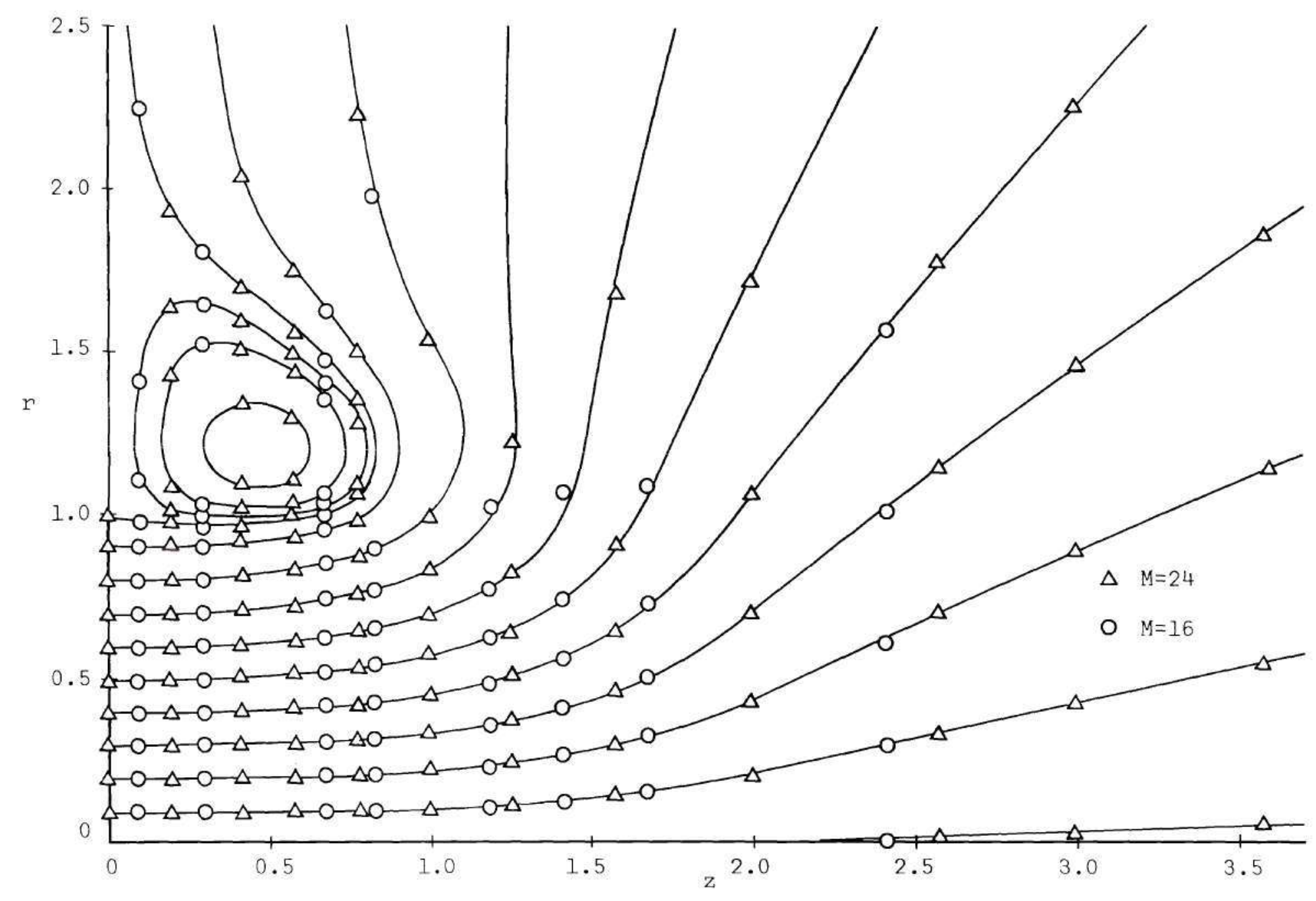

Figure H-8. Streamline Pattern at $t=10$-- Parabolic Profile 


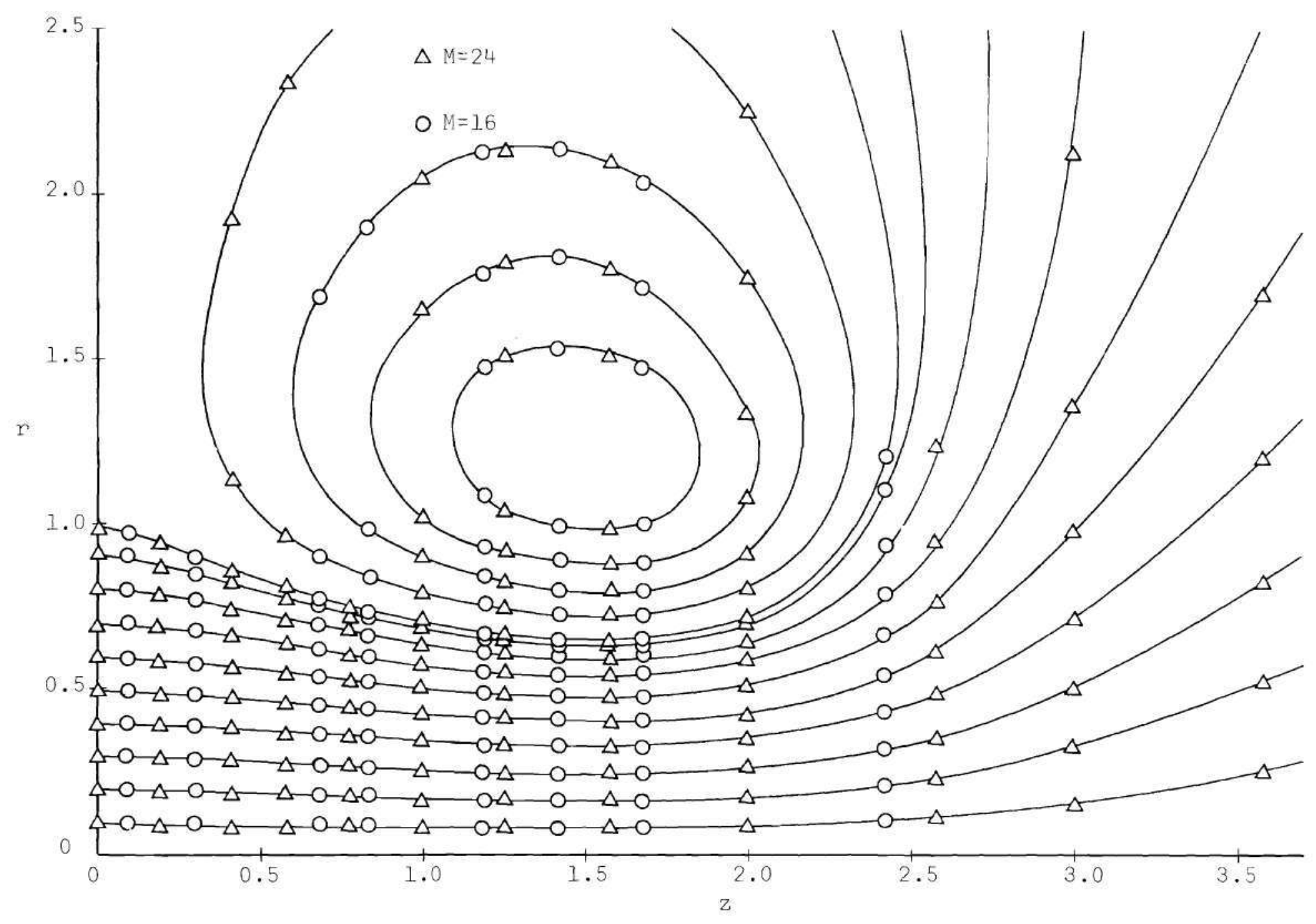

Figure H-9. Streamline Pattern at $t=14$-- Parabolic Profile 


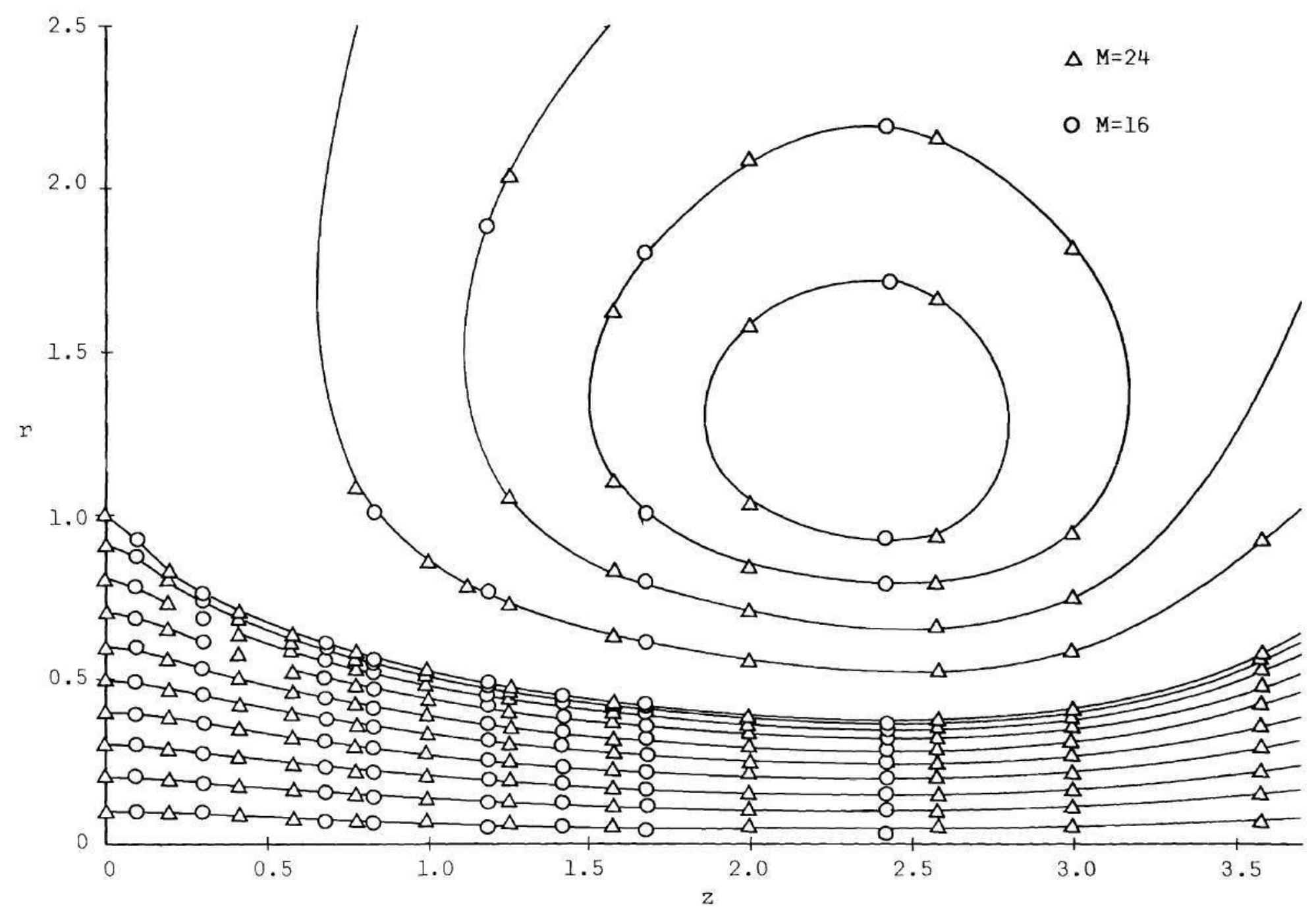

Figure H-10. Streamline Pattern at $t=18$-- Parabolic Profile 


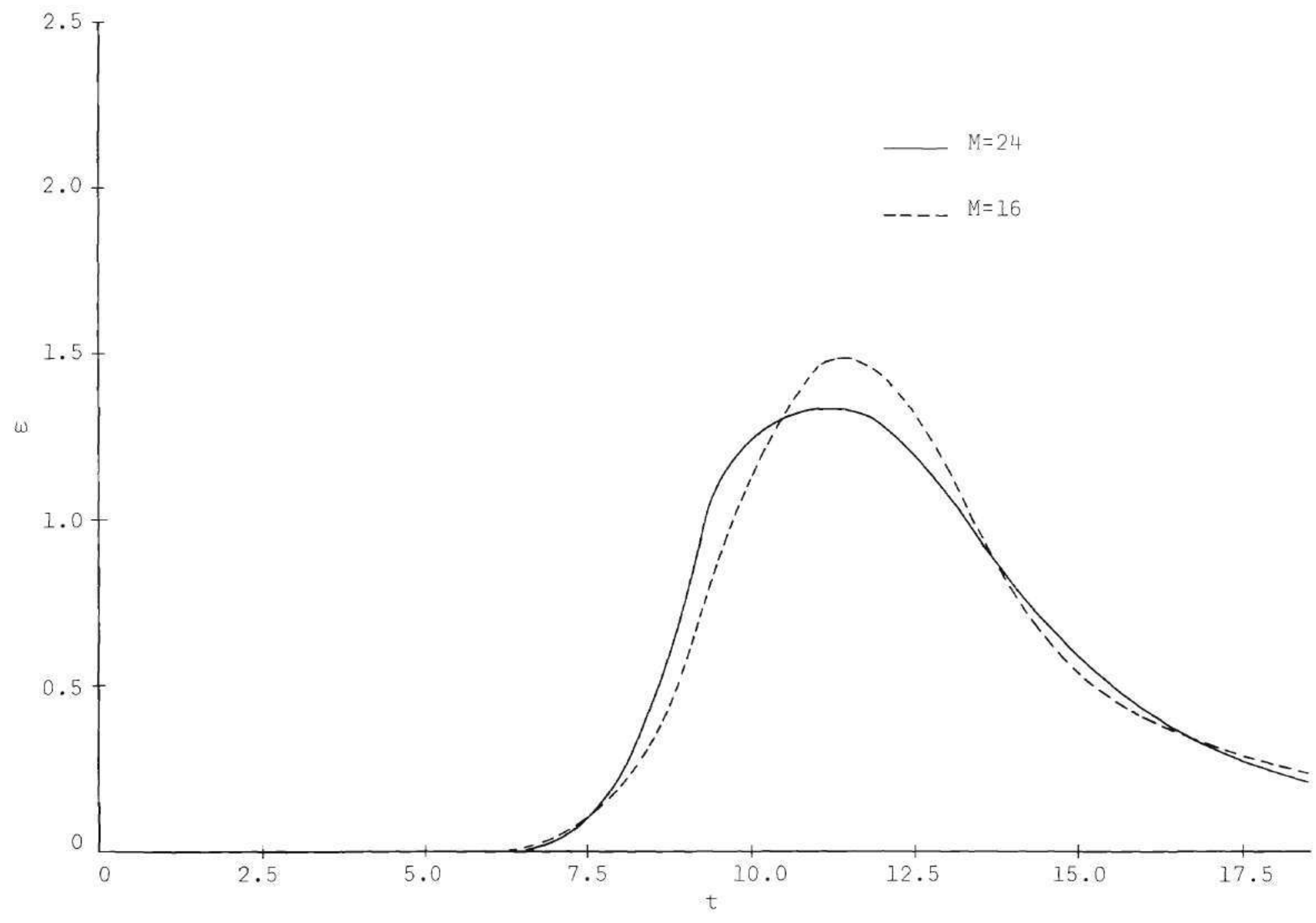

Figure H-1l. Vorticity at $z=1, r=1$-. Parabolic Profile 


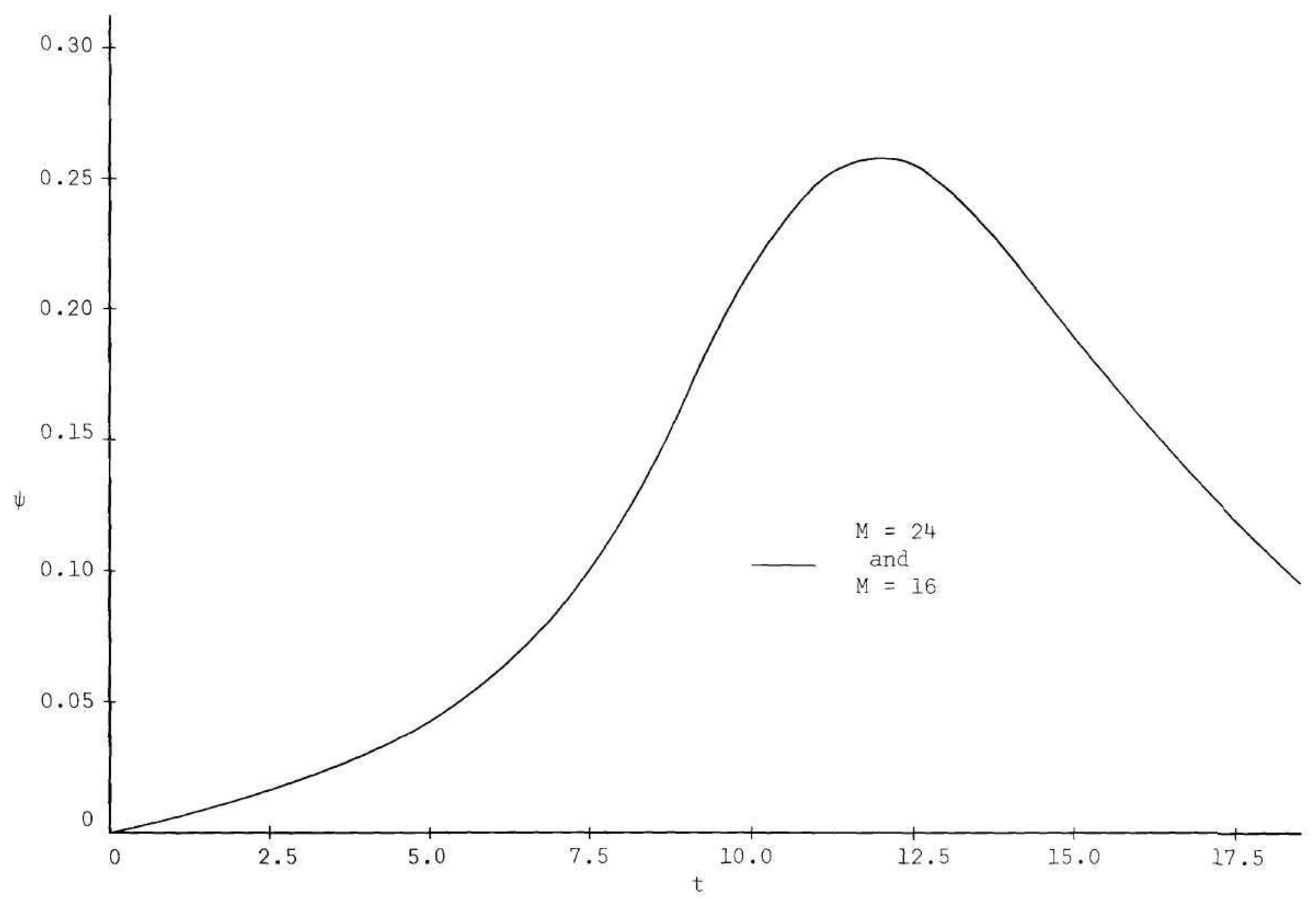

Figure H-12. Stream Function at $z=1, r=1--$ Parabolic Profile 


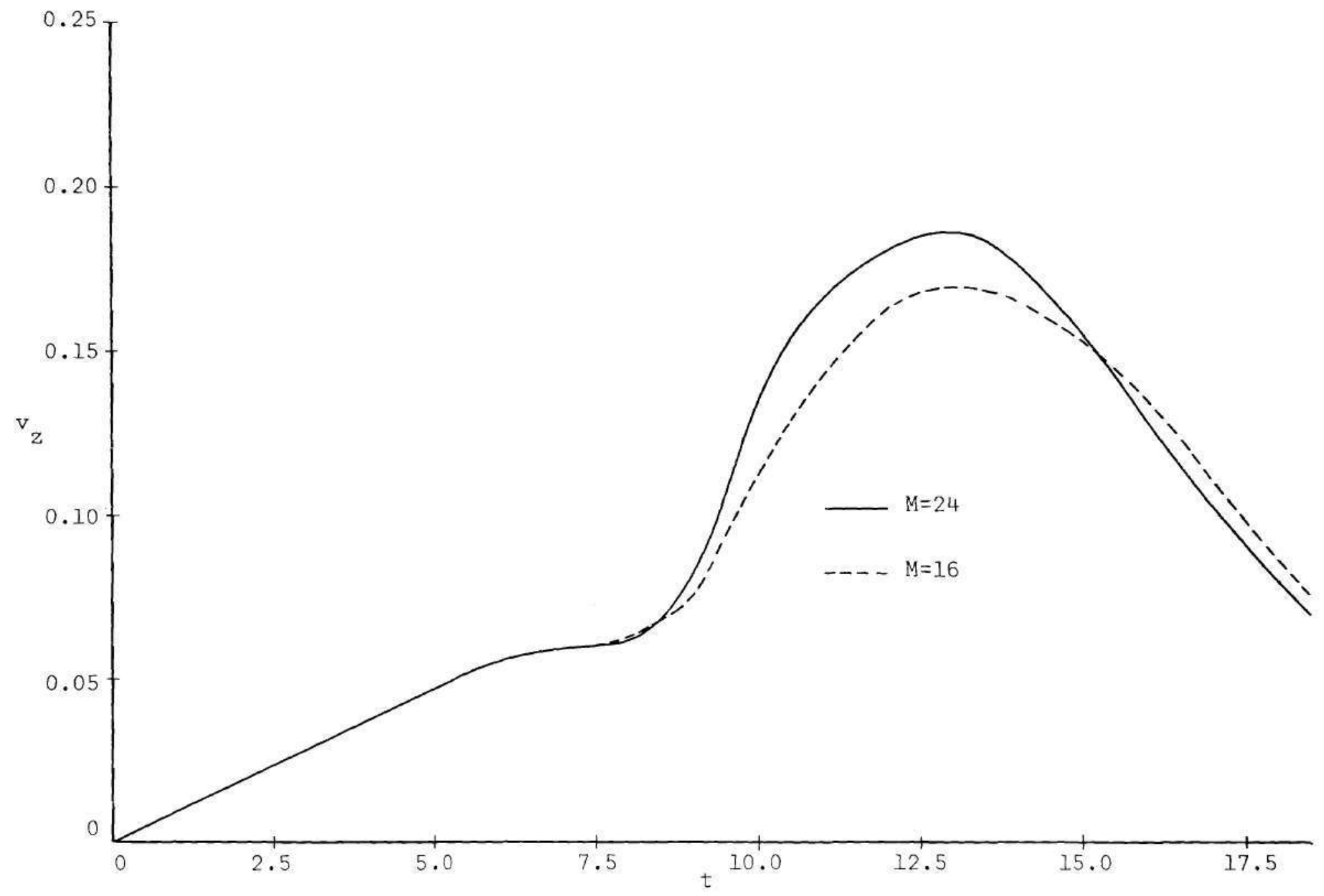

Figure H-13. Axial Velocity at $z=1, r=1$-- Parabolic Profile 


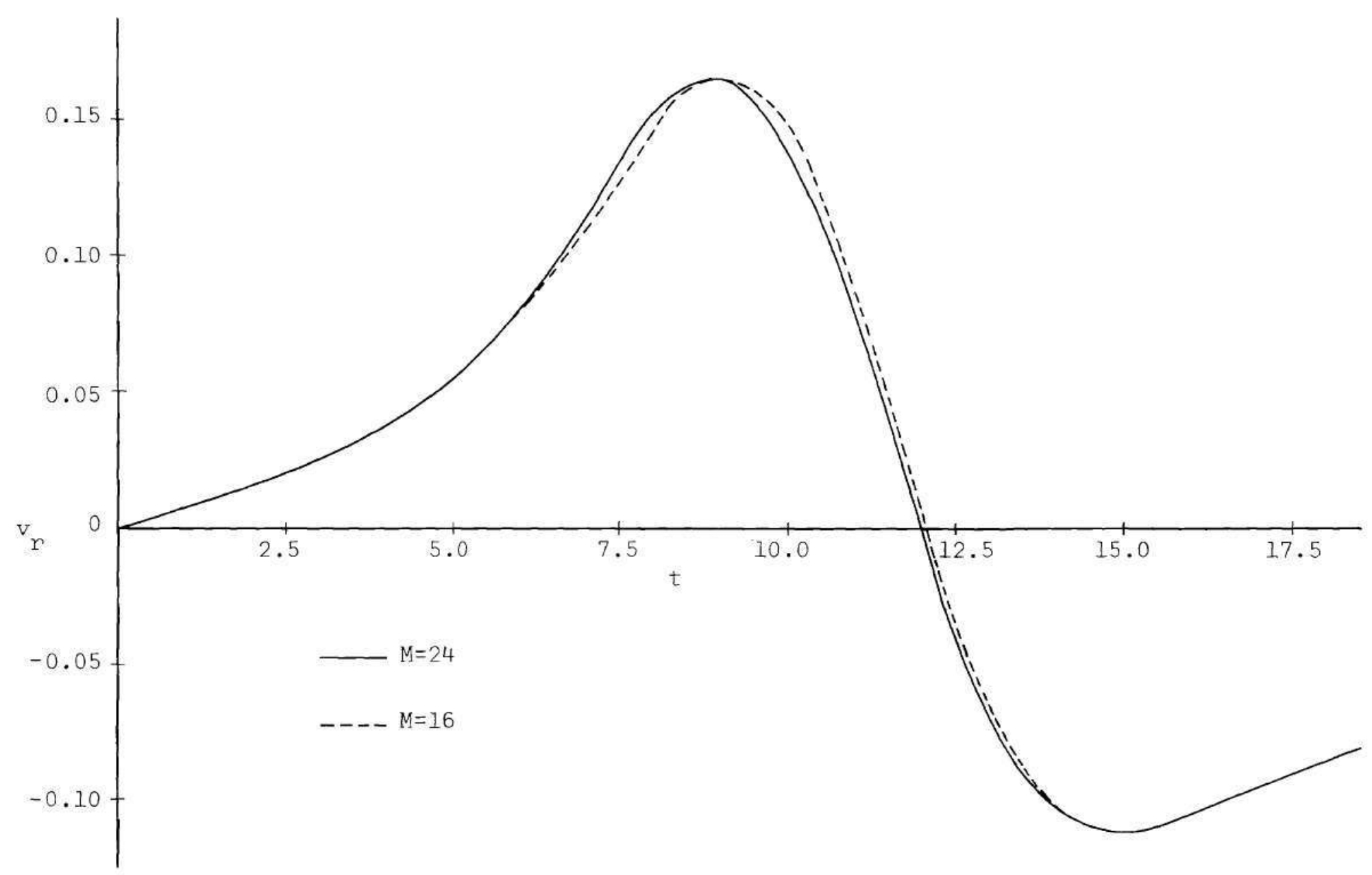

Figure H-14. Radial Velocity at $z=1, r=1$-- Parabolic Profile 


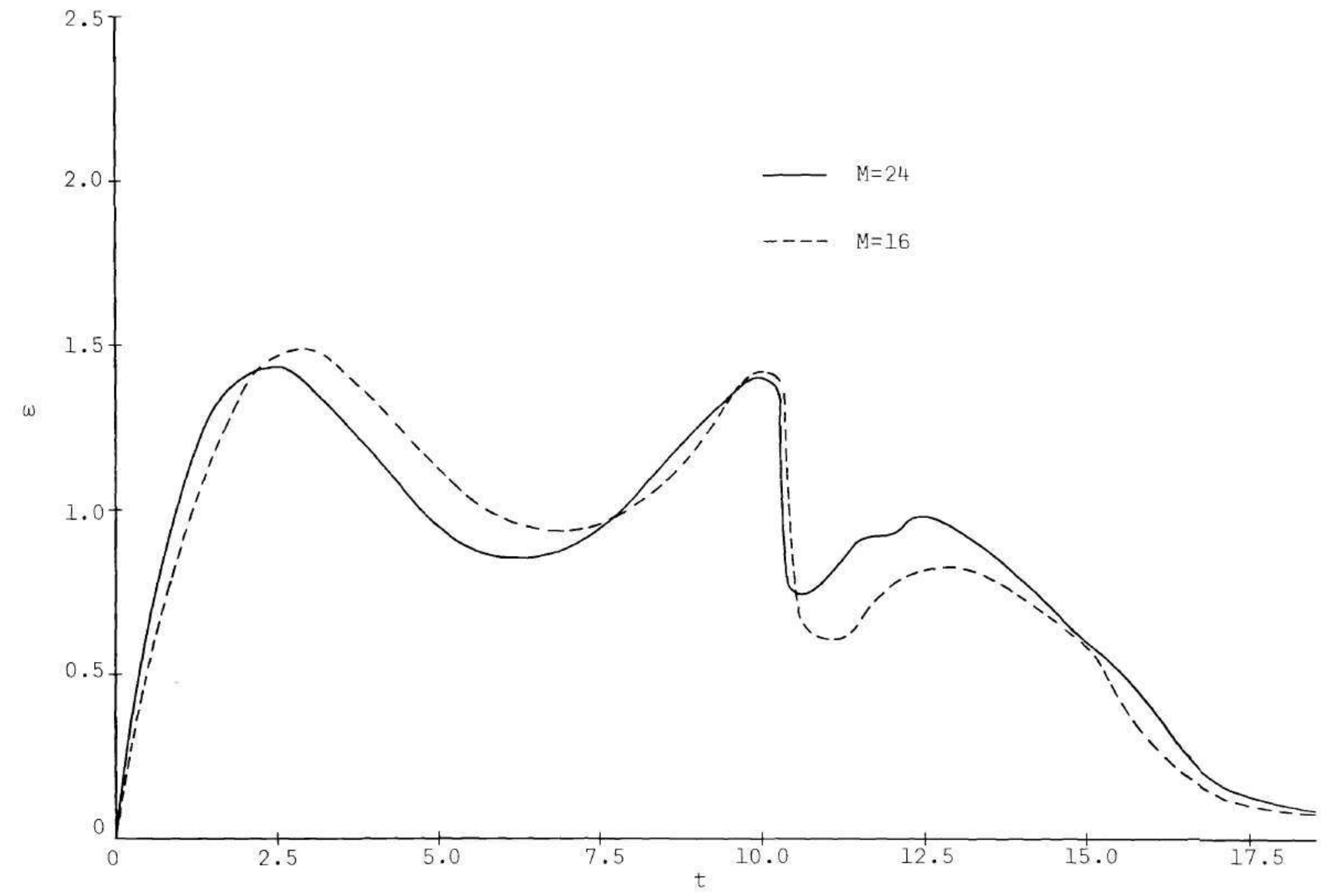

Figure H-15. Vorticity at $z=0, r=1$-- Parabolic Profile 
boundary does not increase in the interior of the flow field. The difference between the curves in Figure $\mathrm{H}-1 \mathrm{l}$ is no greater than that between the curves of Figure $\mathrm{H}-15$.

\section{The Uniform Profile}

The parameters used in the two solutions for the uniform profile are outlined in Table $\mathrm{H}-2$.

Table $\mathrm{H}-2$. Parameters Used in the Convergence Study of the Uniform Profile

\begin{tabular}{|c|c|c|c|c|c|}
\hline $\begin{array}{l}\text { Problem } \\
\text { Number }\end{array}$ & M & $\delta t$ & $\mathrm{P}$ & a & $\mathrm{b}$ \\
\hline I & 16 & 0.5 & 8 & 0.69315 & 0.69315 \\
\hline II & 24 & 0.5 & 12 & 0.69315 & 0.69315 \\
\hline \multicolumn{3}{|c|}{$R=0.04 \mathrm{ft}}$. & \multicolumn{3}{|c|}{$\mathrm{U}=2 \mathrm{ft} / \mathrm{sec}$} \\
\hline \multicolumn{2}{|l|}{ Rey $=533$} & & \multicolumn{3}{|c|}{ Tolerance of } \\
\hline
\end{tabular}

Typical values of the dependent variables, the vorticity and the stream function, are plotted in the $z, r$ plane in Figures $\mathrm{H}-16$ through $\mathrm{H}-25$ for the times $t=2,6,10,14$, and 18. These figures demonstrate qualitative agreement between the two cases. In order to present data amenable to quantitative comparison, the vorticity, the stream function, and the axial and radial velocity components at the point $z=1$ and $r=1$ are shown as functions of time in Figures $\mathrm{H}-26$ through $\mathrm{H}-29$. Finally, the boundary vorticity at $r=1$ is presented as a function 


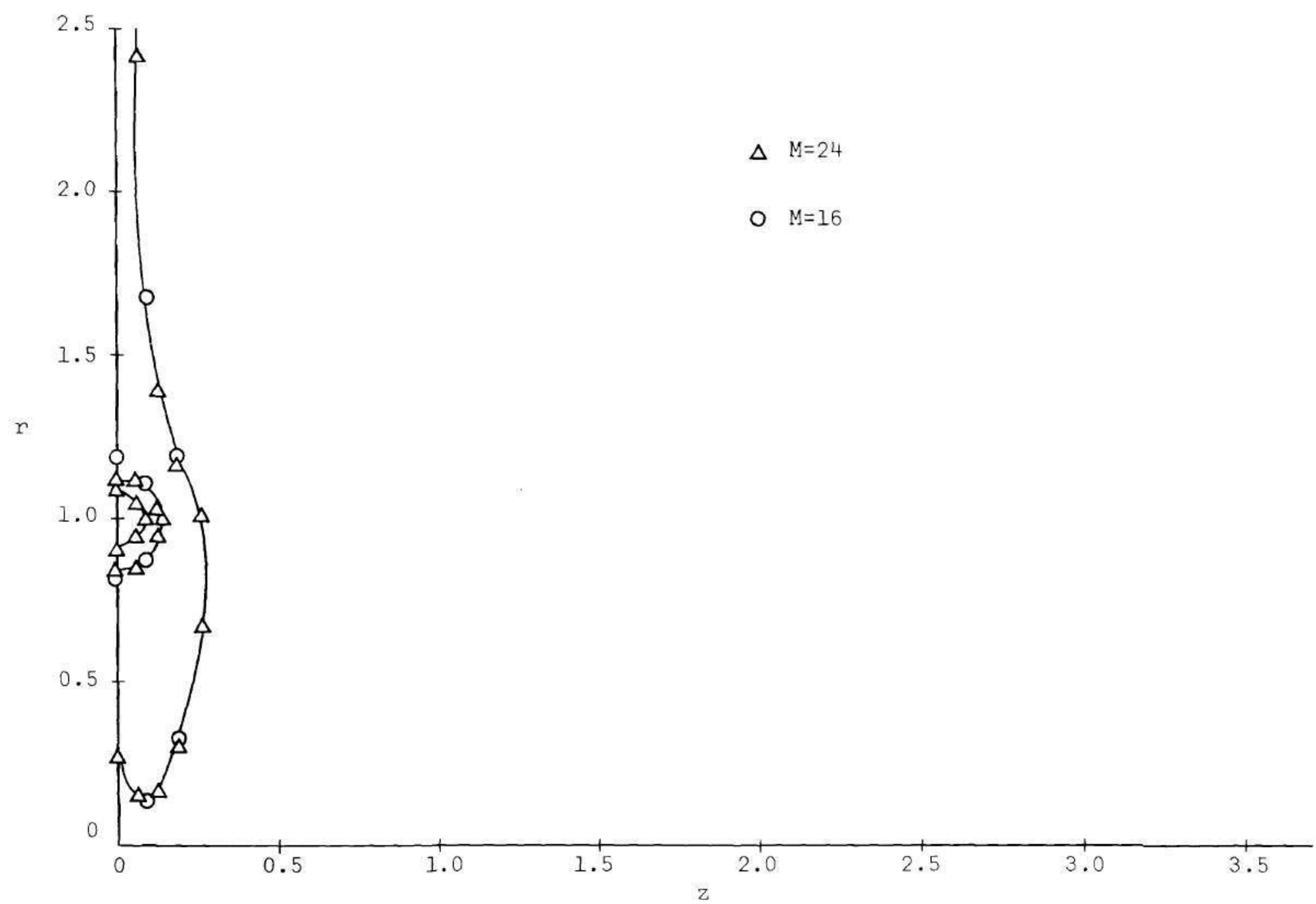

Figure H-16. Vorticity Distribution at $t=2--$ Uniform Profile 


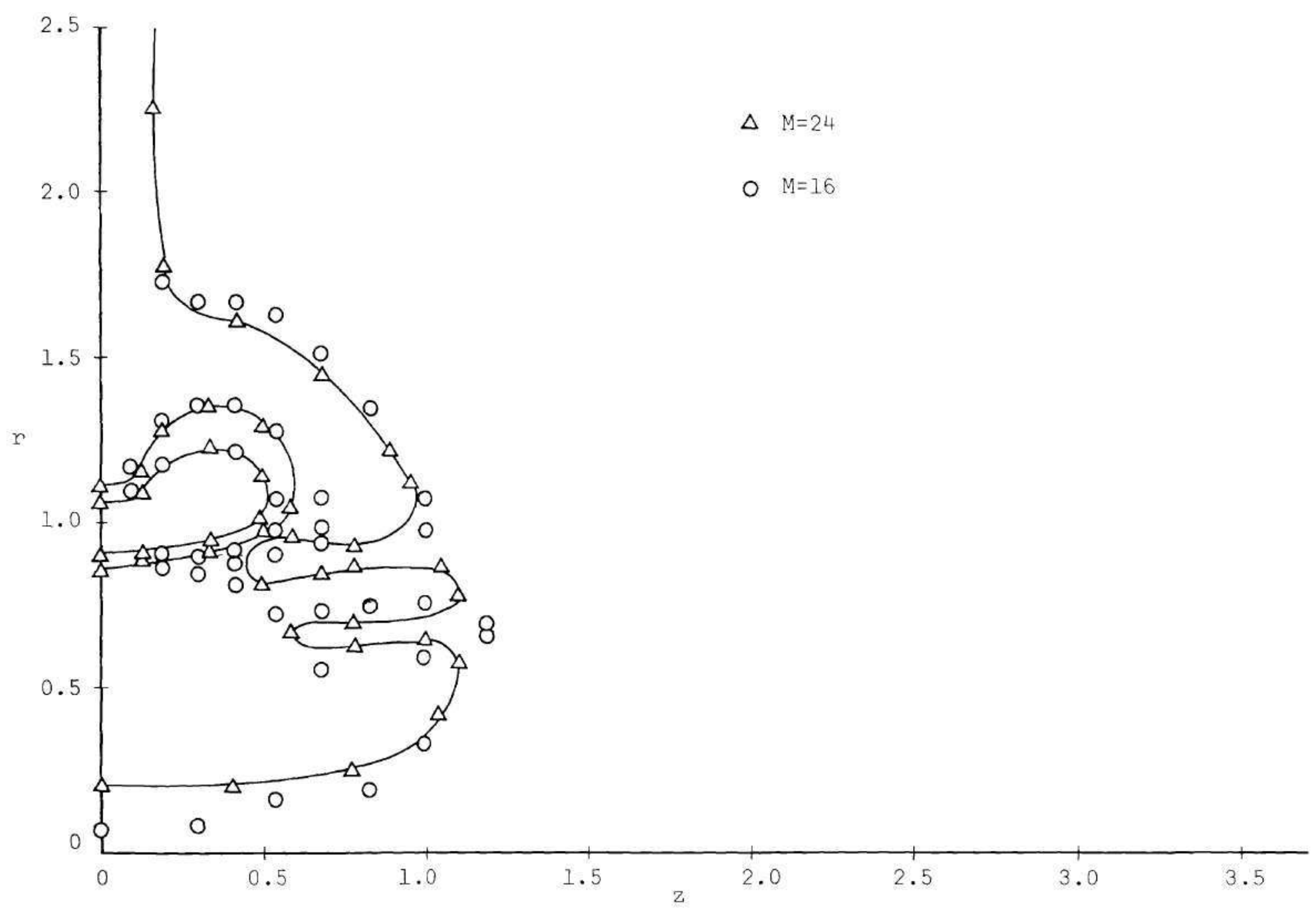

Figure H-17. Vorticity Distribution at $t=6$-- Uniform Profile 


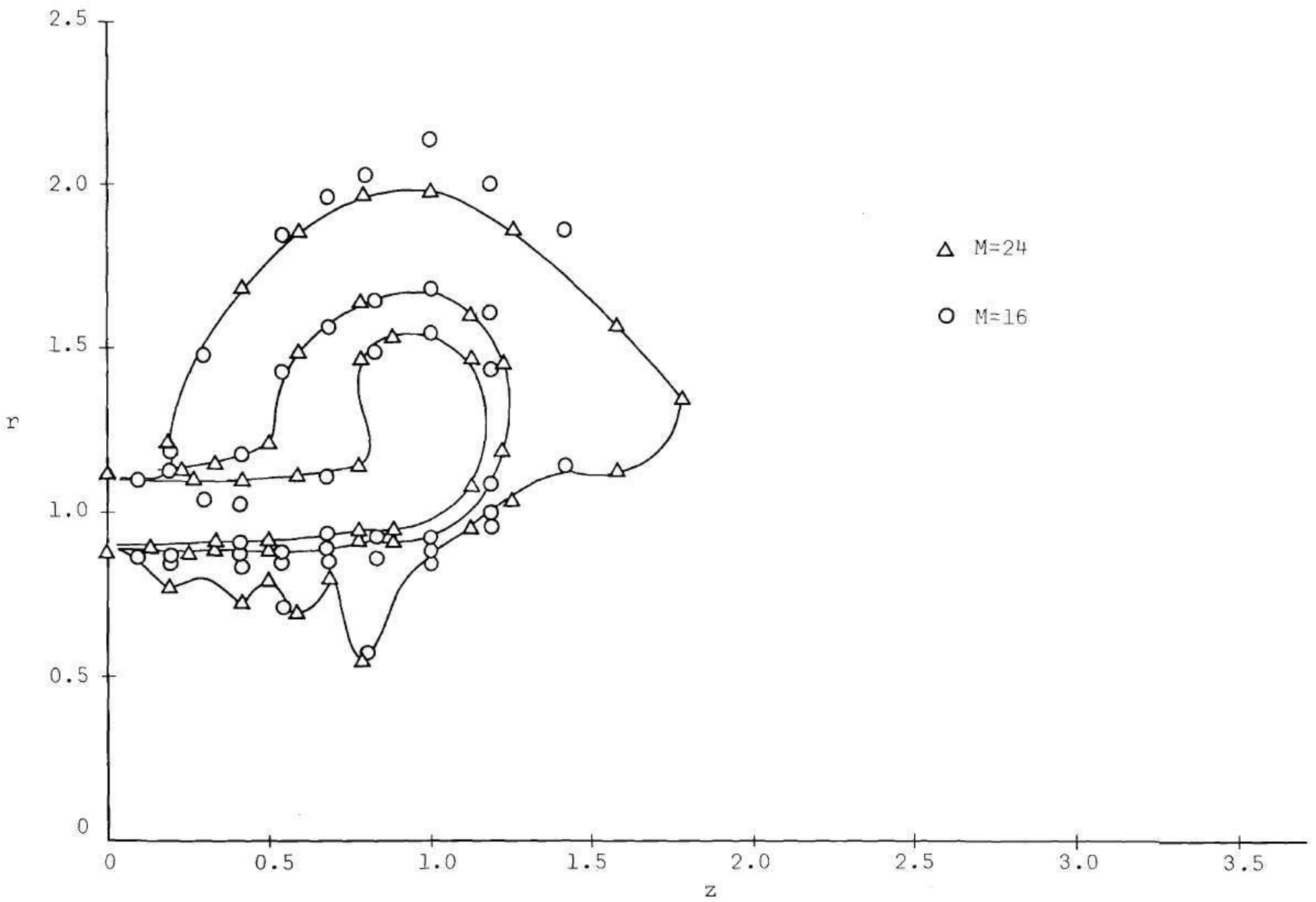

Figure H-18. Vorticity Distribution at $t=10$-- Uniform Profile 


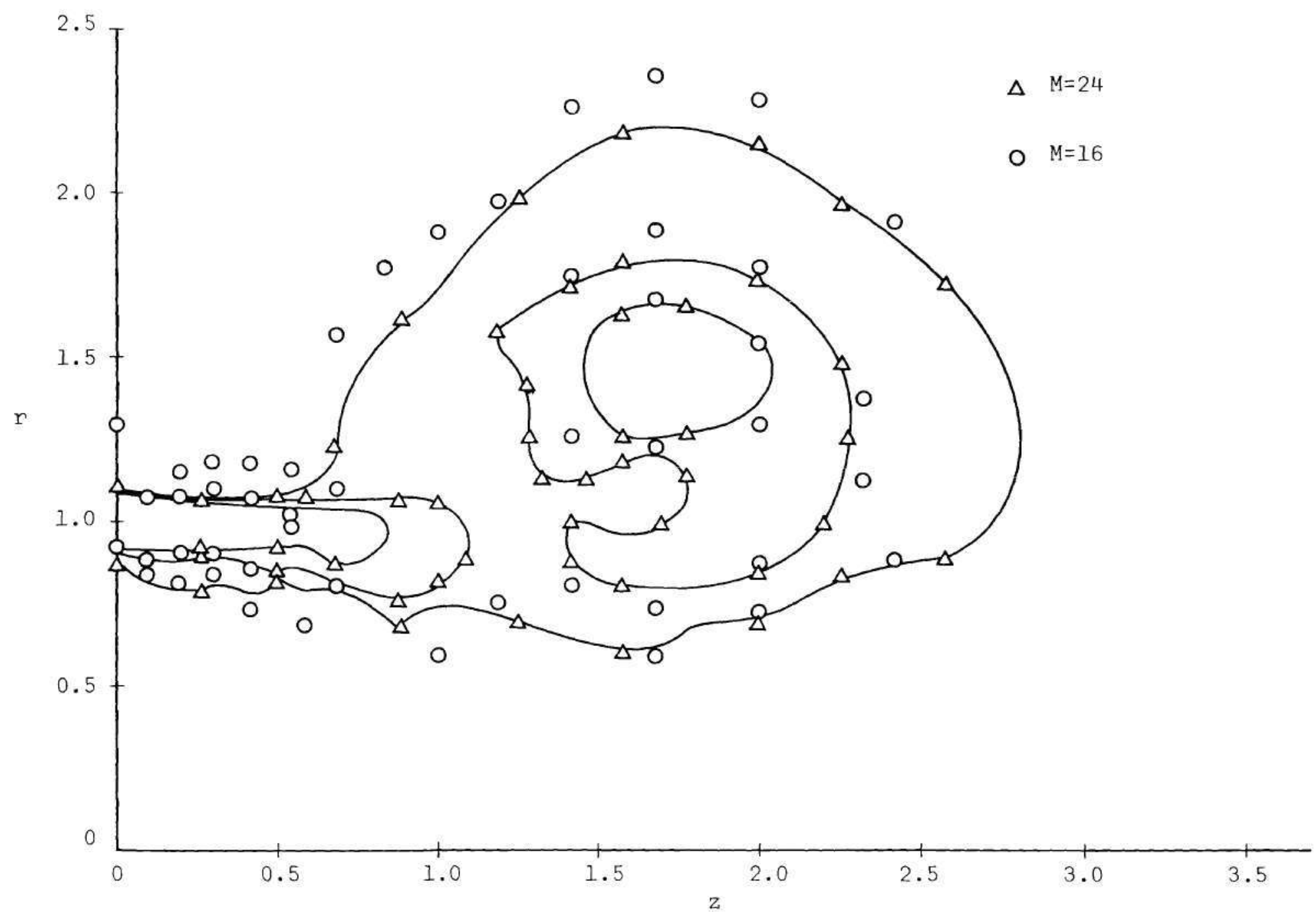

Figure H-19. Vorticity Distribution at $t=14$-- Uniform Profile 


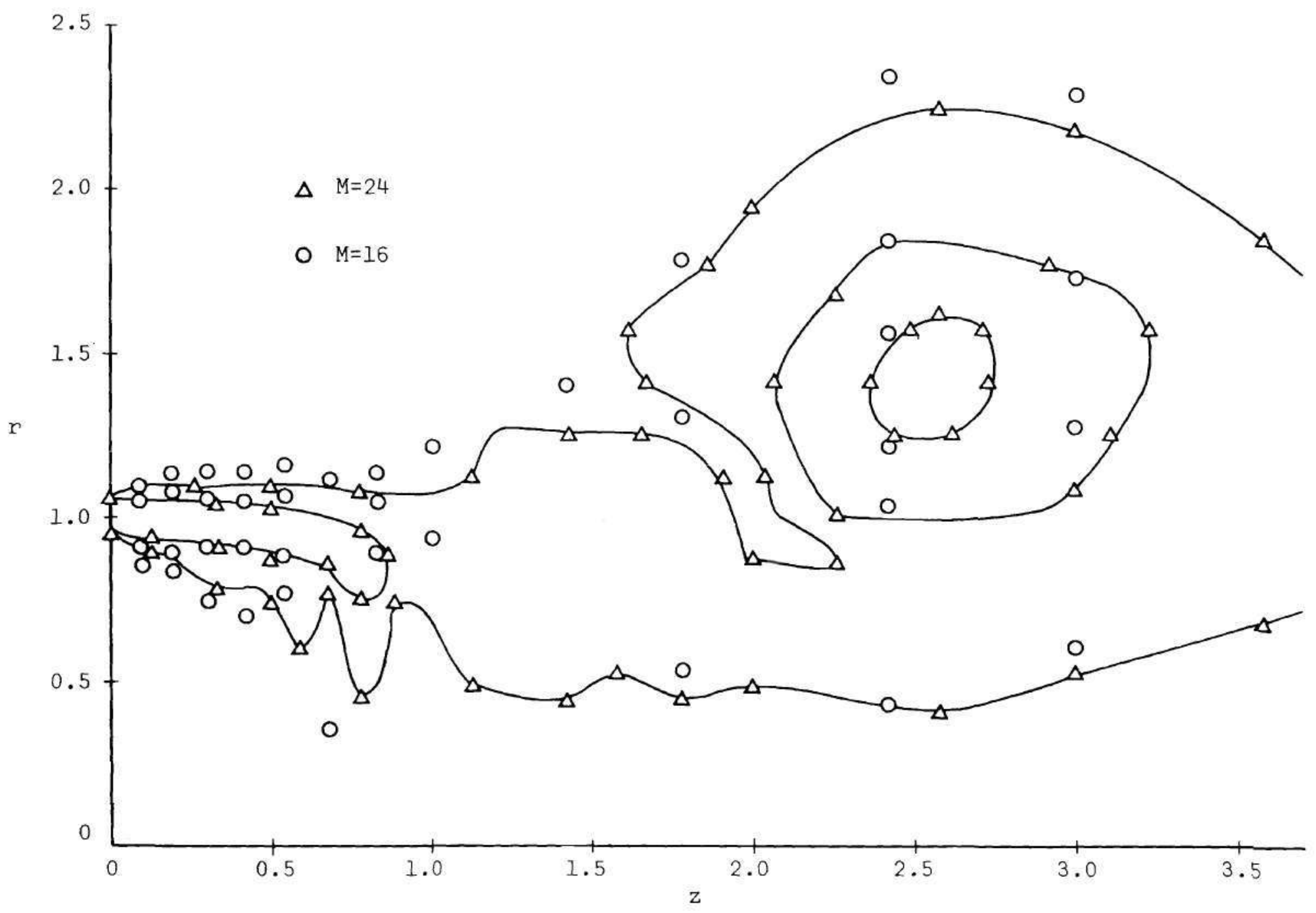

Figure H-20. Vorticity Distribution at $t=18$-- Uniform Profile 


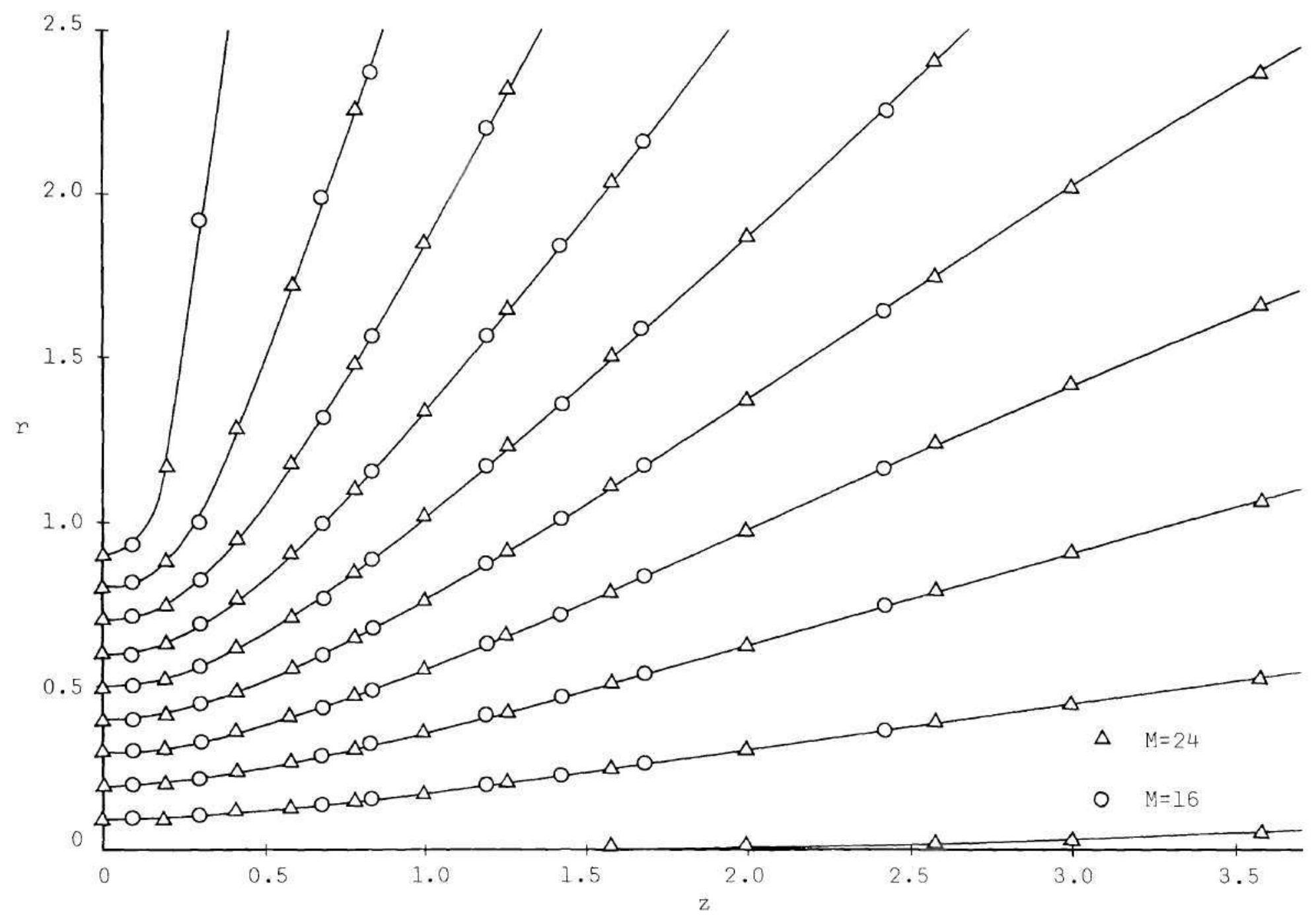

Eigure H-2I. Streamline Pattern at $t=2$-- Uniform Profile 


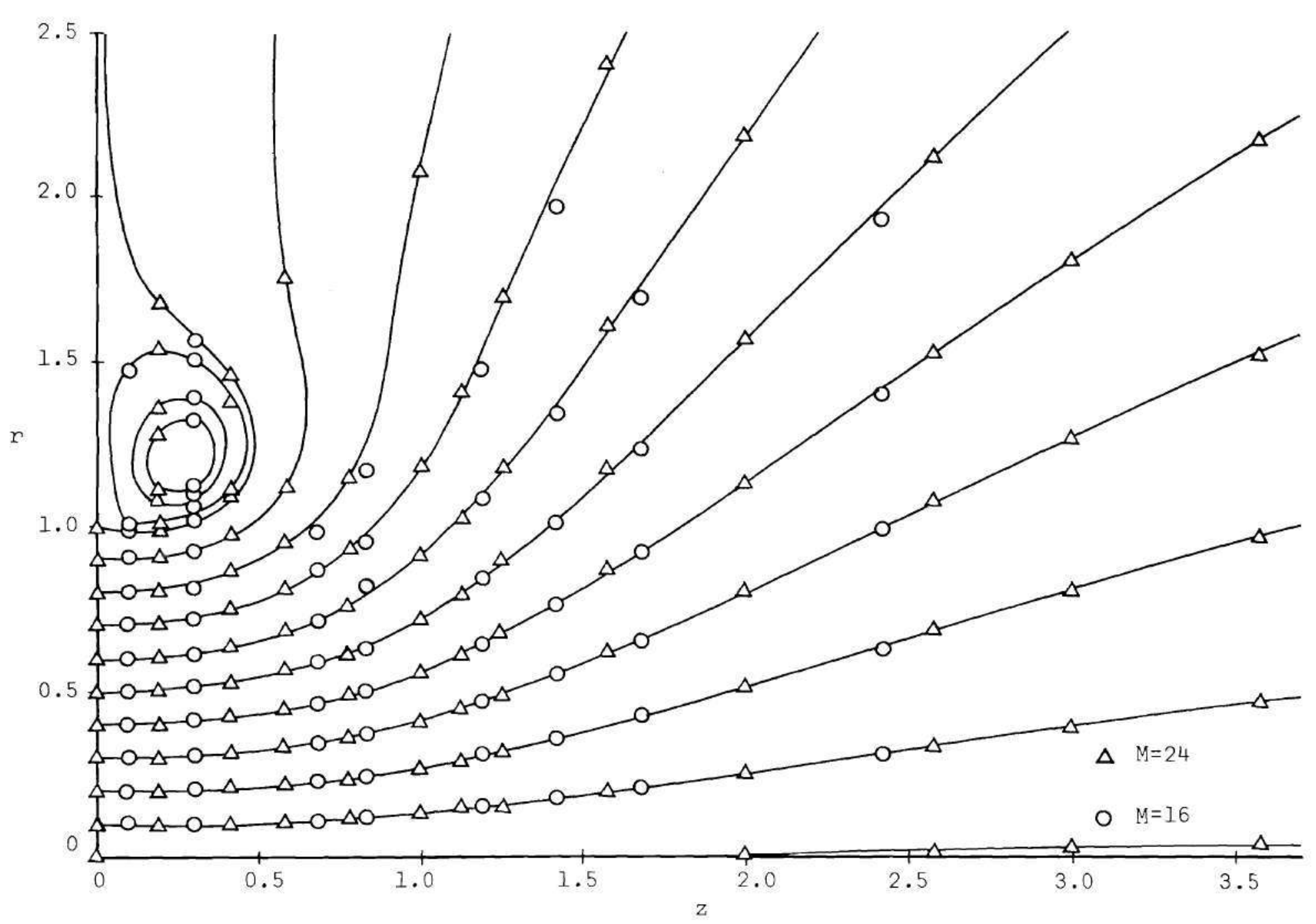

Figure H-22. Streamline Pattern at $t=6--$ Uniform Profile 


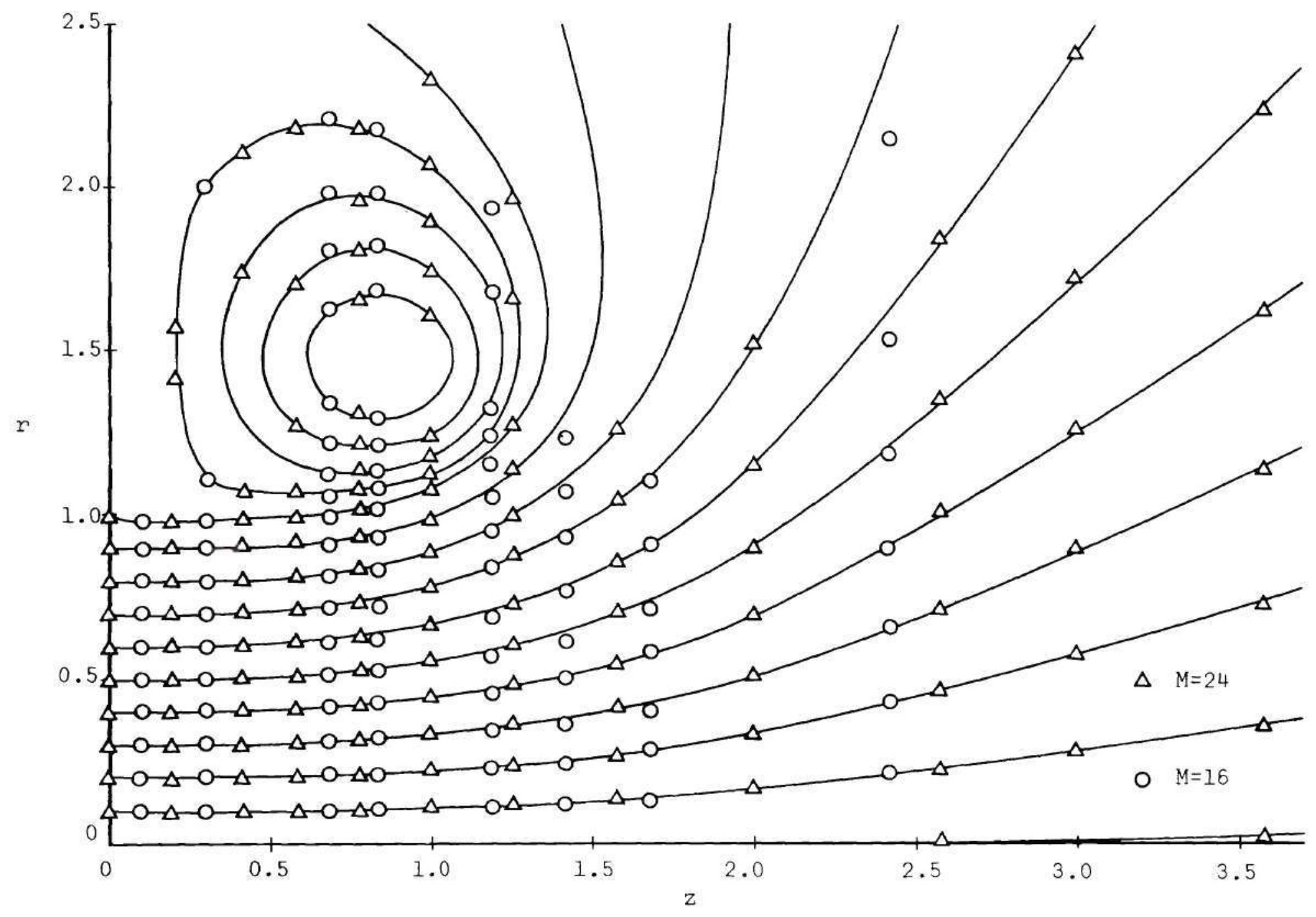

Figure H-23. Streamline Pattern at $t=10--$ Uniform Profile 


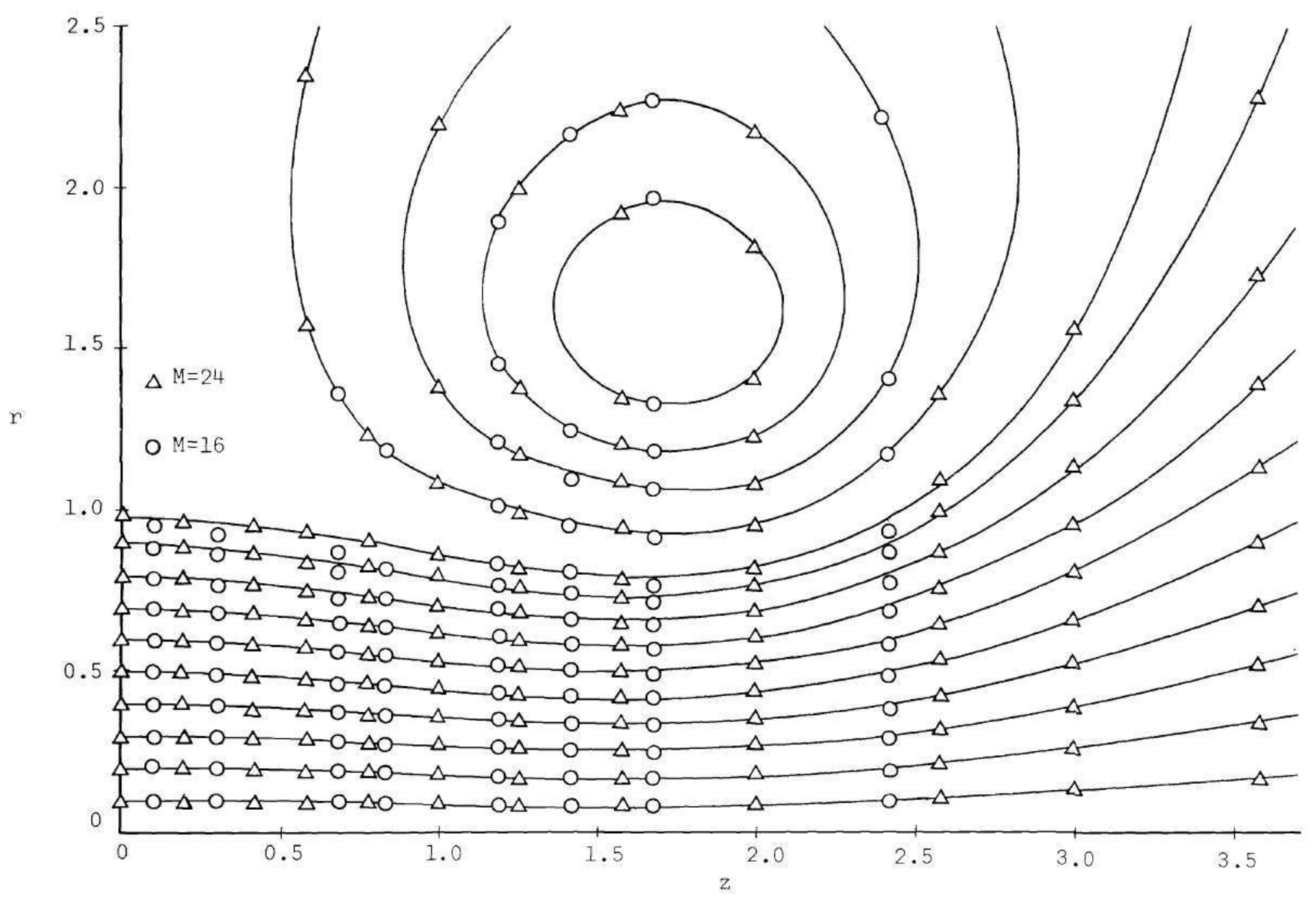

Figure H-24. Streamline Pattern at $t=14$-- Uniform Profile 


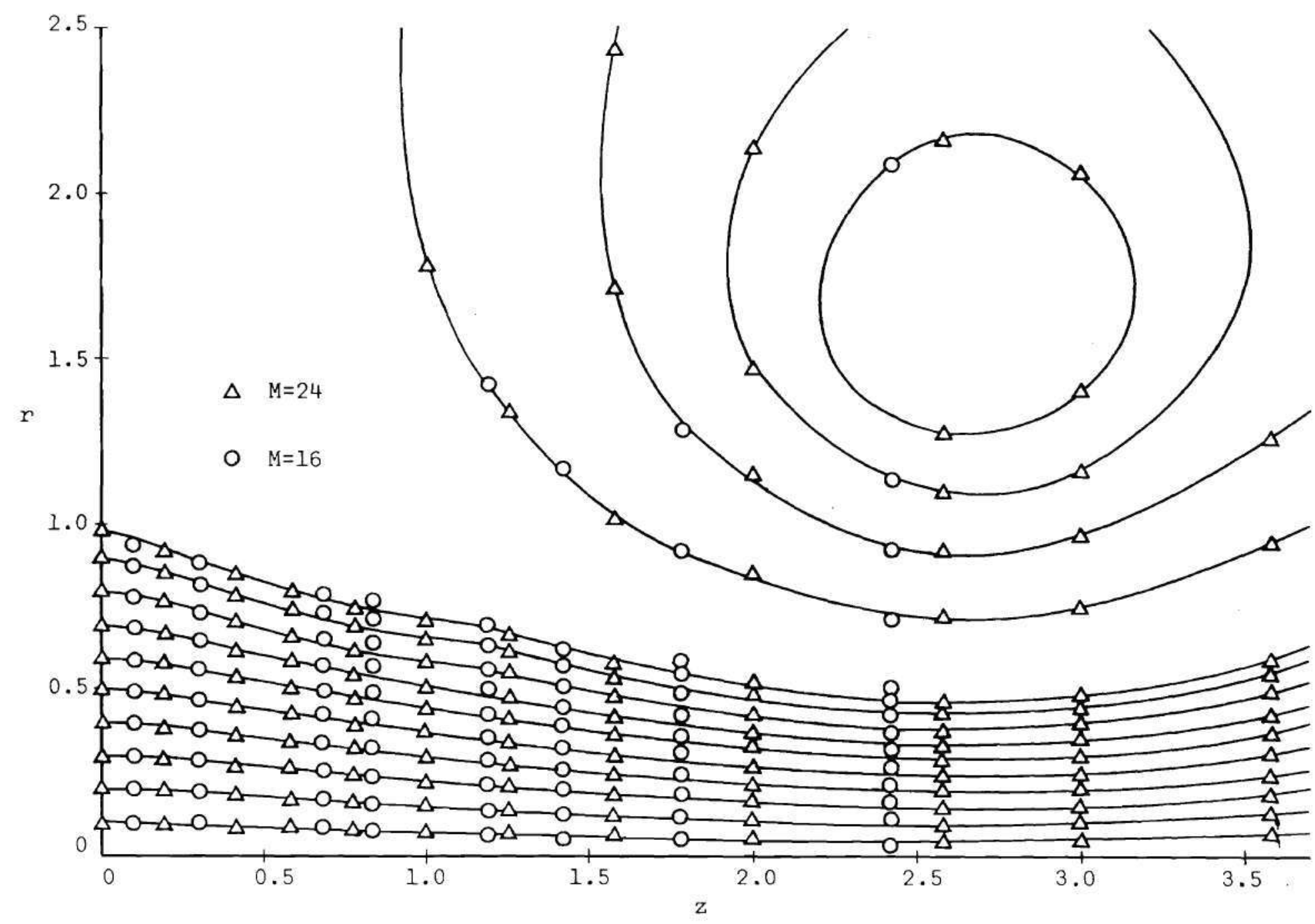

Figure H-25. Streamline Pattern at $t=18$-- Uniform Profile 


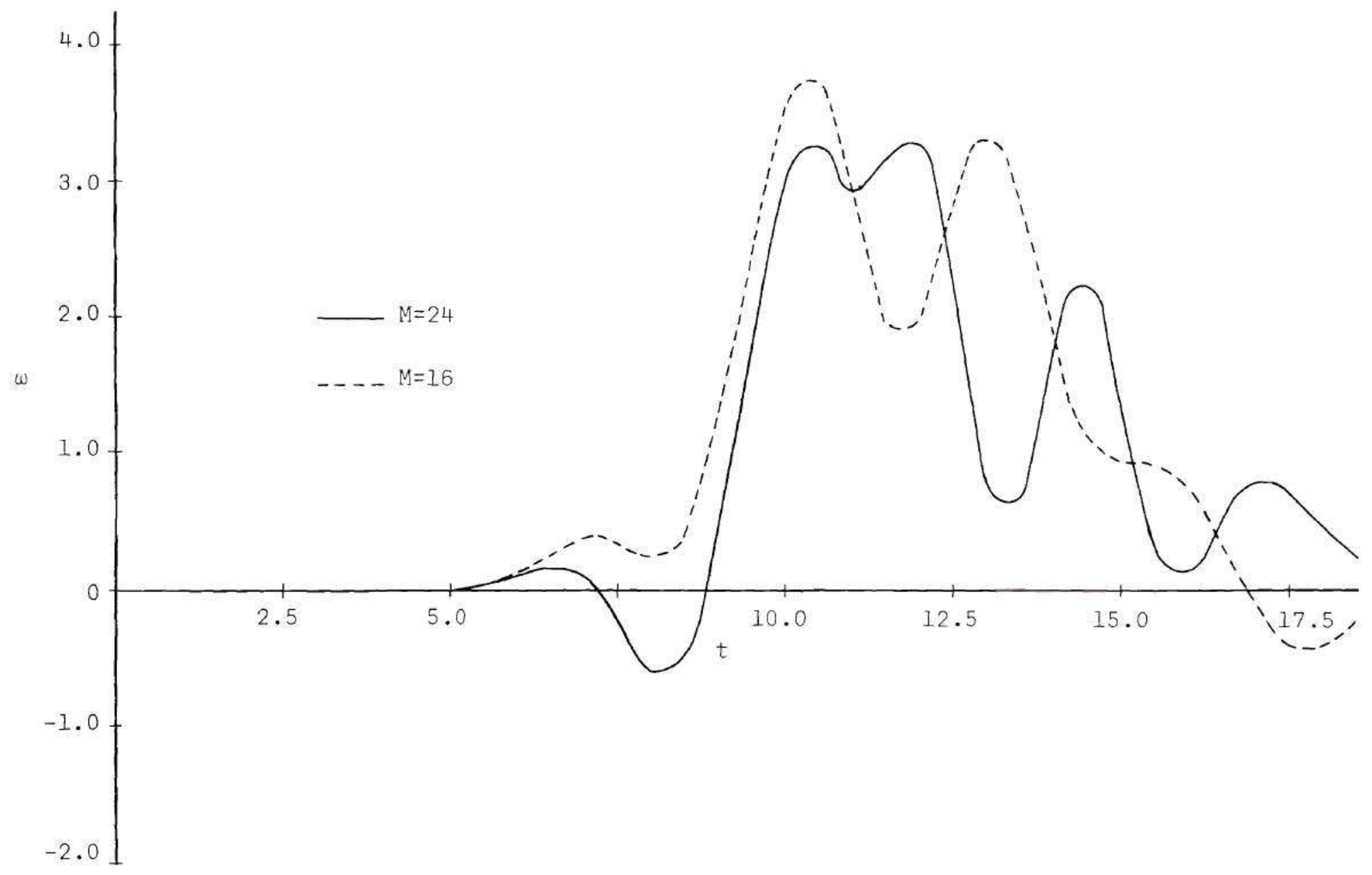

Figure H-26. Vorticity at $z=1, r=1$-- Uniform Profile 


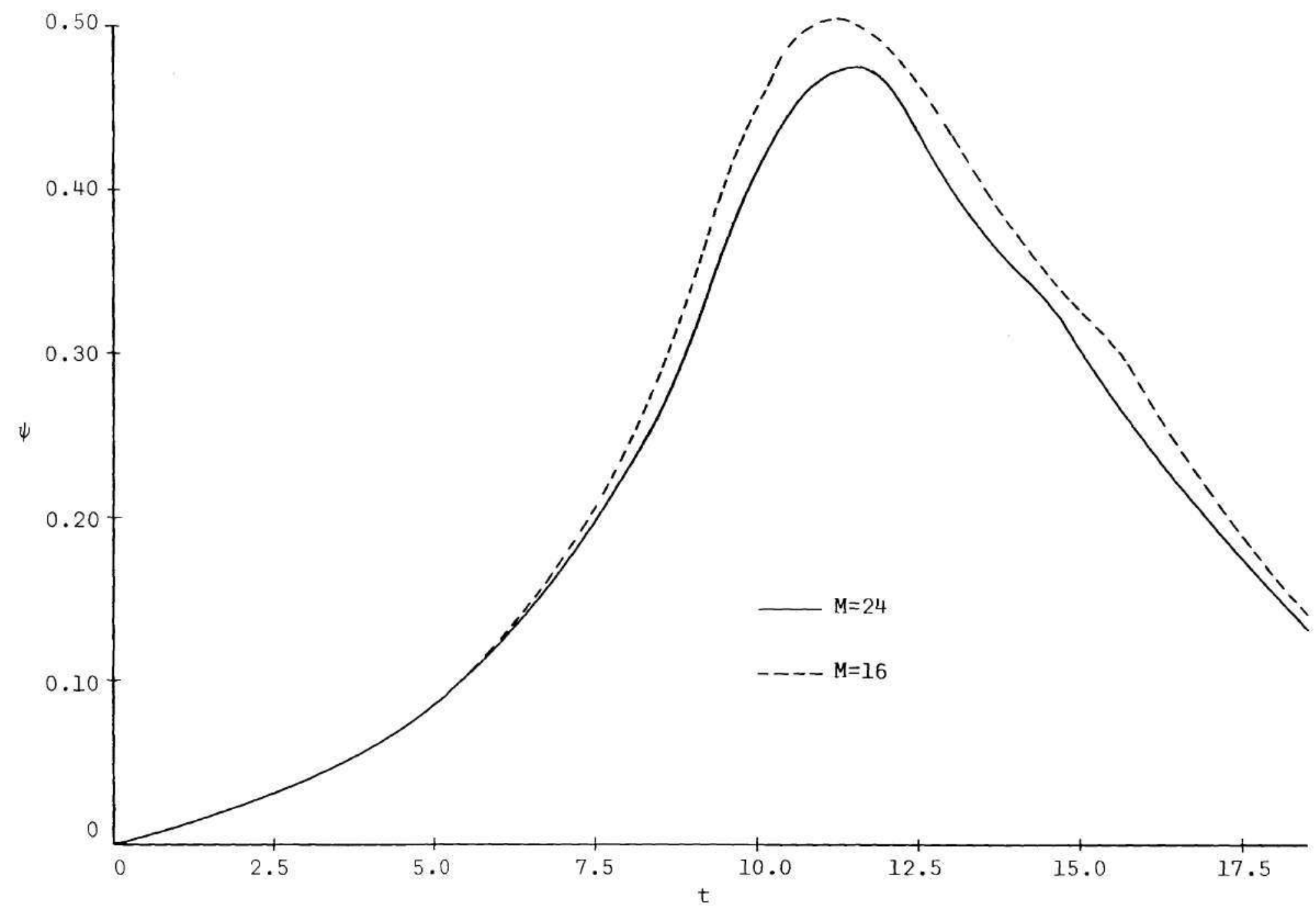

Figure H-27. Stream Function at $z=1, r=1$-- Uniform Profile 


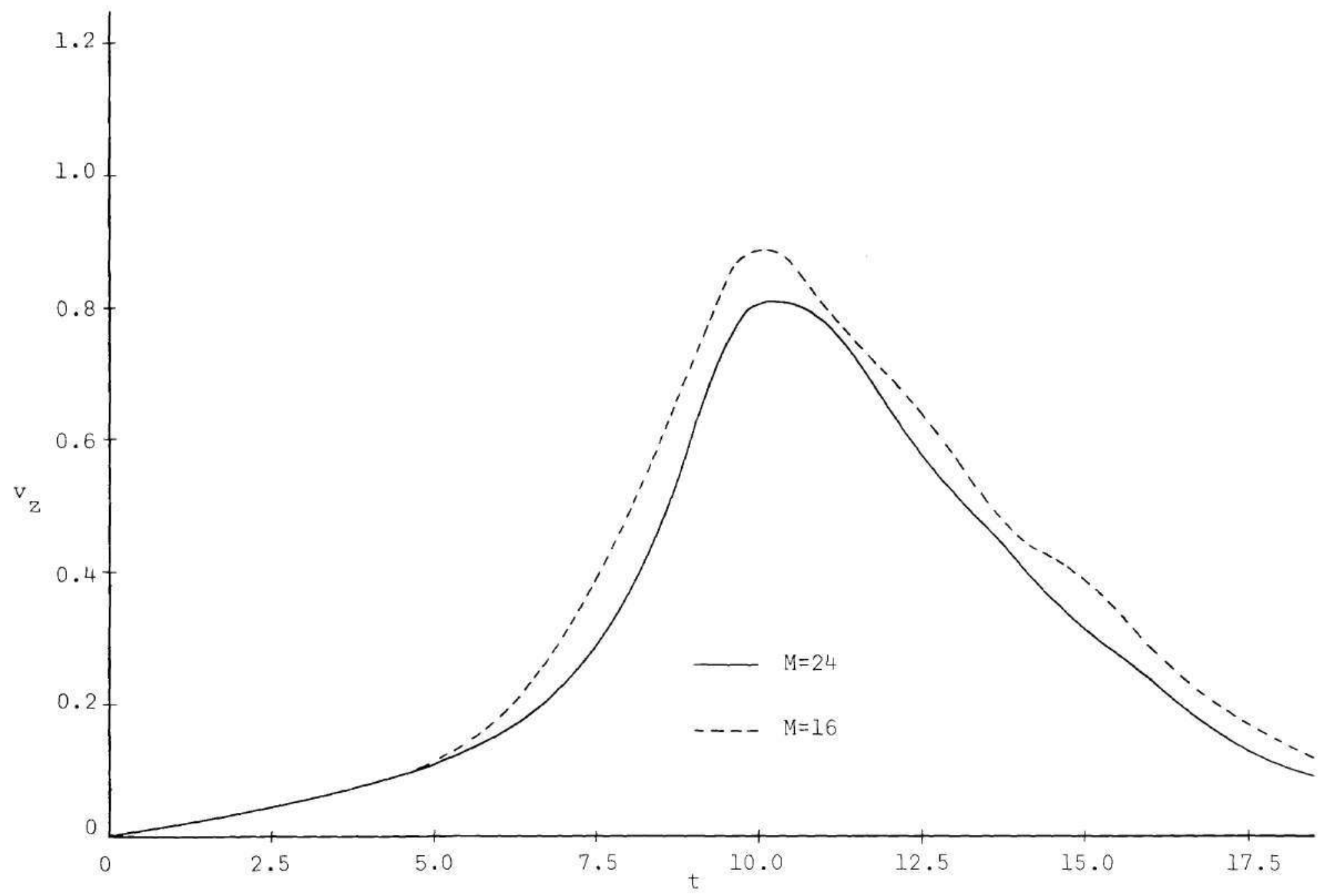

Figure H-28. Axial Velocity at $z=1, r=1$-- Uniform Profile 


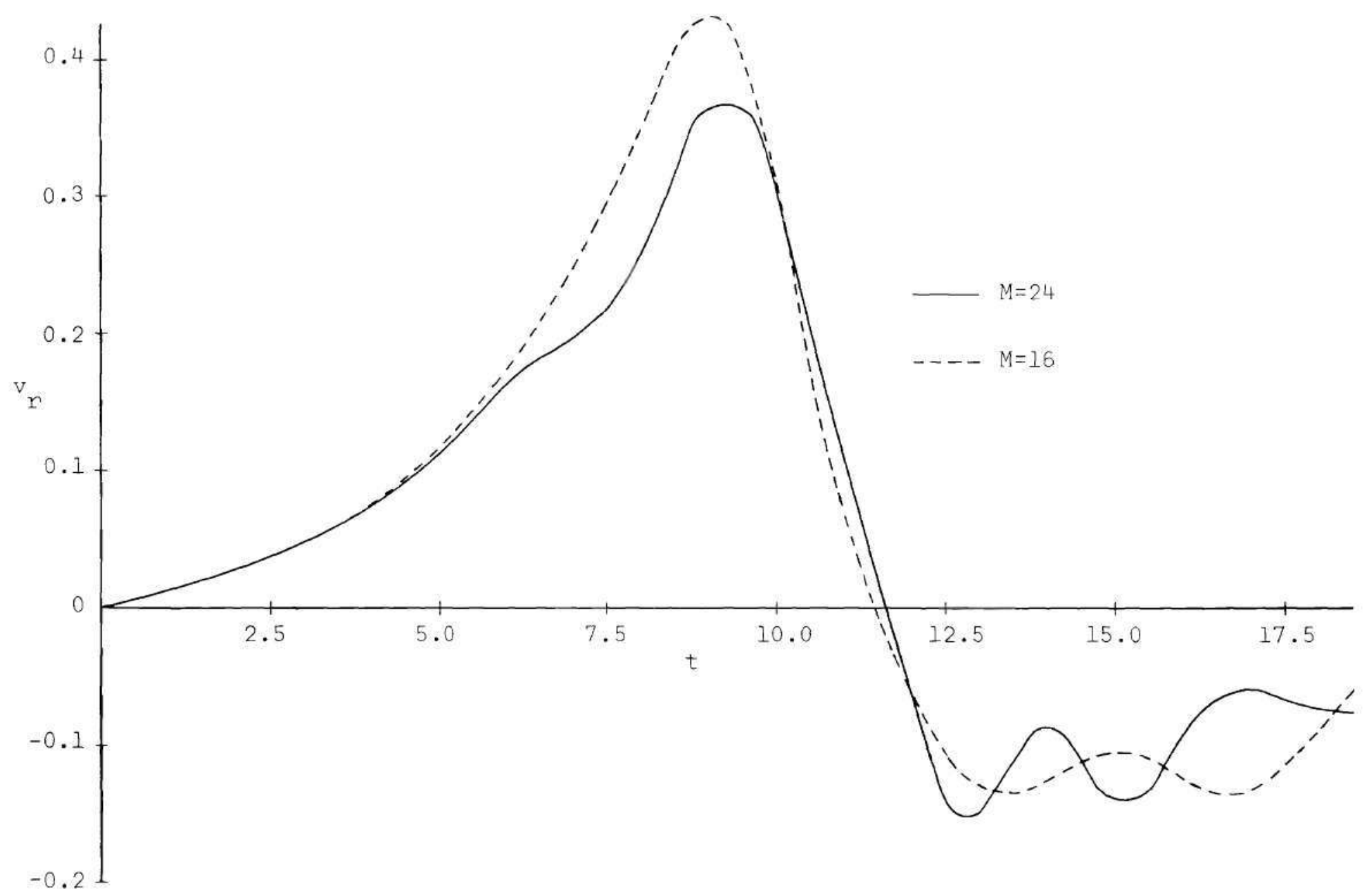

Figure H-29. Radial Velocity at $z=1, r=1--$ Uniform Profile 


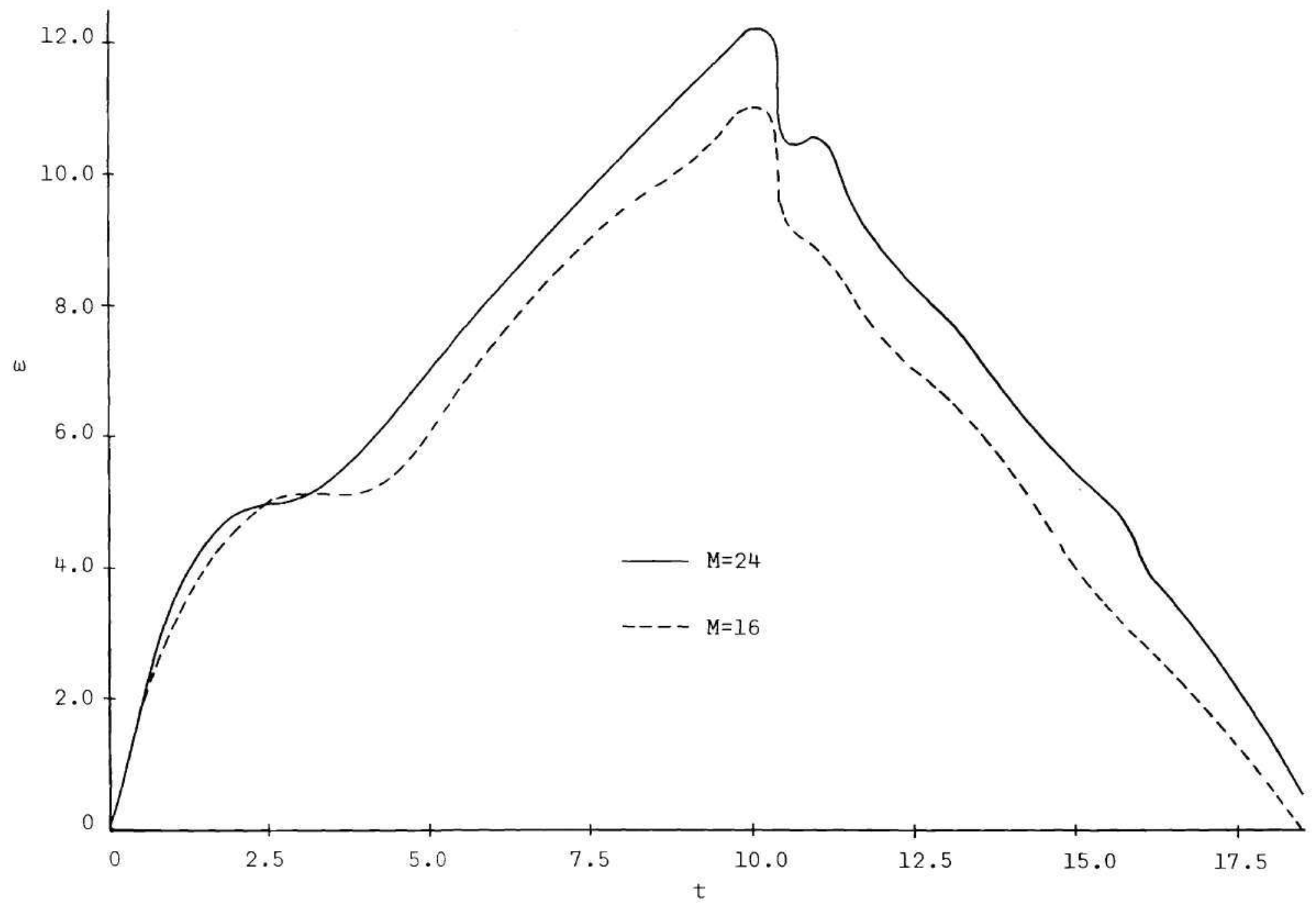

Figure H-30. Vorticity at $z=0, r=1$-- Uniform Profile 
of time in Figure $\mathrm{H}-30$.

Figures $\mathrm{H}-16$ through $\mathrm{H}-20$ illustrate good qualitative agreement for the vorticity contours plotted. In the case of the vortex ring generated by the uniform profile, fluid emerges from the exit plane possessing a negative vorticity which denotes clockwise rotation in the upper half of the meridian plane plotted in these figures. This physical phenomenon is discussed in detail in the results of chapter IV. However, due to the large gradients, these regions of negative vorticity decay and break up into smaller elements. Although these regions of negative vorticity appear in both Problem I and Problem II of the uniform profile case, the locations of corresponding areas of the same negative vorticity do not compare well all the time. Since these regions are very small, they are not shown in this series of figures because to present them adds too much detail to the figures. However, the vorticity contours which indicate the vortex ring do show good agreement.

The streamline patterns of Figures $\mathrm{H}-2 \mathrm{l}$ through $\mathrm{H}-25$ compare very well. Again, this behavion is expected. The vorticity indicates the derivatives of the stream function whereas the streamlines are simply contours of the stream function itself.

A quantitative comparison can be made from the vorticity at the point $z=1$ and $r=1$ as shown in Figure $H-26$. This point in space experiences the large fluctuations in vorticity caused by the breakup of regions of negative vorticity closer to the center line. The case for $M=24$, in which the breakup can be seen in more detail, shows the vorticity actually becoming negative as one of the elements passes. 
After the ring has passed at about $t=12$, the value of vorticity oscillates as the regions of trailing vorticity move out into the field. Figure $\mathrm{H}-27$ demonstrates the variation with time of the stream function at the same point in space. Here, the convergence of the two solutions is indicated much more clearly. The maximum deviation appears to be about 10 per cent. Figures $H-28$ and $H-29$ illustrate the variation of the axial and radial velocity components, respectively. The axial velocity shows a smoother variation for the mesh of $M=24$, whereas the radial distribution shows a more detailed oscillation as the secondary trailing vortices pass the point of interest for the mesh of $M=24$.

Finally, Figure $\mathrm{H}-30$ shows the behavior of the vorticity on the solid boundary at the edge of the orifice. The maximum vorticity occurs at the time $t=10$, and the fact that the vorticity is positive demonstrates the dominance of the term $\frac{\partial v_{z}}{\partial r}$ over the term $\frac{\partial v_{r}}{\partial z}$ at this point. Since the axial velocity profile is linear over the range $0.95 \leq r \leq 1.0$, observation of Equation (72) indicates that

$$
\frac{\partial v}{\partial r}=20
$$

at $r=1$ and $t=10$. Since the total vorticity at this point in space and time has a value of only about 12, the other component of vorticity exhibits a value which has the same order of magnitude. The agreement of the two curves of Figure $\mathrm{H}-30$ gives a further degree of confidence in the method by which the term $\frac{\partial v_{r}}{\partial z}$ is calculated numerically. 
Concluding Remarks

In this appendix, a comparison is made of the results of the numerical calculations for two different mesh sizes for both the parabolic and uniform axial velocity distributions in the exit plane. Both cases show good qualitative agreement in the region of the flow around the vortex ring. The agreement is better for the stream function than the vorticity since the vorticity denotes the behavion of various spatial derivatives of the stream function. In the case of the vortex ring generated by the uniform velocity profile, negative vorticity is generated in the exit plane, and the results of the solutions for $M=16$ and $M=24$ indicate that convergence to a high degree of accuracy has not yet been achieved in this region of the flow field. Overall, convergence in the parabolic profile case appears to be better than for the uniform profile case. This behavior is attributed to a higher truncation error in the uniform case. The gradients of the dependent variables in both space and time are greater for the uniform profile case, and this results in higher values of the derivative which is one of the components of the truncation error of each finite difference approximation. 


\section{A STEADY STATE SOLUTION}

In order to verify the model used to study the generation and development of a viscous vortex ring, it was considered desirable to compare a steady state solution of the numerical model to an existing solution. A direct comparison is prohibitively expensive though, because of the long computer time necessary to obtain a steady state solution for the center line profile of Figure I-I. However, if the numerical model preserves a known steady state solution, a degree of confidence can be established in the numerical technique. Such an existing solution of the boundary layer equations is given in Pai [36] for steady flow issuing from a round hole of infinitesimal size. For completeness, the analysis is repeated here as it is adapted to the nomenclature of this investigation and the configuration illustrated in Figure $\mathrm{I}-2$.

The steady, incompressible, axisymmetric motion is described by the boundary layer equations and the continuity equation. These are given by

$$
\begin{gathered}
v_{z}^{*} \frac{\partial v_{z}^{*}}{\partial z^{*}}+v_{r}^{*} \frac{\partial v_{z}^{*}}{\partial r^{*}}=\frac{\nu^{*}}{r^{*}} \frac{\partial}{\partial r^{*}}\left(r * \frac{\partial v_{z}^{*}}{\partial r^{*}}\right) \\
v_{z}^{*}=\frac{1}{r * \frac{\partial \psi^{*}}{\partial r}}
\end{gathered}
$$




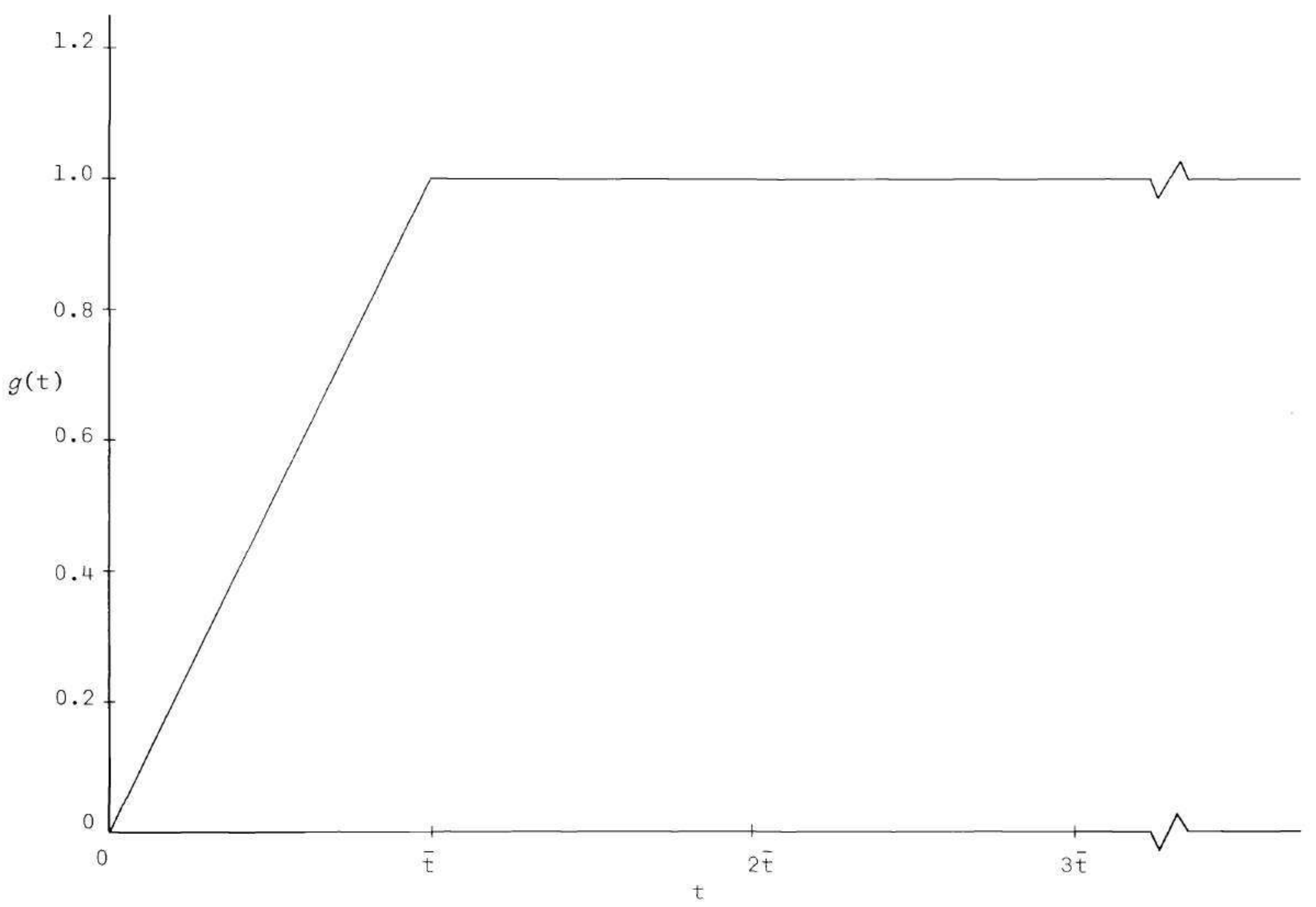

Figure I-1. Center Line Profile for Steady State Solution 


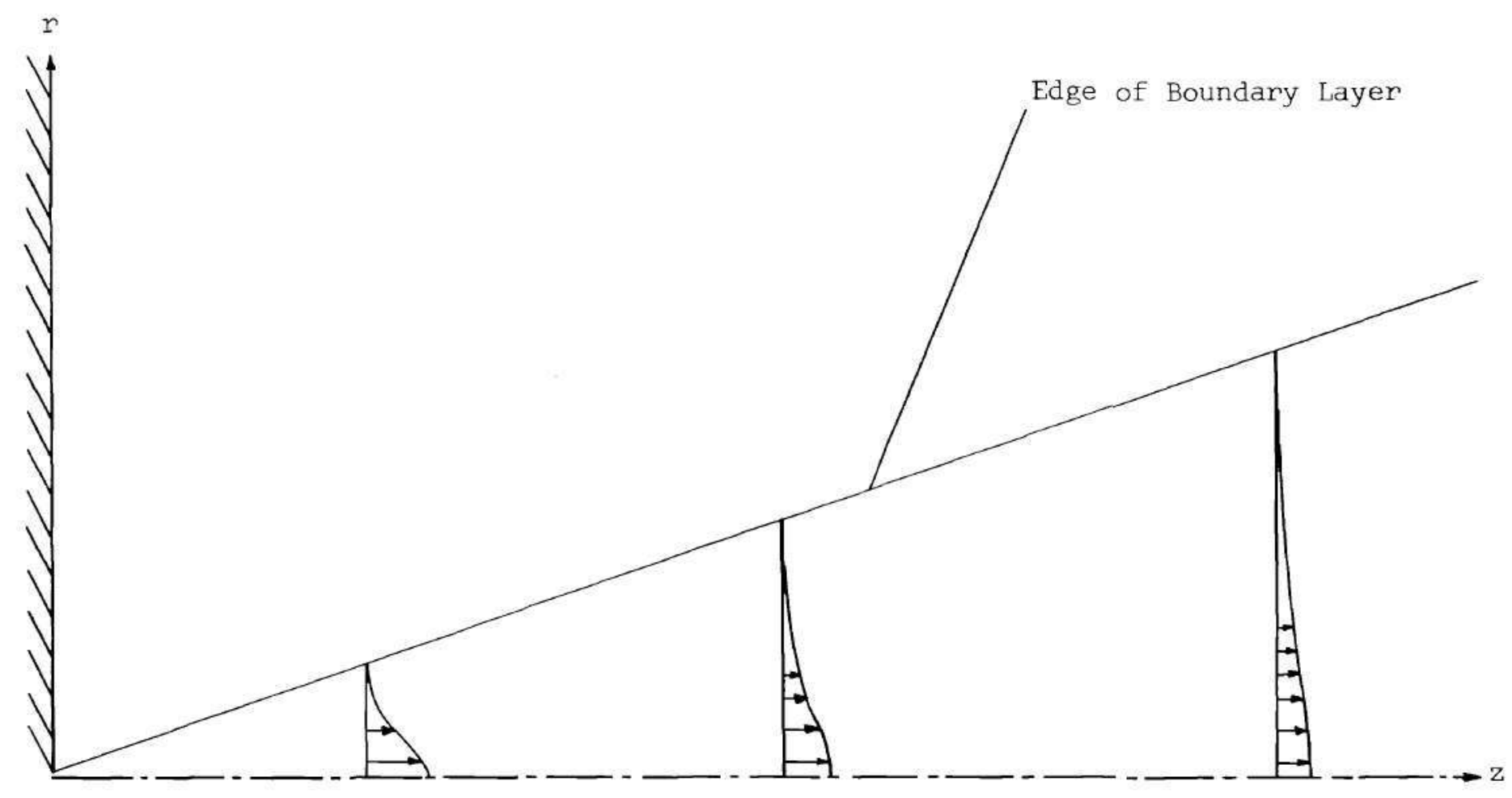

Figure I-2. Physical Configuration for Axisymmetric Flow from a Small Round Hole 


$$
v_{r}^{*}=-\frac{1}{r} \frac{\partial \psi^{*}}{\partial z^{*}}
$$

The accompanying boundary conditions are

$$
\text { at } r^{*}=\infty \quad v_{z}^{*}=0
$$

and

$$
\text { at } r^{*}=0 \quad v_{r}^{*}=\frac{\partial v^{*}}{\partial r^{*}}=0
$$

(Note that these assumptions are consistent with the numerical model of the vorticity transport equation.) The momentum flux of the jet at any section is given by

$$
M_{0}^{*}=2 \pi \rho^{*} \int_{0}^{\infty} v_{z}^{* ?} r^{*} d r *
$$

and $\mathrm{M}_{0}^{*}$ is constant. Under the assumption that there exist similar solutions, the dependent variables may be written as

$$
\begin{gathered}
\psi^{*}=v^{*} z^{*} g\left(\Phi^{*}\right) \\
v_{z}^{*}=\frac{g^{\prime}\left(\Phi^{*}\right)}{\Phi^{*} z^{*}} \\
v_{r}^{*}=\frac{\sqrt{v^{*}}}{z^{*}}\left[g^{\prime}\left(\Phi^{*}\right)-\frac{g\left(\Phi^{*}\right)}{\Phi^{*}}\right]
\end{gathered}
$$

where

$$
\Phi^{*}=\frac{1}{\sqrt{v *}} \frac{r^{*}}{z^{*}}
$$


and the prime denotes differentiation with respect to the independent variable. Substituting these equations into Equation ( $I-1$ ) gives the differential equation for the unknown function $g$, i.e.

$$
-\frac{\mathrm{d}}{\mathrm{d} \Phi^{*}}\left(\frac{\mathrm{gg} \Phi^{\prime}}{\Phi^{*}}\right)=\frac{\mathrm{d}}{\mathrm{d} \Phi^{*}}\left(\mathrm{~g}^{\prime \prime}-\frac{\mathrm{g}^{\prime}}{\Phi^{*}}\right)
$$

The transformed boundary conditions become

$$
\begin{array}{ll}
\text { at } \Phi^{*}=\infty & g^{\prime}\left(\Phi^{*}\right)=0 \\
\text { at } \Phi^{*}=0 & \mathrm{~g}\left(\Phi^{*}\right)=\mathrm{g}^{\prime}\left(\Phi^{*}\right)=0
\end{array}
$$

Since the arguments of both derivatives with respect to $\Phi^{*}$ in Equation (I-7) must vanish at the origin, Equation (I-7) may be immediately integrated to give

$$
\Phi^{*} g^{\prime \prime}-g^{\prime}+g g^{\prime}=0
$$

which has the solution

$$
g(\tau)=\frac{\tau^{2}}{1+\left(\tau^{2} / 4\right)}
$$

where

$$
\tau=C \Phi^{*}
$$


and $c$ is an arbitrary constant which may be determined from

$$
M_{0}^{*}=\left(\frac{16}{3}\right) \pi \mu^{*} c^{2}
$$

The velocities may now be written as

$$
v_{z}^{*}=\frac{3 M_{0}^{*}}{8 \pi \mu^{*}} \frac{1}{z^{*}} \frac{1}{\left[1+\left(\tau^{2} / 4\right)\right]^{2}}
$$

and

$$
v_{r}^{*}=\frac{1}{4}\left(\frac{3 M_{0}^{*}}{\pi \rho}\right)^{\frac{1}{2}} \frac{1}{z} \frac{\tau\left[1-\left(\tau^{2} / 4\right)\right]}{\left[1+\left(\tau^{2} / 4\right)\right]^{2}}
$$

where

$$
\tau=\frac{1}{4 \nu^{*}}\left(\frac{3 M_{0}^{*}}{\pi \rho^{*}}\right)^{\frac{1}{2}} \frac{r^{*}}{z^{*}}
$$

It is also seen from the solution that the stream function distribution is given by

$$
\psi^{*}=\nu^{*} z^{*} \frac{\tau^{2}}{1+\tau^{2} / 4}
$$

This may be verified by calculating the velocities and comparing them to the ones given above. Pai also notes that the approximate solution is good as long as $\mathrm{M}_{0}^{*} / \mathrm{p}^{*} v^{* 2}$ is large. Rewriting these results in terms of the dimensionless variables used throughout this study gives 


$$
\begin{gathered}
v_{z}=\frac{3}{8} M_{0} \operatorname{Rey} \frac{1}{z} \frac{1}{\left[1+\left(\tau^{2} / 4\right)\right]^{2}} \\
v_{r}=\frac{1}{4}\left(3 M_{0}\right)^{\frac{1}{2}} \frac{1}{z} \frac{\tau\left[1-\left(\tau^{2} / 4\right)\right]}{\left[1+\left(\tau^{2} / 4\right)\right]^{2}} \\
\psi=\frac{z}{\operatorname{Rey}} \frac{\tau^{2}}{\left[1+\left(\tau^{2} / 4\right)\right]}
\end{gathered}
$$

where

$$
\begin{gathered}
\tau=\frac{R e y}{4}\left(3 \mathrm{M}_{0}\right)^{\frac{1}{2}}\left(\frac{r}{\mathrm{z}}\right) \\
\mathrm{M}_{0}=\frac{\mathrm{M}_{0}^{*}}{\pi \mathrm{R}^{2} \rho^{*} \mathrm{U}^{2}}=2 \int_{0}^{\infty} \mathrm{v}_{\mathrm{z}}^{2} \mathrm{rdr}
\end{gathered}
$$

and $R$ and $U$ are respectively the dimensional radius of the exit plane and the dimensional maximum center line velocity.

These results must now be adapted to the control volume of Figure 2. To do this, assume the existence of a fictitious axial distance $\bar{z}$ such that

$$
\begin{gathered}
\mathrm{v}_{\mathrm{z}}(\bar{z}, 0)=1 \\
\mathrm{v}_{\mathrm{z}}(\bar{z}, I)=K \cong 0
\end{gathered}
$$

The flow field resulting from these relations is shown in Figure I-3. Now, investigate the effect of these conditions. Consider the first, Equation (I-15). Erom Equation $(I-13), \tau=0$ at $r=0$, and when Equation 


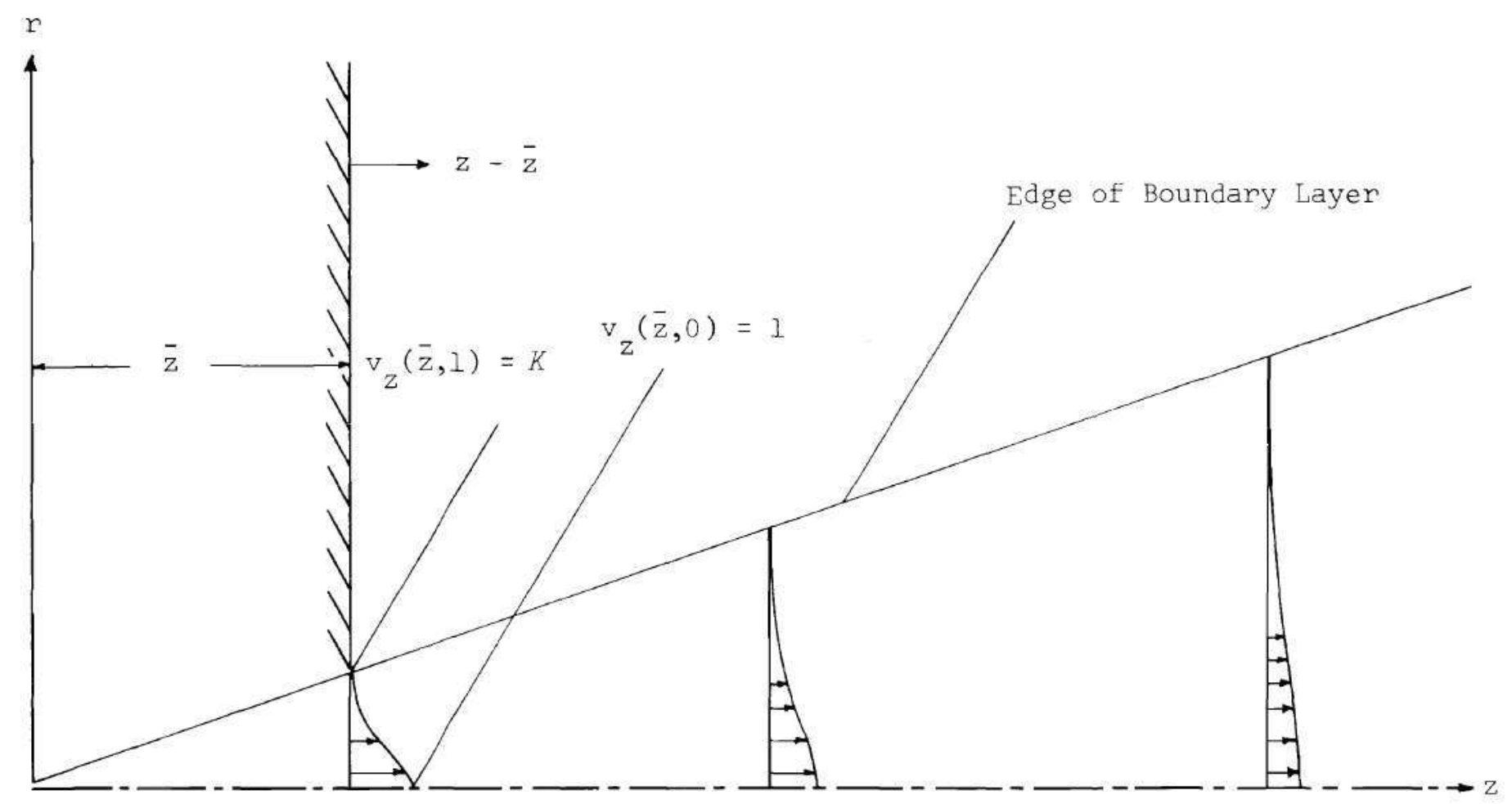

Figure I-3. Physical Configuration for Axisymmetric Flow from a Finite Round Hole 
(I-10) is simplified by this fact and combined with Equation (I-15), there results the expression

$$
\mathrm{v}_{\mathrm{z}}(\bar{z}, 0)=1=\frac{3}{8} M_{0} \operatorname{Rey} \frac{1}{\bar{z}}
$$

from which it may be seen that

$$
\bar{z}=\frac{3}{8} M_{0} R e y
$$

Consider next the effect of Equation (I-16). Combining it with Equation (I-10) and employing Equation ( $I-17$ ) yields

$$
\mathrm{v}_{z}(\bar{z}, 1)=K=\frac{1}{\left[1+\left(\tau^{2} / 4\right)\right]^{2}}
$$

where at $z=\bar{z}$ and $r=1$ the expression for $\tau$ reduces to

$$
T(\bar{z}, 1)=\frac{R e y}{4}\left(3 M_{0}\right)^{\frac{1}{2}} \frac{1}{\bar{z}}
$$

Simplifying this with the aid of Equation (I-17) allows the derivation of

$$
\tau(\bar{z}, 1)=\left(\frac{R e y}{2 \bar{z}}\right)^{\frac{1}{2}}
$$

which when substituted into Equation ( I-18) produces the equation 


$$
K=\frac{1}{\left[1+\frac{1}{8} \frac{R e y}{z}\right]^{2}}
$$

After selecting the positive root for physical reasons, the distance $\bar{z}$ may be computed as

$$
\bar{z}=\frac{1 / 8 R e y}{(1 / K)^{1 / 2}-1}
$$

Hence, the fictitious axial distance $\bar{z}$ is fully specified by the Reynolds number and the "slip" velocity $K$ at the edge of the exit plane. (The Prandtl no-slip condition is not satisfied exactly by this analysis, but if $K$ is chosen small enough, the effect of the slip should be small.)

Once a value of the parameter $K$ has been adopted, the axial distance $\bar{z}$ may be computed by Equation ( I-19) and the momentum flux of the fluid at any cross-section may be determined from Equation (I-17). If the axial distance $z$ is replaced by the distance $(z-\bar{z})$ in Equations (I-10) through (I-12), there result the approximate velocity and stream function distributions for an axisymmetric free jet issuing from a round hole of radius $R$. In fact, from this point on, the spatial co-ordinate $z$ occurring in Equations ( I-10) through ( I-13) should be thought of as the axial distance between a point in the flow field and the solid boundary or the exit plane.

$$
\text { Since the dependent variables of the numerical model are the }
$$
stream function and the vorticity, the vorticity must be determined. The definition of vorticity used here is once again 


$$
\omega \triangleq \frac{\partial v_{r}}{\partial z}-\frac{\partial v_{z}}{\partial r}
$$

This may be evaluated either by substituting the velocities into this equation and differentiating or by replacing the velocities by equivalent expressions in terms of the stream function and differentiating the result. In either case the result is the same, namely

$$
\omega=\frac{3}{8} M_{0} \operatorname{Rey} \frac{1}{\left[1+\left(\tau^{2} / 4\right)\right]^{3}} \frac{1}{r z}\left\{\left(\frac{r}{z}\right)^{2}\left[3\left(\tau^{2} / 4\right)-1\right]+\tau^{2}\right\}
$$

Remember that here again the spatial co-ordinate $z$ should be recognized as the axial distance between a point in the field and the solid boundary on the exit plane.

Pai states that the approximate solution is good as long as the parameter $M_{0}^{*} / \rho^{*} v^{* 2}$ is large. When this is written in the dimensionless variables, it becomes

$$
\frac{1 / 3 \pi R e y^{2}}{(1 / K)^{1 / 2}-1} \text { must be large. }
$$

For the constant $K$ fairly small and the Reynolds number of this study, no difficulties arise.

In order to demonstrate the validity of the numerical model, the parameters of the flow field are chosen to correspond to those of the rest of this study. They are 


$$
\mathrm{U}=2 \mathrm{feet} / \mathrm{sec} \quad \mathrm{R}=0.04 \text { feet } \quad \text { Rey }=533
$$

The parameter $K$ is specified to be

$$
K=0.01
$$

The vorticity and stream function distributions were calculated from Equations (I-20) and (I-12), and the results are shown in Figures I-4 and $\mathrm{I}-5$.

The numerical model was set up for $M=24$ (529 interior mesh points) in the transformed $\xi, n$ plane, and the stream function distribution corresponding to the vorticity distribution of Equation ( I-20) was computed numerically. This is shown in Figure I-6. The numerical method was then implemented for 12 time steps with $\delta t=0.5$, and the resulting vorticity and stream function distributions are shown in Eigures I-7 and I-8. The vorticity distribution given by Equation (I-20) (shown in Figure I-4) compares so well with the vorticity distribution obtained numerically at the end of 12 time steps (Figure I-7) that no difference can be seen between the plotted results. In addition, the final stream function distribution (Figure I-8) cannot be distinguished from the initial stream function distribution (Figure I-6) which was calculated numerically to correspond with the vorticity distribution of Equation $(I-20)$. This procedure demonstrates that the numerical model preserves an appropriate solution of the boundary layer equations. In contrast, comparison of Figures I-5 and I-6 reveals a differ- 


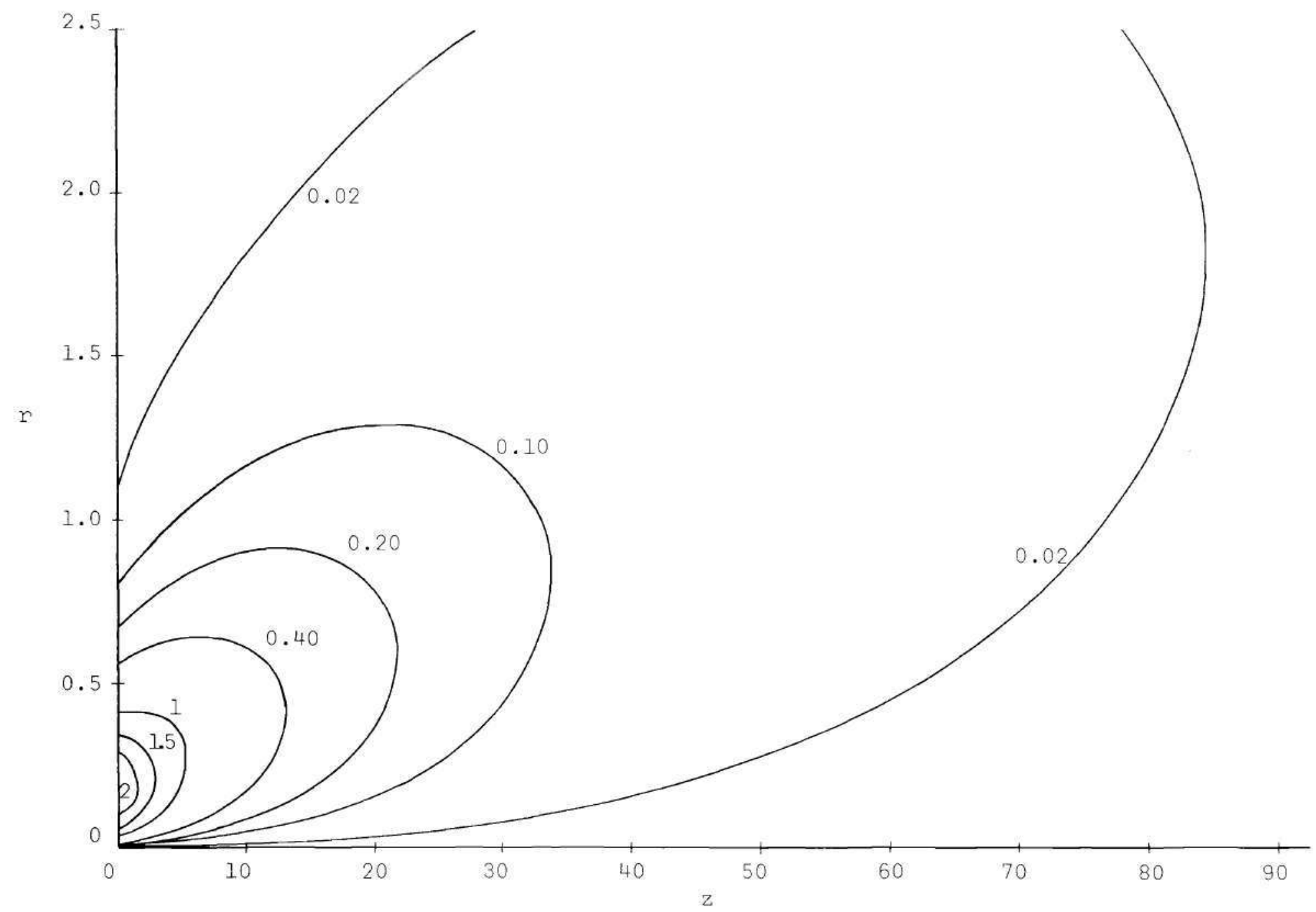

Figure I-4. Vorticity Distribution of the Boundary Layer Solution 


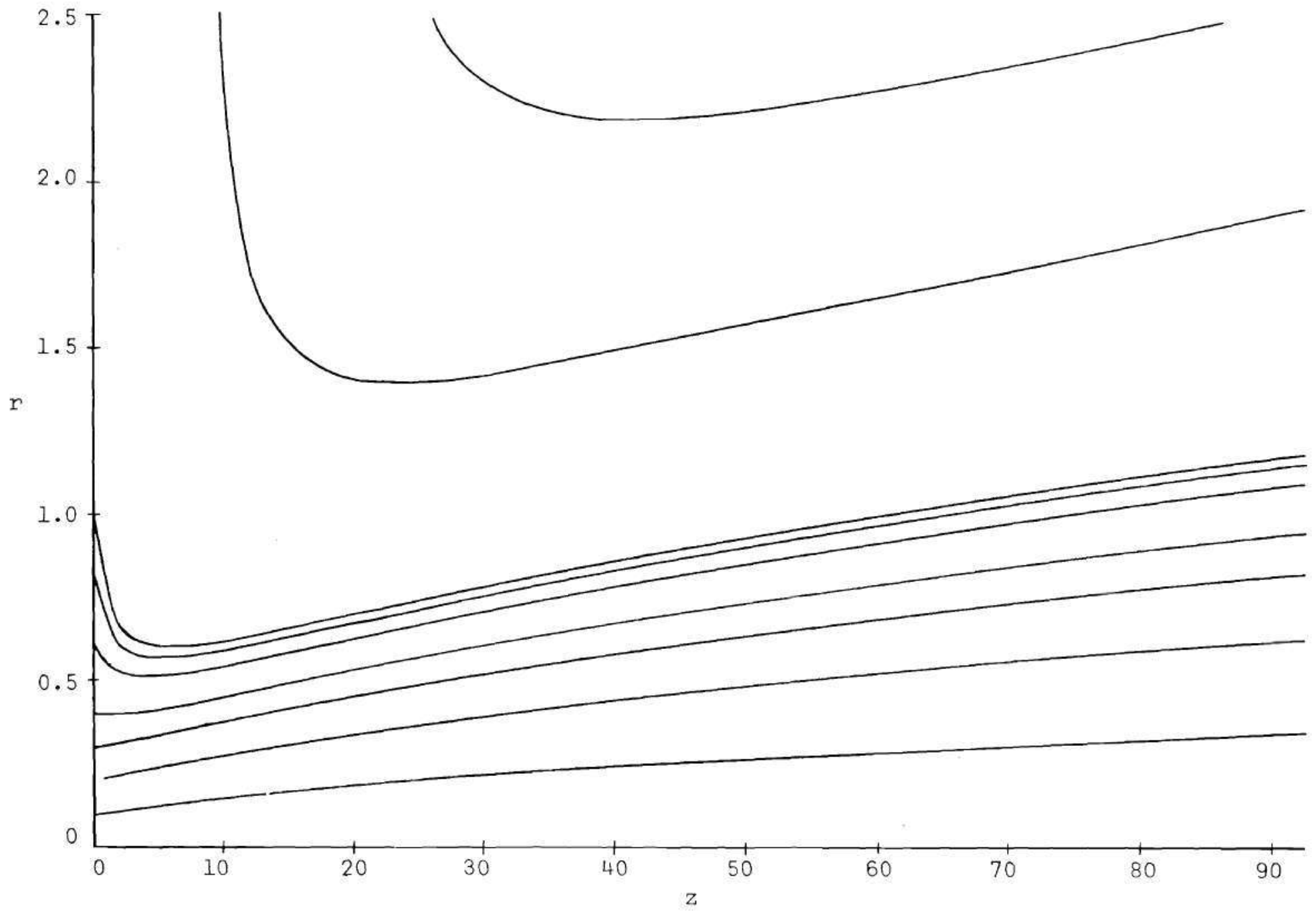

Figure I-5. Streamline Pattern of the Boundary Layer Solution 


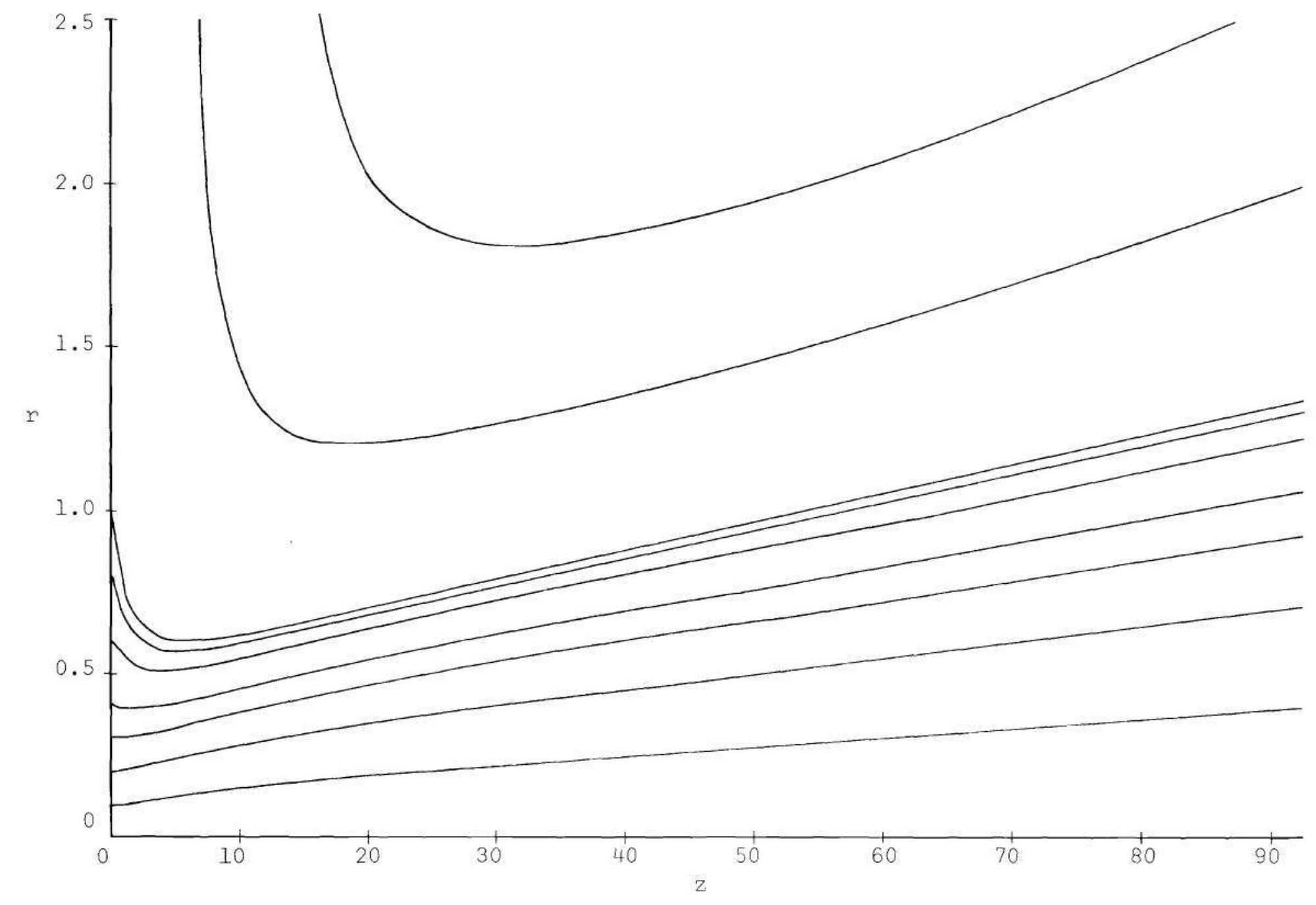

Figure I-6. Streamline Pattern of the Numerical Solution at $t=0, M=24$ 


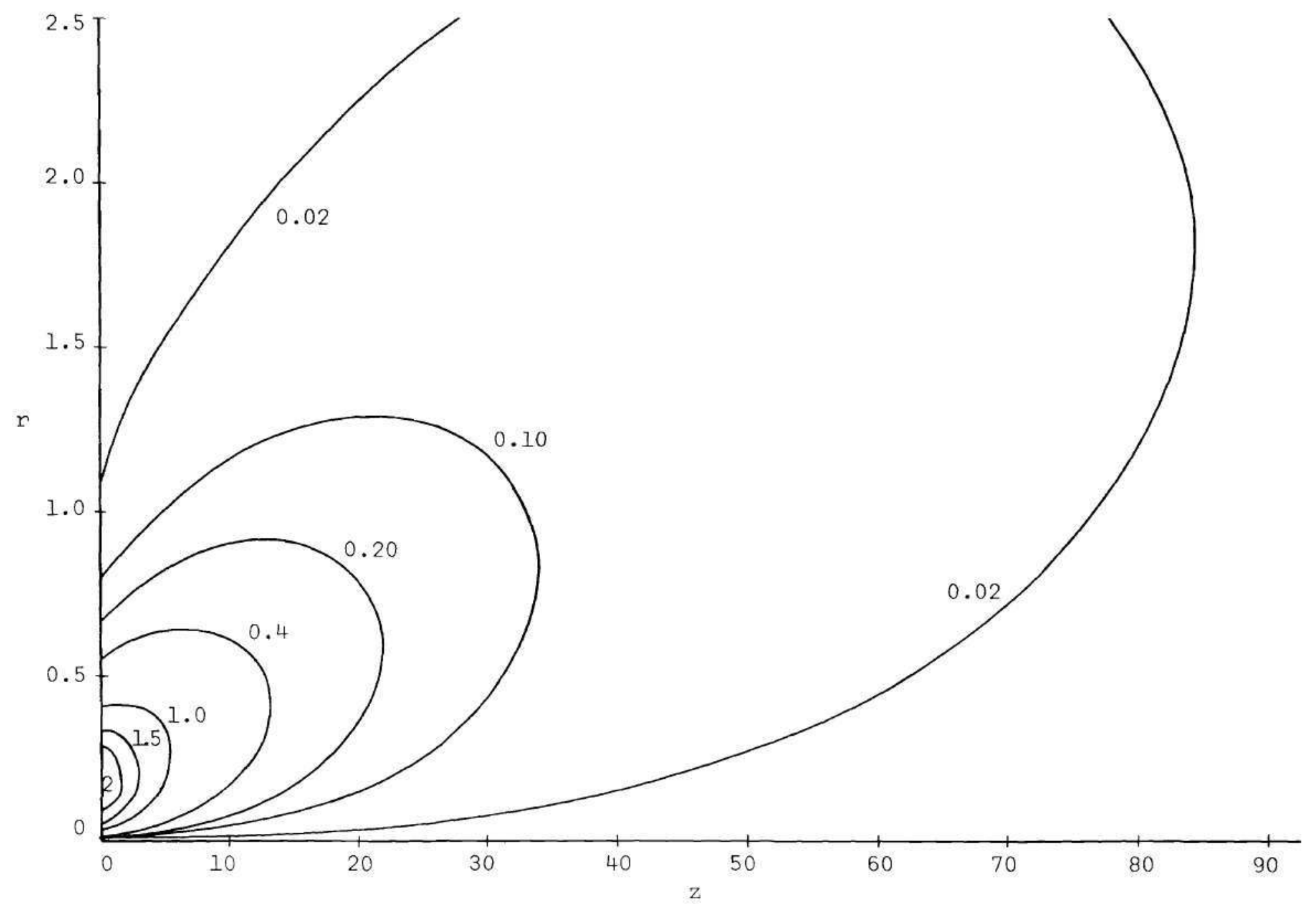

Figure I-7. Vorticity Distribution of the Numerical Solution at $t=6, \quad M=24$ 


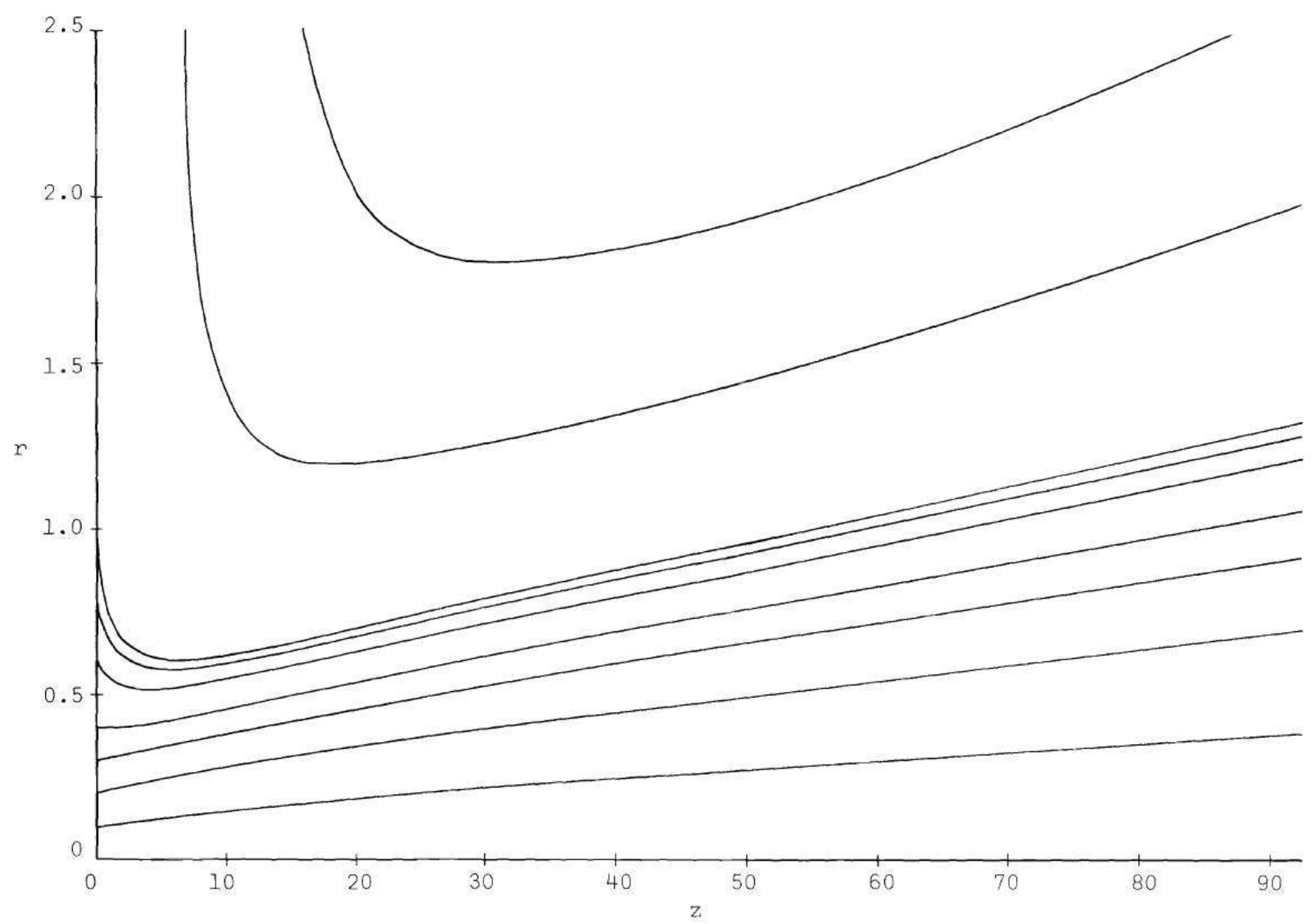

Figure I-8. Streamline Pattern of the Numerical Solution at $t=6, M=24$ 
ence in stream function distributions. Remember that the former is the distribution calculated from Equation ( $I-12$ ) whereas the latter is the numerically computed distribution which corresponds to the vorticity given by Equation (I-20). This difference can be attributed to mesh spacing alone. The parameter $M$ was increased to 50 (2401 interior mesh points) and a stream function distribution corresponding to the vorticity of Equation ( $I-20$ ) was calculated. This result is given in Figure I-9, and a comparison of this figure and Figure I-5 (the distribution of Equation ( $I-12)$ ) shows no difference between the two. This study of the axisymmetric free jet not only gives confidence to the numerical method but also substantiates another numerical approximation, the computation of the boundary value of vorticity. No difficulties occurred in this respect.

In conclusion, the steady state numerical solution compares well to the corresponding solution of the boundary layer equations. For a problem like the one studied here for which the solution to both models should be valid, the fact that the numerical method preserved the solution of the boundary layer equations indicates that the numerical model is a good one. 


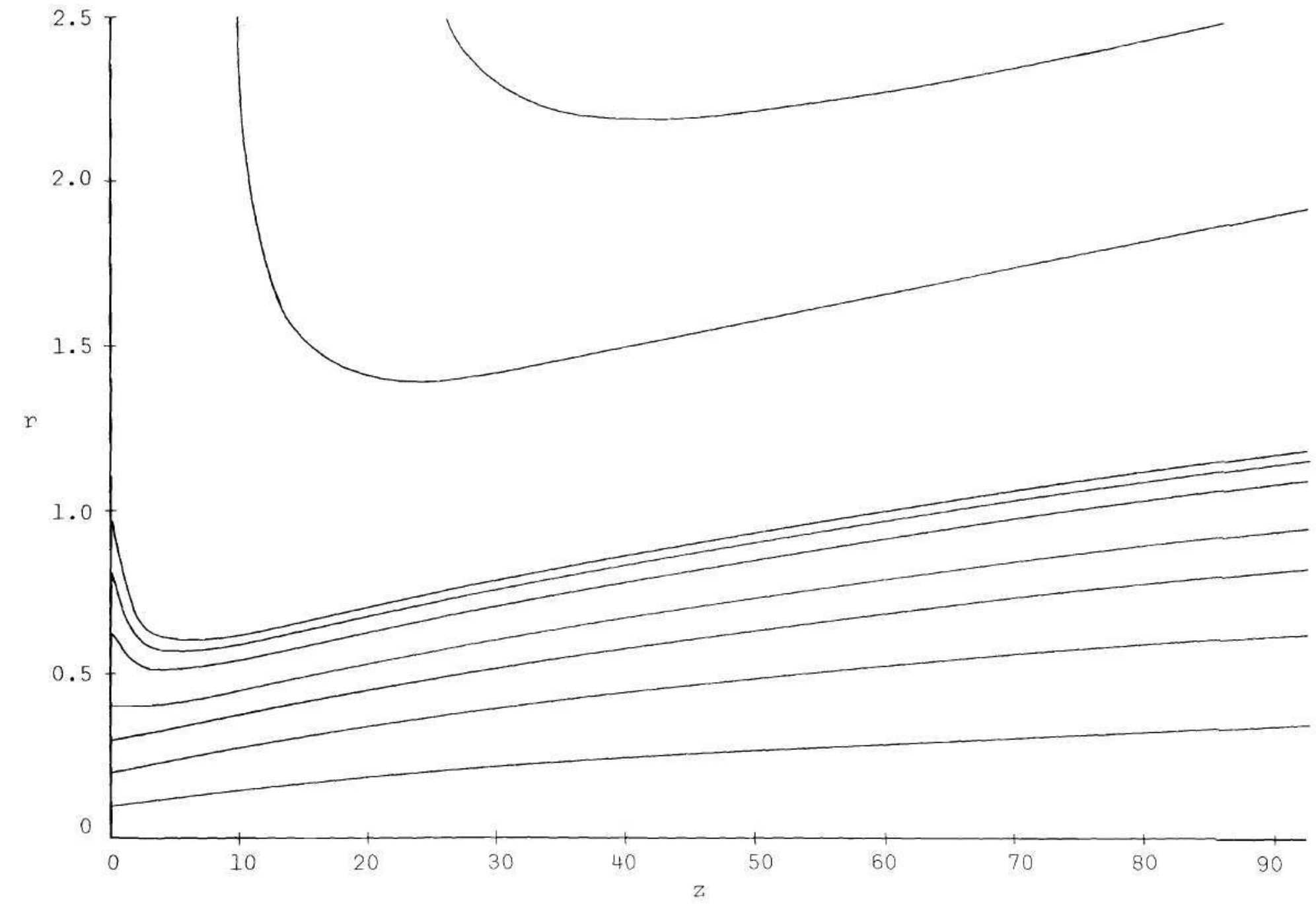

Eigure I-9. Streamline Pattern of the Numerical Solution at $t=0, M=50$ 
APPENDIX J

DEVELOPMENT OF LAMB'S VORTEX MODEL

In this appendix, the theory is developed for the vortex ring model which is usually attributed to Lamb [7]. The importance of this model is attested by its frequent use in the study of flow fields composed of circular or helical vortex patterns. It is one model which allows the prediction of the self-induced velocity of a ring.

The ring is assumed to be generated by an instantaneous impulse, and the momentum of the ring remains constant for all time. No mechanism such as viscosity exists to allow the decay of the vorticity or circulation of the ring. The derivation of the motion of the ring follows closely the work of Lamb although in this appendix the properties of the ring and the flow field are dimensionless parameters in keeping with the other analyses of this study. The development is based mainly upon energy concepts.

Consider half of the axisymmetric vortex ring, illustrated in Figure $J-1$. The ring may be thought of as being composed of a large number of closed, circular vortex filaments a few of which are shown as dots in the cross section. The most important assumptions of this analysis concern the composition and geometry of this ring. Although some of these are not needed immediately, they are presented here so that their significance can be fully established. First, it is assumed that all the vortex filaments lie within a region of circular 


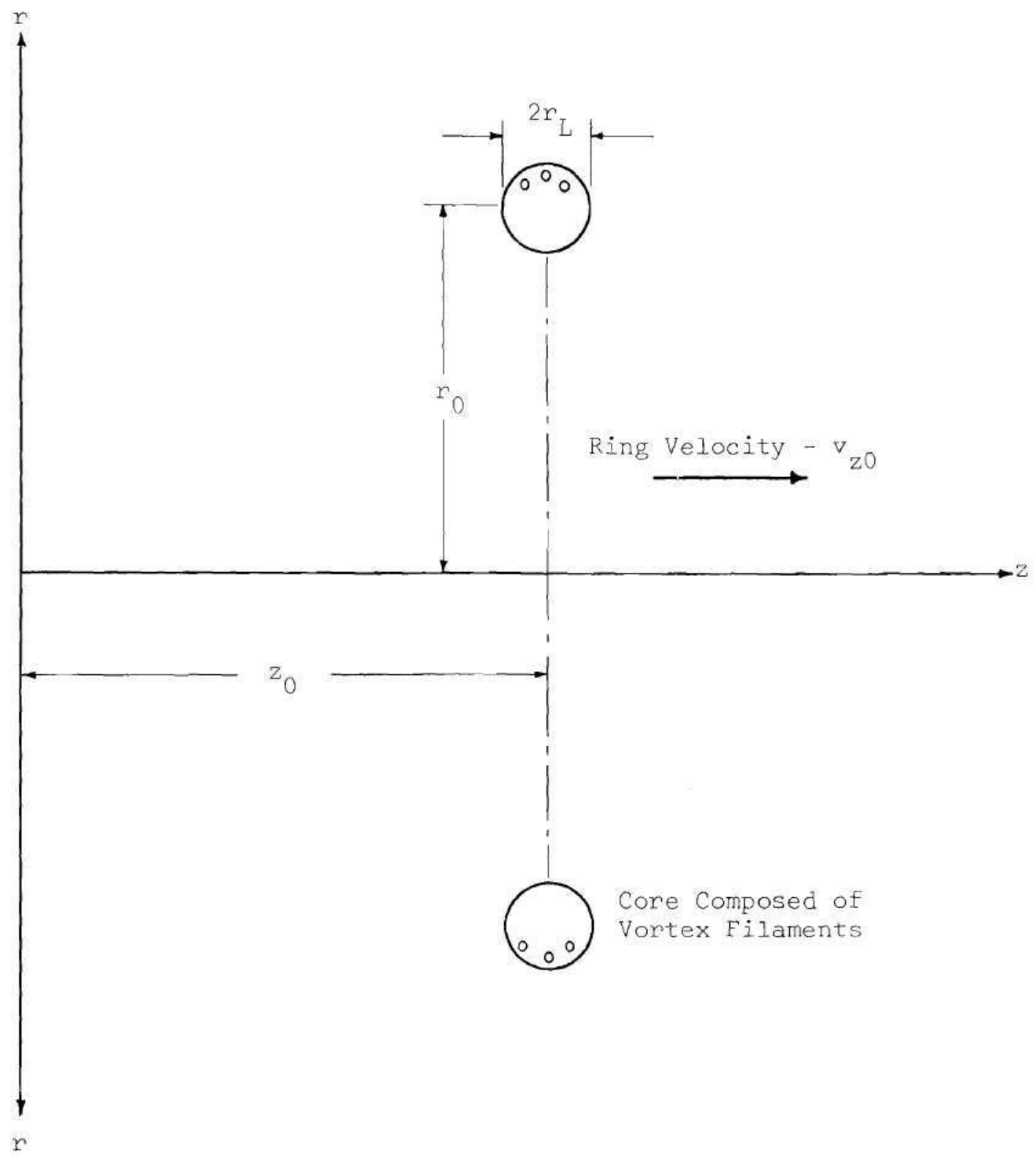

Figure J-1. Orientation of Vortex Core 
cross section. This means that the flow is rotational within the ring and irrotational outside it. The fact that the cross section is circular allows introduction of polan co-ordinates within the ring and the subsequent simplification of integration over the ring. Next, the vortex filaments are assumed to possess equal strength which does not vary with time. This simplifies the problems of integration. Since the vorticity is now constant throughout the ring, the ring rotates as a solid body, and the rotational velocity of the ring is zero at its center. (One of the characteristics of a vortex filament is the infinite velocity at the filament.) This property also satisfies the behavior of a real vortex ring which should exhibit a vanishing rotational velocity at its center. Finally, the radius of the core of the ring is assumed small with respect to the radius of the ring. The importance of this assumption is primarily in permitting the simplification of two elliptic integrals which occur later. These assumptions will be mentioned throughout the analysis whenever they are applied.

The kinetic energy of the vortex can be expressed by the equation

$$
\mathrm{T}=\frac{1}{2} \iiint\left(u^{2}+v^{2}+w^{2}\right) \mathrm{d} x \mathrm{~d} y \mathrm{~d} z
$$

where the triple integral denotes integration over an incremental volume of space in a rectangular cartesian co-ordinate system, and the integrand is composed of the squares of the velocities expressed in the same co-ordinate system. Lamb presents the kinetic energy in a useful expression which can be written as 


$$
\begin{gathered}
\mathrm{T}=\rho \iiint\left\{u\left(y \omega_{z}-z \omega_{y}\right)+v\left(z \omega_{x}-x \omega_{z}\right)\right. \\
\left.+w\left(x \omega_{y}-y \omega_{x}\right)\right\} \mathrm{d} x \mathrm{~d} y \mathrm{~d} z
\end{gathered}
$$

where $\omega_{x}, \omega_{y}$, and $\omega_{z}$ are the three components of vorticity in a rectangular Cartesian co-ordinate system. Equation $(\mathrm{J}-2)$ can be shown to reduce to the form of Equation $(\mathrm{J}-1)$. When Equation $(\mathrm{J}-2)$ is transformed to a system of cylindrical co-ordinates and when the condition of axisymmetry is applied, the following equation results:

$$
T=2 \pi \rho \iint\left(r v_{z}-z v_{r}\right) \omega r d r d z
$$

Here, integration in the azimuthal direction has already been performed. The character $\omega$ has the meaning it has used throughout this study, name Iy

$$
\omega=\frac{\partial v_{r}}{\partial z}-\frac{\partial v_{z}}{\partial r}
$$

The integration of Equation (J-3) may be replaced by a summation over all the vortex filaments composing the ring, and this change yields the expression

$$
T=2 \pi \rho \sum\left[K r\left(r v_{z}-z v_{r}\right)\right]
$$

where $k$ denotes the strength of an elementary vortex filament 


$$
\kappa=\omega \delta z \delta r
$$

A similarly useful expression for the impulse of the system of vortex filaments is given by Lamb as

$$
\mathrm{PP}=\pi \rho \iint \mathrm{r}^{2} \omega \mathrm{dr} \mathrm{d} z=\sum \pi \rho\left[k \mathrm{r}^{2}\right]
$$

Again, the integration is to be carried out over an infinite volume expressed in cylindrical co-ordinates, and the summation is over all the vortex filaments of the ring.

To facilitate the development of the expression for kinetic energy, the orientation of a general vortex filament in the circular cross section of the ring is given in Figure J-2. Also shown are the velocities of the filament and the location of the element in the polar co-ordinate system of the core. From the figure it can be seen that

$$
\begin{gathered}
v_{z}=v_{z 0}+s \Omega \cos \theta \\
v_{r}=s \Omega \sin \theta
\end{gathered}
$$

where $\mathrm{v}_{\mathrm{z} 0}$ is the translational velocity of the ring, $\mathrm{s}$ is the distance between the center of the ring cross section and a particular filament, and $\Omega$ is the angular velocity of the core. Substituting these expressions into Equation $(\mathrm{J}-4)$ and expanding gives 


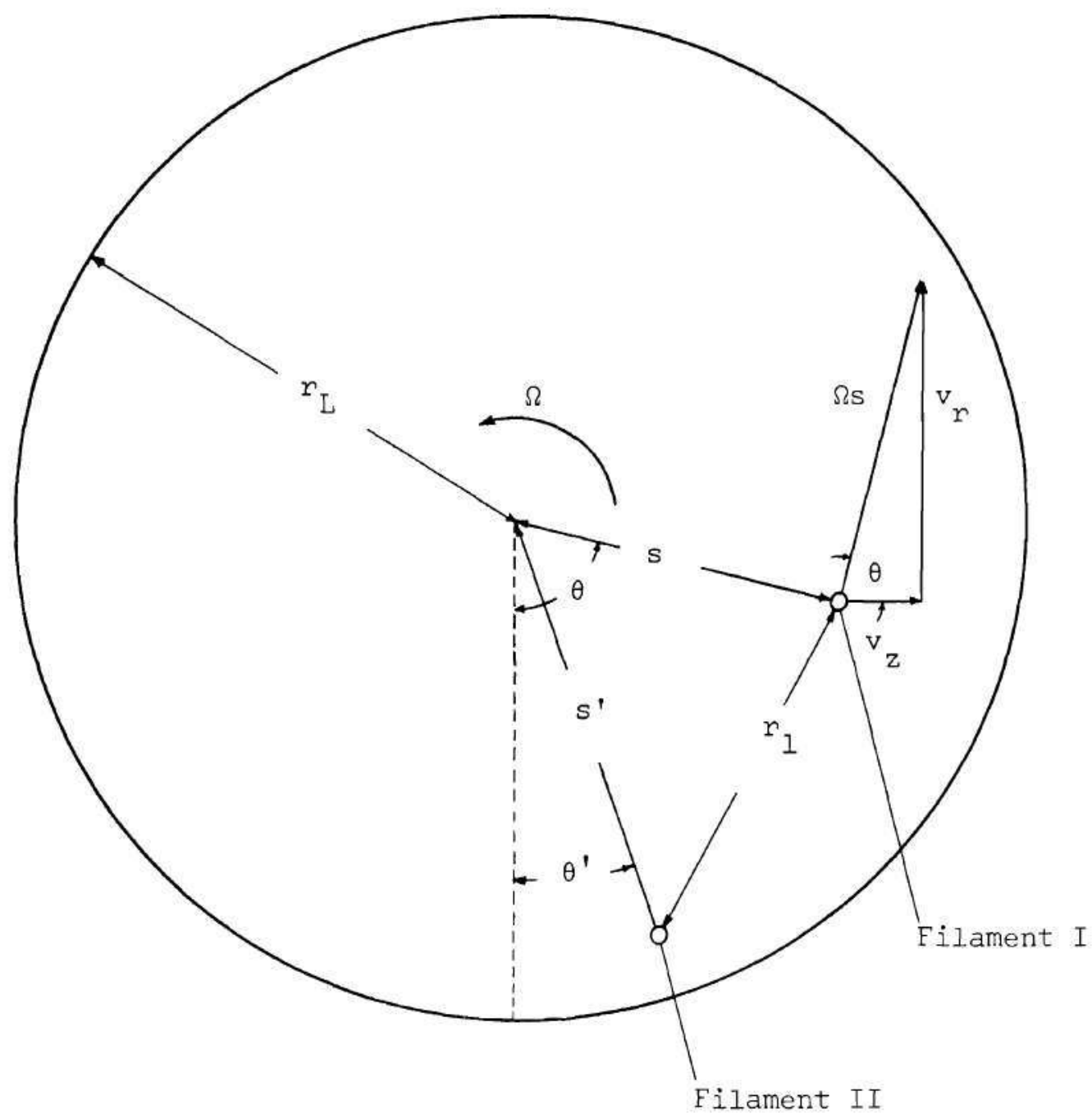

\begin{tabular}{|l|c|c|}
\hline \multicolumn{2}{|c|}{ LOCATION OF VORTEX FILAMENTS } \\
\hline \hline & $\begin{array}{l}\text { Cylindrical } \\
\text { Co-ordinates }\end{array}$ & $\begin{array}{c}\text { Spherical } \\
\text { Co-ordinates }\end{array}$ \\
\hline Filament I & $z, r$ & $s^{\prime}, \theta$ \\
Filament II & $z^{\prime}, r^{\prime}$ & $s^{\prime}, \theta^{\prime}$ \\
\hline
\end{tabular}

Figure J-2. Orientation of Vortex Filaments in Vortex Core 


$$
\frac{T}{2 \pi \rho}=\Sigma \kappa r^{2} v_{z 0}+\Sigma k r^{2}(s \Omega \cos \theta)
$$

$$
-\Sigma \kappa r z(s \Omega \sin \theta)
$$

It is specified that the impulse of the solid, rotating core be equal to the impulse of the system of vortex filaments, in which case Equation ( J-5) implies that

$$
\Sigma \kappa r^{2} v_{z 0}=\Sigma \kappa r_{0}^{2} v_{z_{0}}
$$

and this in turn gives

$$
\begin{gathered}
\frac{T}{2 \pi \rho}=\sum k r_{0}^{2} v_{z 0}+\sum k r^{2}(s \Omega \cos \theta) \\
-\sum k r_{z}(s \Omega \sin \theta)
\end{gathered}
$$

where $r_{0}$ is the radius of the vortex ring. From the geometry of Figure $\mathrm{J}-2$ it may also be seen that

$$
\begin{aligned}
& r=r_{0}-s \cos \theta \\
& z=z_{0}+s \sin \theta
\end{aligned}
$$

Substituting these values into Equation ( J-8) gives 


$$
\begin{aligned}
\frac{T}{2 \pi \rho} & =\Sigma k r_{0}^{2} v_{z 0}+\sum\left\{k\left(r_{0}-s \cos \theta\right)^{2} s \Omega \cos \theta\right. \\
& \left.-k\left(r_{0}-s \cos \theta\right)\left(z_{0}+s \sin \theta\right) s \Omega \sin \theta\right\}
\end{aligned}
$$

Now, the summation of Equation $(\mathrm{J}-9)$ is replaced by an integration in polar co-ordinates over the body, i.e. for $0 \leq s \leq r_{L}$ and $0 \leq \theta \leq 2 \pi$. Hence,

$$
\begin{gathered}
\frac{T}{2 \pi \rho}=\int_{0}^{r_{L}} \int_{0}^{2 \pi} \omega r_{0}^{2} v_{z 0} s d s d \theta+\int_{0}^{r_{L}} \int_{0}^{2 \pi} \omega \Omega\left\{r_{0}^{2} s \cos \theta\right. \\
-2 r_{0} s^{2} \cos ^{2} \theta+s^{3} \cos ^{3} \theta-r_{0} z_{0} s \sin \theta+z_{0} s^{2} \sin \theta \cos \theta \\
\left.-r_{0} s^{2} \sin ^{2} \theta+s^{3} \sin ^{2} \theta \cos \theta\right\} s d s d \theta
\end{gathered}
$$

Since all the vortex filaments are of equal strength, the vorticity may be removed from under the integral sign. Integration of Equation ( $J-10)$ with respect to $\theta$ gives

$$
\frac{T}{2 \pi \rho}=\int_{0}^{r} L r_{0}^{2} v_{z 0} \omega 2 \pi s d s+\int_{0}^{r_{L}} \omega \frac{\omega}{2}\left\{-2 r_{0} s^{2} \pi-r_{0} s^{2} \pi\right\} s d s
$$

Here, the equality

$$
\Omega=\frac{\omega}{2}
$$


has been applied. Finally, integration of Equation (J-ll) with respect to $s$ gives

$$
\frac{T}{2 \pi \rho}=r_{0}^{2} v_{z 0}\left(\omega \pi r_{L}^{2}\right)-\frac{3}{8} \frac{r_{0}}{\pi}\left(\omega \pi r_{L}^{2}\right)^{2}
$$

which in turn simplifies to

$$
\frac{T}{2 \pi \rho}=r_{0}^{2} v_{z 0} \Gamma-\frac{3}{8} \frac{r_{0} \Gamma^{2}}{\pi}
$$

with the knowledge that for solid body rotation

$$
\Gamma=\omega\left(\pi r_{L}^{2}\right)
$$

where $\Gamma$ denotes the circulation of the vortex ring. Equation ( $J-12$ ) gives a simple relation between the velocity, radius, circulation, and the energy per unit mass of the ring. To determine the velocity of the vortex ring, the next task is to evaluate the kinetic energy of the rotating core.

In a system of cylindrical co-ordinates for which axisymmetry exists, the kinetic energy of the ring is given by

$$
T=\pi \rho \iint\left(v_{z}^{2}+v_{r}^{2}\right) r d r d z
$$

Introducing the stream function defined by 


$$
v_{z}=\frac{1}{r} \frac{\partial \psi}{\partial r} \quad \text { and } \quad v_{r}=-\frac{1}{r} \frac{\partial \psi}{\partial z}
$$

into Equation (J-13) and simplifying gives

$$
T=\pi \rho \iint\left(v_{z} \frac{\partial \psi}{\partial r}-v_{r} \frac{\partial \psi}{\partial z}\right) d r d z
$$

Expanding this relation to the form

$$
\frac{T}{\pi \rho}=\int\left\{\int v_{z} \frac{\partial \psi}{\partial r} d r\right\} d z-\int\left\{\int v_{r} \frac{\partial \psi}{\partial z} d z\right\} d r
$$

integrating by parts inside the brackets, and simplifying by applying the definition of vorticity, i.e.

$$
\omega=-\frac{1}{r} \frac{\partial^{2} \psi}{\partial z^{2}}+\frac{1}{r} \frac{\partial \psi}{\partial z}-\frac{1}{r^{2}} \frac{\partial^{2} \psi}{\partial r^{2}}
$$

produces the relation

$$
T=\pi \rho \iint \psi \omega d z d r
$$

The kinetic energy of the ring is given in terms of the stream function and vorticity distributions within the ring. As in the case of previous relations, the integration is to be performed over the entire $z, r$ plane, but since $\omega=0$ outside the ring, the integration is necessary only over the core of the ring.

Now, the stream function distribution with in the ring, the 
rotating solid body, must be found. A standard development employing the concept of mass flux through a circular region is derived by both Lamb and Ringleb [9]. The stream function at the point $(z, r)$ due to a single-filament vortex located at the point $\left(z^{\prime}, r^{\prime}\right)$ is given by the equation

$$
\psi(z, r)=\frac{\omega}{2 \pi}\left(r r^{\prime}\right)^{\frac{1}{2}}\left\{\left(\frac{2}{X}-X\right) E_{I}(X)-\frac{2}{X} E_{I}(X)\right\}
$$

provided that

$$
x^{2}=1-\frac{r_{1}^{2}}{r_{2}^{2}}=\frac{4 r r^{\prime}}{\left(z-z^{\prime}\right)^{2}+\left(r+r^{\prime}\right)^{2}}
$$

Here $F_{1}(X)$ and $E_{1}(X)$ are the complete elliptic integrals of the first and second kind, and $r_{1}$ and $r_{2}$ are the smallest and greatest distances, respectively, between the point $(z, r)$ and the vortex filament. Cayley [42] presents Landen's transformation which allows a reduction of Equation $(J-15)$ to the form

$$
\psi=\frac{\omega}{2 \pi}\left(r_{1}+r_{2}\right)\left\{F_{1}\left(X^{\prime}\right)-E_{1}\left(X^{\prime}\right)\right\}
$$

where

$$
X^{\prime}=\frac{r_{2}-r_{1}}{r_{2}+r_{1}}
$$

For the case of the solid core composed of a number of vortex filaments, 
this expression must be integrated over all the filaments. Using cylindrical co-ordinates once again and integrating gives

$$
\psi=\frac{1}{2 \pi} \iint\left\{E_{1}\left(\frac{r_{2}-r_{1}}{r_{2}+r_{1}}\right)-E_{1}\left(\frac{r_{2}-r_{1}}{r_{2}+r_{1}}\right)\right\}\left(r_{1}+r_{2}\right) \omega^{\prime} d r^{\prime} d z^{\prime}
$$

This is the stream function at the point $(z, r)$ due to a distribution of vortex filaments of strength $\omega^{\prime}$ located at the general points $\left(z^{\prime}, r^{\prime}\right)$. Since the vorticities of all filaments are equal and since no filaments exist outside the solid core, the integration need be performed only over the core. Consequently the parameter $r_{1}$ is very small compared to $r_{2}$.

Cayley presents series approximations to the elliptic integrals of Equation $(J-17)$. For small values of $r_{1}$, these functions simplify to the expression

$$
F_{1}\left(\frac{r_{2}-r_{1}}{r_{2}+r_{1}}\right)=\frac{1}{2} \ln \frac{16\left(r_{1}+r_{2}\right)^{2}}{4 r_{1} r_{2}}
$$

and

$$
E_{1}\left(\frac{r_{2}-r_{1}}{r_{2}+r_{1}}\right)=1
$$

Since $r_{1} \ll r_{2}$ and $r_{2} \cong 2 r_{0}$, a further reduction is possible; hence, the stream function simplifies to

$$
\psi=\frac{1}{\pi} \iint\left\{\frac{1}{2} \ln \left(\frac{8 r_{0}}{r_{1}}\right)-1\right\}_{r_{0}} \omega r^{\prime} d r^{\prime} d z^{\prime}
$$


Since $\omega$ is zero outside the solid core and constant inside, the integration may be performed using polar co-ordinates over the cross section to give

$$
\psi(s, \theta)=\frac{\omega}{2 \pi} r_{0} \int_{0}^{r_{L}} \int_{0}^{2 \pi}\left\{\ln \left(\frac{8 r_{0}}{r_{I}}\right)-2\right\} s^{\prime} d s^{\prime} d \theta^{\prime}
$$

In the new co-ordinate system, the parameter $r_{1}$ is the distance between the location of the vortex element, $\left(s^{\prime}, \theta^{\prime}\right)$, and the point where the stream function is being computed, $(s, \theta)$. Hence, $r_{1}$ is given by the Law of Cosines as

$$
r_{1}=\left\{s^{2}+s^{\prime 2}-2 s s^{\prime} \cos \left(\theta-\theta^{\prime}\right)\right\}^{\frac{1}{2}}
$$

which can be seen in Figure J-2. Integration of Equation (J-19) involves isolation of the term $\ln \left(r_{1}\right)$ and integration of it with respect to $\theta^{\prime}$. Accordingly

$$
\int_{0}^{2 \pi} \ln \left\{s^{2}+s^{\prime 2}-2 s s^{\prime} \cos \left(\theta-\theta^{\prime}\right)\right\} d \theta= \begin{cases}2 \pi \ln s^{\prime} & \text { if } s^{\prime}>s \\ 2 \pi \text { In } s & \text { if } s^{\prime}<s\end{cases}
$$

Incorporating this fact into Equation $(\mathrm{J}-19)$ and judiciously regathering the terms resulting from the integration gives

$$
\psi=-\frac{\omega r_{0}}{2 \pi}\left\{\int_{0}^{s}\left\langle 2 \pi\left[\ln \left(8 r_{0}\right)-2\right]+2 \pi \ln s\right\rangle s^{\prime} d s^{\prime}\right.
$$




$$
\left.+\int_{s}^{r_{L}}\left\langle 2 \pi\left[\ln \left(8 r_{0}\right)-2\right]+2 \pi \ln s^{\prime}\right\rangle s^{\prime} d s^{\prime}\right\}
$$

Performing this integration over $s^{\prime}$ and simplifying gives finally,

$$
\psi(s, \theta)=-\frac{\omega r_{0} r_{L}^{2}}{2}\left\{\ln \left(\frac{8 r_{0}}{r_{L}}\right)-\frac{3}{2}-\frac{1}{2} \frac{s^{2}}{r_{L}^{2}}\right\}
$$

Equation (J-20) gives at a point in the core of the ring the stream function which is due to a distribution of equal-strength vortex filaments. Integration of this function throughout the core allows evaluation of the kinetic energy of the ring which is given by Equation ( $\mathrm{J}-14$ ). This in turn may be substituted into Equation ( $\mathrm{J}-\mathrm{I2}$ ) to give the vortex velocity.

Combining Equations $(J-20)$ and $(J-14)$ and expanding the result gives

$$
\frac{T}{2 \pi \rho}=-\frac{1}{2} \omega \int_{0}^{r_{L}} \int_{0}^{2 \pi}\left\{-\frac{\omega r_{0} r_{L}^{2}}{2} \ln \left(\frac{8 r_{0}}{r_{L}}\right)+\frac{3}{4} \omega r_{0} r_{L}^{2}\right\} s d s d \theta
$$

$$
-\frac{1}{2} \omega \int_{0}^{r_{L}} \int_{0}^{2 \pi}\left\{\frac{1}{4} \omega r_{0} s^{2}\right\} s d s d \theta
$$

Integrating, gathering terms, simplifying, and employing the equality for solid body rotation

$$
\Gamma=\omega \pi r_{L}^{2}
$$


yields

$$
\frac{T}{2 \pi \rho}=\frac{r_{0} \Gamma^{2}}{4 \pi}\left\{\ln \left(\frac{8 r_{0}}{r_{L}}\right)-\frac{7}{4}\right\}
$$

Here, the kinetic energy of the vortex ring is given simply in terms of the strength and geometry of the ring. Substituting Equation (J-22) into Equation ( $\mathrm{J}-12)$ and simplifying gives finally

$$
v_{z 0}=\frac{\Gamma}{4 \pi r_{0}}\left\{\ln \left(\frac{8 r_{0}}{r_{L}}\right)-\frac{1}{4}\right\}
$$

This is the well-known result of Lamb which gives the velocity of a vortex ring in terms of its strength and geometry.

To understand the limitations on Equation (J-23) as an accurate description of a physically reasonable phenomenon, the assumptions employed in its derivation are repeated here:

1. The ring is a torus of circular cross section which rotates as a solid body. The flow field outside the ring is irrotational.

2. The radius of the core is very small compared to the radius of the ring.

Although Equation ( $\mathrm{J}-23$ ) appears to be a neatly packaged answer to a comparatively complex problem, a difficulty exists. The radius of the core is unknown. According to Ringleb, this fact has led Sommerfeld [43] and others to express the opinion that these results are useless because of the unknown radius $r_{L}$ and the arbitrary assumption that the circulation is constant for the circular vortex lines within the ring. 
It is the purpose of this appendix to develop the expression for the translational velocity of fluid which is a great distance removed from an axisymmetric spherical vortex. The analysis is one of perfect fluid theory. Consequently, the viscosity of the fluid does not enter into this derivation, and there is no mechanism for the decay of the circulation of the sphere. The physical system corresponds to the axisymmetric analogy of a pair of standing vortices in an invisid fluid of uniform velocity.

When the definition of vorticity

$$
\omega=\frac{\partial v_{r}}{\partial z}-\frac{\partial v_{z}}{\partial r}
$$

is written in terms of the stream function, the resulting equation is given by

$$
\omega=-\frac{1}{r} \frac{\partial^{2} \psi}{\partial z^{2}}+\frac{1}{r^{2}} \frac{\partial \psi}{\partial r}-\frac{1}{r} \frac{\partial^{2} \psi}{\partial r^{2}}
$$

Hill [44] solves this equation by assuming that the vorticity can be expressed by

$$
\omega=r F(\psi)
$$


where $F(\psi)$ is a constant given by

$$
F(\psi)=-5 \bar{A}
$$

Combining these relations gives

$$
\frac{\partial^{2} \psi}{\partial z^{2}}-\frac{1}{r} \frac{\partial \psi}{\partial r}+\frac{\partial^{2} \psi}{\partial r^{2}}=5 \bar{A} r^{2}
$$

which has the solution

$$
\psi=\frac{\bar{A}}{2} r^{2}\left(a_{0}^{2}-z^{2}-r^{2}\right)
$$

That this is true may be verified by the substitution of Equation $(K-4)$ into Equation $(\mathrm{K}-3)$. Since the stream function vanishes on the sphere

$$
r^{2}+z^{2}=a_{0}^{2}
$$

the stream function distribution of Equation $(K-4)$ represents the flow field inside a sphere of radius $a_{0}$. The geometry of this sphere and its orientation in the $z, r$ plane is given in Figure $k-1$. Combining Equations $(\mathrm{K}-1)$ and $(\mathrm{K}-2)$ gives the vorticity distribution inside the sphere as

$$
\omega=-5 \bar{A} \mathrm{r}
$$




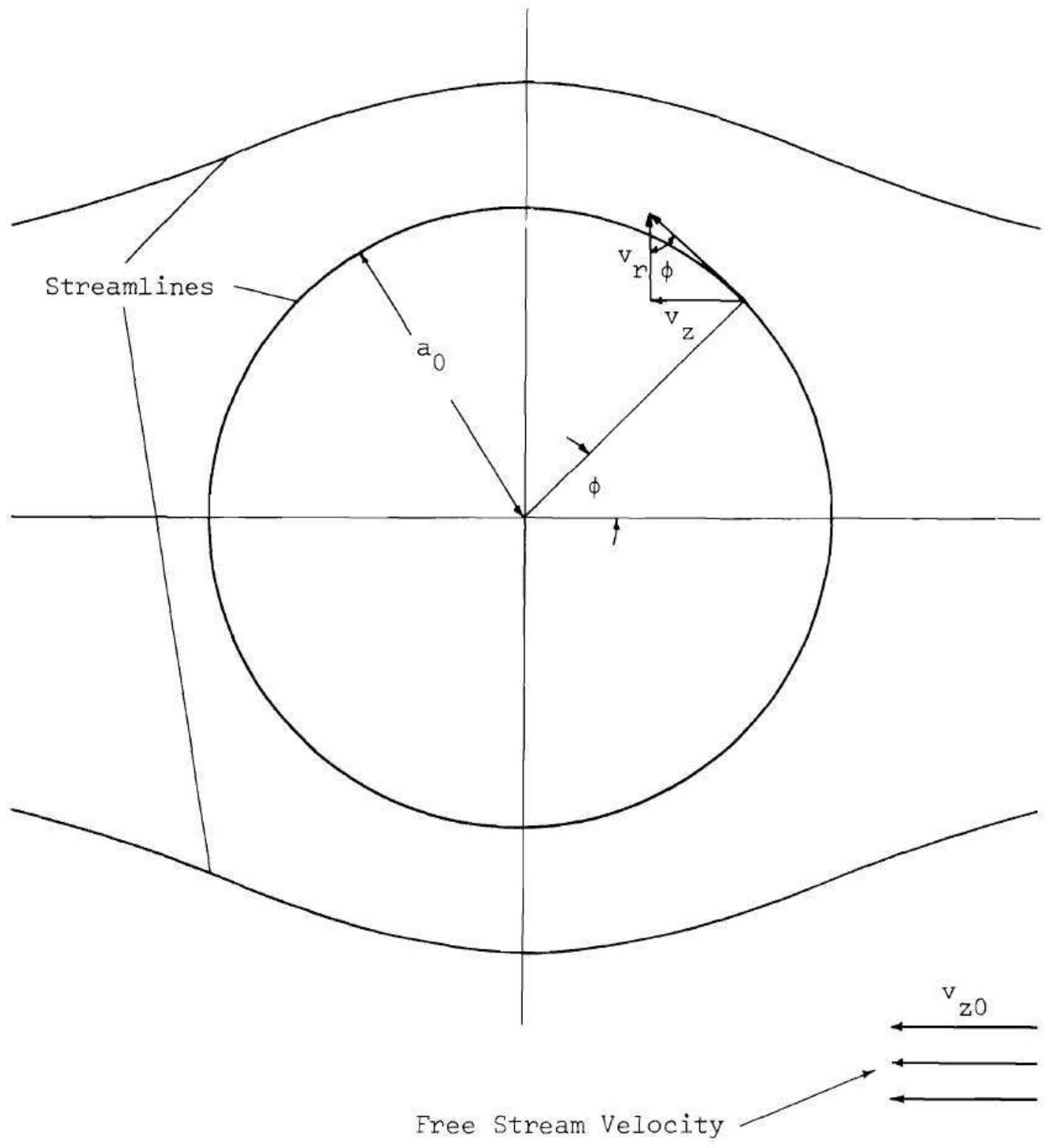

Figure K-l. Orientation of Hill's Spherical Vortex 
If the fluid outside the sphere is moving in the negative $z-$ direction with a velocity $v_{z 0}$, then from perfect fluid theory its stream function distribution is known to be

$$
\psi=\frac{1}{2} v_{z 0} r^{2}\left\{1-\frac{a_{0}^{3}}{\left(z^{2}+r^{2}\right)^{3 / 2}}\right\}
$$

Here again the stream function vanishes on the sphere of radius $a_{0}$. Equations $(\mathrm{K}-4)$ and $(\mathrm{K}-6)$ represent a continuous stream function throughout the field, and since the stream function vanishes on the sphere, the sphere is a streamline of the flow. By definition the velocity normal to the sphere is zero. However, another condition which must be satisfied by the inviscid fluid is that the fluid velocity must be continuous throughout the flow field. Hence, the velocity calculated from the stream function inside the sphere must equal the velocity calculated from the stream function outside the sphere on the sphere. Using the polar co-ordinate system of Figure $\mathrm{K}-1$, this condition may be expressed mathematically by the equation

$$
\mathrm{v}_{\mathrm{zI}}=\mathrm{v}_{\mathrm{zE}}
$$

where the subscripts I and E differentiate between the velocities internal and external to the sphere. Since the axial velocity is given by

$$
\mathrm{v}_{\mathrm{z}}=\frac{1}{r} \frac{\partial \psi}{\partial r}
$$


Equation (K-7) reduces to

$$
\left.\left.\frac{\partial \psi}{\partial r}\right)_{I}=\frac{\partial \psi}{\partial r}\right)_{E}
$$

Differentiating Equations $(K-4)$ and $(K-6)$, evaluating the derivatives on the sphere for which $a_{0}^{2}=r^{2}+z^{2}$, and substituting the results into Equation $(K-8)$ reveals that

$$
\bar{A}=-\frac{3}{2} \frac{v_{z 0}}{a_{0}^{2}}
$$

Combining this fact with Equation $(K-5)$ gives the vorticity distribution inside the sphere as

$$
\omega=\frac{15}{2} v_{z 0} \frac{r}{a_{0}^{2}}
$$

By integrating this vorticity distribution over the semicircle of radius $a_{0}$, the circulation of a section of half the spherical vortex can be computed. Hence

$$
\Gamma=\int_{0}^{a_{0}} \int_{\substack{a^{2}-r^{2} \\ 0}}^{\sqrt{a^{2}-r^{2}}} \frac{15}{2} \frac{v_{0}}{a_{0}^{2}} r d r d z
$$

which is evaluated as

$$
\Gamma=5 \mathrm{v}_{\mathrm{z} 0} \mathrm{a}_{0}
$$


If the size and circulation about half of the spherical vortex are known, then the velocity of the external flow far from the sphere may be calculated as

$$
v_{z 0}=\frac{1}{5} \frac{\Gamma}{a_{0}}
$$

This is the desired result. 
1. Helmholtz, H. U., Uber Intergrale der hydrodynamischen Gleichungen, welche den Wirbelbewegungen entsprechen, Crelle, IV, 1858, Wiss. Abh. I, 101 .

2. Stokes, G. G., "On the Effect of Internal Friction of Fluids on the Motion of Pendulums," Scientific Papers, 3, 55, University Press, Cambridge, 1901.

3. Stokes, G. G., "On the Theories of the Internal Friction of Fluids in Motion," Scientific Papers, 1, 80, University Press, Cambridge, 1901 .

4. Reynolds, 0., "On the Resistance Encountered by Vortex Rings," British Assn. Rep., 1876, Nature, XIV, 477.

5. Thomson, J. J., and Tait, P. G., "Ring Vortex," Natural Philosophy, Art. 546.

6. Thomson, Sir W., "On Vortex Motion," Papers, IV, 13, 1869.

7. Lamb, H., Hydrodynamics (Sixth Edition), Dover Publications, Inc., New York, 1945.

8. Basset, A. B., A Treatise on Hydrodynamics, Vol. II, Dover Publications, Inc., New York, 1961.

9. Ringleb, F. O., Vortex Motion, Research Report 47, Aerophysics Dept., Mississippi State University, 1963.

10. Kelvin, Lord (Thomson, Sir W.), "On Vortex Atoms," PhiZosophical Magazine (4), XXXIV, 20, 1867.

11. Kutta, W. M., "Uber eine mit den Grundlagen des Flugproblems in Beziehung stehende zweidimensionale Strömung," Munchener Berichte, 1910, pp. 1-58.

12. Joukowski, N. E., "Uber die Profile von Trugflügeln," Zeitschrift für Flugtechnik und Motorluftschifahrt, Vol. 1, 1910, p. 280 and Vol. 3, 1912, p. 81 .

13. Hall, M. G., "A Theory for the Core of the Leading Edge Vortex," J. Fluid Mech., Vol. 11, pt. 2, Sept., 1961, pp. 209-228. 
14. Donaldson, C. duP., and Sullivan, R. D., "Behavior of Solutions of the Navier-Stokes Equations for a Complete Class of ThreeDimensional Viscous Vortices," Heat Transfer and Fluid Mechanics Institute, 1960, Proceedings, Stanford University Press, pp. 16-30.

15. Rosenzweig, M. L., Lewellen, W. S., and Ross, D. H., "Confined Vortex Flows with Boundary-Layer Interaction," ATAA Joumal, Vol. 2, No. 12, December, 1964, pp. 2127-2134.

16. Lewellen, W. S., "Linearized Vortex Flows," AIAA Journal, Vol. 3, No. 1, January, 1965, pp. 91-96.

17. Payne, R. B., "Numerical Method for Calculating the Starting and Perturbation of a Two-Dimensional Jet at Low Reynolds Number," Aero. Res. Council (London), Rep. and Mem., No. 3047, 1956.

18. Fromm, J., "The Time Dependent Flow of an Incompressible Viscous Fluid," Methods in Computational Physics, Vol. 3, Academic Press, New York and London, 1964.

19. Harlow, F. H., Welch, J. P., and Shannon, J. E., "Liquid Waves by Computer," Science, Vol. 149, p. 1092.

20. Greenspan, D., Jain, P. C., Manohar, R., Noble, B., and Sakurai, A., "Numerical Studies of the Navier-Stokes Equations," MRC Technical Summary Report No. 482, Math. Research Center, Univ. of Wisc., May, 1964.

21. Pearson, C. E., "A Computational Method for Viscous Flow Problems," J. Fluid Mech., Vol. 21, 1965, p. 611.

22. Esch, R. E., An Alternative Method of Handling Boundary Conditions, and Various Computational Experiments in the Numerical Solution of Viscous Flow Problems, Sperry Rand Res. Center, SRRC-RR-64-64, 1964.

23. Brady, W. G., and Crimi, P., Representation of Propeller Wakes by Systems of Finite Core Vortices, Cornell Aeronautical Laboratory Report No. BB-1665-S-2, 1965.

24. Crimi, P., "Prediction of Rotor Wake Flows," CAL/USSAVLABS Symposium Proceedings, Aerodynamic Problems Associated with V/STOL Aircraft, Vol. I, June, 1966.

25. Du Waldt, F. A., Wakes of Lifting Propellers (Rotors) in Ground Effect, Cornell Aeronautical Laboratory Report No. BB-1665-S-3, 1966.

26. Adams, G. N., "Propeller Research at Canadair Limited," CAL/ USSAVLABS Symposium Proceedings, Aerodynamic Problems Associated with V/STOL Aircraft, Vol. I, June, 1966. 
27. Gray, R. B., On the Motion of the Helical Vortex Shed from a Single-Bladed Hovering Model Helicopter Rotor and Its Application to the Calculation of the Spanwise Aerodynamic Loading, Princeton Univ. Aero. Eng. Dept., Report No. 313, 1955.

28. Simons, I. A., Blade/Vortex Interaction on Helicopter Rotors in Forward Flight, Univ. of Southampton, Inst. of Sound and Vibrations, Rep. 127, 1965.

29. Banerji, S. K., and Barave, R. V., "On Oberbeck's Vortices," Phil. Mag., S. 7, Vol. 11, No. 73, May, 1931, pp. 1057, 1081.

30. Okabe, J., and Inoue, S., "The Generation of Vortex Rings," Kyushu Univ., Rep. Res. Inst. Appl. Mech., Vol. 8, No. 32, 1960, pp. 91-101.

31. Margavey, R. H., and MacLatchy, C. S., "The Formation and Structure of Vortex Rings, I," Canadian Journal of Physics, Vol. 42, Apr., 1964, pp. 678-683.

32. Pearson, C. E., A Computational Method for Time-Dependent TwoDimensional Incompressible Viscous Flow Problems, Sperry Rand Research Center, SRRC-RR-64-17, 1964.

33. O'Brien, G. G., Hyman, M. A., and Kaplan, S., "A Study of the Numerical Solution of Partial Differential Equations," Joumal of Math. and Phys., Vol. 29, 1951, pp. 223-25l.

34. Hildebrand, F. B., Methods of Applied Mathematics, Prentice-Hall, Inc., Englewood Cliffs, N. J., 1961, p. 345.

35. Kurzrock, J. W., and Mates, R. E., Exact Numerical Solutions of the Time-Dependent Compressible Navier-Stokes Equations, AIAA Paper No. 66-30, AIAA Third Aerospace Sciences Meeting, New York, New York, Jan. 24-26, 1966.

36. Pai, S. I., Fluid Dynamics of Jets, D. Van Nostrand Company, Inc., Princeton, N. J., 1954.

37. Akmetov, D. G., and Kisarov, O. P., "Gyrodynamic Structure of a Vortex Ring," PMTF-Zhurnal Prikladnoi Mekhaniki i Tekhnicheskoi Fiziki, July-Aug., 1966, pp. 120-123.

38. DuFort, E. C., and Frankl, S. P., Math. Tables and other Aids to Computation, Vol. ?, p. 135.

39. Faddeeva, V. N., Computational Methods of Linear Algebra, Dover Publications, Inc., New York, 1959. 
40. Carré, B. A., "The Determination of the Optimum Accelerating Factor for Successive Over-relaxation," Computer Joumal, Vol. 4, No. $1,1961, \mathrm{pp} .73-78$.

41. Peaceman, D. W., and Rachford, H. H., Jr., "The Numerical Solution of Parabolic and Elliptic Differential Equations," J. Soc. Indust. Appl. Math., Vol. 3, No. 1, March, 1965, pp. 28-41.

42. Cayley, A., An Elementary Treatise on Elliptic Functions, Dover Publications, New York, 1961.

43. Sommerfeld, A., "Theorie der Wirbel," Mechanik der derformierbaren Medien, Kap. IV, Liepzig, 1945.

44. Hill, M. J. M., "On the Motion of Fluid, Part of Which is Moving Rotationally and Part Irrotationally," Philosophical Transactions, Vol. CLXXV, Pt. II, 1884, pp. 363-409. 
VITA

Kenton David Whitehead was born in Chicago, Illinois on September 16, 1939. His parents are Lawrence E. and Helen F. Whitehead. He attended the elementary schools of Lombard, Illinois and in June, 1957 was graduated from Glenbard Township High School in Glen Ellyn, Illinois.

In September, $1957 \mathrm{Mr}$. Whitehead entered Purdue University, and in June, 1961 he was awarded the degree of Bachelor of Science in Aeronautical Engineering. He continued his education as a graduate student at the Georgia Institute of Technology from which he received the degree of Master of Science in Aeronautical Engineering in June, 1963. An NDEA Fellowship allowed him to continue under the doctoral program. Mr. Whitehead has been employed as an engineer for several summers by the Allegany Ballistics Laboratory of Cumberland, Maryland, the Marshall Space Flight Center of Huntsville, Alabama, and the Lockheed-Georgia Company of Marietta, Georgia. He has also been employed part time in various capacities as a graduate research assistant and a graduate teaching assistant by the Georgia Institute of Technology. Mr. Whitehead is a member of Phi Eta Sigma, Sigma Gamma Tau, Tau Beta Pi, and Sigma $\mathrm{Xi}$.

On September 18, 1964 he was married to Mary Elizabeth Johnson, daughter of Mr. and Mrs. James M. Johnson of Miami, Florida. 